\title{
Novel genetic causes and pathological mechanisms of neurological and mitochondrial disorders
}

Citation for published version (APA):

Nguyen, M. H. T. (2017). Novel genetic causes and pathological mechanisms of neurological and mitochondrial disorders. [Doctoral Thesis, Maastricht University]. Datawyse / Universitaire Pers Maastricht. https://doi.org/10.26481/dis.20171218mhtn

Document status and date:

Published: 01/01/2017

DOI:

10.26481/dis.20171218mhtn

Document Version:

Publisher's PDF, also known as Version of record

\section{Please check the document version of this publication:}

- A submitted manuscript is the version of the article upon submission and before peer-review. There can be important differences between the submitted version and the official published version of record.

People interested in the research are advised to contact the author for the final version of the publication, or visit the DOI to the publisher's website.

- The final author version and the galley proof are versions of the publication after peer review.

- The final published version features the final layout of the paper including the volume, issue and page numbers.

Link to publication

\footnotetext{
General rights rights.

- You may freely distribute the URL identifying the publication in the public portal. please follow below link for the End User Agreement:

www.umlib.nl/taverne-license

Take down policy

If you believe that this document breaches copyright please contact us at:

repository@maastrichtuniversity.nl

providing details and we will investigate your claim.
}

Copyright and moral rights for the publications made accessible in the public portal are retained by the authors and/or other copyright owners and it is a condition of accessing publications that users recognise and abide by the legal requirements associated with these

- Users may download and print one copy of any publication from the public portal for the purpose of private study or research.

- You may not further distribute the material or use it for any profit-making activity or commercial gain

If the publication is distributed under the terms of Article $25 \mathrm{fa}$ of the Dutch Copyright Act, indicated by the "Taverne" license above, 


\section{Novel Genetic Causes and Pathological Mechanisms of Neurological and Mitochondrial Disorders}

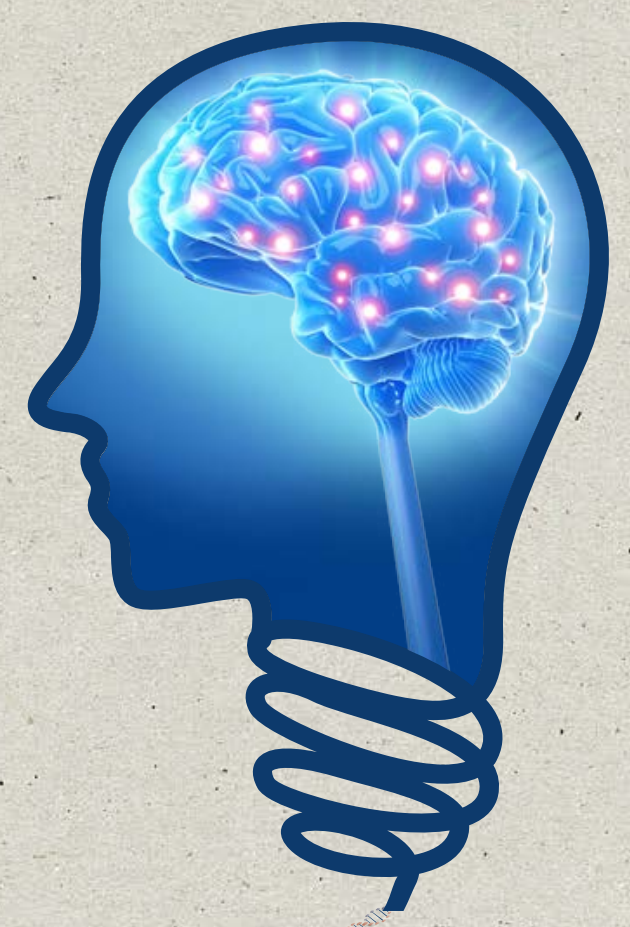


(C) Minh Nguyen, Maastricht 2017

No parts of this thesis may be reproduced or transmitted in any form or by any means, without prior permission in writing by the author, or when appropriate, by the publishers of the publications.

Layout and printing: Datawyse/ Universitaire Pers Maastricht Cover: Quynh Doan and Ubbo Noordhof

ISBN: 9789461597861 


\title{
Novel genetic causes and pathological mechanisms of neurological and mitochondrial disorders
}

\author{
PROEFSCHRIFT
}

Ter verkrijging van de graad van doctor aan de Universiteit Maastricht, op gezag van de Rector Magnificus, Prof. Dr. Rianne M. Letschert volgens het besluit van het College van Decanen, in het openbaar te verdedigen op maandag 18 december 2017 om 13:30 uur.

door

Minh Hoang Tuyet Nguyen

Geboren op 29 december 1978 te Hanoi Vietnam. 


\section{Promotor}

Prof. Dr. H.J.M. Smeets

\section{Copromotores}

Dr. M. Gerards

Dr. I.F.M. de Coo (Erasmus MC Rotterdam)

\section{Beoordelingscommissie}

Prof. Dr. C.G. Faber (voorzitter)

Prof. Dr. T.M.C.M. de Kok

Prof. Dr. E. Morava-Kozicz (Universitair Ziekenhuis Leuven, BE)

Dr. M. Muller (Liège Université, BE)

Dr. M. van Geel 


\section{Contents}

Abbreviations

Chapter 1 General Introduction and Thesis outline

Chapter 2 Pathogenic CWF19L1 variants as a novel cause of autosomal recessive cerebellar ataxia and atrophy

Chapter 3 Novel pathogenic SLC25A46 splice-site mutation causes an optic atrophy spectrum disorder

Chapter 4 Mutations in COX18 cause isolated complex IV deficiency, associated with neurodegeneration, chorea dystonic movement and type 1 diabetes

Chapter 5 Whole exome sequencing reveals HKDC1 and MED20 as candidate genes in a child with a neurodegenerative disorder and congenital cataract

Chapter 6 Whole exome sequencing is the preferred, first strategy to identify known and novel causes of mitochondrial disease

Chapter 7 General discussion

Summary

Valorisation

Acknowledgements 169

Curriculum Vitae

List of publications 



\title{
Abbreviations
}

\author{
AD Alzheimer's disease \\ $A D \quad$ autosomal dominant \\ ADOA Autosomal Dominant Optic Atrophy \\ ALS Amyotrophic lateral sclerosis \\ AR autosomal recessive \\ ARCA Autosomal recessive cerebellar ataxia \\ CA Cerebellar Ataxia \\ CCS circular consensus sequencing \\ CMT Charcot-Marie Tooth Disease \\ CNS central nervous system \\ CNV copy number variations \\ CPEO Chronic Progressive External Ophthalmoplegia \\ DD differential diagnosis \\ DMEM Dulbecco modified Eagle medium \\ DRP1 Dynamin-related protein 1 \\ $\mathrm{FADH}_{2}$ flavin adenine dinucleotide \\ FD familial dysautonomia \\ HGP Human Genome Project \\ HSP Hereditary Spastic Paraplegia \\ iPSCs induced pluripotent stem cells \\ KSS Kearns-Sayre syndrome \\ LHON Leber's Hereditary Optic Neuropathy \\ MBP myelin basic protein \\ MD mitochondrial disorders \\ MDS mtDNA depletion syndromes \\ MELAS Mitochondrial Myopathy, Encephalopathy, Lactic Acidosis, and Stroke-Like \\ Episodes \\ MERRF myoclonic epilepsy with ragged red fibers \\ MFN mitofusin \\ MIDD maternally inherited diabetes mellitus and deafness \\ MIPs molecular inversion probes \\ MIRAS Mitochondrial-associated Recessive Ataxia Syndrome \\ MNGIE mitochondrial neurogastrointestinal encephalomyopathy \\ MO morpholino \\ MRI magnetic resonance imaging \\ mtDNA mitochondrial DNA \\ $\mathrm{NADH}$ nicotinamide adenine dinucleotide
}


NARP neuropathy, ataxia, retinal pigmentosa

NGS next-generation sequencing

NMD nonsense mediated mRNA decay

OMIM Online Mendelian Inheritance in Man

OXPHOS oxidative phosphorylation

PD Parkinson disease

PEO Progressive External Ophthalmoplegia

POLRMT mitochondrial RNA polymerase

PTC premature termination codon

rRNA ribosomal RNA

SANDO Sensory Ataxia, Neuropathy, Dysarthria, Ophthalmoparesis

SMRT single-molecule real-time sequencing

TPR tandem tetratricopeptide repeat

tRNA transfer RNA

VUS variants of unknown significance

WES whole exome sequencing

WGS whole genome sequencing

$\mathrm{XLR}$ recessive $\mathrm{X}$-linked

ZMW zero-mode waveguide 
Chapter

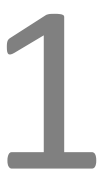

General Introduction and Thesis outline 



\section{INTRODUCTION}

\subsection{Neurological diseases}

Neurological disorders primarily affect and impair the functioning of the brain and/or neurological system. These disorders can be the result of structural, electrical or biochemical abnormalities in the brain or nervous system and are characterized by a broad range of clinical manifestations. Common symptoms include malfunction of the motor system, poor coordination, impaired sensory network, diminished cognitive function and altered levels of consciousness [1]. Hundreds of millions of people around the world suffer from different forms of neurological disorders. For example, worldwide, 50 million people have epilepsy and it is estimated that globally 47.5 million people have dementia, with 7.7 million new cases every year. Neurological disorders can affect people of all ages and have a devastating impact on a person's life, not only leading to major morbidity and mortality, but also affecting community resources and health-care professionals [2].

Neurological disorders can have different causes, like genetic defects, infections, trauma, and brain injury, but they can also develop as a result of an unhealthy environment, for example malnutrition [3]. In this thesis, I will focus on neurological disorders, in which a genetic defect in the mitochondria are expected to play a pathological role. Neurons are highly energy demanding and mitochondrial dysfunction can have both a primary, causative or, secondary, modifying role in the pathogenesis of many neurological disorders. Both primary and secondary mitochondrial neurological diseases are clinically and genetically highly complex.

\subsection{Mitochondrial neurological disease and causes}

Mitochondria are dynamic cellular organelles surrounded by a double membrane, which are crucial for many metabolic processes in the cell. Mitochondria are thought to be derived from a bacterium, which became part of an ancestral eukaryote cell through endosymbiosis. These symbiont cells and their DNA have undergone evolutionary reduction that turned the bacterium into organelles, which although dependent on the host cell, still carry a small amount of genetic material, the mitochondrial DNA (mtDNA) [4]. The most important role for mitochondria is the production of energy in the form of ATP through a process called oxidative phosphorylation or OXPHOS and the term mitochondrial diseases is commonly used for diseases caused by defective OXPHOS. Besides energy production, mitochondria play a pivotal role in the regulation of cellular homeostasis and survival. Metabolite synthesis, calcium signaling, lipid metabolism and apoptosis are only a few of the many cellular processes in which mitochondria play a key role $[5,6]$. 
The importance of mitochondria and mitochondrial processes is illustrated by the impact of mitochondrial dysfunction on cells, organs and organisms (Figure 1).

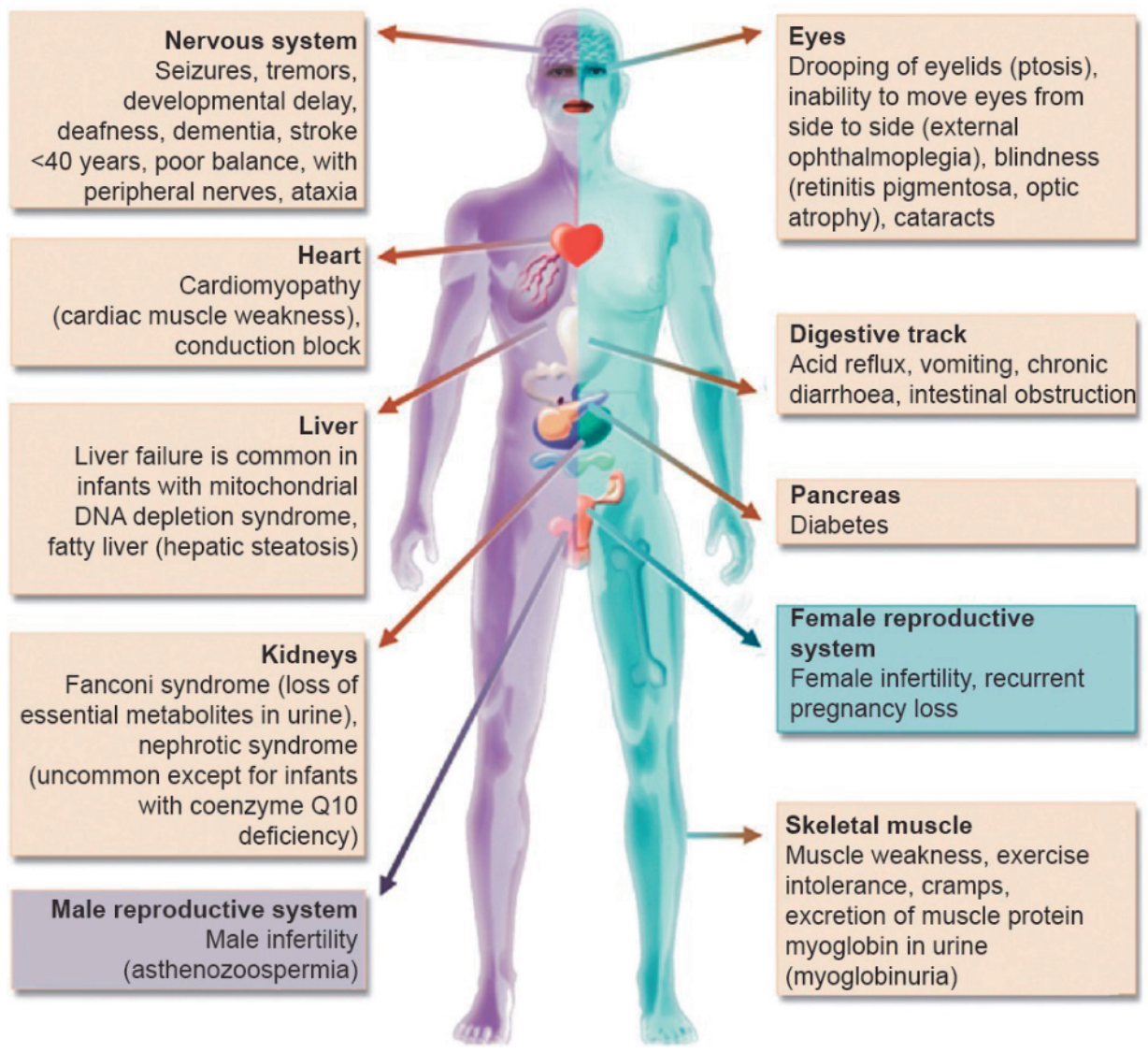

Figure 1: Schematic picture showing the clinical features and the organs affected by mitochondrial disease [7]

Not surprisingly, the tissues with the highest energy demand are most often affected and, therefore, neurological pathology is very common in mitochondrial disease. Genetic mitochondrial diseases can be caused by mutations in the mtDNA or in the nuclear DNA. Mitochondrial diseases caused by mtDNA defects do not follow the rules of Mendel, but are inherited through the maternal line with variable expression. The paternal mitochondria, and with it the paternal mtDNA, is eliminated at fertilization [8]. Nuclear gene mutations segregate in an autosomal dominant, autosomal recessive or X-linked fashion. The exact prevalence of mitochondrial disease is difficult to estimate due to the extensive genetic and clinical heterogeneity. However, the minimum birth prevalence for pediatric mitochondrial disease has been estimated as 6.2/100,000 [9]. This frequency could be higher in communities with a high level of consanguineous marriage, due to the increased risk for autosomal recessive disorders [10]. In adults, mitochondri- 
al disease has a prevalence of $\sim 1$ in 4300 [9], making it one of the most common causes of inherited neurological disorders. mtDNA mutations have been detected in over $75 \%$, of adult patients with overt mitochondrial disease, in which the LHON (Leber's Hereditary Optic Neuropathy) and m.3243A>G MELAS (Mitochondrial Myopathy, Encephalopathy, Lactic Acidosis, and Stroke-Like Episodes) mutations account for $75 \%$ of the mtDNA mutations [11].

The mtDNA differs in many aspects from the nuclear DNA. It is a double- stranded circular, histone-free molecule of $16.6 \mathrm{~kb}$ in size with no introns and a genetic code different from nuclear genes [12]. The structure and gene organization of the mtDNA are highly conserved among mammals [13]. The mtDNA encodes 13 protein subunits required for the OXPHOS enzyme complexes I, III, IV and V, two ribosomal RNAs (rRNA) and 22 transfer RNAs (tRNA). The remainder of the OXPHOS subunits as well as all other proteins essential for the replication, transcription and translation of the mtDNA are encoded by nuclear genes and imported into the mitochondria [14]. An important feature of mitochondria and the mtDNA is that each mitochondrion can contain multiple copies of mtDNA and each cell multiple copies of mitochondria. Adult somatic cells typically contain from a few hundred to several thousand mitochondria with the highest number in the tissues with the highest energy demand (e.g. brain, heart, liver, skeletal muscle). Oocytes contain the highest number of mitochondria $(100,000$ to 600,000 copies in a human oocyte) [15], which declines during development both in germline and somatic cells (genetic bottleneck) and increases again during oogenesis and differentiation [16]. As a consequence of being a multi-copy genome, mtDNA mutations can occur at varying levels. A mtDNA mutation which is present in only a subset of mtDNA molecules is called a heteroplasmic mutation, whereas, a mutation, which is present in all mtDNA molecules is called a homoplasmic mutation. In the presence of heteroplasmy, there is a threshold effect, whereby tissues function normally until the overall mutation load rises above a tissue-specific and often individual-specific level. Varying levels of heteroplasmy explain part of the phenotypical variation among siblings in the same family and between families with the same mutation [17].

The OXPHOS system consists of five multi-subunit complexes (complex I-V) and two electron carriers, coenzyme $\mathrm{Q}$ and cytochrome $\mathrm{c}$, situated in the mitochondrial inner membrane. All complexes of the OXPHOS system comprise subunits encoded in both the mitochondrial and the nuclear genome, except complex II which consists of subunits completely encoded by the nuclear DNA [18]. Complexes I and II reduce nicotinamide adenine dinucleotide (NADH) and flavin adenine dinucleotide $\left(F A D H_{2}\right)$, respectively. These reductions release electrons transported along the electron transport chain, which is accompanied by the generation of a proton gradient created by complexes I, III and IV. This proton gradient is used to generate ATP by the ATP synthase or complex V. 


\subsubsection{Complex I deficiency}

Complex I (NADH: ubiquinone oxidoreductase) is the largest OXPHOS complex, consisting of 45 subunits, of which seven are encoded by the mtDNA and 38 by the nDNA. Complex I dysfunction is the most common cause of OXPHOS disease in humans and accounts for approximately $30 \%$ of all OXPHOS disease [19]. The first pathogenic mutations in a nuclear gene causing human $\mathrm{Cl}$ deficiency was found in NDUFS8 [20]. To date, mutations have been identified in 21 complex I subunit genes, including all mtDNA encoded subunits and nine complex I assembly genes [21]. One of the most common clinical presentations associated with complex I deficiency is Leigh Syndrome. Leigh syndrome is characterized by a wide range of neurologic manifestations, including psychomotor delay and regression, muscular hypotonia, ataxia, dystonia, and ophthalmologic abnormalities (including nystagmus and vision loss). Clinical onset usually occurs by the age of 2 with symptoms often presenting during infection or illness after an initial period of normal development [22]. Adult onset Leigh syndrome has been infrequently reported [23]. Apart from Leigh syndrome, complex I deficiency can result in a wide range of clinical presentations with age at onset, ranging from neonatal to adult life, and including fatal lactic acidosis, neurodegeneration, hypotonia, ataxia, myopathy, cardiomyopathy, leukoencephalopathy, LHON and liver failure [21, 24, 25].

\subsubsection{Complex // deficiency}

Complex II (or succinate dehydrogenase) contains only four subunits, all nuclear encoded, and has a dual role in both OXPHOS and the TCA cycle. In the OXPHOS system, complex II transfers electrons to reduce ubiquinone, whereas in the TCA cycle complex II oxidizes the metabolite succinate to fumarate [26]. Many patients with Complex II deficiency present in childhood with optic atrophy and Leigh Syndrome with cardiomyopathy [27-31]. Conversely, adult onset cases of Complex II disorders are also known. Mutations in complex II subunit genes are rare, SDHA mutations are associated with Leigh syndrome, whereas $S D H B, S D H C$, and SDHD mutations appear to be a common cause of inherited paraganglioma and phaeochromocytoma. Mutations is the assembly factor gene SDHAF1 cause infantile leukoencephalopathy and an in SDHAF2 paraganglioma and pheochromocytoma $[28,32,33]$.

\subsubsection{Complex III deficiency}

Complex III (or cytochrome bc1 complex) is composed of 11 subunits, of which ten are encoded by the nuclear genome and one by the mtDNA (MT-CYB). Complex III transfers electrons from complex I and II to cytochrome c. Complex III deficiencies are relatively rare and express with a broad clinical variability. Clinical manifestations include lactic acidosis, LHON, sensorineural loss, liver failure, developmental delay, encephalopathy 
and cardiomyopathy [34-36]. Mutations in MT-CYB have been identified [37-39] whereas nuclear mutations have been found in two nuclear genes BCS1L and UQCRB $[35,40,41]$.

\subsubsection{Complex IV deficiency}

Complex IV (cytochrome c oxidase or COX) contains 13 subunits, of which ten are encoded by the nuclear genome and three by the mtDNA. These 3 mtDNA encoded subunits form the catalytic core of complex IV. Mutations in all mtDNA-encoded COX subunit genes have been identified, although these are relatively rare [42]. The majority of the pathogenic mutations leading to complex IV deficiency have been identified in nuclear genes, encoding structural subunits or assembly factors such as COX6B1, SURF1, PET100, COX10, COX15, LRPPRC and TACO1. Complex IV defects are associated with clinical phenotypes as myopathy, encephalopathy and Leigh syndrome [42].

\subsubsection{Complex V deficiency}

Complex $\mathrm{V}$, or ATP synthase, is the final enzyme of mitochondrial energy production by catalyzing the phosphorylation of ADP to ATP using the electrochemical gradient created by protons pumped into the mitochondrial intermembrane space during $\mathrm{Cl}$ to $\mathrm{CIV}$ electron transfer. Complex $V$ consists of two modules, the hydrophilic $F_{1}$ module faces the mitochondrial matrix and is involved in the synthesis and hydrolysis of ATP, whereas the hydrophobic $F_{0}$ module, spanning the inner mitochondrial membrane, transports protons back to the matrix from the intermembrane space [43]. Isolated complex $\mathrm{V}$ deficiency is the rarest of the OXPHOS disease. Most cases result from mutations in the mtDNA encoded MTATP6, which is associated with maternally inherited Leigh syndrome and neuropathy, ataxia, retinal pigmentosa (NARP). Additionally, mutations have been identified in nuclear encoding assembly factors, resulting in neonatal- onset hypertrophic cardiomyopathy, hypotonia, intrauterine growth restriction and oligohydramnios [43].

\subsubsection{Multiple OXPHOS complex deficiencies}

Multiple complex deficiencies are a common finding in OXPHOS diseases [44-46]. Combined defects of complexes I, III, IV and V can arise from a broad variety of gene mutations involved in mitochondrial DNA replication, RNA metabolism or translation (see 1.2.6.1). Complex I and IV appear to be the most vulnerable enzymes. This could be due to their larger number of mitochondrially encoded subunits, rendering them more vulnerable to mtDNA tRNA mutations, which are a frequent cause of mitochondrial disease. An example is the m.3243A>G tRNA-Leu mutation described in patients with complex I or IV deficiency and, as one of the manifestations, MELAS syndrome $[47,48]$. 
Also, defects in cofactors like coenzyme $Q$, iron-sulphur clusters, riboflavin and heme typically result in defects of more than one complex in various combinations. A combined defect of complex I and III has been due to SFXN4 mutations, affecting the mitochondrial inner membrane [49]. Finally, defects of mitochondrial homeostasis can cause a generalized decrease of all OXPHOS complexes. Mitochondrial homeostasis involves processes that regulate mitochondrial content and metabolism including lipid synthesis, protein import, fission and fusion (see 1.2.6.2), quality control and targeted degradation. Mutations in MFN2 involved in mitochondrial fusion result in multiple deletions in the mtDNA and multiple OXPHOS deficiencies [50].

\subsubsection{1 mtDNA replication, transcription and translation defects}

Defects in mtDNA replication, due to defects of the replication machinery or nucleotide metabolism can result in mtDNA deletions, point mutations, and/or depletion which ultimately cause OXPHOS deficiencies and mitochondrial disease. MtDNA is replicated by several nuclear encoded proteins and enzymes including DNA polymerase $\gamma(\mathrm{pol} \gamma)$, encoded by the POLG1 gene, its accessory subunit (pol $\gamma \mathrm{B}$ ), encoded by the POLG2 gene, the single-stranded DNA binding protein ( $\mathrm{mtSSB}$ ), the Twinkle helicase, encoded by PEO1, and a number of accessory proteins and transcription factors. Mutations have been detected in most of these genes, associated with diverse neurological disorders, including mtDNA depletion syndromes (MDS), Alpers-Huttenlocher syndrome [51], Progressive External Ophthalmoplegia (PEO) [52], SANDO syndrome (Sensory Ataxia, Neuropathy, Dysarthria, Ophthalmoparesis), mitochondrial neurogastrointestinal encephalomyopathy (MNGIE) [53] or Mitochondrial-associated Recessive Ataxia Syndrome (MIRAS) [54]. Additional to defects in mtDNA replication genes, mutations in genes involved in the salvage pathways of mitochondrial deoxynucleotide metabolism, like TP, ANT1, DGUOK, RRM2B and TK2, can lead to mtDNA deletions and depletion. This indicates that balanced nucleotide metabolism is critically important not only in replicating cells, but especially in quiescent (muscle) cells, which fully rely on the salvage pathway [55].

In general, mutations affecting the translation of mitochondrial proteins result in OXPHOS deficiencies affecting multiple complexes. The core mitochondrial transcription machinery contains the mitochondrial RNA polymerase (POLRMT) and transcription factor TFB2M. RNA transcription is regulated by mitochondrial transcription factor $A$ (TFAM) [56]. Termination of mature transcripts is carried out by mitochondrial termination factor 1 (MTERF1) [57]. The mitochondrial translation machinery is composed of mtDNA encoded tRNAs and rRNAs and a number of nuclear encoded proteins necessary for ribosomes assembly and function, being aminoacyl tRNA synthetases, tRNA modifying enzymes, factors of translation initiation (IF2-mt and IF- 3-mt), elongation (EFG1, EFG2, EFT, and EFTu), and termination, and release (mtRF1a, mtRF1, C12orf65 and ICT1). Defects of the mitochondrial protein synthesis usually cause clinical syndromes that are fatal or disabling, progressive, affecting the brain, heart, liver, skeletal muscle, 
and other organs with stereotypic features such as MELAS, PEO, MERRF (ragged red fibres), KSS (Kearns-Sayre syndrome) [58].

\subsubsection{Defective mitochondrial dynamics}

Mitochondria are dynamic organelles that change in number and morphology through mitochondrial fusion and fission. This process requires dynamins which are large GTPases with mechanochemical properties that remodel cellular membranes and also additional proteins, mostly involved in posttranslational alterations including phosphorylation, ubiquitylation and sumoylation, adding possible regulatory mechanisms [59]. Defects in mitochondrial dynamics can cause a number of neurological disorders (table 1).

Mitochondrial fusion depends on the GTPase activity of the mitofusin (MFN) family, mitofusins 1 and 2, which are present in the outer mitochondrial membrane. Mitochondrial fusion is initiated by MFN forming homo-oligomeric and hetero-oligomeric protein complexes between apposing mitochondria, tethering and fusing the outer membranes [60]. While mitofusin 1 and 2 regulate the fusion of the mitochondrial outer membrane, OPA1 mediates the inner membrane fusion [61].

Mitochondrial fission is mediated by FIS1 and the dynamin-related protein DRP1. FIS1 is anchored in the mitochondrial outer membrane by its C-terminal domain with two tandem tetratricopeptide repeat (TPR) domains directed to the cytoplasm, which mediate the interactions between FIS1 and other proteins [62]. Dynamin-related protein 1 (DRP1), residing as dimers and tetramers, acts as a mechano-enzyme. Once recruited by receptors on the mitochondrial outer membrane, it assembles into a helical ring which derives the constriction of mitochondria during division through GTP hydrolysis [63].

Tight regulation of the mitochondrial fission/fusion machinery proteins is essential for maintaining mitochondrial integrity and insure the presence of healthy mitochondria at critical locations. However, dysfunctional mitochondrial dynamics caused by an imbalance between mitochondrial fission and fusion is a hallmark of many neurodegenerative diseases and contributes to their pathophysiology. Crucial proteins in this respect are Mitofusin (Mfn) and Opa1, and Drp1 and Fis1 [64]. Genetic mutations in Mfn2 is the most common cause of Charcot-Marie-Tooth disease $[65,66]$. Mutations in Opa1 cause Autosomal Dominant Optic Atrophy type I (ADOA) or Chronic Progressive External Ophthalmoplegia (CPEO). The ADOA-plus syndrome also affects other body systems and can lead to deafness, dysphagia, myopathy, ataxia and peripheral neuropathy [67]. Mutation of Drp1 reported in a patient whose symptoms were broadly similar to those of CMT neuropathy and ADOA. 
Table 1 Human disorders associated with disturbed mitochondrial dynamics

\begin{tabular}{|c|c|c|}
\hline $\begin{array}{l}\text { Gene } \\
\text { Autosomal dominant }\end{array}$ & Function & Phenotypes \\
\hline Mitofusin 2 (MFN2) & OM fusion & $\begin{array}{l}\text { Charcot-Marie-Tooth disease axonal form type } \\
2 \mathrm{~A}(\mathrm{CMT} 2 \mathrm{~A})[65] \text {. Hereditary motor and sensory } \\
\text { neuropathy type } 6 \text { (HMSN6) }[50,68] \text {. }\end{array}$ \\
\hline Optic atrophy 1 (OPA1) & $\begin{array}{l}\text { IM fusion, cristae structure, anti- } \\
\text { apoptotic function }\end{array}$ & $\begin{array}{l}\text { Isolated optic atrophy and syndromic forms of } \\
\text { dominant optic atrophy }[67,69,70] \text {. }\end{array}$ \\
\hline $\begin{array}{l}\text { Ganglioside-induced } \\
\text { differentiation associated } \\
\text { protein } 1 \text { (GDAP1) }\end{array}$ & $\begin{array}{l}\text { Stimulation of DRP1-mediated } \\
\text { fission. Modulation of } \\
\text { mitochondrial mobility and } \\
\text { distribution }\end{array}$ & $\begin{array}{l}\text { Charcot-Marie-Tooth disease axonal form type } \\
2 \mathrm{~K}(\mathrm{CMT} 2 \mathrm{~K})[71] \text {. }\end{array}$ \\
\hline $\begin{array}{l}\text { Dynamin-related protein } \\
1(D R P 1)\end{array}$ & Mitochondrial fission & $\begin{array}{l}\text { Severe infantile neurodegenerative disease [ } 72 \text {, } \\
73] . \\
\text { Congenital microcephaly, lactic acidosis, sudden } \\
\text { death [72]. }\end{array}$ \\
\hline Optic atrophy 3 (OPA3) & & Optic atrophy and premature cataracts [74]. \\
\hline GDAP1 & & $\begin{array}{l}\text { Charcot-Marie-Tooth disease demyelinating } \\
\text { form type } 4 \text { A (CMT4A) [75]. }\end{array}$ \\
\hline OPA3 & & $\begin{array}{l}\text { 3-methylglutaconic aciduria type III (Costeff } \\
\text { syndrome) [76]. }\end{array}$ \\
\hline$S L C 25 A 46$ & $\begin{array}{l}\text { Interacts with Mgm1p and Fzo1p } \\
\text { to link IM and OM fusions } \\
\text { Ugo1p-like protein with pro-fission } \\
\text { activity }\end{array}$ & $\begin{array}{l}\text { Neuropathy, hereditary motor and sensory, } \\
\text { Leigh syndrome, lethal congenital } \\
\text { pontocerebellar hypoplasia [77-79]. }\end{array}$ \\
\hline
\end{tabular}

\subsubsection{Missing causes of mitochondrial disease}

Mitochondria play key roles not only in oxidative phosphorylation and energy metabolism, but also in ion homeostasis, small molecule metabolism, immune signaling and cell death [80]. To fully understand the molecular of mitochondrial pathways in health and disease, it is crucial to have a complete and accurate protein component list for this important organelle. In 2008, a comprehensive catalog of the mitochondrial proteome was made from the integration of multiple approaches. This source, termed MitoCarta, combined literature curation, large-scale GFP microscopy, and integration of extensive MS proteomics data with six other genome-scale data sets of mitochondrial localizations using a Bayesian framework [81]. However, the MitoCarta catalog is estimated to be only $85 \%$ complete with a $10 \%$ false positives and does not include many proteins that dually reside in mitochondrial and other cellular compartments [82]. At this moment, the human MitoCarta2.0 inventory includes 1158 mitochondrial protein-encoding genes, but approximately 300 genes have no association with a gene ontology (GO) biological process to indicate the function of the proteins [83]. A large number of uncharacterized mitochondrial proteins are associated with mitochondrial disorders, like for example, TMEM126B, a mitochondrial transmembrane protein required for the formation of complex I, found in patients with complex I deficiency (see chapter 6) [84, 
85]. TMEM126B was not part of the original MitoCarta, emphasizing the need to identify and annotate the missing $15 \%$ of the mitochondrial proteome.

\subsection{CLINICAL AND GENETIC HETEROGENEITY OF MITOCHONDRIAL DISEASE}

Clinical and genetic heterogeneity are the major hallmarks of mitochondrial disease. An extreme example of genetic heterogeneity is Leigh syndrome and to date, over 60 genes have been identified with mutations causing Leigh syndrome [86]. Most of these genes encode structural components of the OXPHOS complexes, or proteins required for assembly, stability, and maintenance of mtDNA integrity. The most frequently mutated gene in COX-deficient Leigh syndrome is the nuclear-encoded SURF1 gene [86]. Like other mitochondrial disease, Leigh syndrome has several possible modes of inheritance including autosomal dominant, autosomal recessive, and X-linked as well as maternal inheritance. Despite the remarkable progress in identifying causative gene defects, still many Leigh patients remain without a genetic diagnosis, suggesting that even more Leigh syndrome genes remain to be identified [86].

Mitochondrial disease are also clinically highly heterogeneous and different mutations in the same gene or even the same mutation can lead to different clinical outcomes. For example, manifestation of the m.3243A>G mutation in the mtDNA tRNA leucine gene can vary from being asymptomatic to severe MELAS syndrome, but also cardiomyopathy, deafness with diabetes, renal pathology and/or severe encephalopathy with death at young age have been described, either separately or in combination with other symptoms $[87,88]$. Genetic background, mutation loads, energy capacity and physical activity are possible causes of phenotypic variation [89]. Another issue is that some mtDNA mutations, even being homoplasmic, only affect one organ or tissue, like is the case in Leber's Hereditary Optic Neuropathy (LHON). The underlying pathogenic mechanism for this tissue specificity is not fully understood [90], but there are at least four possible factors which can influence phenotypic expression: environmental factors, mtDNA haplotype, nuclear DNA background, and tissue-specific expression of interacting genes [91].

\subsection{NON-MITOCHONDRIAL, NEUROLOGICAL DISEASES}

It is obvious that mitochondrial diseases are only a part of the spectrum of neurological diseases. Table 2-4 show clinical features as well as genetic causes of some nonmitochondrial neurological developmental disorders (table 2), hereditary movement (table 3) and neuromuscular and neurocutaneous disorders (table 4) [92]. In some of 
these, a mitochondrial gene defect could also be the underlying cause, explaining a subset of the cases.

Table 2: Examples of hereditary non-mitochondrial developmental disorders

\begin{tabular}{|c|c|c|c|c|}
\hline \multirow[b]{2}{*}{$\begin{array}{l}\text { Fragile X } \\
\text { syndrome }\end{array}$} & \multirow{2}{*}{$\begin{array}{l}\text { Prevalence } \\
\text { (1/4,000 in male } \\
\text { and } 1 / 6,000 \text { in } \\
\text { female) }\end{array}$} & \multirow{2}{*}{$\begin{array}{l}\begin{array}{l}\text { Genes or } \\
\text { chromosomes }\end{array} \\
\text { FMR1 }\end{array}$} & \multicolumn{2}{|c|}{ Inheritance pattern Clinical features } \\
\hline & & & X-linked & $\begin{array}{l}\text { Mental retardation, macro- } \\
\text { orchidism and distinct facial } \\
\text { features (long face, large ears, } \\
\text { prominent jaw) [93]. }\end{array}$ \\
\hline Rett syndrome & $1 / 10,000$ & MECP2 & X-linked & $\begin{array}{l}\text { Progressive autism, loss of } \\
\text { purposeful hand movements, } \\
\text { ataxia, acquired microcephaly, } \\
\text { gastrointestinal disorders and } \\
\text { seizures [94]. }\end{array}$ \\
\hline $\begin{array}{l}\text { Angelman } \\
\text { syndrome }\end{array}$ & $\begin{array}{l}1 / 20,000- \\
1 / 10,000\end{array}$ & UBE3A & $\begin{array}{l}\text { Sporadic cases, } \\
\text { autosomal } \\
\text { dominant }\end{array}$ & $\begin{array}{l}\text { Mental retardation, language } \\
\text { and cognition deficits, } \\
\text { movement or balance disorder } \\
\text { [95]. }\end{array}$ \\
\hline $\begin{array}{l}\text { Prader Willi } \\
\text { syndrome }\end{array}$ & $\begin{array}{l}1 / 25,000- \\
1 / 10,000\end{array}$ & $\begin{array}{l}\text { PWS/AS region } \\
\text { (15q11.2-q13) }\end{array}$ & Sporadic cases & $\begin{array}{l}\text { Obesity, short stature, muscular } \\
\text { hypotonia, mental retardation, } \\
\text { hypogonadotropic } \\
\text { hypogonadism, and small hands } \\
\text { and feet [96]. }\end{array}$ \\
\hline
\end{tabular}

Table 3: Examples of hereditary movement disorders

\begin{tabular}{|c|c|c|c|c|}
\hline & Prevalence & \multicolumn{2}{|c|}{ Genes or chromosomes Inheritance pattern } & Clinical features \\
\hline $\begin{array}{l}\text { Friedreich } \\
\text { ataxia }\end{array}$ & $1 / 25,000-1 / 50,000$ & FXN & Autosomal recessive & $\begin{array}{l}\text { Slowly progressive } \\
\text { ataxia, depressed } \\
\text { tendon reflexes, } \\
\text { dysarthria, Babinski } \\
\text { responses, and loss of } \\
\text { position and vibration } \\
\text { senses [97]. }\end{array}$ \\
\hline Dystonia & $7 / 1,000$ & TOR1A, GCH1. & $\begin{array}{l}\text { Autosomal dominant, } \\
\text { Autosomal recessive }\end{array}$ & $\begin{array}{l}\text { Involuntary, sustained } \\
\text { muscle contractions, } \\
\text { twisting movements } \\
\text { and abnormal postures } \\
\text { [98]. }\end{array}$ \\
\hline $\begin{array}{l}\text { Huntington's } \\
\text { disease }\end{array}$ & $5-10 / 100,000$ & HTT & Autosomal dominant & $\begin{array}{l}\text { Chorea, dystonia, } \\
\text { incoordination, } \\
\text { cognitive decline, and } \\
\text { behavioral difficulties } \\
\text { [99]. }\end{array}$ \\
\hline Wilson disease & $1 / 30,000$ & ATP7B & Autosomal recessive & $\begin{array}{l}\text { Neurological deficits, } \\
\text { liver cirrhosis [100]. }\end{array}$ \\
\hline
\end{tabular}


Table 4: Examples of hereditary neuromuscular and neurocutaneous disorders

\begin{tabular}{|c|c|c|c|c|}
\hline & Prevalence & $\begin{array}{l}\text { Genes or } \\
\text { chromosomes }\end{array}$ & $\begin{array}{l}\text { Inheritance } \\
\text { pattern }\end{array}$ & Clinical features \\
\hline $\begin{array}{l}\text { Charcot-Marie- } \\
\text { Tooth }\end{array}$ & $1 / 2,500$ & $\begin{array}{l}\text { Duplication of } \\
\text { chr.17p11.2, } \\
\text { involving PMP22, } \\
\text { EGR2, MPZ }\end{array}$ & $\begin{array}{l}\text { Autosomal } \\
\text { dominant, } \\
\text { Autosomal } \\
\text { recessive, } \\
\text { X-linked }\end{array}$ & $\begin{array}{l}\text { Distal muscle weakness and } \\
\text { atrophy, sensory loss, } \\
\text { depressed tendon reflexes, } \\
\text { and high-arched feet [101]. }\end{array}$ \\
\hline $\begin{array}{l}\text { Myotonic } \\
\text { dystrophy I \& II }\end{array}$ & $1 / 8,000$ & $D M P K \& C N B P$ & $\begin{array}{l}\text { Autosomal } \\
\text { dominant }\end{array}$ & $\begin{array}{l}\text { Myotonia, muscular } \\
\text { dystrophy, hypogonadism, } \\
\text { cataracts, cardiac } \\
\text { arrhythmias, diabetes [102]. }\end{array}$ \\
\hline \multicolumn{2}{|c|}{$\begin{array}{ll}\text { Duchenne/ Becker } 1 \text { in 3000-5000 } \\
\text { muscular } & \text { (Duchenne muscular } \\
\text { dystrophy } & \text { dystrophy) and } 1 \text { in } \\
& 18000 \text { (Becker variant) }\end{array}$} & DMD & X-linked & $\begin{array}{l}\text { Progressive muscle weakness } \\
\text { and reduced muscle tone } \\
{[103] \text {. }}\end{array}$ \\
\hline $\begin{array}{l}\text { Spinal muscular } \\
\text { atrophy type } 1\end{array}$ & $1 / 10000$ & SMN1 & $\begin{array}{l}\text { Autosomal } \\
\text { recessive }\end{array}$ & $\begin{array}{l}\text { degeneration of the anterior } \\
\text { horn cells of the spinal cord, } \\
\text { symmetrical muscle } \\
\text { weakness and atrophy [104]. }\end{array}$ \\
\hline \multicolumn{2}{|c|}{ Neurofibromatosis $1 / 4,000$} & NF1 & $\begin{array}{l}\text { Autosomal } \\
\text { dominant, } \\
\text { Sporadic cases }\end{array}$ & $\begin{array}{l}\text { Multiple cafe-au-lait spots, } \\
\text { Lisch nodules in the eye, skin } \\
\text { neurofibromas [105]. }\end{array}$ \\
\hline \multicolumn{2}{|c|}{ Tuberous sclerosis $1 / 15,000$} & TSC1, TSC2 & $\begin{array}{l}\text { Autosomal } \\
\text { dominant }\end{array}$ & $\begin{array}{l}\text { benign tumors and lesions in } \\
\text { multiple organ systems, } \\
\text { epilepsy, mental retardation, } \\
\text { and autism [106]. }\end{array}$ \\
\hline
\end{tabular}

\subsection{GENETIC TESTING IN MITOCHONDRIAL AND NEUROLOGICAL DISEASES}

A precise and complete understanding of the pathological mechanism underlying genetic neurological diseases will depend on finding the causative gene and damaging variants, causing the phenotype. In some cases, the genetic diagnosis of these disorders can be relatively straightforward based on, in case of mitochondrial diseases, the clinic in combination with biochemical or enzymatic defect by screening those genes directly related to these processes for mutations. This is the case for Leigh syndrome with complex IV deficiency, in which the Surf1 gene is the obvious candidate. Or in LHON, where $95 \%$ of the cases is explained by 3 mutations in the mtDNA. However, these are exceptions, and most diseases require a broad testing of many possible candidate genes, or even all genes, as still many new genes are likely to be involved.

At the start of the work, performed in this thesis, due to technical limitations stepwise phenotype-driven candidate gene screening was still the norm, but due to the rapid developments in sequencing technology, which will be discussed in the following paragraphs, mutation-driven screening of large numbers or even all genes are emerging 
as the prime genetic test for these genetically and clinically highly heterogeneous diseases, able to identify known and unknown genetic causes. Traditional Sanger-based testing is still being performed in disorders with distinctive phenotypes associated with minimal genetic heterogeneity or in case speed is an issue for example, for accurate treatment (thiamine to prevent Leigh syndrome due to SLC19A3 mutations) or to prevent side-effects of treatment (Valproate in case of POLG1 mutations). However, the advance of next-generation sequencing technology, allowing routine and cost-effective analysis of the entire coding part of the genome, provides a novel strategy to identify any genetic cause, irrespective of the clinical manifestation. This should enable identification of the genetic cause in the vast majority of genetic cases, especially suitable for single cases with many possible genetic causes. For example, in mitochondrial diseases any of more than 1500 genes might be involved, stressing the importance of unbiased, genome-wide approaches.

\subsection{ADVANCES IN SEQUENCING TECHNOLOGY}

In the 1970's, Maxam and Gilbert [107] and Sanger and colleagues [108] introduced methods to sequence the DNA, respectively, by chemically cleaving DNA or by incorporating dideoxy-nucleotides during DNA synthesis. Sanger sequencing became the golden standard for DNA sequencing for the following 30 years. In the 1990's, the Human Genome Project (HGP), an international effort to sequence and map the human genome, was performed, using highly automated versions of the Sanger sequencing method, eventually leading to the first draft version of the human genome, published in 2001 in Nature and Science [109]. Still, this method, despite having an accurate and reasonable read length (the actual number of continuous sequenced bases), has a low throughput and is too expensive to sequence a full genome in a diagnostic setting. For instance, sequencing the complete human genome with Sanger sequencing took 13 years of effort with an estimated cost of \$2.7 billion. The National Human Genome Research of the USA expressed the ambition to bring the cost of human genome sequencing down to $\$ 1000$ in ten years, which became feasible by the development of novel, massive parallel sequencing approaches, so-called next-generation sequencing or NGS.

NGS-platforms have a number of advantages over Sanger sequencing technology. Firstly, for Sanger sequencing the PCR amplified templates are sequenced together using chain terminating dye-deoxynucleotides to generate sufficient signal for detection [108]. In NGS, the DNA is sequenced from single molecules from a library, which are for example clustered (Illumina technology) and the DNA sequence is determined by continuously adding nucleotides to the complementary strand. With this approach, the sequence is derived from a single, amplified molecule [110]. Secondly, due to its high capacity, NGS is much faster in sequencing a large number of nucleotides compared to Sanger sequencing. In NGS, the clusters (Illumina technology) are sequenced simultane- 
ously in a massively parallel manner without any separation step allowing hundreds of millions of reactions on a single run on a single chip, whereas in Sanger sequencing, only one template of $500 \mathrm{bp}$ to $1 \mathrm{~kb}$ can be obtained per sequencing lane [111]. Thirdly, NGS is in contrast to Sanger sequencing also quantitative, and the relative amount of nucleotide variants can be determined, which is important for the analysis of mtDNA and tumor DNA $[112,113]$. This also allows other applications, like transcriptomics (RNA-seq), generating both quantitative and qualitative data of the expressed genes [114].

A disadvantage of most NGS technologies is their relatively shorter read length compared to Sanger sequence (Table 1). This becomes critical for mapping the reads to a reference sequence. The deeper coverage of NGS partly compensates the shorter read length. However, that depth of coverage cannot always overcome problems, associated with highly polymorphic or repetitive regions and, for example, de novo assembly is difficult [115].

Table 5:

\begin{tabular}{ll}
\hline NGS technology & Read length in bases \\
\hline Sanger & $\sim 800$ \\
Roche/454 & $\sim 450$ \\
Illumina/Solexa & $36-100$ \\
ABI/SOLiD & $35-50$ \\
Polonator & 26 \\
Minion & 8,000 \\
PacBio & 10,000 \\
\hline
\end{tabular}

In 2005, the first commercially available NGS technology was introduced (Roche/454 genome sequencer, 454 Life Sciences, Branford, CT). Parallelization was obtained through rapid amplification of small, bead-bound fragments of DNA using emulsionPCR. The sequencing reaction utilizes a technique called pyrosequencing. This system was capable of generating read lengths of 100 bases and the current generation reaches read lengths of $>400$ bases [116]. Two other NGS platforms, the Solexa/Illumina sequencing and the SOLiD system, were released a few years later in 2007. Illumina developed a technique known as bridge amplification to clone fragments of singlestranded DNA immobilized in a flow cell. The sequencing occurs when fluorescently labeled nucleotides attach to the target strand one at a time (see 1.6.1).

Recently, technologies such as single-molecule real-time sequencing (SMRT, PaCBIO and Minion, Nanopore) avoid the amplification step, minimizing the risk of introducing substitutions and/or bias during amplification, and provide sequence data for individual template molecules $[117,118]$. However, the error rate of the single-molecule sequencing technology itself is still much higher than for the other platforms. To generate sequence data, MinION sequencer creates electrical current measurements as DNA molecules pass through pores, by which the sequence is being determined [119]. In such a 
run, both strands will be read and a consensus sequence of the molecule can be produced (Figure 3). SMRT sequencing harnesses the natural process of DNA replication and enables real-time observation of DNA synthesis (see 1.6.2). In addition, SMRT sequencing has also been able to identify DNA modifications, such as different types of methylation [120], in contrast to only cytosine methylation which most high-throughput techniques focus on [120]. The single molecule sequencers were referred to as third generation sequencing technologies. The emergence and development third generation sequencing platforms such SMRT sequencing has permitted several thousands of up to several kilo bases long reads to be generated [121]. Third generation sequencing has greatly improved the genome assembly and structural variation analysis. Illumina, being the market leader in NGS, and SMRT sequencing (PacBioSystems) will be described in more detail below.

\subsubsection{Illumina sequencing (Solexa) GA/HiSeq/MiSeq/NextSeq/NovaSeq}

Illumina sequencers are the most widely used systems nowadays. Due the larger throughput at lower costs, many customers have switched from 454 to Illumina platforms over the years [122-124]. The Illumina technology is different from 454 in several aspects: the surface of the flow cell is coated with oligonucleotides, instead of a microwell chip containing beads, and it is based on reversible dye terminators rather than pyrosequencing. No emulsion PCR is required. To be precise, the library preparation begins with forward and reverse oligonucleotides hybridize to the ends of the fragments (figure 2). Each fragment is amplified to form a cluster of identical fragments. Next, the templates are sequenced by synthesis technology using fluorescent labeled nucleotides in a massively parallel fashion. Although the Illumina sequencing technology is very similar to Sanger sequencing, reversible dye terminators are removed after introduction into DNA strands, allowing further sequencing of the same molecule and quantitative sequencing by synthesis [125]. Currently, Illumina's Miseq produces the longest reads of up to $2 \times 300 \mathrm{bp}$. The NovaSeq series provides access to a higher throughput than ever before at a lower entry price. The NovaSeq 6000 system, available in 2017, can process up to $3000 \mathrm{~Gb}$ in a paired-end fashion with 150 bases for each read. 


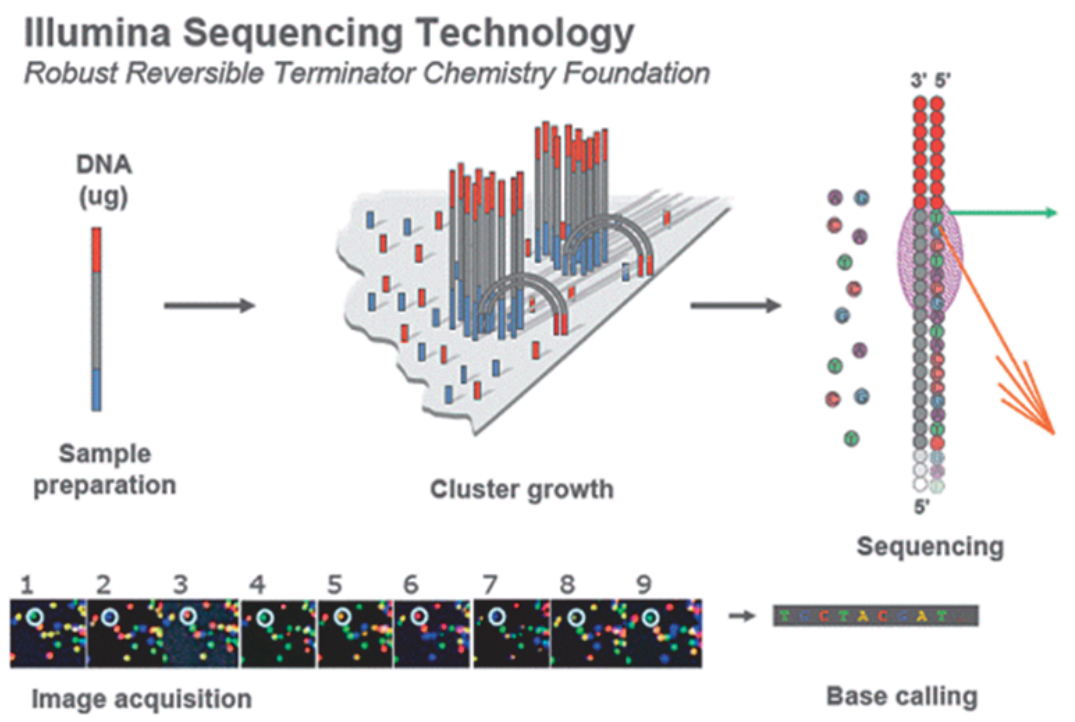

Figure 2: Illumina sequencing technology. Fluorescent-labelled and reversible terminator nucleotides, a polymerase and adapter-modified, single-stranded DNA are added. Attached DNA fragments are amplified forming "bridge" molecules. Base incorporation is followed by an imaging step and the Fluor and block are removed before the next synthesis cycle. The analysis software then processes the captured images into a sequence. (www.illumina.com)

\subsubsection{SMRT-sequencing - Pacific Biosciences (PacBio)}

Although Helicos BioSciences was the first implementation of single-molecule sequencing platform, Pacific Biosciences (PacBio) is currently leading the way for single molecule sequencing [126]. When PacBio first launched SMRT sequencing, it received a mixed response due to concerns on its inaccuracy in base calls and lower throughput. However, upgrades to their system of circular consensus sequencing (CCS; also called reads of insert) have improved sequence accuracy, allowing repeated sequencing and thereby reducing error rates [127]. Unlike other next generation sequencing systems, PacBio does not require an amplification step. The sequencing is done on a chip that contains many zero-mode waveguide (ZMW) detectors (figure 3). DNA polymerases are affixed to the ZMW detectors and the fluorescent signal of the nucleotide incorporation is imaged in real time observation of DNA synthesis. PacBio's RS II C2 XL is capable of generating long reads averaging around 4,600 bases and about 47,000 reads per run [127]. 

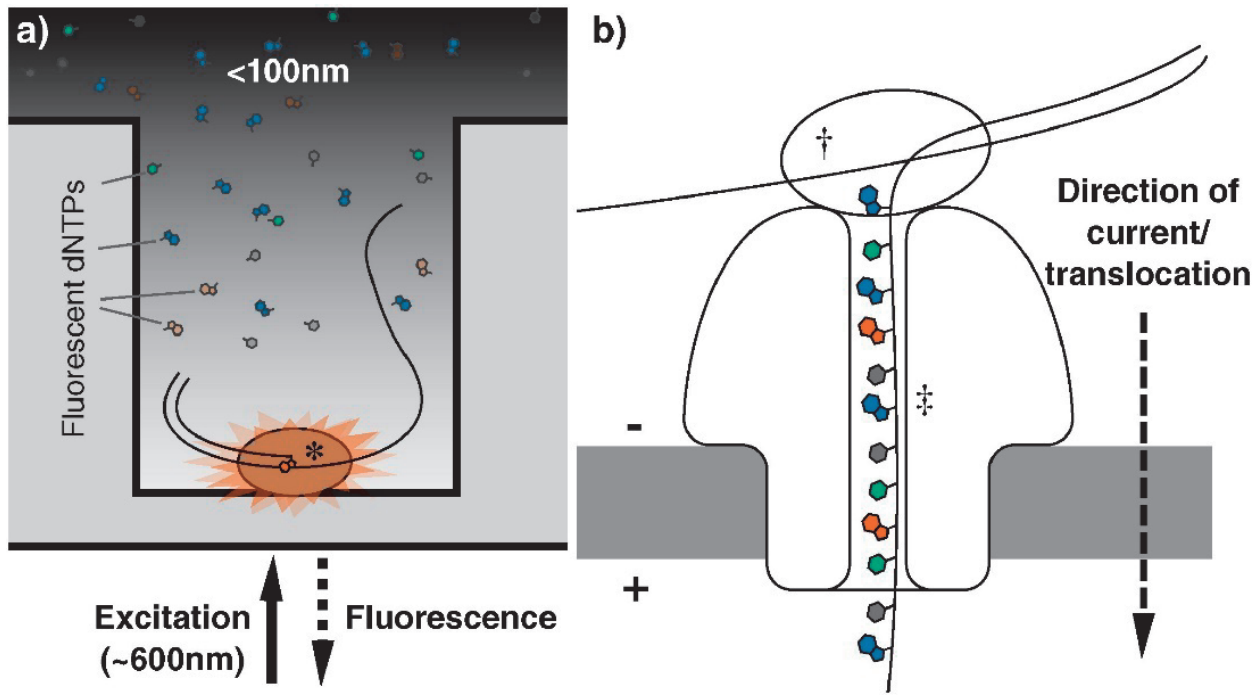

Figure 3: Third generation sequencing platforms. (a) PacBio SMRT sequencing: DNA polymerase is immobilized at the bottom of each ZMW. Target DNA and fluorescent-labeled nucleotides are added, allowing continuous polymerization of the template at the base. Base incorporation provides real-time bursts of detectable fluorescent signal. (b) Nanopore DNA sequencing employed in MinION sequencer: Double stranded DNA gets denatured into two strands. Single stranded DNA is fed through a pore in the membrane, altering the current in a distinctive manner. The sequence is inferred by analysis of fluctuations in the ionic flow [128].

\subsubsection{NGS in the clinic}

NGS has entered the clinic, not only for the detection of the causative mutation in genetic disease, but also for the identification of driver mutations in cancer and for typing pathological micro-organisms. In genetic diseases, dependent on the clinical symptoms, either a candidate-gene based approach is applied or an unbiased whole-exome or whole-genome strategy. In the candidate-gene approach only genes are being sequenced which are known to be able to explain the clinical symptoms [129]. The advantage is that the number of variants to be analyzed is limited and that unsolicited findings do not occur. A disadvantage is that novel genes will not be identified nor gene defects in known genes with an unknown clinical manifestation. In whole exome sequencing the protein-coding regions, representing 1-2\% of the genome, are being analyzed. The exome accounts for about $85 \%$ of disease mutations [130]. WES has been used the last couple of years with great success to identify known and novel gene defects and genes in individuals with neurological disease [131]. A disadvantage is the high number of variants, that need to be interpreted or in case of unknown genes, functionally validated, including many variants of unknown significance, and the possibility to detect unsolicited findings, for example pathogenic mutations in genes not related to the phenotype. Whole genome sequencing has the potential to identify nearly all forms of genetic variation, but the disadvantages of whole exome sequencing are amplified. 
Irrespective of the current situation, it is evident that, when the costs drop, whole genome sequencing will become the method of choice for mutation detection in genetically heterogeneous Mendelian diseases and at a later stage for the entire population to enable personalized medicine [132-135].

\subsection{FUNCTIONAL STUDIES}

One of the major challenges in the application of exome/whole genome sequencing approaches is the functional validation and clinical interpretation of variants. Although bioinformatic tools are useful at several stages of filtering and prioritizing candidate genes as well as predictions on the impact of coding variants on protein function, they remain insufficient to prove causality for, or contribution to the phenotype. Therefore, models (table 6) are required to provide support for a causal role for any candidate gene and variant of unknown significance. A cell culture system is the easiest model and suitable for investigating a disorder defined on the basis of a biochemical or metabolic deficiency. However, if a disorder is associated with developmental, behavioral or physiological defects, genes and variants should be validated in in vivo model.

\subsubsection{Patient-derived cell lines}

Patient-derived cell lines, like fibroblasts, are frequently used, especially in OXPHOS disease, where a biochemical defect in the OXPHOS system can be measured. OXPHOS assembly studies can be performed and mitochondrial networks studied. The application of skin fibroblast as an in vitro model is partly based upon the ease of obtaining, culturing and maintaining. They can be grown in larger amounts and are stable for many generations. The rationale for these experiments is the causative relation between the biochemical defects, as also observed in the cell lines, and the clinical manifestations [136]. In patients with mitochondrial disorders, functional complementation studies restoring biochemical phenotype in fibroblasts with the wild-type gene, but not with the mutated gene supports the pathogenicity of the mutations [137].

\subsubsection{Induced pluripotent cell lines (iPSC)}

In 2006, the field of somatic cell reprogramming advanced by a landmark study of producing induced pluripotent stem cells (iPSCs) from somatic cells by Yamanaka and coworkers. These iPSCs possess pluripotency, self-renewal, and most key characteristics of embryonic stem cells $[138,139]$. The pluripotent state has been induced in a variety of human somatic cells types including fibroblasts, keratinocytes and lymphocytes [140]. iPSC-based neurological disease models from patient- specific cells have identified molecular lesions, which could be related to the pathology observed [141-143]. Compared 
to fibroblasts, the iPSC-derived differentiated cells have the major advantage of allowing the study of cell- or tissue-specific expression, which may be a key factor in the pathology of tissue-specific expressed genes and would allow testing therapies in the relevant tissue-type.

In case cell line model are not suitable, animal models might provide an alternative. It is not that one single model will suffice for all pathologies observed and the appropriate model should be carefully selected based on being the best choice for the specific question addressed.

\subsubsection{Drosophila melanogaster}

Drosophila melanogaster or the fruit fly has many advantages to study molecular and cellular mechanisms. Drosophila has a short life cycle, rapid reproduction and the anatomical structures are well known. Moreover, an extensive toolbox is available for Drosophila to knock down genes in a time- and tissue- specific manner [144] and it is relative easy to introduce mutations in Drosophila genes. These tools are very useful for the functional characterization of genes and the study of genetic defects in neurological disorders [145-147]. Despite the fact that key molecular pathways are well conserved between Drosophila and humans, there are still significant anatomical differences between fruit fly and humans, which may be not sufficient to recapitulate specific aspects of neurological disease. In that respect, a vertebrate model organism would be more suitable.

\subsubsection{Danio rerio}

Danio rerio or zebrafish has become one of the favored animal models. Besides being a vertebrate with common organs and tissues such as a brain and spinal cord with conserved organization, zebrafish share high genetic similarity to humans with $50-80 \%$ homology with most human sequences [148]. The major advantages of the zebrafish as a model are the relative simplicity of manipulating gene expression in living embryos with relevance to human pathology and the large numbers of embryos, which can be generated and analyzed efficiently, cost effective and in a short time. Another advantage is that they are transparent during early development, making embryological studies extremely powerful. A complication is the fact that zebrafish contains a large number of duplicated genes, complicating identifying the functional homologue and gene editing strategies. This phenomenon occurred due to a whole genome duplication occurred during evolution [149]. 


\subsubsection{Mus musculus}

Transgenesis and gene targeting in Mus musculus or the mouse are powerful and widely used tools in the study of human gene function. Investigating human genetic diseases in the mouse provides the opportunity to elucidate the pathogenesis of the disease and proposes therapeutic approaches for human patients. There is high degree of similarity between the genomes of humans and mice and less than $1 \%$ of mouse genes has no homology to a human gene. Homologous genes are found in conserved syntenic regions in preserved order and relative orientation [150]. A large panel of techniques available in mouse genetics allowing very precise genomic manipulation [151].

Table 6: Overview of different models and their advantages/ disadvantages.

\begin{tabular}{|c|c|c|c|}
\hline Methods & Examples & Advantages & Disadvantages \\
\hline \multirow[t]{4}{*}{ In vivo } & Caenorhabditis elegans & $\begin{array}{l}\text { Focus on cellular level without } \\
\text { systemic complexity }\end{array}$ & Lacks spinal cord, neurons \\
\hline & Drosophila & $\begin{array}{l}\text { More complex features than } \\
\text { C.elegans, but less than vertebrates }\end{array}$ & $\begin{array}{l}\text { Relatively simplistic compared } \\
\text { with human, brain anatomy } \\
\text { differs substantially from humans }\end{array}$ \\
\hline & Zebrafish & $\begin{array}{l}\text { External embryos manipulations } \\
\text { possible, transparent, simple } \\
\text { vertebrate, can develop transgenic } \\
\text { and knockout animals, genome } \\
\text { analysis well under way (good SNP } \\
\text { and linkage maps) }\end{array}$ & $\begin{array}{l}\text { Not mammalian; duplication of } \\
\text { genome }\end{array}$ \\
\hline & Mouse & $\begin{array}{l}\text { Mammalian model, large mutant } \\
\text { collection }\end{array}$ & $\begin{array}{l}\text { Expensive and time consuming. } \\
\text { Pathology and behavior only } \\
\text { develop in some lines and at an } \\
\text { advanced age. }\end{array}$ \\
\hline \multirow[t]{2}{*}{$\begin{array}{l}\text { In vitro } \\
\text { cultures }\end{array}$} & Yeast & $\begin{array}{l}\text { short generation, successfully } \\
\text { employed as a high-throughput } \\
\text { genetic and small molecule screen. }\end{array}$ & $\begin{array}{l}\text { Not systemic, neural, or even } \\
\text { human }\end{array}$ \\
\hline & IPSCS, fibroblasts & $\begin{array}{l}\text { Realistic model of human disease, } \\
\text { pluripotent and unlimited supply. }\end{array}$ & $\begin{array}{l}\text { Partially immature phenotype. } \\
\text { Uncontrolled viral integration into } \\
\text { genome during iPSC derivation } \\
\text { poses limitations for } \\
\text { comprehensive conclusions. }\end{array}$ \\
\hline
\end{tabular}

\subsubsection{Conclusion functional models}

A large number of animal and cellular models are available for in-depth studies of neurological and mitochondrial disorders. These models play an important role in providing accurate and experimentally accessible systems to study the mechanisms of pathogenesis and to test potential therapeutic treatment strategies. Examples are mouse models of nuclear encoded complex I subunit NDUFS4 and cytochrome c $[152,153]$ or zebrafish models for Cerebellar Ataxia (CA), Hereditary Spastic Paraplegia (HSP) and CharcotMarie Tooth Disease (CMT) [154-156]. Neurological disorders, particularly those related 
to inborn errors of metabolism, such as Lesch-Nyhan syndrome, lysosomal storage disorders, lipidoses and OXPHOS disease, have been identified by means of biochemical and genetic studies of cultured fibroblasts [157]. In the long run, animal and cellular models are great source for a better understanding of neurological diseases mechanisms, drug discovery and future therapeutic applications.

\subsection{OUTLINE OF THE THESIS}

Sanger sequencing is a highly sensitive and straightforward tool to identify mutations in specific genes. However, in genetically heterogeneous disorders, in which many genes can be involved and not all candidate genes are yet identified, this approach falls short by the lack of throughput. The diagnostic yield of Sanger-based sequencing in OXPHOS diseases is about 25\%. Next generation sequencing has the potential to detect any variant in the human genome or exome in a rapid and cost-effective manner. Being an unbiased approach, no prior knowledge on genotype-phenotype correlations are required, only the genetic model has to be predefined for filtering of the variants. Only rare variants matching the genetic model and damaging the protein are being further evaluated for a causal role in the phenotype. Whole exome sequencing has the additional advantage of being able to identify multiple genetic causes, as complex clinical phenotypes are not necessarily single gene disorders, but can be caused by mutations in multiple genes.

The central aim of this thesis is to identify and functionally characterize gene defects in genetic neurological disorders, in most cases with an a priori possible mitochondrial involvement, using whole exome sequencing and specific functional models. Finding the genetic defect, not only will improve diagnosis and counseling, but will also reveal new pathological mechanisms and treatment options.

In chapter 2 to 5 we describe the identification and functional characterization of defects in novel disease genes in 4 families with autosomal recessive disease. Chapter 2 reports a patient from non-consanguineous Dutch parents, with cerebellar ataxia and atrophy, who was compound heterozygous for pathogenic variants in the CWF19L1 gene. Chapter 3 describes a Moroccan girl of consanguineous parents with optic atrophy and cerebellar atrophy, in whom we detected a homozygous pathogenic SLC25A46 mutation. The mutation was shown to affect mitochondrial integrity. Our findings support SLC25A46 mutations as a novel cause for optic atrophy spectrum disorder in patients with optic atrophy and cerebellar atrophy. Chapter 4 described a patient from non-consanguineous Dutch parents, with isolated COX deficiency, cerebellar atrophy and type 1 diabetes, who was compound heterozygous for two splice site variants in the COX18 gene. Both mutations were shown to result in altered splicing. Our results indicate COX18 mutations as a novel cause of isolated COX deficiency. Chapter 5 described a Turkish patient from a consanguineous family with a severe neurodegenerative condi- 
tion. WES combined with homozygosity mapping identified mutations in 2 candidate genes, HKDC1 and MED20. We investigated the role of HKDC1 in the phenotype by morpholino (MO) knockdown experiments in zebrafish embryos. Chapter 6 gives an overview of all gene and gene defects identified in a cohort of 119 patients with a mitochondrial defect as a likely or possible cause. For this cohort, a next generation sequencing based approach was used in two steps. First, the mtDNA was screened for mutations, and secondly, if negative, WES was performed. Our results showed an overall yield of $\sim 70 \%$ in (suspected) mitochondrial disorders using whole mtDNA and exome sequencing. Interestingly, $31 \%$ of the disease-causing genes in these patients were at the date of genetic diagnosis not present in the MitoCarta database. Our results illustrate that WES is a comprehensive and unbiased approach to establish a genetic diagnosis in mitochondrial disease. In Chapter 7, the general discussion, we discuss different approaches of NGS and their strengths and weaknesses in the context of genetically diagnosing neurological disorders and developing new gene-based treatment options for these disorders. The possible animal and cell culture models of neurological disorders will be presented in further detail, highlighting the pros and cons of each model. 


\section{REFERENCES}

1. Faghihi, M.A., S. Mottagui-Tabar, and C. Wahlestedt, Genetics of neurological disorders. Expert Rev Mol Diagn, 2004. 4(3): p. 317-32.

2. Organization, W.H., Neurological disorders: Public Health Challenges. 2006.

3. Simonato, M., et al., Progress in gene therapy for neurological disorders. Nat Rev Neurol, 2013. 9(5): p. 277-91.

4. Gray, M.W., Mitochondrial evolution. Cold Spring Harb Perspect Biol, 2012. 4(9): p. a011403.

5. McBride, H.M., M. Neuspiel, and S. Wasiak, Mitochondria: more than just a powerhouse. Curr Biol, 2006. 16(14): p. R551-60.

6. Tuppen, H.A., et al., Mitochondrial DNA mutations and human disease. Biochim Biophys Acta, 2010. 1797(2): p. 113-28.

7. Khan, N.A., et al., Mitochondrial disorders: challenges in diagnosis \& treatment. Indian J Med Res, 2015. 141(1): p. 13-26.

8. Sato, M. and K. Sato, Maternal inheritance of mitochondrial DNA by diverse mechanisms to eliminate paternal mitochondrial DNA. Biochim Biophys Acta, 2013. 1833(8): p. 1979-84.

9. Lightowlers, R.N., R.W. Taylor, and D.M. Turnbull, Mutations causing mitochondrial disease: What is new and what challenges remain? Science, 2015. 349(6255): p. 1494-9.

10. Skladal, D., J. Halliday, and D.R. Thorburn, Minimum birth prevalence of mitochondrial respiratory chain disorders in children. Brain, 2003. 126(Pt 8): p. 1905-12.

11. Gorman, G.S., et al., Prevalence of nuclear and mitochondrial DNA mutations related to adult mitochondrial disease. Ann Neurol, 2015. 77(5): p. 753-9.

12. McFarland, R., R.W. Taylor, and D.M. Turnbull, The neurology of mitochondrial DNA disease. Lancet Neurol, 2002. 1(6): p. 343-51.

13. Anderson, S., et al., Complete sequence of bovine mitochondrial DNA. Conserved features of the mammalian mitochondrial genome. J Mol Biol, 1982. 156(4): p. 683-717.

14. Shadel, G.S. and D.A. Clayton, Mitochondrial DNA maintenance in vertebrates. Annu Rev Biochem, 1997. 66: p. 409-35.

15. Smeets, H.J., Preventing the transmission of mitochondrial DNA disorders: selecting the good guys or kicking out the bad guys. Reprod Biomed Online, 2013. 27(6): p. 599-610.

16. St John, J. and R. Lovell-Badge, Human-animal cytoplasmic hybrid embryos, mitochondria, and an energetic debate. Nat Cell Biol, 2007. 9(9): p. 988-92.

17. Chinnery, P.F., et al., Molecular pathology of MELAS and MERRF. The relationship between mutation load and clinical phenotypes. Brain, 1997. 120 ( Pt 10): p. 1713-21.

18. Anderson, S., et al., Sequence and organization of the human mitochondrial genome. Nature, 1981. 290(5806): p. 457-65.

19. Nsiah-Sefaa, A. and M. McKenzie, Combined defects in oxidative phosphorylation and fatty acid betaoxidation in mitochondrial disease. Biosci Rep, 2016. 36(2).

20. Loeffen, J., et al., The first nuclear-encoded complex I mutation in a patient with Leigh syndrome. Am J Hum Genet, 1998. 63(6): p. 1598-608.

21. Mimaki, M., et al., Understanding mitochondrial complex I assembly in health and disease. Biochim Biophys Acta, 2012. 1817(6): p. 851-62.

22. Leigh, D., Subacute necrotizing encephalomyelopathy in an infant. J Neurol Neurosurg Psychiatry, 1951. 14(3): p. 216-21.

23. Chalmers, R.M., et al., A mitochondrial DNA tRNA(Val) point mutation associated with adult-onset Leigh syndrome. Neurology, 1997. 49(2): p. 589-92.

24. Komen, J.C. and D.R. Thorburn, Turn up the power - pharmacological activation of mitochondrial biogenesis in mouse models. Br J Pharmacol, 2014. 171(8): p. 1818-36.

25. Rotig, A. and A. Munnich, Genetic features of mitochondrial respiratory chain disorders. J Am Soc Nephrol, 2003. 14(12): p. 2995-3007. 
26. Cecchini, G., Function and structure of complex II of the respiratory chain. Annu Rev Biochem, 2003. 72: p. 77-109.

27. Finsterer, J. and S. Zarrouk-Mahjoub, Diagnosis of Kearns-Sayre Syndrome Requires Comprehensive Work-up. Chin Med J (Engl), 2016. 129(20): p. 2518-2519.

28. Ghezzi, D., et al., SDHAF1, encoding a LYR complex-II specific assembly factor, is mutated in SDHdefective infantile leukoencephalopathy. Nat Genet, 2009. 41(6): p. 654-6.

29. Bugiani, M., et al., Effects of riboflavin in children with complex II deficiency. Brain Dev, 2006. 28(9): p. 576-81.

30. Bourgeron, T., et al., Mutation of a nuclear succinate dehydrogenase gene results in mitochondrial respiratory chain deficiency. Nat Genet, 1995. 11(2): p. 144-9.

31. Alston, C.L., et al., A recessive homozygous p.Asp92Gly SDHD mutation causes prenatal cardiomyopathy and a severe mitochondrial complex II deficiency. Hum Genet, 2015. 134(8): p. 869-79.

32. Ohlenbusch, A., et al., Leukoencephalopathy with accumulated succinate is indicative of SDHAF1 related complex II deficiency. Orphanet J Rare Dis, 2012. 7: p. 69.

33. Bayley, J.P., et al., SDHAF2 mutations in familial and sporadic paraganglioma and phaeochromocytoma. Lancet Oncol, 2010. 11(4): p. 366-72.

34. Brown, M.D., et al., Mitochondrial DNA complex I and III mutations associated with Leber's hereditary optic neuropathy. Genetics, 1992. 130(1): p. 163-73.

35. de Lonlay, P., et al., A mutant mitochondrial respiratory chain assembly protein causes complex III deficiency in patients with tubulopathy, encephalopathy and liver failure. Nat Genet, 2001. 29(1): p. 57-60.

36. Nogueira, C., et al., Novel TTC19 mutation in a family with severe psychiatric manifestations and complex III deficiency. Neurogenetics, 2013. 14(2): p. 153-60.

37. Johns, D.R. and M.J. Neufeld, Cytochrome b mutations in Leber hereditary optic neuropathy. Biochem Biophys Res Commun, 1991. 181(3): p. 1358-64.

38. Andreu, A.L., et al., Exercise intolerance due to mutations in the cytochrome $b$ gene of mitochondrial DNA. N Engl J Med, 1999. 341(14): p. 1037-44.

39. De Coo, I.F., et al., A 4-base pair deletion in the mitochondrial cytochrome b gene associated with parkinsonism/MELAS overlap syndrome. Ann Neurol, 1999. 45(1): p. 130-3.

40. Schuelke, M., et al., Septo-optic dysplasia associated with a new mitochondrial cytochrome $b$ mutation. Ann Neurol, 2002. 51(3): p. 388-92.

41. Visapaa, I., et al., GRACILE syndrome, a lethal metabolic disorder with iron overload, is caused by a point mutation in BCS1L. Am J Hum Genet, 2002. 71(4): p. 863-76.

42. Shoubridge, E.A., Cytochrome c oxidase deficiency. Am J Med Genet, 2001. 106(1): p. 46-52.

43. Jonckheere, A.I., J.A. Smeitink, and R.J. Rodenburg, Mitochondrial ATP synthase: architecture, function and pathology. J Inherit Metab Dis, 2012. 35(2): p. 211-25.

44. Scaglia, F., et al., Clinical spectrum, morbidity, and mortality in 113 pediatric patients with mitochondrial disease. Pediatrics, 2004. 114(4): p. 925-31.

45. Gibson, K., et al., Mitochondrial oxidative phosphorylation disorders presenting in neonates: clinical manifestations and enzymatic and molecular diagnoses. Pediatrics, 2008. 122(5): p. 1003-8.

46. Honzik, T., et al., Neonatal onset of mitochondrial disorders in 129 patients: clinical and laboratory characteristics and a new approach to diagnosis. J Inherit Metab Dis, 2012. 35(5): p. 749-59.

47. Zierz, C.M., P.R. Joshi, and S. Zierz, Frequencies of myohistological mitochondrial changes in patients with mitochondrial DNA deletions and the common m.3243A>G point mutation. Neuropathology, 2015. 35(2): p. 130-6.

48. Santorelli, F.M., et al., Maternally inherited encephalopathy associated with a single-base insertion in the mitochondrial tRNATrp gene. Ann Neurol, 1997. 42(2): p. 256-60.

49. Hildick-Smith, G.J., et al., Macrocytic anemia and mitochondriopathy resulting from a defect in sideroflexin 4. Am J Hum Genet, 2013. 93(5): p. 906-14.

50. Rouzier, C., et al., The MFN2 gene is responsible for mitochondrial DNA instability and optic atrophy 'plus' phenotype. Brain, 2012. 135(Pt 1): p. 23-34. 
51. Naviaux, R.K. and K.V. Nguyen, POLG mutations associated with Alpers' syndrome and mitochondrial DNA depletion. Ann Neurol, 2004. 55(5): p. 706-12.

52. Van Goethem, G., et al., Mutation of POLG is associated with progressive external ophthalmoplegia characterized by mtDNA deletions. Nat Genet, 2001. 28(3): p. 211-2.

53. Wong, L.J., et al., Molecular and clinical genetics of mitochondrial diseases due to POLG mutations. Hum Mutat, 2008. 29(9): p. E150-72.

54. Hakonen, A.H., et al., Mitochondrial DNA polymerase W748S mutation: a common cause of autosomal recessive ataxia with ancient European origin. Am J Hum Genet, 2005. 77(3): p. 430-41.

55. Fasullo, M. and L. Endres, Nucleotide salvage deficiencies, DNA damage and neurodegeneration. Int J Mol Sci, 2015. 16(5): p. 9431-49.

56. Metodiev, M.D., et al., Methylation of $12 \mathrm{~S}$ rRNA is necessary for in vivo stability of the small subunit of the mammalian mitochondrial ribosome. Cell Metab, 2009. 9(4): p. 386-97.

57. Gaspari, M., N.G. Larsson, and C.M. Gustafsson, The transcription machinery in mammalian mitochondria. Biochim Biophys Acta, 2004. 1659(2-3): p. 148-52.

58. Scheper, G.C., M.S. van der Knaap, and C.G. Proud, Translation matters: protein synthesis defects in inherited disease. Nat Rev Genet, 2007. 8(9): p. 711-23.

59. Praefcke, G.J. and H.T. McMahon, The dynamin superfamily: universal membrane tubulation and fission molecules? Nat Rev Mol Cell Biol, 2004. 5(2): p. 133-47.

60. Koshiba, T., et al., Structural basis of mitochondrial tethering by mitofusin complexes. Science, 2004. 305(5685): p. 858-62.

61. Song, Z., et al., OPA1 processing controls mitochondrial fusion and is regulated by mRNA splicing, membrane potential, and Yme1L. J Cell Biol, 2007. 178(5): p. 749-55.

62. Dohm, J.A., et al., Cytosolic domain of the human mitochondrial fission protein fis1 adopts a TPR fold. Proteins, 2004. 54(1): p. 153-6.

63. van der Bliek, A.M., A mitochondrial division apparatus takes shape. J Cell Biol, 2000. 151(2): p. F1-4.

64. Youle, R.J. and M. Karbowski, Mitochondrial fission in apoptosis. Nat Rev Mol Cell Biol, 2005. 6(8): p. 65763.

65. Zuchner, S., et al., Mutations in the mitochondrial GTPase mitofusin 2 cause Charcot-Marie-Tooth neuropathy type 2A. Nat Genet, 2004. 36(5): p. 449-51.

66. Kijima, K., et al., Mitochondrial GTPase mitofusin 2 mutation in Charcot-Marie-Tooth neuropathy type 2A. Hum Genet, 2005. 116(1-2): p. 23-7.

67. Delettre, C., et al., Nuclear gene OPA1, encoding a mitochondrial dynamin-related protein, is mutated in dominant optic atrophy. Nat Genet, 2000. 26(2): p. 207-10.

68. Zuchner, S., et al., Axonal neuropathy with optic atrophy is caused by mutations in mitofusin 2 . Ann Neurol, 2006. 59(2): p. 276-81.

69. Alexander, C., et al., OPA1, encoding a dynamin-related GTPase, is mutated in autosomal dominant optic atrophy linked to chromosome 3q28. Nat Genet, 2000. 26(2): p. 211-5.

70. Yu-Wai-Man, P., et al., Multi-system neurological disease is common in patients with OPA1 mutations. Brain, 2010. 133(Pt 3): p. 771-86.

71. Claramunt, R., et al., Genetics of Charcot-Marie-Tooth disease type 4A: mutations, inheritance, phenotypic variability, and founder effect. J Med Genet, 2005. 42(4): p. 358-65.

72. Waterham, H.R., et al., A lethal defect of mitochondrial and peroxisomal fission. N Engl J Med, 2007. 356(17): p. 1736-41.

73. Chang, C.R., et al., A lethal de novo mutation in the middle domain of the dynamin-related GTPase Drp1 impairs higher order assembly and mitochondrial division. J Biol Chem, 2010. 285(42): p. 32494-503.

74. Reynier, P., et al., OPA3 gene mutations responsible for autosomal dominant optic atrophy and cataract. J Med Genet, 2004. 41(9): p. e110.

75. Baxter, R.V., et al., Ganglioside-induced differentiation-associated protein-1 is mutant in Charcot-MarieTooth disease type 4A/8q21. Nat Genet, 2002. 30(1): p. 21-2. 
76. Anikster, Y., et al., Type III 3-methylglutaconic aciduria (optic atrophy plus syndrome, or Costeff optic atrophy syndrome): identification of the OPA3 gene and its founder mutation in Iraqi Jews. Am J Hum Genet, 2001. 69(6): p. 1218-24.

77. Abrams, A.J., et al., Mutations in SLC25A46, encoding a UGO1-like protein, cause an optic atrophy spectrum disorder. Nat Genet, 2015. 47(8): p. 926-32.

78. Janer, A., et al., SLC25A46 is required for mitochondrial lipid homeostasis and cristae maintenance and is responsible for Leigh syndrome. EMBO Mol Med, 2016. 8(9): p. 1019-38.

79. Wan, J., et al., Loss of function of SLC25A46 causes lethal congenital pontocerebellar hypoplasia. Brain, 2016.

80. Walker, M.A., et al., Powering the immune system: mitochondria in immune function and deficiency. J Immunol Res, 2014. 2014: p. 164309.

81. Pagliarini, D.J., et al., A mitochondrial protein compendium elucidates complex I disease biology. Cell, 2008. 134(1): p. 112-23.

82. Pagliarini, D.J. and J. Rutter, Hallmarks of a new era in mitochondrial biochemistry. Genes Dev, 2013. 27(24): p. 2615-27.

83. Calvo, S.E., K.R. Clauser, and V.K. Mootha, MitoCarta2.0: an updated inventory of mammalian mitochondrial proteins. Nucleic Acids Res, 2016. 44(D1): p. D1251-7.

84. Sanchez-Caballero, L., et al., Mutations in Complex I Assembly Factor TMEM126B Result in Muscle Weakness and Isolated Complex I Deficiency. Am J Hum Genet, 2016. 99(1): p. 208-16.

85. Alston, C.L., et al., Biallelic Mutations in TMEM126B Cause Severe Complex I Deficiency with a Variable Clinical Phenotype. Am J Hum Genet, 2016. 99(1): p. 217-27.

86. Gerards, M., S.C. Sallevelt, and H.J. Smeets, Leigh syndrome: Resolving the clinical and genetic heterogeneity paves the way for treatment options. Mol Genet Metab, 2016. 117(3): p. 300-12.

87. Yarham, J.W., et al., Mitochondrial tRNA mutations and disease. Wiley Interdiscip Rev RNA, 2010. 1(2): p. 304-24.

88. Kaufmann, P., et al., Natural history of MELAS associated with mitochondrial DNA m.3243A>G genotype. Neurology, 2011. 77(22): p. 1965-71.

89. Elo, J.M., et al., Mitochondrial phenylalanyl-tRNA synthetase mutations underlie fatal infantile Alpers encephalopathy. Hum Mol Genet, 2012. 21(20): p. 4521-9.

90. McFarland, R., et al., Multiple neonatal deaths due to a homoplasmic mitochondrial DNA mutation. Nat Genet, 2002. 30(2): p. 145-6.

91. Dimauro, S. and G. Davidzon, Mitochondrial DNA and disease. Ann Med, 2005. 37(3): p. 222-32.

92. Valente, E.M., A. Ferraris, and B. Dallapiccola, Genetic testing for paediatric neurological disorders. Lancet Neurol, 2008. 7(12): p. 1113-26.

93. Santoro, M.R., S.M. Bray, and S.T. Warren, Molecular mechanisms of fragile X syndrome: a twenty-year perspective. Annu Rev Pathol, 2012. 7: p. 219-45.

94. Hagberg, B., et al., A progressive syndrome of autism, dementia, ataxia, and loss of purposeful hand use in girls: Rett's syndrome: report of 35 cases. Ann Neurol, 1983. 14(4): p. 471-9.

95. Barry, R.J., et al., Behavioral aspects of Angelman syndrome: a case control study. Am J Med Genet A, 2005. 132A(1): p. 8-12.

96. Horsthemke, B. and J. Wagstaff, Mechanisms of imprinting of the Prader-Willi/Angelman region. Am J Med Genet A, 2008. 146A(16): p. 2041-52.

97. Metz, G., et al., Rating disease progression of Friedreich's ataxia by the International Cooperative Ataxia Rating Scale: analysis of a 603-patient database. Brain, 2013. 136(Pt 1): p. 259-68.

98. Fahn, S., Concept and classification of dystonia. Adv Neurol, 1988. 50: p. 1-8.

99. Martino, D., M. Stamelou, and K.P. Bhatia, The differential diagnosis of Huntington's disease-like syndromes: 'red flags' for the clinician. J Neurol Neurosurg Psychiatry, 2013. 84(6): p. 650-6.

100. Broussolle, E., et al., Samuel Alexander Kinnier Wilson. Wilson's disease, Queen Square and neurology. Rev Neurol (Paris), 2013. 169(12): p. 927-35.

101. Bird, T.D., Charcot-Marie-Tooth Hereditary Neuropathy Overview, in GeneReviews(R), R.A. Pagon, et al., Editors. 1993: Seattle (WA). 
102. Heatwole, C., et al., Laboratory abnormalities in patients with myotonic dystrophy type 2. Arch Neurol, 2011. 68(9): p. 1180-4.

103. McNally, E.M. and P. Pytel, Muscle diseases: the muscular dystrophies. Annu Rev Pathol, 2007. 2: p. 87109.

104. Wirth, B., An update of the mutation spectrum of the survival motor neuron gene (SMN1) in autosomal recessive spinal muscular atrophy (SMA). Hum Mutat, 2000. 15(3): p. 228-37.

105. Williams, V.C., et al., Neurofibromatosis type 1 revisited. Pediatrics, 2009. 123(1): p. 124-33.

106. Crino, P.B., K.L. Nathanson, and E.P. Henske, The tuberous sclerosis complex. N Engl J Med, 2006. 355(13): p. 1345-56.

107. Maxam, A.M. and W. Gilbert, A new method for sequencing DNA. Proc Natl Acad Sci U S A, 1977. 74(2): p. 560-4.

108. Sanger, F., S. Nicklen, and A.R. Coulson, DNA sequencing with chain-terminating inhibitors. Proc Natl Acad Sci U S A, 1977. 74(12): p. 5463-7.

109. Schloss, J.A., How to get genomes at one ten-thousandth the cost. Nat Biotechnol, 2008. 26(10): p. 11135.

110. Voelkerding, K.V., S.A. Dames, and J.D. Durtschi, Next-generation sequencing: from basic research to diagnostics. Clin Chem, 2009. 55(4): p. 641-58.

111. Goodwin, S., J.D. McPherson, and W.R. McCombie, Coming of age: ten years of next-generation sequencing technologies. Nat Rev Genet, 2016. 17(6): p. 333-51.

112. Li, M., et al., Detecting heteroplasmy from high-throughput sequencing of complete human mitochondrial DNA genomes. Am J Hum Genet, 2010. 87(2): p. 237-49.

113. He, Y., et al., Heteroplasmic mitochondrial DNA mutations in normal and tumour cells. Nature, 2010. 464(7288): p. 610-4.

114. Kukurba, K.R. and S.B. Montgomery, RNA Sequencing and Analysis. Cold Spring Harb Protoc, 2015. 2015(11): p. 951-69.

115. Miller, J.R., S. Koren, and G. Sutton, Assembly algorithms for next-generation sequencing data. Genomics, 2010. 95(6): p. 315-27.

116. Margulies, M., et al., Genome sequencing in microfabricated high-density picolitre reactors. Nature, 2005. 437(7057): p. 376-80.

117. Rasko, D.A., et al., Origins of the E. coli strain causing an outbreak of hemolytic-uremic syndrome in Germany. N Engl J Med, 2011. 365(8): p. 709-17.

118. Schadt, E.E., S. Turner, and A. Kasarskis, A window into third-generation sequencing. Hum Mol Genet, 2010. 19(R2): p. R227-40.

119. Loose, M., S. Malla, and M. Stout, Real-time selective sequencing using nanopore technology. Nat Methods, 2016. 13(9): p. 751-4.

120. Clark, S.J., et al., DNA methylation: bisulphite modification and analysis. Nat Protoc, 2006. 1(5): p. 235364.

121. Eid, J., et al., Real-time DNA sequencing from single polymerase molecules. Science, 2009. 323(5910): p. 133-8.

122. Lange, V., et al., Cost-efficient high-throughput HLA typing by MiSeq amplicon sequencing. BMC Genomics, 2014. 15: p. 63.

123. Fadrosh, D.W., et al., An improved dual-indexing approach for multiplexed $16 \mathrm{~S}$ rRNA gene sequencing on the Illumina MiSeq platform. Microbiome, 2014. 2(1): p. 6.

124. Caporaso, J.G., et al., Ultra-high-throughput microbial community analysis on the Illumina HiSeq and MiSeq platforms. ISME J, 2012. 6(8): p. 1621-4.

125. Bentley, D.R., et al., Accurate whole human genome sequencing using reversible terminator chemistry. Nature, 2008. 456(7218): p. 53-9.

126. Eisenstein, M., The battle for sequencing supremacy. Nat Biotechnol, 2012. 30(11): p. 1023-6.

127. Rhoads, A. and K.F. Au, PacBio Sequencing and Its Applications. Genomics Proteomics Bioinformatics, 2015. 13(5): p. 278-89. 
128. Heather, J.M. and B. Chain, The sequence of sequencers: The history of sequencing DNA. Genomics, 2016. 107(1): p. 1-8.

129. Rehm, H.L., Disease-targeted sequencing: a cornerstone in the clinic. Nat Rev Genet, 2013. 14(4): p. 295300.

130. Botstein, D. and N. Risch, Discovering genotypes underlying human phenotypes: past successes for mendelian disease, future approaches for complex disease. Nat Genet, 2003. 33 Suppl: p. 228-37.

131. Foo, J.N., J.J. Liu, and E.K. Tan, Whole-genome and whole-exome sequencing in neurological diseases. Nat Rev Neurol, 2012. 8(9): p. 508-17.

132. Lupski, J.R., et al., Whole-genome sequencing in a patient with Charcot-Marie-Tooth neuropathy. N Engl J Med, 2010. 362(13): p. 1181-91.

133. Herdewyn, S., et al., Whole-genome sequencing reveals a coding non-pathogenic variant tagging a noncoding pathogenic hexanucleotide repeat expansion in C9orf72 as cause of amyotrophic lateral sclerosis. Hum Mol Genet, 2012. 21(11): p. 2412-9.

134. Bae, B.I., et al., Evolutionarily dynamic alternative splicing of GPR56 regulates regional cerebral cortical patterning. Science, 2014. 343(6172): p. 764-8.

135. Weedon, M.N., et al., Recessive mutations in a distal PTF1A enhancer cause isolated pancreatic agenesis. Nat Genet, 2014. 46(1): p. 61-64.

136. Yang, S., et al., Assessment of circadian function in fibroblasts of patients with bipolar disorder. Mol Psychiatry, 2009. 14(2): p. 143-55.

137. Koopman, W.J., et al., Mitochondrial disorders in children: toward development of small-molecule treatment strategies. EMBO Mol Med, 2016. 8(4): p. 311-27.

138. Takahashi, K. and S. Yamanaka, Induction of pluripotent stem cells from mouse embryonic and adult fibroblast cultures by defined factors. Cell, 2006. 126(4): p. 663-76.

139. Takahashi, K., et al., Induction of pluripotent stem cells from adult human fibroblasts by defined factors. Cell, 2007. 131(5): p. 861-72.

140. Nityanandam, A. and K.K. Baldwin, Advances in reprogramming-based study of neurologic disorders. Stem Cells Dev, 2015. 24(11): p. 1265-83.

141. Krey, J.F., et al., Timothy syndrome is associated with activity-dependent dendritic retraction in rodent and human neurons. Nat Neurosci, 2013. 16(2): p. 201-9.

142. Wen, Z., et al., Synaptic dysregulation in a human iPS cell model of mental disorders. Nature, 2014. 515(7527): p. 414-8.

143. Brennand, K.J., et al., Modelling schizophrenia using human induced pluripotent stem cells. Nature, 2011. 473(7346): p. 221-5.

144. Duffy, J.B., GAL4 system in Drosophila: a fly geneticist's Swiss army knife. Genesis, 2002. 34(1-2): p. 1-15.

145. Matthews, K.A., T.C. Kaufman, and W.M. Gelbart, Research resources for Drosophila: the expanding universe. Nat Rev Genet, 2005. 6(3): p. 179-93.

146. Venken, K.J. and H.J. Bellen, Emerging technologies for gene manipulation in Drosophila melanogaster. Nat Rev Genet, 2005. 6(3): p. 167-78.

147. Dietzl, G., et al., A genome-wide transgenic RNAi library for conditional gene inactivation in Drosophila. Nature, 2007. 448(7150): p. 151-6.

148. Kabashi, E., et al., Zebrafish models for the functional genomics of neurogenetic disorders. Biochim Biophys Acta, 2011. 1812(3): p. 335-45.

149. Volff, J.N., Genome evolution and biodiversity in teleost fish. Heredity (Edinb), 2005. 94(3): p. 280-94.

150. Mouse Genome Sequencing, C., et al., Initial sequencing and comparative analysis of the mouse genome. Nature, 2002. 420(6915): p. 520-62.

151. van der Weyden, L., D.J. Adams, and A. Bradley, Tools for targeted manipulation of the mouse genome. Physiol Genomics, 2002. 11(3): p. 133-64.

152. Kruse, S.E., et al., Mice with mitochondrial complex I deficiency develop a fatal encephalomyopathy. Cell Metab, 2008. 7(4): p. 312-20.

153. Ingraham, C.A., et al., NDUFS4: creation of a mouse model mimicking a Complex I disorder. Mitochondrion, 2009. 9(3): p. 204-10. 


\section{Chapter 1}

154. Timmerman, V., V.E. Clowes, and E. Reid, Overlapping molecular pathological themes link Charcot-MarieTooth neuropathies and hereditary spastic paraplegias. Exp Neurol, 2013. 246: p. 14-25.

155. Zuchner, S. and J.M. Vance, Emerging pathways for hereditary axonopathies. J Mol Med (Berl), 2005. 83(12): p. 935-43.

156. Bargiela, D., et al., Mitochondrial pathology in progressive cerebellar ataxia. Cerebellum Ataxias, 2015. 2: p. 16.

157. Connolly, G.P., Fibroblast models of neurological disorders: fluorescence measurement studies. Trends Pharmacol Sci, 1998. 19(5): p. 171-7. 
Chapter

\section{Pathogenic CWF19L1 variants as a novel cause of autosomal recessive cerebellar ataxia and atrophy}

Minh Nguyen, Iris Boesten, Debby M.E.I. Hellebrekers, Jo Vanoevelen, Rick Kamps, Bart de Koning, Irenaeus F.M. de Coo, Mike Gerards ${ }^{*}$ and Hubert J.M. Smeets ${ }^{*}$

* These authors contributed equally to this work.

Eur J Hum Genet. 2016 Apr;24(4):619-22 


\section{ABSTRACT}

Autosomal recessive cerebellar ataxia (ARCA) is a group of neurological disorders characterized by degeneration or abnormal development of the cerebellum and spinal cord. ARCA is clinically and genetically highly heterogeneous, with over 20 genes involved. Exome sequencing of a girl with ARCA from non-consanguineous Dutch parents revealed two pathogenic variants c.37G>C; p.D13H and c.946A>T; p.K316* in CWF19L1, a gene with an unknown function, recently reported to cause ARCA in a Turkish family. Sanger sequencing showed that the $\mathrm{c} .37 \mathrm{G}>\mathrm{C}$ variant was inherited from the father and the c.946A>T variant from the mother. Pathogenicity was based on the damaging effect on protein function as the c.37G $>C$ variant changed the highly conserved, negatively charged aspartic acid to the positively charged histidine and the c.946A>T variant introduced a premature stop codon. Additionally, 27 patients with ARCA were tested for pathogenic variants in CWF19L1, however, no pathogenic variants were identified. Our data confirms CWF19L1 as a novel but rare gene causing ARCA.

Key words: ARCA, CWF19L1, exome sequencing 


\section{INTRODUCTION}

Autosomal recessive cerebellar ataxia (ARCA) is a group of rare, genetically and clinically heterogeneous neurological disorders affecting both the peripheral and central nervous systems, mainly occurring in children and young adults. ${ }^{1}$ Clinical manifestations range from progressive cerebellar syndromes, as a key feature, to spinocerebellar symptoms with spasticity, ophthalmoplegia, sensory/motor neuropathy, involuntary movements, seizures, cognitive impairment, skeletal abnormalities, and cutaneous disorders. To date, more than 20 genes have been reported to cause ARCA, however, explaining only $40 \%$ of cases. ${ }^{2}$ Recently, pathogenic variants were described in a novel gene (CWF19L1) in two siblings with autosomal recessive ataxia syndrome. ${ }^{3}$ Here, we report a second case, a girl from non-consanguineous Dutch parents, with cerebellar ataxia and atrophy, who was compound heterozygous for pathogenic variants in the CWF19L1 gene. Our results confirm the suggested role of CWF19L1 in rare cases of autosomal recessive cerebellar ataxia.

\section{MATERIALS AND METHODS}

\section{Index patient and 27 additional ARCA patients}

The index patient was born from non-consanguineous parents (Figure 1a) at term after an uncomplicated delivery and had a birth weight of $2480 \mathrm{~g}$. During pregnancy, the echoes of the head showed a height comparable to the $2^{\text {nd }}$ percentile within the Dutch population. Complaints of clumsiness were noted at 5 years of age. Additionally, apart from being a slow learner, there were complaints of mild weakness and fatigability. Neurological investigation at the age of 10 showed a height of $126 \mathrm{~cm}$ (P1), weight of 24 $\mathrm{kg}$ (weight-length P50) and skull circumference of $53 \mathrm{~cm}$ (P50). Furthermore, she showed a slurred, monotonic, sometimes even staccato like, speech and, although she was cooperative, slow in her performance. There was an oculomotor apraxia, no nystagmus and a status after surgical strabismus correction. No retinal pigment layer alterations were noticed and no seizures reported. The patient had normal strength, but a slight dystonic movement disorder in particular in the arms. A mild hypotonia with unsteady stance and broad-based gait was observed as well as intention tremor and overshoot with the finger-nose test. In the tandem walking test, she was more than $5 \mathrm{~cm}$ off, with an increase in unsteadiness over a period of one year. There were no signs of a sensory ataxia or polyneuropathy and no pyramidal tract signs with symmetric and normal reflexes. Family history did not reveal any neurological abnormalities. 
A

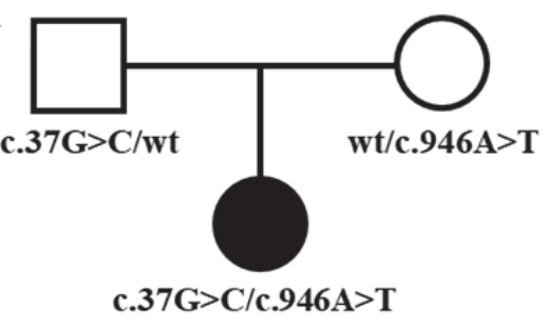

C

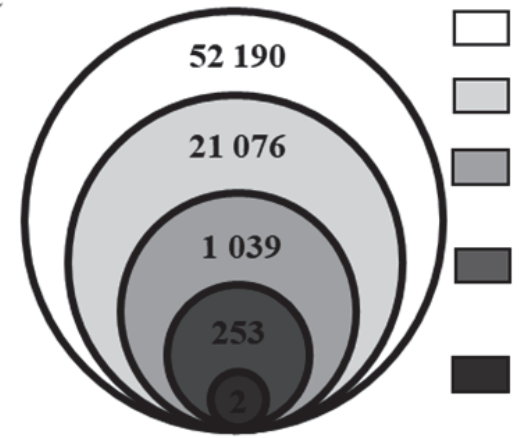

B

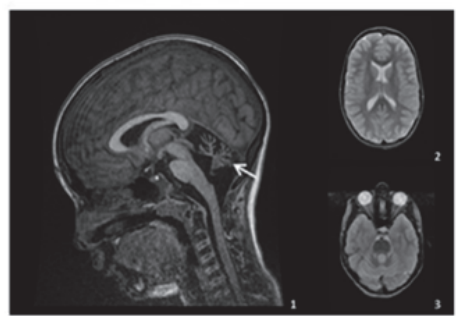

Total number of variants

Exonic and splice site variants

Variants with dbSNP freq $<0.01$

Nonsynonymous variants absent

in in-house database or freq $<0.01$

Candidate genes with 2 or more

variants predicted to be damaging

Figure 1. MRI and exome data of the index patient. A) Pedigree of a Dutch family with autosomal recessive cerebellar ataxia and atrophy. The affected subject is indicated in black. CWF19L1 variants are indicated, illustrating that the $c .37 \mathrm{G}>\mathrm{C}$ is inherited from the father and c.946A>T from the mother. B) The midline sagittal T1-weighted brain magnetic resonance imaging (MRI) demonstrating at the age of 9 years a severe vermian cerebellar hypoplasia with prominent fissures and prominent surrounding cisterns (B-1). Axial T2 weighted $\mathrm{MRI}$ showing a slight aspecific enlargement of the ventricles and cerebellar hypoplasia (B-2 and B-3). C) Schematic representation of the filtering steps of the whole exome data.

Extensive metabolic workup in blood and urine did not reveal any abnormalities. In cerebrospinal fluid, there was an increase detected in myelin basic protein (MBP): 1.2 $\mu \mathrm{g} / \mathrm{l}$ (normal: 0.3-1.0 $\mu \mathrm{g} / \mathrm{l}$ ), whereas concentrations of GFAP, S100 and tau-protein were normal. Magnetic resonance imaging of the brain showed a severe cerebellar hypoplasia, in particular of the vermis (Figure 1b). Compared to 5 years earlier, there was a slight decrease detected in the cerebellar vermis and hemisphere volume.

An additional 27 patients with genetically undiagnosed ARCA were selected based on ataxia as main clinical symptom and confirmed by cerebellar hypoplasia demonstrated by MRI imaging.

\section{Whole exome sequencing and sequence analysis CWF19L1}

After DNA extraction, the exome of the index patient was captured using an exome enrichment kit including the UTR regions (Agilent SureSelect version 4) according to the manufacturers' protocol. Sequencing was performed on an Illumina HiSeq2000 using a 2x100 bp paired end recipe. Basecalling and demultiplexing was done using bcl2fasta 
1.8.4, reads were aligned onto the human reference genome (hg19) using BWA 0.5.9., duplicates marked using the PICARD software suite 1.77 (http://picard.sourceforge.net) , and variants were called using GATK 2.1-8. ${ }^{6,7}$ Annotations were added using an inhouse build annotation database, according to the UCSC RefGene track, dbSNP137 and the dbNSFP (v2.0). ${ }^{8}$ We filtered for genes that contained at least two non-synonymous variants, variants leading to a premature stop codon or splice variants with allele frequencies of $<0.01$ in dbSNP137 and absence in our in-house database of approximately 200 exomes. The variants located in these genes were further evaluated for the impact on protein function by SIFT and Polyphen2 as predicted by the dbNSFP database.

Pathogenic variants identified in CWF19L1 and phenotypes of the index patient were submitted to the gene variant database http://databases.lovd.nl (patient ID 00037591)

For the identification of pathogenic variants in CWF19L1 in additional patients with cerebellar ataxia and atrophy, the exons and flanking introns of CWF19L1 were amplified with specific intronic primers (sequences available on request).

\section{RNA extraction and real- time PCR}

Human fibroblasts were cultured in Dulbecco modified Eagle (DMEM) supplemented with $10 \%$ fetal bovine serum (FBS), 1\% L-glutamine, and $1 \%$ penicillin/streptomycin (Gibco BRL) and incubated in humidified atmosphere of $5 \% \mathrm{CO}_{2}$ at $37^{\circ} \mathrm{C}$. Total RNA was extracted with TRIzol (Invitrogen) and converted into cDNA using RT First Strand Kit (Qiagen). Expression of CWF19L1 was determined using SYBR Green qPCR on a 7900HT RT-PCR System (Life Technologies). TBP was used to normalize expression levels.

To test for nonsense mediated decay, PCR amplification of CDNA with primers designed to flank the variant introducing the premature stop codon, is followed by Sanger sequencing.

\section{RESULTS}

Exome sequencing was performed in a patient with ARCA, from Dutch nonconsanguineous parents, to identify the underlying genetic defect (Figure 1). In total, 52,190 variants were detected. After removing intergenic, UTR-variants, non-splice site related intronic variants, synonymous variants, variants with an allele frequency $>0.01$ in dbSNP137 and variants present in our in-house database, only 253 variants were left. Seven genes harbored $\geq 2$ variants of which only two (CWF19L1 and MYO18B) harbored two variants predicted to be damaging according to SIFT (score $<0.05)$ and Polyphen2 (score $>0.85$ ) or resulted in a premature stop codon. MYO18B is unlikely to cause ARCA as variants in this gene are associated with lung cancer ${ }^{9}$ or linked to Klippel-Feil anomaly and myopathy ${ }^{10}$, which were not present in our patient. However, during the course of 
our studies, CWF19L1 was reported as a novel cause of $A R C A^{3}$ and we focused further on this gene. The CWF19L1 c.37G $>C$ ( $r .37 G>C$ ) variant results in the substitution of the highly conserved negatively charged aspartic acid to the positively charged histidine at amino acid position 13 (Figure 2a) whereas the c.946A>T (r.946A>U) substitution results in a premature stop codon at amino acid position 316, (NM_018294.4) (Figure 2a).

(A)

c.964+1G>A (Burns et al, 2014)

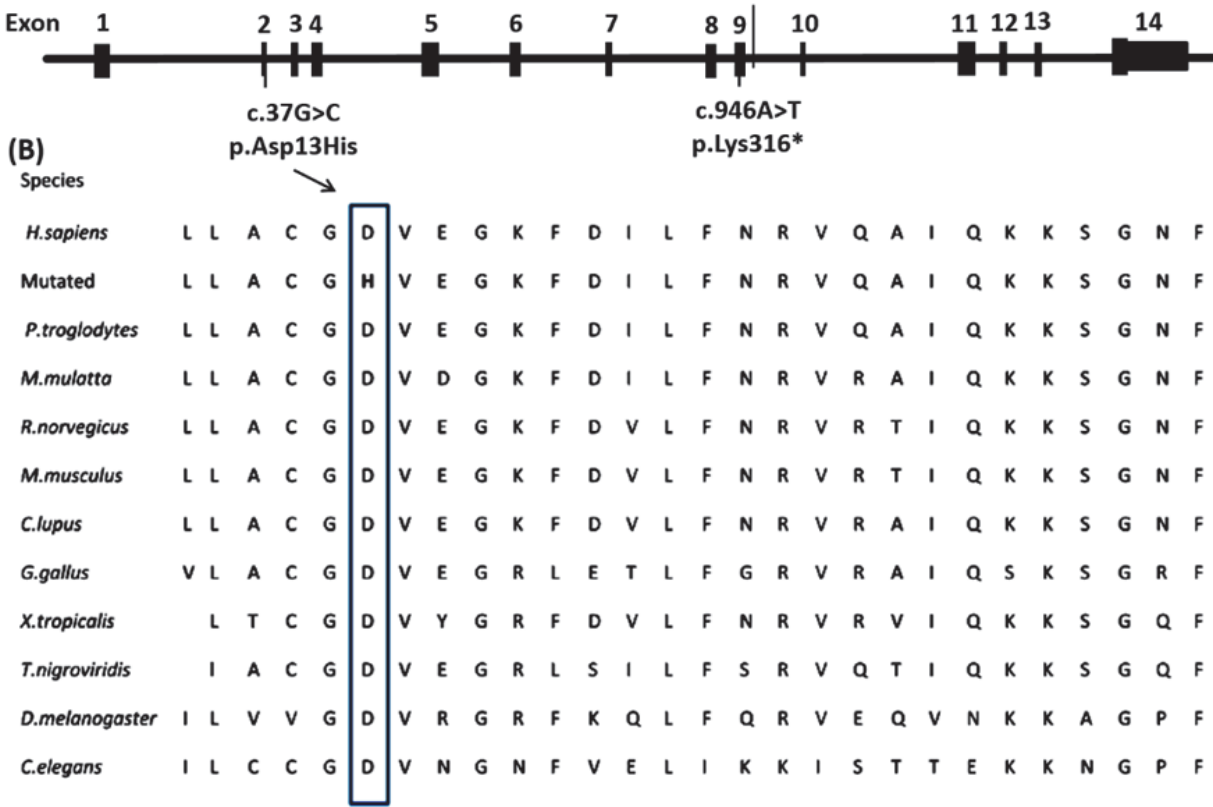

Figure 2. A) Schematic representation of CWF19L1 and positions of variants. B) Conservation of affected amino acid residue of p.Asp13His from H. sapiens to C. elegans (affected amino acid shown in bold.)

Sanger sequencing showed that the father was heterozygous for the $\mathrm{C.37G}>\mathrm{C}$ variant and the mother for c.946A>T variant, confirming that the patient was compound heterozygous (Figure 1a).

CWF19L1 expression level was normal in patient's fibroblasts compared to control (Figure 3a). Sequence analysis of cDNA from patient's fibroblasts showed a decreased amount of the r.946A $>U$ transcript compared to the r.37G $>C$ (Figure 3b), which was not seen on DNA level, indicating this allele is degraded by nonsense mediated mRNA decay. 
$\mathbf{A}$

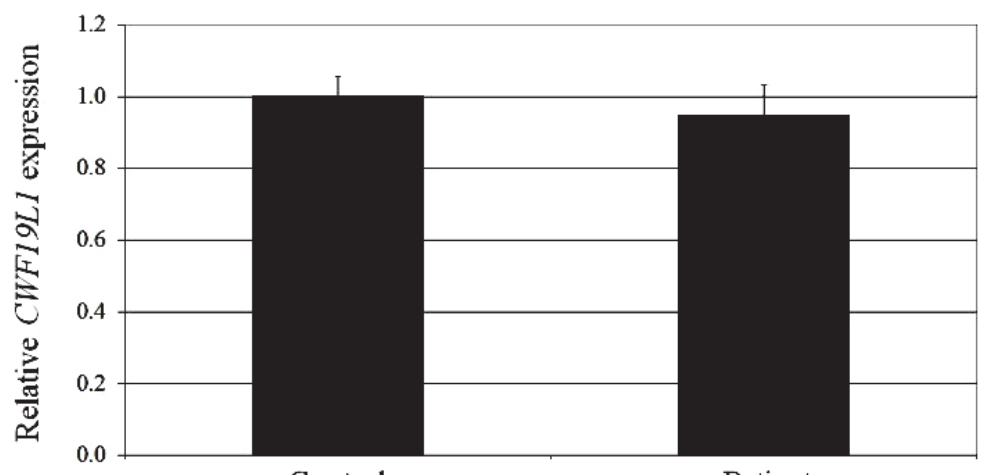

Control

Patient

B
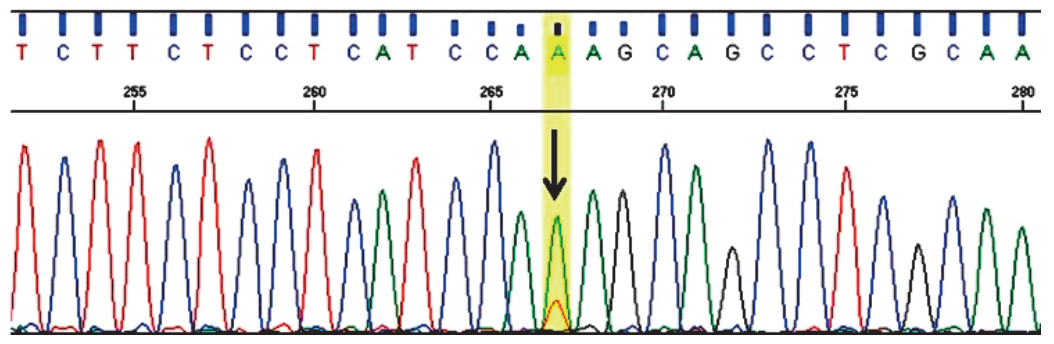

Figure 3. CWF19L1 mRNA expression and maturation. (A): CWF19L1 expression in patient's fibroblasts. Error bars indicate standard deviation. (B): Sequence of CWF19L1 cDNA product shows decreased amount of the c.946A > T transcript compared to the $\mathrm{c} .37 \mathrm{G}>\mathrm{C}$ transcript.

Subsequently, 27 additional patients with autosomal recessive cerebellar ataxia and atrophy were selected and screened for variants in CWF19L1. However, no pathogenic variants were identified in CWF19L1 for these patients.

\section{DISCUSSION}

Using whole exome sequencing we identified two novel heterozygous variants in CWF19L1 causing ARCA in a Dutch non-consanguineous family. One variant p.D13H changes the highly conserved, negatively charged aspartic acid into the positively charged histidine and is predicted to affect protein stability or function. The other variant leads to a premature stop codon p.K316* which results in nonsense mediated mRNA decay. Total mRNA of the patient's fibroblasts was expressed at level similar to the control indicating that increased c.37G $>C$ transcript compensated the degradation by nonsense mediated decay.

During the course of our studies, CWF19L1 was recently suggested as a novel ataxia gene in a Turkish family with a comparable phenotype to our patient. ${ }^{3}$ The authors 
showed that knock down of the homologue of CWF19L1 in zebrafish results in cerebellar and movement abnormalities and that the encoded protein C19L1 is expressed throughout the brain suggesting an important role for C19L1 in neural development. ${ }^{3}$ Although the exact function of the C19L1 is still unknown, the nuclear localization in combination with a metallophosphatase domain, which is found in RNA lariat debranching enzymes, suggests a role in mRNA processing. ${ }^{3}$ However, further studies are needed to determine the exact function of this protein and its role in brain development. Detailed functional characterization of C19L1 will provide more information on different causes of ARCA and possibly reveal novel candidates for analysis of pathogenic variants in patients and/or targets for therapy. Screening of CWF19L1 for pathogenic variants in an additional 27 genetically undiagnosed patients with ARCA did not reveal any additional pathogenic variants, indicating variants in CWF19L1 are a rare cause of ARCA.

In conclusion, our report confirms the recent study on pathogenic variants in CWF19L1 as a possible cause of ARCA, adding CWF19L1 to the already long list of candidate genes for patients with ARCA.

\section{CONFLICT OF INTEREST}

The authors declare no conflict of interest.

\section{FUNDING}

The work is supported by the Alma in Silico project, which is financed by the Interreg IV European funds, The Walloon Region, The North Rhine Westphalia, The Flemish Community, The Belgian Province of Limburg and The Dutch Province of Limburg, as well as by the Universities of Maastricht and Liège, by the Prinses Beatrix Spierfonds (grant W.OR11-24) and the Stichting Metakids. 


\section{REFERENCES}

1. Anheim M, Tranchant C, Koenig M: The autosomal recessive cerebellar ataxias. N Engl J Med 2012; 366: 636-646.

2. Sailer A, Houlden $\mathrm{H}$ : Recent advances in the genetics of cerebellar ataxias. Curr Neurol Neurosci Rep 2012; 12: 227-236.

3. Burns R, Majczenko K, Xu J et al: Homozygous splice mutation in CWF19L1 in a Turkish family with recessive ataxia syndrome. Neurology 2014; 83: 2175-2182.

4. Li H, Durbin R: Fast and accurate short read alignment with Burrows-Wheeler transform. Bioinformatics 2009; 25: 1754-1760.

5. Li H, Durbin R: Fast and accurate long-read alignment with Burrows-Wheeler transform. Bioinformatics 2010; 26: 589-595.

6. McKenna A, Hanna M, Banks E et al: The genome analysis toolkit: a MapReduce framework for analyzing next-generation DNA sequencing data. Genome Res 2010; 20: 1297-1303.

7. DePristo MA, Banks E, Poplin R et al: A framework for variation discovery and genotyping using nextgeneration DNA sequencing data. Nat Genet 2011; 43: 491-498.

8. Liu X, Jian X, and Boerwinkle E: dbNSFP v2.0: a database of human non-synonymous SNVs and their functional predictions and annotations. Hum Mutat 2013; 34: E2393-E2402.

9. Nishioka M, Kohno T, Tani M: MYO18B, a candidate tumor suppressor gene at chromosome 22q12.1, deleted, mutated, and methylated in human lung cancer. Proc Natl Acad Sci USA 2002; 99: 12269-74.

10. Alazami AM, Kentab AY, Fageih E et al: A novel syndrome of Klippel-Feil anomaly, myopathy, and characteristic facies is linked to a null mutation in MYO18B. J Med Genet 2015; 0: 1-5. 



\section{Chapter}

\section{Novel pathogenic SLC25A46 splice-site mutation causes an optic atrophy spectrum disorder}

Nguyen M, Boesten I, Hellebrekers DMEI, Mulder-den Hartog NM, de Coo IFM, Smeets HJM $^{*}$, Gerards M*

* These authors contributed equally to this work.

Clin Genet. 2017 Jan;91(1):121-125 


\section{ABSTRACT}

The inherited optic neuropathies comprise a group of genetically heterogeneous disorders causing optic nerve dysfunction. In some cases, optic neuropathies are associated with cerebellar atrophy which mainly affects the vermis. Here, we describe a Moroccan girl of consanguineous parents with optic atrophy and cerebellar atrophy. Exome sequencing revealed a novel homozygous mutation (c.283+3G>T) in the donor splice site for exon 1 of SLC25A46. RNA analysis revealed that an alternative splice site within exon 1 was used leading to a premature termination codon within exon 2. SLC25A46 mRNA expression showed there is no wild-type transcript present in the patient and the mutant transcript does not undergo nonsense mediated mRNA decay. Furthermore, we observed c.283+3G>T SLC25A46 mutation induces mitochondrial fragmentation. An additional 10 patients with optic atrophy and cerebellar atrophy, which were negative for mtDNA and OPA1 variants, were tested for pathogenic mutations in the SLC25A46 gene. However, no additional variants were identified. Our findings confirm the recent report of pathogenic SLC25A46 mutations as a novel cause for optic atrophy spectrum disorder.

Key words: optic atrophy, SLC25A46, cerebellar atrophy, exome sequencing, mitochondrial dynamics 


\section{INTRODUCTION}

Inherited optic neuropathies are genetically heterogeneous, characterized by a decrease in visual acuity and optic atrophy as the main clinical phenotype. The most frequent inherited optic neuropathies are autosomal dominant optic atrophy and Leber's hereditary optic neuropathy (LHON). Optic neuropathies may be inherited in an autosomal dominant, autosomal recessive, X-linked or maternal fashion. Dominant optic atrophy is mainly caused by pathogenic OPA1 mutations, involved in the fusion of the inner mitochondrial membrane. Pathogenic OPA1 mutations also cause an optic atrophy 'plus' phenotype in which patients present with additional neurological phenotypes including cerebellar atrophy $(1,2)$. In contrast, OPA3 is involved in mitochondrial fission, although the exact role of OPA3 in fission still needs to be determined. Pathogenic variants in OPA3 have been shown to cause recessive optic atrophy (3). X-linked optic atrophy has been linked to OPA2 locus on the $X$ chromosome, but no causative gene defect has been identified so far (4). LHON, a maternally inherited optic neuropathy, is predominantly caused by point mutations in the mitochondrial DNA affecting different subunits of complex I (2).

Recently, a paper was published, reporting on SLC25A46 mutations as the cause of optic atrophy and axonal Charcot Marie Tooth and cerebellar atrophy (5). In our study, we identified a novel pathogenic splice site SLC25A46 mutation, in a family with optic atrophy and cerebellar atrophy confirming the expanding spectrum of genes involved in these disorders.

\section{MATERIALS AND METHODS}

\section{Whole exome sequencing and sequence analysis SLC25A46}

Whole exome sequencing was performed as previously described (6). For variant analysis of SLC25A46 in additional patients with optic atrophy and cerebellar atrophy, the exons and flanking introns of SLC25A46 were amplified with specific intronic primers (Supplementary Table 1). The effect of splice-site mutations was predicted based in the algorithms integrated in the Alamut software version 2.4 (SpliceSiteFinder-like, MaxEntScan, NNSplice, GeneSplicer, and Human Splicing Finder).

\section{SLC25A46 mRNA analyses}

Human fibroblasts were cultured in Dulbecco modified Eagle (DMEM) supplemented with $10 \%$ fetal bovine serum (FBS), 1\% L-glutamine, and 1\% penicillin/streptomycin (Gibco BRL) and incubated in humidified atmosphere of $5 \% \mathrm{CO}_{2}$ at $37^{\circ} \mathrm{C}$. To determine 
SLC25A46 expression, total RNA was isolated in triplicate from cultured fibroblasts using phenol-chloroform extraction and ethanol precipitation. Subsequently, cDNA was synthesized using the RT First Strand Kit with oligo (dT) primers (Qiagen). To evaluate the effect of the SLC25A46 mutation on splicing, RT- PCR products spanning exon1-exon3 were analyzed using specific primers (Supplementary Table 1). Specific primers were designed in exon 8 for quantification of SLC25A46 transcript levels and for TBP as the reference gene for normalization (Supplementary Table 1). To detect the amount of wild-type SLC25A46 mRNA in the patient, we designed a forward primer located within the region which was deleted due to alternative splicing and the reverse primer outside this deleted region (Supplementary Table 1).

To inhibit the nonsense mediated mRNA decay, fibroblasts were treated with $300 \mu \mathrm{g}$ puromycin/ml culture medium for 6 hours as previously described (7). Quantification was performed by real-time quantitative PCR on the 7900HT Fast Real-Time PCR System (Life Technologies) using the SYBR Green qPCR kit. Results presented are the mean of three independent experiments. Statistical significance was assessed by a standard unpaired, two- tailed Student's t-test. P value under 0.05 was considered significant.

\section{Live cell imaging and quantification of mitochondrial morphology}

$2 \times 10^{4}$ fibroblasts were seeded in 12 - well plate (Microtech) and left to adhere overnight. Mitochondrial network morphology was assessed after cell staining with $200 \mathrm{nM}$ Mitotracker Green FM (Invitrogen) for $45 \mathrm{~min}$ at $37^{\circ} \mathrm{C}, 5 \% \mathrm{CO}_{2}$. The fibroblasts were washed twice in PBS and visualized on a Leica confocal microscope. Images were captured with the Leica Microsystems LAS AF Software. Quantification of the mitochondrial morphology was performed using a previously described ImageJ macro (8).

\section{RESULTS}

Index patient and 10 additional optic atrophy and cerebellar atrophy patients

The index patient was the first child of Moroccan consanguineous parents. After a pregnancy complicated by polyhydramnios, an asphyxiated girl was born at term (apgar score 6 and 6 after 10 and 20 minutes). She required 30 hours postpartum assisted ventilation because of central respiratory insufficiency. The girl presented clubfoot posture, hypotonia and double sided optic atrophy. From birth onwards, myoclonic jerks were seen which were more present over the left than the right side of the body. No myotatic reflexes could be elicited. The patient died because of respiratory deficiency after 7 days. She has three younger healthy sisters and one brother. The youngest sister suffered at birth from a hypertrophic cardiomyopathy. However, the cardiomyo- 
pathy resolved in the first year of life spontaneously. Family history revealed a brother of the mother with a son who died at three days of age for unknown reasons.

An elevated lactic acid in CSF (3.78, normal: $0.9-2.8 \mathrm{mmol} / \mathrm{l}$ ) was the only abnormality detected in the extensive metabolic work up of blood plasma, urine and CSF. Serological tests did not show any signs for congenital infections. Lactate to pyruvate ratio was elevated in fibroblasts $(70$, normal range $22 \pm 15)$ and a decrease in all individual complexes with a disproportionate decrease of cytochrome c oxidase activity was found in fibroblasts and vastus lateralis muscle. The magnetic resonance imaging of the brain revealed atrophic cerebellar hemispheres with a less severe affected vermis and a retrocerebellar arachnoid cyst. There was diffuse central and peripheral atrophy of the brain with slight enlargement of the ventricular system (Supplementary Fig. 1B). Flair imaging showed slight periventricular areas of gliosis. A delayed background pattern without epileptic discharges was seen on EEG. The differential diagnosis strongly pointed to a progressive mitochondrial disorder. The optic atrophy and the diffuse cerebral atrophy with severe cerebellar atrophy could not rule out olivopontocerebellar or cerebro-ocular-muscular syndromes caused by other disease mechanisms.

Ten additional patients were selected for screening of mutations in SLC25A46 upon their opticopathy and/or cerebellar atrophy (cerebellar hemisferes and/or vermis atrophy) without a genetic diagnosis. MtDNA and OPA1 mutations were excluded.

\section{Exome sequencing and data analysis}

Exome sequencing was performed in the index patient (Supplementary Fig. 1A). A total of 61,303 variants were detected, but after filtering on homozygosity, conservation, frequency and pathogenicity prediction (Supplementary Fig. 1C) only two different candidate genes remained (SLC25A46 and CACNA1G). The SLC25A46 variant was most promising as mutations in this gene have been reported to cause optic atrophy, which is a key clinical symptom in our patient (5). The variants in CACNA1G (c.4353+3 G>A; NM_198383.2) was unlikely to be causative, as CACNA1G is a calcium channel important for calcium-dependent processes with no phenotypic link to our patient. The variant detected in SLC25A46 (c. 283+3 G>T; NM_138773.2) is a novel variant in intron 1 which is predicted to affect splicing according to the MaxEnt Scan algorithm (-84.9\%), and NNSplice (-77.4\%).

To confirm the splicing effect, PCR primers were designed in exon 1 and 3 (Fig. 1A). The cDNA product, derived from fibroblast RNA of the patient, was only $172 \mathrm{bp}$ in size, whereas the product in case of normal splicing is $363 \mathrm{bp}$ (Fig. 1B). No normal cDNA was observed in the patient indicating almost no normal splicing, leading to a smaller mRNA. Sequence analysis revealed a deletion of 191 base pairs of exon 1 in the cDNA of the patient, which results in a frameshift and a premature translation termination signal in exon 2 (Fig. 1C, 1D). SLC25A46 RNA expression was 35\% in patients' fibroblasts compared to controls (Fig. 1E). Quantification of the wild-type transcript using wild-type 
specific qPCR primers, showed that only 1\% of wild-type SLC25A46 mRNA was present in patient fibroblasts (Fig. 1E). To investigate whether the decreased expression of mutant transcript was due to nonsense mediated mRNA decay (NMD), patient cells were treated with puromycin, a NMD inhibitor. No change in SLC25A46 transcript level was detected upon treatment with puromycin compared to untreated cells (Fig. 1F) indicating the transcript escapes NMD despite the introduction of a premature termination codon (PTC). Sanger sequencing of the family provided additional evidence by showing that the father, mother and a healthy sister were heterozygous for the mutation. Sequence analysis of SLC25A46 in ten additional patients with optic atrophy and cerebellar atrophy did not reveal additional pathogenic mutations.

(A)

Forward primer

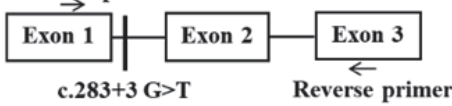

(C)

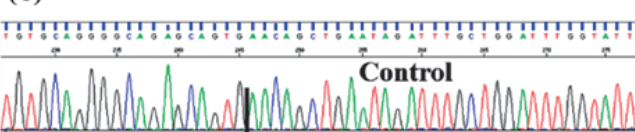

Exon 1 Exon 2

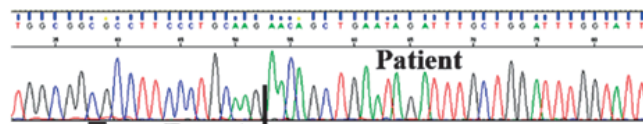

(E)

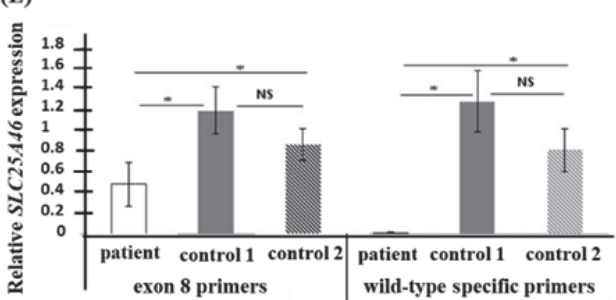

(B)

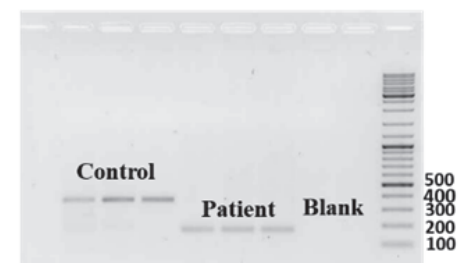

(D)

Donor splice site

GTGैGTG

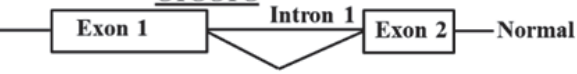

Cryptic splice site Splicing of mRNA

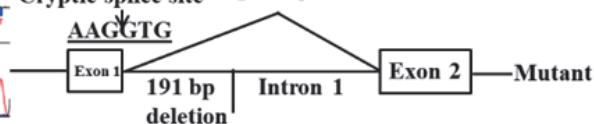

(F)

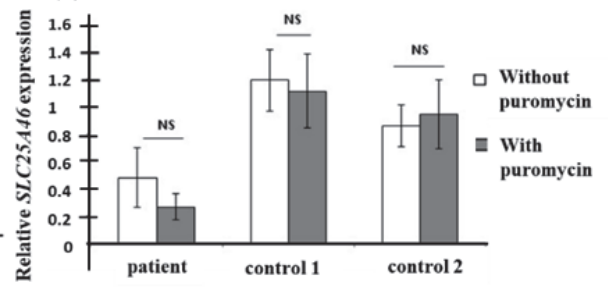

Fig. 1: The effect of the c.283+3G>T splice site mutation in SLC25A46 and relative SLC25A46 mRNA expression (A) The position of primers used to amplify spliced mRNA product. (B) RT-PCR analysis of SLC25A46 covering exon1-3 of mRNA extracted from fibroblasts of the patient and a control. (C) Sequence of RT-PCR products demonstrating alternative a deletion of 191 nucleotides in exon 1 of the patient, causing a frameshift and premature stop codon in exon 2. (D) Schematic representation of the transcripts resulting from incorrect splicing. (E) SLC25A46 mRNA expression in fibroblasts of the patient and two controls using different primer pairs specific for non-deleted and deleted exon 1 region. (F) Upon puromycin treatment for 6 hours, SLC25A46 mRNA expression did not increase in fibroblasts of the patient and two controls. Experiments were performed in triplicate. Error bars indicate standard deviation. ${ }^{*} p<0.05$ 


\section{SLC25A46 mutation induces mitochondrial fragmentation}

Since SLC25A46 was reported to be involved in mitochondrial dynamics, the mitochondrial network was studied in patient's fibroblasts using Mitotracker. In control cells, mitochondria exhibited a balanced mitochondrial network between the fragmented and filamentous states while in fibroblasts from the patient, increased fragmentation of the mitochondrial network was observed (Fig. 2), indicating an important role of SLC25A46 in mitochondrial dynamics.
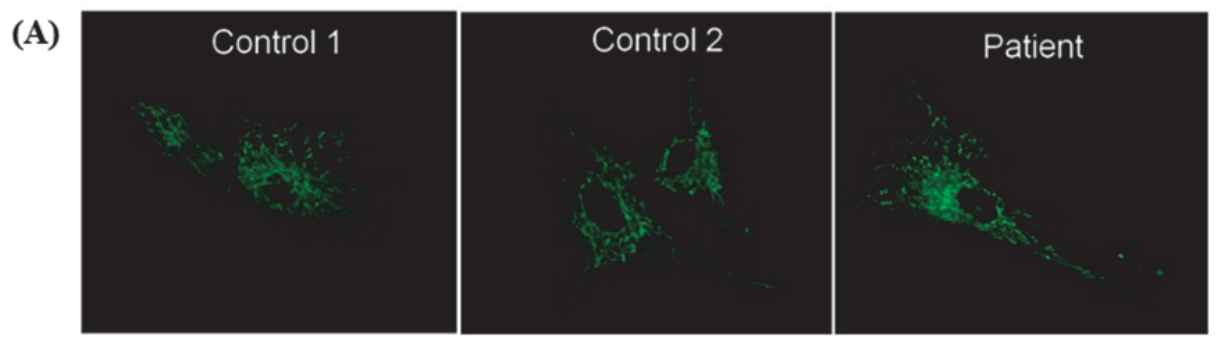

(B)

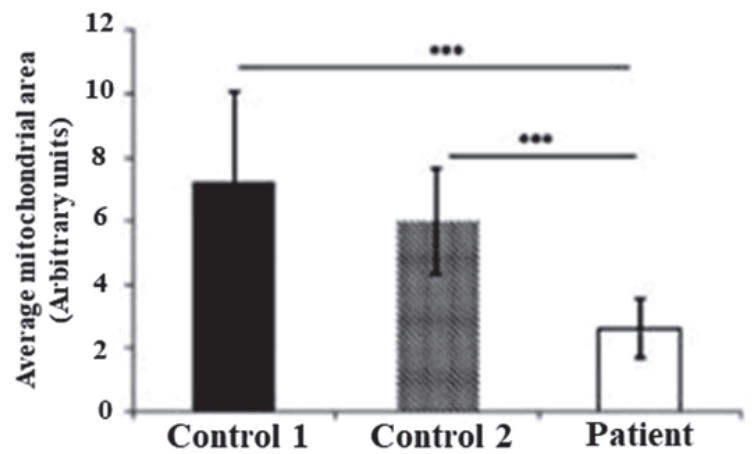

Fig. 2: Effect of the SLC25A46 c.283+3G>T mutation on mitochondrial network morphology (A) Representative confocal images of fibroblasts stained with Mitotracker Green FM (Invitrogen) to identify mitochondrial network. The patient fibroblasts revealed fragmented mitochondrial network compared two controls. (B) Quantification of the average area of mitochondria (*** $p<0.001)$

\section{DISCUSSION}

Inherited optic atrophies are rare diseases affecting the survival and function of the optic nerve and mainly caused by pathogenic mutations in the OPA1 gene, which is involved in mitochondrial fusion. Some patients manifest with additional neurological symptoms. However, OPA1 mutations explain $60-70 \%$ of the patients with autosomal dominant optic atrophy indicating the involvement of additional genes (1). Exome sequencing in a girl with optical nerve and cerebellar atrophy revealed a novel homozy- 
gous splice site mutation in the SLC25A46 gene. The mutation completely abolishes the donor splice site within intron 1, leading to a frameshift and a premature termination in exon 2. As a result, no functional SLC25A46 is present. Interestingly, the mutant transcript escaped NMD, probably due to the location of the PTC at the beginning of exon 2. It is possible that another start site downstream of the PTC is used. However, the first start site downstream of the PTC is out of frame and would lead to a different protein. Therefore, it is highly unlikely any functional or truncated form of SLC25A46 is present, yet we cannot fully exclude an aberrant form of SLC25A46 is present. Only one other, recent report describes SLC25A46 mutations in patients with clinical symptoms of optic atrophy and cerebellar atrophy, comparable to those found in our patient (Table 1). One of the patients reported by Abrams et al shows a similar age of onset and severe phenotype. This patient born to American parents carries one frame shift mutation and one amino acid substitution in the mitochondrial carrier domain (5). It is possible that the amino acid substitution disrupts the mitochondrial carrier domain, essential for SLC25A46 function. This may explain the overlapping severe phenotypes as our patient also has no functional SLC25A46. 
Novel pathogenic SLC25A46 splice-site mutation causes an optic atrophy spectrum disorder

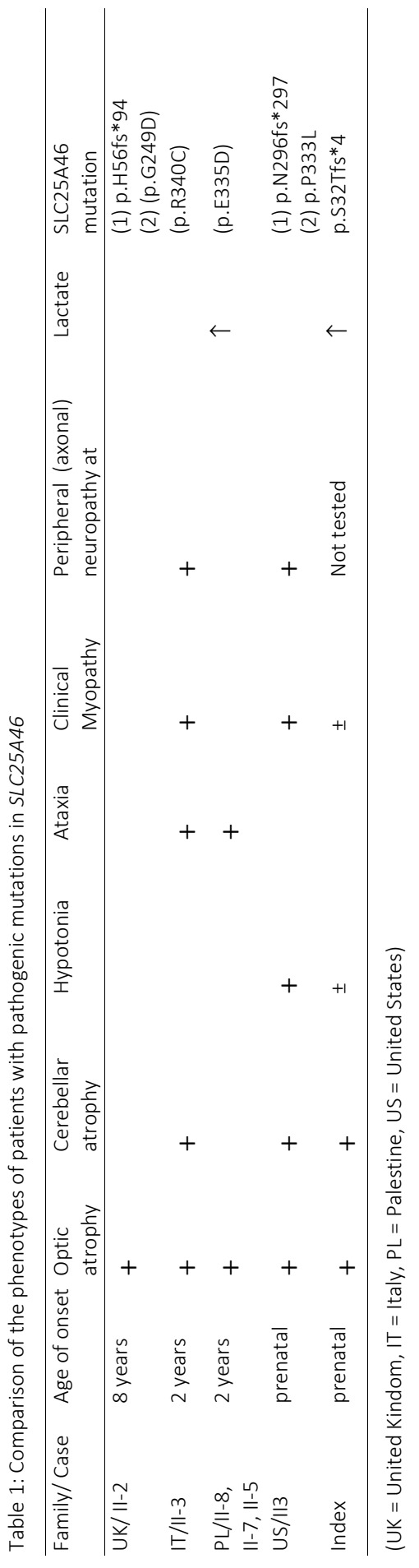


Screening of SLC25A46 in 10 additional patients with optic atrophy and cerebellar atrophy (cerebellar hemisferes and/or vermis atrophy), which were negative for mtDNA and OPA1 variants, did not reveal any additional pathogenic mutations, indicating SLC25A46 are a rare cause of the patient with cerebellar and optic atrophy. These patients may carry pathogenic variants in other, yet to discover, genes involved in mitochondrial dynamics.

The SLC25 gene family is the largest family of solute carriers known, covered by 53 nuclear genes. These carriers are mainly embedded in the inner mitochondrial membrane and have a similar function despite a broad variety in sizes and nature of their substrates (9). Unlike the majority of SLC25 family members, SLC25A46, a homolog of the $S$. cerevisiae Ugo1, is recruited to the outer membrane of mitochondria and interacts with mitofilin (5). siRNA-mediated knock down of SLC25A46 in cultured cells and in zebrafish increased mitochondrial connectivity and caused severe effects on the neuronal development and maintenance in zebrafish (5). Our study did not show an increase in mitochondrial branching in patient- derived fibroblasts compared to controls. In contrast, fibroblasts of our patient showed a fragmented mitochondrial network. This may be explained by the fact that in knock down experiments there is still some protein left while our patients' fibroblasts do not contain any SLC25A46. The decreased OXPHOS activities observed in our patient might be the result of the inability of eliminating damaged mitochondria from the mitochondrial network. Although further studies are needed, the similarity in phenotypes caused by pathogenic mutations in OPA1, MFN2 and SLC25A46 suggest common pathogenic mechanisms (5).

In conclusion, our report confirms the recent study on pathogenic mutations in SLC25A46 as a novel cause of optic atrophy and cerebellar atrophy, which is important for diagnosis and for the development of novel treatments. Based on these results SLC25A46 should be added to the list of genes screened for mutations in patients with optic atrophy 'plus' phenotypes.

\section{CONFLICT OF INTEREST}

The authors declare no conflict of interest.

\section{FUNDING}

The work is supported by the Alma in Silico project, which is financed by the Interreg IV European funds, The Walloon Region, The North Rhine Westphalia, The Flemish Community, The Belgian Province of Limburg and The Dutch Province of Limburg, as well as by the Universities of Maastricht and Liège, by the Prinses Beatrix Spierfonds (grant 
Novel pathogenic SLC25A46 splice-site mutation causes an optic atrophy spectrum

disorder

W.OR11-24) and the Stichting Metakids. Mrs N. Mulder-Den Hartog was supported by the patient organization fund join4energy. 


\section{REFERENCES}

1. Amati-Bonneau P, Valentino ML, Reynier P et al. OPA1 mutations induce mitochondrial DNA instability and optic 'plus' phenotypes. Brain 2008: 131: 338-51.

2. Yu-Wai-Man P, Griffiths P, Hudson G et al. Inherited mitochondrial optic neuropathies. J. Med. Genet 2009: 46: 145-158.

3. Ryu SW, Jeong HJ, Choi M et al. Optic atrophy 3 as a protein of the mitochondrial outer membranes induces mitochondrial fragmentation. Cell Mol Life Sci 2010: 67: 2839-50.

4. Katz BJ, Zhao Y, Warner JE et al. A family with X-linked optic atrophy linked to the OPA2 locus Xp11.4-Xp11.2. Am J Med Genet 2006: 140: 2207-2211.

5. Abrams AJ, Hufnagel RB, Rebelo A et al. Mutations in SLC25A46, encoding a UGO1-like protein, cause an optic atrophy spectrum disorder. Nat Genet 2015: 47: 926-32.

6. Nguyen M, Boesten I, Hellebrekers D et al. Pathogenic CWF19L1 variants as a novel cause of autosomal recessive cerebellar ataxia and atrophy. Eur J Hum Genet 2015.

7. Gerards M, van den Bosch B, Calis C et al. Nonsense mutations in CABC1/ADCK3 cause progressive cerebellar ataxia and atrophy. Mitochondrion 2010: 10: 510-5

8. Dagda RK, Cherra SI III, Kulich SM et al. Loss of PINK1 function promotes mitophagy through effects on oxidative stress and mitochondrial fission. J Biol Chem. 2009: 284: 13843-55.

9. Palmieri F. The mitochondrial transporter family SLC25: identification, properties and physiopathology. Mol Aspects Med 2013: 34: 465-84. 
(A)

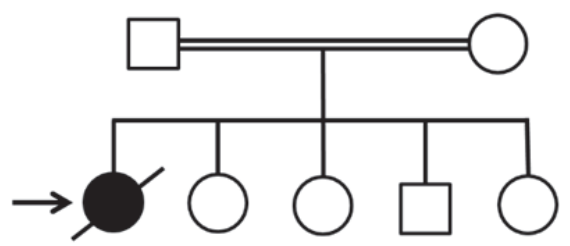

(C)

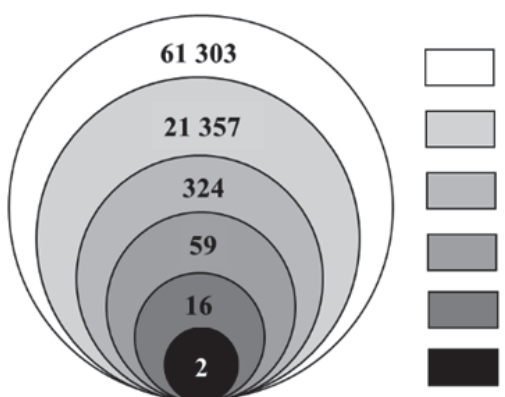

(B)

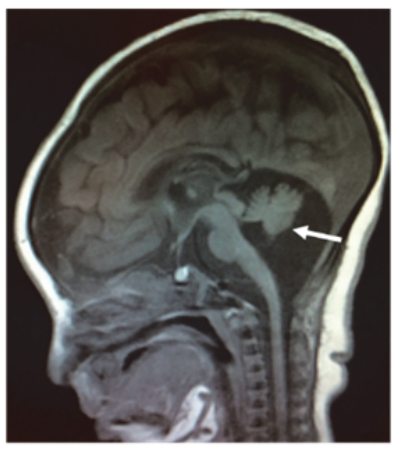

Total number of variants

\section{Homozygous variants}

Variants with freq $<0.01$ or in-house freq $<0.01$

Exonic and splice- site variants

Variants predicted to be damaging

Variants in homozygosity mapping

Fig. S1. Magnetic resonance imaging (MRI) and exome data of the index patient. (a) Pedigree of a consanguineous family with optic atrophy and cerebellar atrophy. The index patient is indicated in black. All other family members are healthy. (b) Sagittal T1 weighted brain MRI shows hypoplastic cerebellum, small brainstem and hypoplastic corpus callosum. (c) Schematic representation of the filtering steps of the exome data. 
Chapter 3

Supplementary Table 1: Primers sequences (Forward [F] and reverse [R])

\begin{tabular}{|c|c|c|c|}
\hline Gene-Exon & Primer sequence $5^{\prime}-3^{\prime}$ & Application & $\begin{array}{l}\text { Fragment size } \\
\text { (bp) }\end{array}$ \\
\hline$S L C 25 A 46-1 A$ & $\begin{array}{l}\text { F: AACGTGACTTCCGGTTG } \\
\text { R: CCTTCGTACGGGGTGGA }\end{array}$ & sequencing & 392 \\
\hline SLC25A46-1B & $\begin{array}{l}\text { F: CCACTGGGTGACGACTC } \\
\text { R: AGGGGCAGAGGACCAAA }\end{array}$ & sequencing & 355 \\
\hline SLC25A46-2 & $\begin{array}{l}\text { F: AGGTTTAAATGAACAGCAGGTTAAT } \\
\text { R: CAAAGAAACAACCTAAAGGAAGC }\end{array}$ & sequencing & 312 \\
\hline$S L C 25 A 46-3$ & $\begin{array}{l}\text { F: TTCAGGCAAGAATAATTTCAGAAAG } \\
\text { R: TCATTTATGTGAGTTCTGCCC }\end{array}$ & sequencing & 258 \\
\hline$S L C 25 A 46-4$ & $\begin{array}{l}\text { F: TTCCACCATATTTCTTTCATGGC } \\
\text { R: CCAAACTGAAAGTAATTATTCTTGC }\end{array}$ & sequencing & 287 \\
\hline$S L C 25 A 46-5$ & $\begin{array}{l}\text { F: GGAAAATGTCTACACAATATCAAGT } \\
\text { R: ATGTACCGCCTGCATCTA }\end{array}$ & sequencing & 299 \\
\hline$S L C 25 A 46-6$ & $\begin{array}{l}\text { F: AAAATTGGGCTAATTCCAGATG } \\
\text { R: CTGCTCTAATTTCCTTCTCTACAG }\end{array}$ & sequencing & 273 \\
\hline$S L C 25 A 46-7$ & $\begin{array}{l}\text { F: AGCTTGACTATAAATTATGTTGACA } \\
\text { R: AACGTGCGATATAACTGTGAT }\end{array}$ & sequencing & 346 \\
\hline SLC25A46-8A & $\begin{array}{l}\text { F: CCTCTTCAGTCACTATGTTAGGAT } \\
\text { R: TTTCCAATGGGTAAAGTATAACGTC }\end{array}$ & sequencing & 472 \\
\hline SLC25A46-8B & $\begin{array}{l}\text { F: CCCTGTGCAGAGTATGTTG } \\
\text { R: CCCAGTATTTCACTTGTATCCC }\end{array}$ & sequencing & 482 \\
\hline SLC25A46-8 & $\begin{array}{l}\text { F: CCCTGTGCAGAGTATGTTGG } \\
\text { R: AATGTGAAGGCGGTGCAAAA }\end{array}$ & qPCR & 118 \\
\hline SLC25A46-1_3 & $\begin{array}{l}\text { F: CGGACGGATTTGATGGCTTG } \\
\text { R: ATTGGCGGCGTAGAACAATG }\end{array}$ & splicing effect & 363 \\
\hline SLC25A46-1_2 & $\begin{array}{l}\text { F: GGAGGAACCCTTTTCCAGTG } \\
\text { R: AATGCAAGGATGTGCCAATA }\end{array}$ & qPCR & 133 \\
\hline TBP-5_6 & $\begin{array}{l}\text { F: CACGAACCACGGCACTGATT } \\
\text { R: TTTTCTTGCTGCCAGTCTGGAC }\end{array}$ & qPCR & 89 \\
\hline
\end{tabular}


Chapter

\section{Mutations in COX18 cause isolated complex IV deficiency, associated with neurodegeneration, chorea dystonic movement and type 1 diabetes}

Minh Nguyen, Irenaeus FM de Coo, Debby MEI Hellebrekers, Kees Schoonderwoerd, J. de Rijk- van Andel, Elvira Mulder, Rick Kamps, Bart de Koning, Mike Gerards ${ }^{*}$ and Hubert J M Smeets ${ }^{*}$

* These authors contributed equally to this work.

Manuscript submitted 


\section{ABSTRACT}

Cytochrome c oxidase (COX), or complex IV, is an essential regulator of oxidative phosphorylation catalyzing the terminal step of electron transfer in the mitochondria. Three complex IV subunits are encoded by the mtDNA and eleven by nuclear genes, assembled by more than 30 nuclear accessory factors. To date, pathogenic mutations have been identified in both mtDNA and nuclear genes, causing mitochondrial disorders with an isolated complex IV deficiency. We investigated a patient with slowly progressive neurodegeneration, cerebellar ataxia, chorea dystonic movements, polyneuropathy, type 1 diabetes and complex IV deficiency in muscle. Whole exome sequencing revealed compound heterozygosity for two splice site mutations (c.720G>A and c. $828+3 \mathrm{G}>\mathrm{C}$ ) in the COX18 gene, a complex IV assembly factor. The synonymous substitution c.720G>A (p.Glu240Glu) affected the last nucleotide of exon 4 and resulted in the deletion of this exon due to the loss of the donor splice site of intron 4, causing a frameshift and premature stop codon (p.Gly200Aspfs*79). The c.828+3G>C mutation on the other hand disrupted the splice donor site of intron 5 , resulting in an insertion of $4 \mathrm{bp}$ of intron 5 in the mRNA due to the activation of a cryptic donor site in intron 5, also leading to a frameshift and premature stop codon (p.Ser277Valfs*44). Total COX18 mRNA expression in patient fibroblasts was equal to the controls, but allele-specific mRNA analysis revealed that only $\sim 6 \%$ of wild type COX18 mRNA was present, indicating that the vast majority of COX18 mRNA was mutated and did not undergo nonsense-mediated mRNA decay. Also, protein levels were normal, implying that predominantly a mutant COX18 protein was produced, acting in a dominant negative manner, causing the phenotypes observed in our patient. This is the first report of COX18 mutations in a patient with isolated complex IV deficiency in muscle. 


\section{INTRODUCTION}

Cytochrome $c$ oxidase (COX) or complex IV is the last enzyme of the respiratory chain, and catalyzes the electron transfer from reduced cytochrome $\mathrm{c}$ to molecular oxygen. This reaction is coupled with the translocation of protons from the mitochondrial matrix into the intermembrane space, generating the proton-motive force that allows ATP to be synthesized by the ATP synthase (complex V). Mammalian COX is composed of 13 subunits, of both nuclear or mitochondrial origin. The three mitochondrial DNA encoded subunits (COX1, 2 and 3 ) form the catalytic core of the enzyme [1], whereas the 10 nuclear-encoded structural subunits (COX4, COX5A, COX5B, COX6A, COX6B, COX6C, COX7A, COX7B, COX7C and COX8) are required for structure and stability of complex IV $[2,3]$. The biogenesis of complex IV relies on a large number of accessory factors, which facilitate the assembly of the complex, but also other stages of the biogenesis, including transcription and mRNA maturation, translation of COX mitochondrial genes and processing, as well as import of nuclear encoded subunits into mitochondria and insertion of imported proteins into the mitochondrial inner membrane [3]. Mitochondrial disorders caused by isolated COX deficiency are fairly common. However, disease-causing mutations affecting structural subunits of COX have only been reported for mtDNA encoded genes MT-CO1, MT-CO2, MT-CO3 [4], MT-TS1 and MT-TL1 and nuclear encoded genes COX4I2 [5], COX6A1 [6], COX6B1 [7], COX7B [8], COX8A [9]. Complex IV deficiency is more often caused by mutations in genes encoding accessory proteins including NDUFA4 [10], COX10 [11], SCO1 [12], SCO2 [13], FASTKD2 [14], COX14 [15], COX20 [16], APOPT1 [17], COA3 [18], COX15 [19], COA5 [20], COA6 [21], SURF1 [22], TACO1 [23], and LRPPRC [24]. Cytochrome c oxidase deficiency is clinically heterogeneous, ranging from isolated myopathy to severe multisystem disease, with onset from infancy to adulthood [4], including Leigh syndrome due to SURF1 mutations [25], fatal infantile hypertrophic cardiomyopathy due to COX15 and SCO2 mutations [13, 19, 26], ketoacidotic coma and hepatopathy due to SCO1 mutations, tubulopathy and leukodystrophy due to COX10 mutations [27] and French-Canadian Leigh syndrome due to LRPPRC mutations [24]. Here, we report, for the first time, compound heterozygosity for 2 pathogenic mutations in the COX18 gene in a patient with isolated complex IV deficiency, who presented with slowly progressive neurodegeneration, cerebellar ataxia, chorea dystonic movements, polyneuropathy and type 1 diabetes.

\section{MATERIALS AND METHODS}

\section{Index patient}

The index patient was the only child of healthy non-consanguineous Dutch parents. She was born after a complicated pregnancy with a herpes zoster infection in the 7th 
month, but delivery was uncomplicated. Family history revealed that the father's mother was operated for aortic aneurysm. A brother of her maternal grandmother had two handicapped children with encephalopathy and calcification of the basal ganglia. No further diagnosis has been made. At the age of 2 years, she visited the outpatient clinic with a left handedness and complaints about delayed motor development. She was clumsy and showed an atactic, broad-based gait, but had no dysmorphic features or organ enlargements. In the following years, she gradually developed a spastic atactic movement disorder and a polyneuropathy. After she developed diabetes mellitus type I at the age of 7, a finger polyminimyoclonus was noted. Her mental abilities remained more or less constant with a verbal IQ of 99 and a performal IQ of 63. Physical examination revealed some halo naevi, but no other signs for non-neurological abnormalities. She was treated with neurostimulation that temporarily decreased the myoclonus. A Magnetic Resonance Imaging (MRI) of the brain performed at 7 years old showed diffuse cerebellar atrophy and abnormal hyperintensity at T2 sequence of the caudate nuclei and the putamen. A suspected mitochondrial origin of the disease was further enhanced by the results of the analysis of the muscle biopsy at 9 years, which showed some type grouping and lipid accumulation. No ragged red fibres or COX negative fibres were seen by electron microscopy. Deficiency of complex IV was detected in muscle: (at 9 years old) complex IV 42\%; Citrate synthase 89\% and (at17 years old): OXPHOS: complex IV 27\%; Citrate synthase 71\%. During puberty, her scoliosis th12-S1 was treated with a spondylodesis. In 2016, at the age of 26, she was seen at the outpatient clinic with a neurodegenerative disease and a total IQ of 55 (verbal 61 and performal 54). She suffered from severe vision loss, a severe cerebellar ataxia, dysarthic speech, polyneuropathy and diabetes mellitus. She also had obstructive apneu syndrome with hypoventilation.

\section{Whole-exome sequencing and data analysis}

Whole-exome sequencing was performed as previously described [28]. The effect of splice-site mutations was predicted by algorithms integrated in the Alamut software version 2.4 (SpliceSiteFinder-like, MaxEntScan, NNSplice, GeneSplicer, and Human Splicing Finder).

\section{COX18 mRNA analyses}

Human fibroblasts were cultured in Dulbecco modified Eagle (DMEM) supplemented with 10\% fetal bovine serum (FBS), 1\% I-glutamine, and 1\% penicillin/streptomycin (Gibco BRL, Carlsbad, CA, USA) and incubated in humidified atmosphere of 5\% CO2 at $37{ }^{\circ} \mathrm{C}$. Total RNA was isolated in triplicate from cultured fibroblasts using phenolchloroform extraction and ethanol precipitation. Subsequently, cDNA was synthesized using the RT First Strand Kit with oligo (dT) primers (Qiagen, The Netherlands). To evaluate the effect of the COX18 mutations on splicing, reverse transcription polymerase chain reaction (RT-PCR) products spanning exon 3-exon 6 were analyzed. The amplified 
fragments were separated on $2 \%$ agarose gel and sequenced to identify the variations in the mRNA transcript. Sequencing reactions were carried out according to the Sanger dideoxy chain termination method using the BigDye ${ }^{\circledR}$ terminator v1.1 cycle sequencing kit (Applied Biosystems). Sequence analyses were carried out with 3730XL DNA Analyser (Applied Biosystems). Real-time quantitative polymerase chain reaction (qPCR) with specific primers were designed to quantify wild-type, mutant or total COX18 transcripts. TBP was used as the reference gene for normalization. The sequences and positions of the primers and the product sizes of the RT-PCR are shown in Table 1

Table 1: Sequences of primers used for RT-PCR validation of alternative splicing events and for qPCR of COX18

\begin{tabular}{|c|c|c|c|}
\hline \multirow{2}{*}{$\begin{array}{l}\text { Gene (exon) } \\
\text { Cox18 } \\
\text { (exon 3- exon 6) }\end{array}$} & \multicolumn{2}{|c|}{ Primer sequence } & \multirow{2}{*}{$\begin{array}{l}\text { Length }(\mathrm{bp}) \\
401\end{array}$} \\
\hline & $\begin{array}{l}\mathrm{F} \\
\mathrm{R}\end{array}$ & $\begin{array}{l}\text { 5' - GCCACTGTGTTGGTTTGGAT -3' } \\
\text { 5' - CGAAATCCAGGAGAACGCAG -3' }\end{array}$ & \\
\hline primers for qPCR & & & \\
\hline cox18 (exon 3) & $\begin{array}{l}\mathrm{F} \\
\mathrm{R}\end{array}$ & $\begin{array}{l}\text { 5' - GCCACTGTGTTGGTTTGGAT -3' } \\
\text { 5' - CCCGTGCTTAAATTCCGGAG -3' }\end{array}$ & 74 \\
\hline cox18 (exon 4) & $\begin{array}{l}\mathrm{F} \\
\mathrm{R}\end{array}$ & $\begin{array}{l}\text { 5'- GTTTCCTGACCTCACTGCAC -3' } \\
\text { 5'- CGCCAACAGAGATAGGCAGA -3' }\end{array}$ & 56 \\
\hline $\begin{array}{l}\text { cox18 } \\
\text { (exon 4-exon5/exon6 } \\
\text { boundary) }\end{array}$ & $\begin{array}{l}\mathrm{F} \\
\mathrm{R}\end{array}$ & 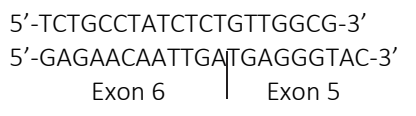 & 163 \\
\hline $\begin{array}{l}\text { TBP } \\
\text { (exon 5- exon 6) }\end{array}$ & $\begin{array}{l}F \\
R\end{array}$ & $\begin{array}{l}\text { 5' - CACGAACCACGGCACTGATT -3' } \\
5^{\prime} \text { - TTTTCTTGCTGCCAGTCTGGAC - } 3^{\prime}\end{array}$ & 89 \\
\hline
\end{tabular}

\section{Western blot analysis}

Fibroblasts were grown in T75 flasks, trypsinized, and pelleted for protein isolation. Total protein from cell pellets was isolated as described (PMID:10556302). The total amount of protein in each sample was determined using the Bradford assay (BioRad, Hercules, CA, USA). A $30 \mu \mathrm{g}$ protein extracts per lane were separated by SDSpolyacrylamide gel electrophoresis on a 4-15\% Tris-Glycine extended gel (BioRad) and transferred to a PVDF membrane. The membrane was blocked with $5 \%$ nonfat milk (Sigma-Aldrich) and incubated overnight at $4^{\circ} \mathrm{C}$ with the primary antibodies rabbit-antiCOX18 (clone ab184773, Abcam) and mouse-anti-SDHA (clone ab14715, Abcam). After 3 washes in PBS with $0.1 \%$ Tween-20, the membrane was incubated with the antimouse or anti-rabbit secondary antibodies conjugated to horseradish peroxidase (Dako) and proteins were visualized using chemiluminescence (ECL, Thermo Scientific). 


\section{RESULTS}

Exome sequencing was performed in a Dutch non-consanguineous patient with slowly progressive neurodegeneration, cerebellar ataxia, chorea dystonic movements, polyneuropathy and type 1 diabetes and isolated complex IV deficiency in muscle (Fig. 1A). A total of 24,144 variants were detected. After excluding non-splice site-related intronic variants, UTR-variants, synonymous variants, variants with an allele frequency $>0.01$ in dbSNP137 and our in-house database, a total of 9 possible candidate genes remained, carrying at least two possibly pathogenic mutations: KIF15 (c.632C>T; NM_020242.2), CNTRL (c.4319G>C, c.1622A>T, c.2861T>C; NM_007018.4), COX18 (c.720G>A, c.828+3G>C; NM_173827.3), FAT3 (c.2681G>A, c.5666A>G; NM_001008781.2), FLG (c.9565G>C, c.309G>A; NM_002016.1), HSPG2 (c.3056C $>$ T, c.3707C $>A$, c.3781_3782insA>CA; NM_005529.6), LRIF1 (c.1592C>A, c.482C>G; NM_018372.3), OR2Z1 (c.393_394insG>GT, c.396_397insT>TGAGGG; NM_001004699.2), WNK3 (c.2413G>C, c.5077T>G; NM_020922.4). Only COX18 was related to complex IV and was therefore the prime candidate to proceed with. The mutations in COX18 were a splice site mutation in intron 5 (c.828+3G>C; NM_173827.3) and a synonymous mutation at the end of exon 4, which belonged to the conserved splice donor sequence (c.720G>A; p.Glu240Glu; NM_173827.3) (Fig.1B). Splice site prediction programs for the c. $828+3$ G>C mutation (MaxEnt: $-100 \%$, NNSPLICE: $-96.6 \%$ ) and for the c.720G>A mutation (MaxEnt: $-86.8 \%$, NNSPLICE: -76.9\%) indicated that the normal splice site was abolished and an alternative splice site could be used, in both cases predicted to cause a frameshift and premature stop codon (Fig. 1C). Sanger sequencing showed that the father was heterozygous for the $c .828+3 \mathrm{G}>\mathrm{C}$ mutation and the mother for the c.720G >A mutation, indicating that the patient was compound heterozygous.

RT-PCR was performed to confirm the predictions of the splice site programs. A segment of COX18 cDNA encompassing exons 3-6 was amplified from total RNA extracted from fibroblasts of a control and the patient to test the effect of the c.720G $>A$ and c. $828+3 \mathrm{G}>\mathrm{C}$ mutations. In the control, only one PCR product of expected size corresponding to the correctly spliced cDNA was observed, whereas two bands were detected in the patient (Fig. 2A). Sequencing of the lower band from the patient revealed a deletion of exon 4 as a result of the c.720G>A mutation. This deletion removed a 125bp fragment of mRNA, leading to a frameshift and premature stop codon (p.Ala200Aspfs*79) (Fig. 2B_1). No wild-type COX18 mRNA was detected by sequence analysis. Sequence of the upper band showed an insertion of $4 \mathrm{bp}$ of intron 5 as a result of the $c .828+3 \mathrm{G}>\mathrm{C}$ mutation resulting in a frameshift and premature stop codon ( $\mathrm{p}$. Ser277Valfs*44) (Fig. 2B_2). Wild-type COX18 mRNA sequence was detectable by sequence analysis as well.

The COX18 mRNA level was normal in the patient fibroblasts compared with control, suggesting mutant transcripts were not degraded by nonsense-mediated mRNA decay (Fig. 3A). We used primers in exon 4 to determine the level of mRNA of the c.720 G>A 
allele causing the deletion of exon 4 and the relative quantity was $28 \%$ compared with control. Quantification of the wild-type COX18 transcript using a forward primer located in exon 4 and a reverse primer spanning the exon 5- exon 6 boundary, which is specific for the wild-type transcript, showed that only $6 \%$ of wild-type COX18 mRNA was present in the total mRNA. Therefore, the proportion of the $c .828+3 \mathrm{G}>\mathrm{C}$ transcript was $66 \%$ of the total RNA (Fig. 3A). To investigate the COX18 protein expression, we examined the levels of COX18 protein extracts in fibroblasts of the patient and control using immunoblotting assays. A similar amount and size of COX18 protein was observed in the patient and the control fibroblasts (Fig. 3B).

\section{(A)}
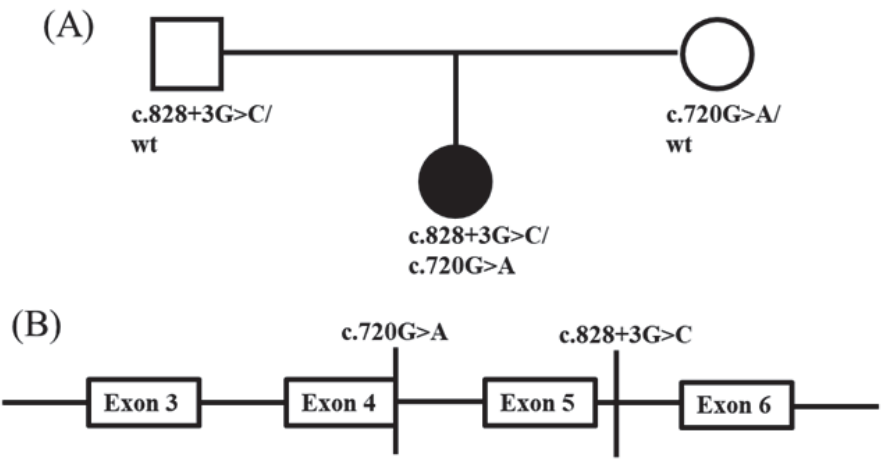

(C) NM_173827.3:c.720G>A

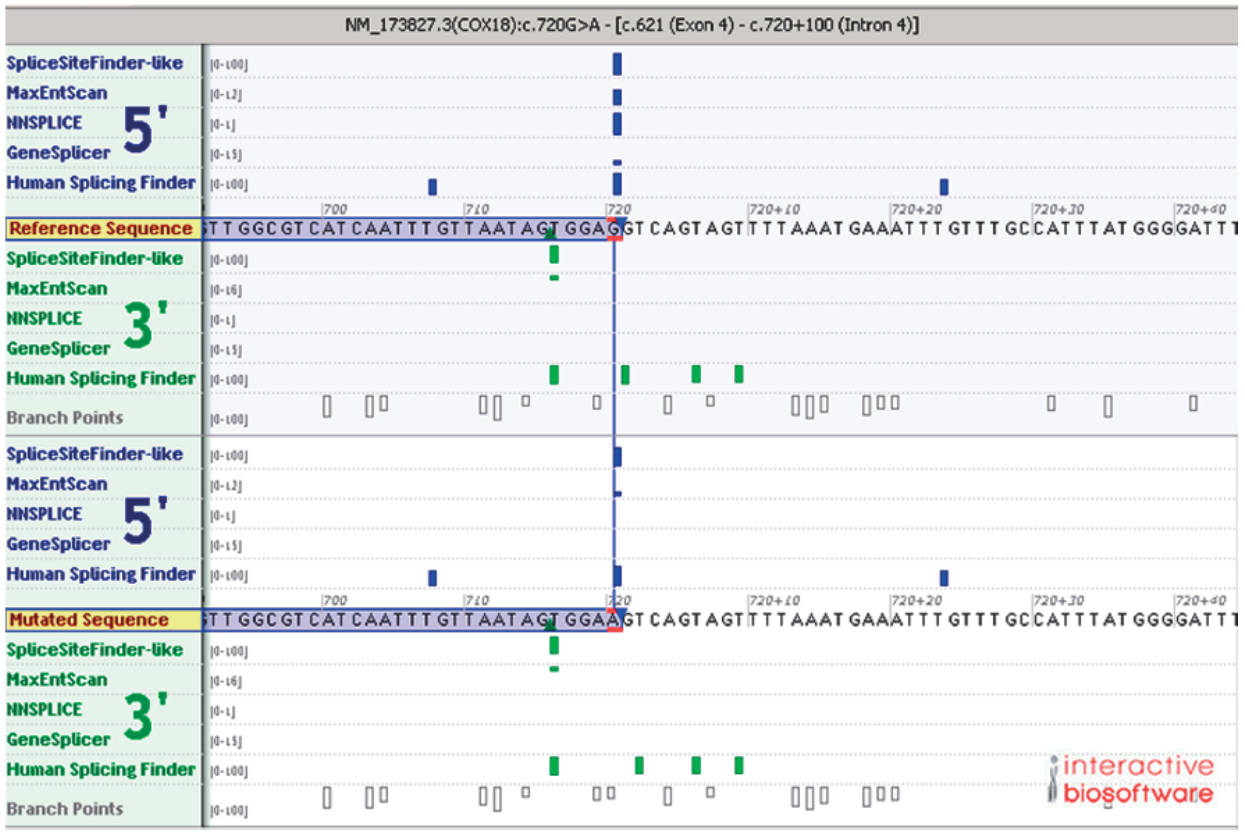


NM_173827.3:C.828+3G>C

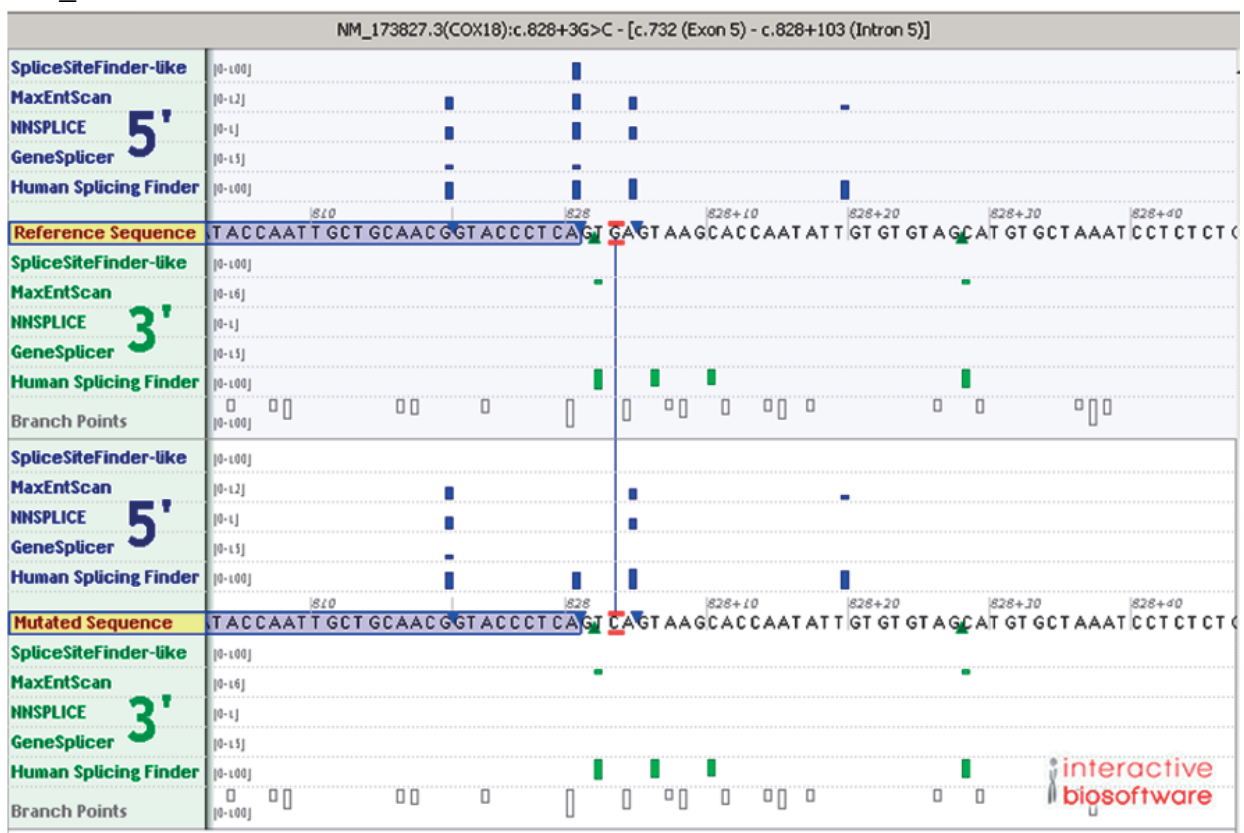

Fig. 1: Pedigree of family and in silico predictions of the COX18 mutations

A) Pedigree of a Dutch non-consanguineous family. Index patient with neurodegeneration, chorea dystonic movement and type 1 diabetes depicted by the black symbol. (B) Schematic diagram showing the positions of two COX18 mutations and exon 6 (the last exon). (C) a graphical display from the Alamut biosoftware interface shows splice site scores for the wild-type and the c.720G>A and c.828+3G>C mutants in the COX18 gene, calculated with four algorithms: SpliceSiteFinder-like, MaxEntScan, NNSPLICE, and GeneSplicer. An abolition of normal splicing and the use of alternative splice sites is predicted by these tools. Exons are drawn as blue boxes. Predicted splice sites from SpliceSiteFinder-like, MaxEntScan, NNSPLICE, GeneSplicer and Human Splicing Finder are displayed as blue vertical bars for 5' (donor) sites, and as green vertical bars for 3' (acceptor) sites. The height of each bar is proportional to the maximum possible score computed by the corresponding algorithm. 
(A)

(B1)

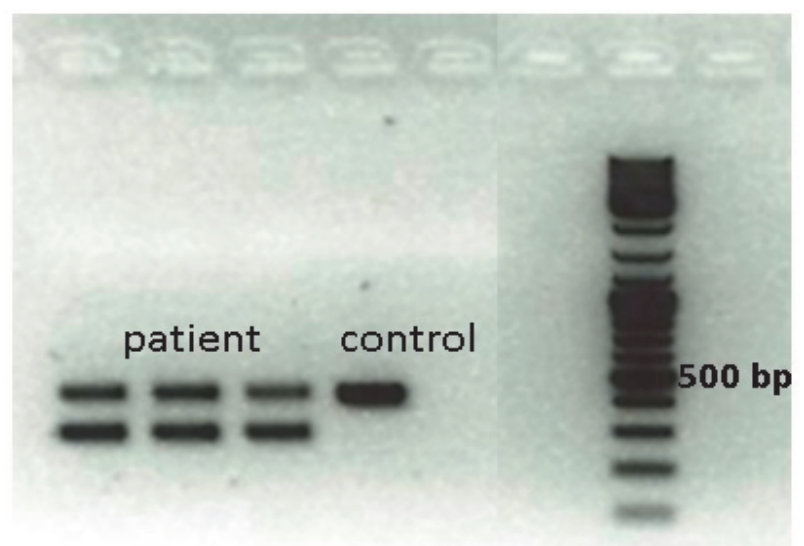

Donor splice site

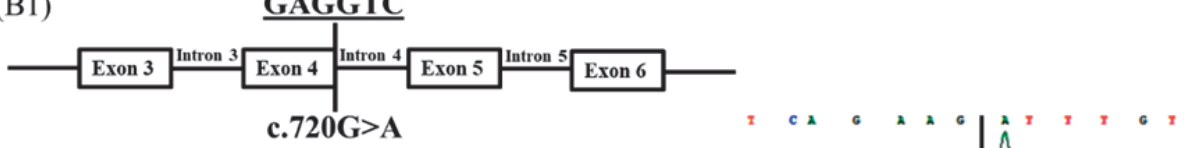

Alternative splice site

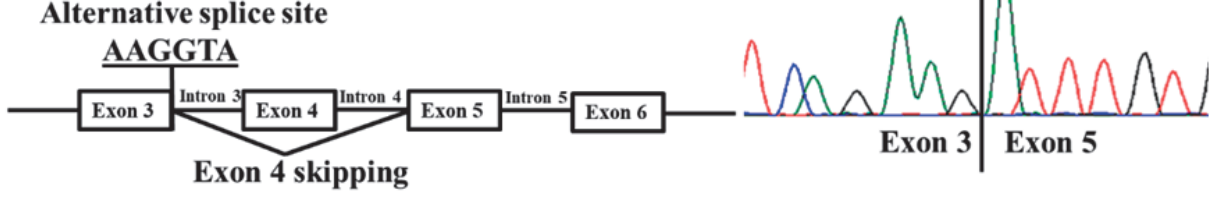

(B2)

Donor splice site

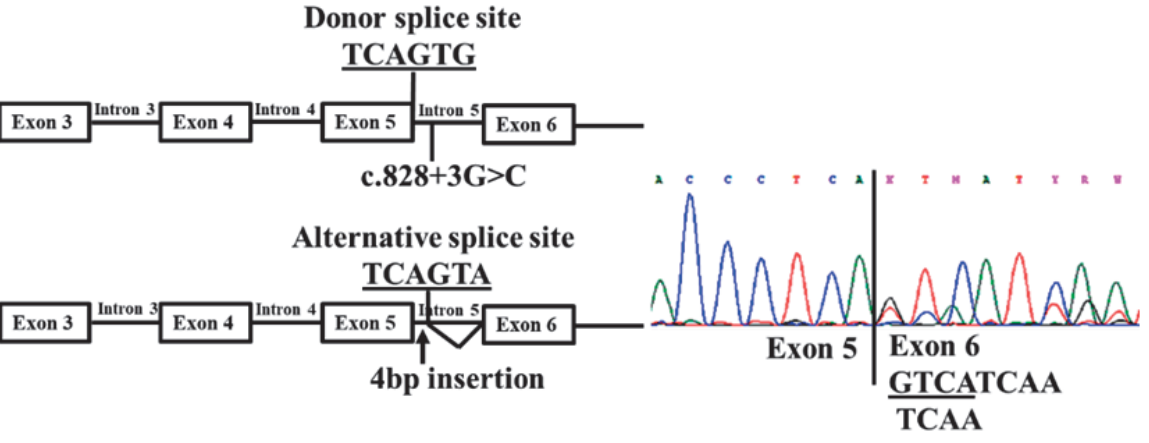

Fig 2: The effects of COX18 mutations on RNA splicing. (A) PCR products of CDNA spanning exon3-exon 6. The control shows the expected wild-type size, whereas the patient has an additional transcript product. (B) Schematic representation of the transcripts resulting from incorrect splicing. Sequence analysis of the lower band demonstrates a deletion of exon 4 (B1) and the upper band shows a heterozygous/mixed sequence due to 4 bp insertion of intron 5 (B2). 
(A)

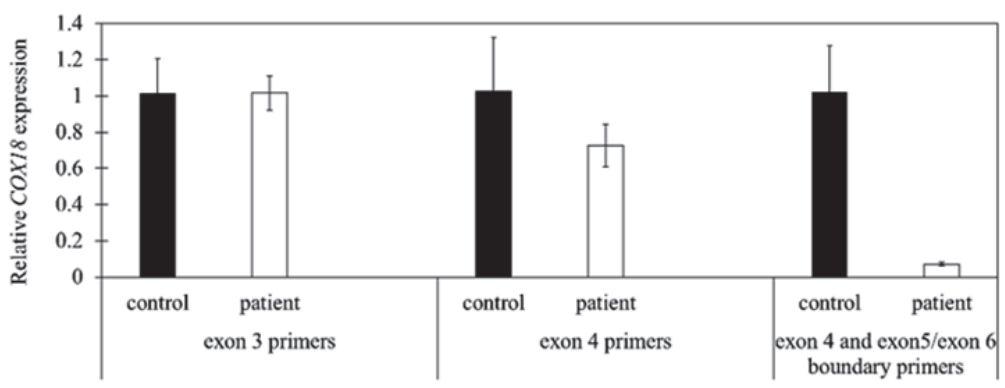

(B)

$\begin{array}{llllll}1 & 2 & 3 & 4 & 5 & 6\end{array}$

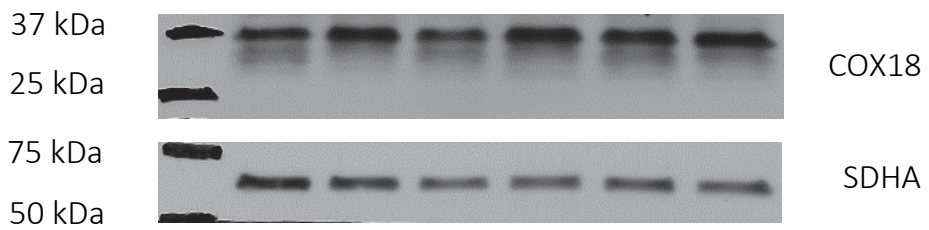

Fig. 3 Functional study of COX18 mutations at the RNA and protein levels in patient and control.

(A) COX18 mRNA expression in fibroblasts of the patient and control is assessed by quantitative RT-PCR using primer pairs specific for total COX18 mRNA (exon 3), COX18 mRNA with deletion exon 4 (exon 4), and wildtype COX18 mRNA (exon 4 and exon5/exon 6 boundary) with TBP transcript as endogenous control. Total COX18 mRNA expression of the patient fibroblasts is equal to the control. There is almost no wild-type transcript ( 6\%) in patient fibroblasts, which means 94\% of COX18 transcript is mutant. Experiments were performed in triplicate. Error bars indicate standard deviation.

(B) Western blotting results show similar protein levels in three independent protein isolates for control (lane $1,3,5)$ and patient (lane 2, 4, 6) fibroblasts. Molecular-mass standards (KDa) are indicated.

\section{DISCUSSION}

Whole exome sequencing was performed in a patient from non-consanguineous parents presenting with slowly progressive neurodegeneration, cerebellar ataxia, chorea dystonic movements, polyneuropathy, type 1 diabetes and complex IV deficiency. Two heterozygous splice site mutations were identified in the COX18 gene (c.720 G>A and c. $828+3 \mathrm{G}>\mathrm{C}$ ). The c.720 G>A mutation was an exonic synonymous substitution at the last nucleotide of the exon 4, which was highly conserved throughout evolution and disrupted the donor splice site. Mutation c. $828+3 \mathrm{G}>\mathrm{C}$ resulted in the insertion of $4 \mathrm{bp}$ of intron 5. Both mutations lead to alternative splicing and premature stop codons. Transcripts that harbor a premature stop codon can be degraded by an mRNA surveillance pathway termed nonsense-mediated mRNA decay (NMD). However, NMD is not triggered when a premature stop codon is located downstream of the last exon junction complex (EJC) [29]. In the case of COX18, both mutations introduced premature stop codons into the final exon and total mRNA of the patient fibroblasts was expressed at a 
level similar to the control indicating that NMD did not occur. Interestingly, wild-type specific qPCR revealed that almost no wild-type transcript ( $\sim \%)$ was present in patient fibroblasts, which means $\sim 94 \%$ of COX18 transcript is mutant, $28 \%$ with skipped exon 4 and $66 \%$ with the $4 \mathrm{bp}$ insertion of intron 5 .

Human COX18 consists of 333 amino acids containing 5 transmembrane helices. The c.720 G>A mutation leads to exon 4 skipping, which is a central part of COX18 protein, and a shift in reading frame resulting in an altered nonsense protein with premature stop codon (p.Gly200Aspfs*79), 277 amino acids in size. For transcript c.828+3 G>C exon 4 is contained, however, this insertion of 4 base pairs of intron 5 also results in an altered protein with a premature stop codon (p.Ser277Valfs*44), 319 amino acids in size. The truncated proteins with an altered amino acid sequence, starting from the site of the mutation, lack the fifth transmembrane domain and $\mathrm{C}$ terminal tail. Based on the gene expression data, the amounts of aberrant truncated proteins could be comparable to the amount of wt COX18 in controls. Indeed, equal COX18 protein expression was detected in the patient and control fibroblasts using Western Blot analysis. Since only $6 \%$ of wild-type transcript was present in patient fibroblasts, it is unlikely that protein detected in Western Blot is wild-type. If $4 \mathrm{bp}$ - insertion transcript was translated into a stable protein, it would be only 13 amino acids shorter than normal. On an SDS-PAGE gel, such a protein would be indistinguishable from the wild-type protein (37kDa versus $35.4 \mathrm{kDa}$ ). The exon 4 deletion on the other hand would result in a protein with a predicted size of 277 amino acid $(30.8 \mathrm{kDa})$, which is smaller compared to normal COX18 (333 amino acids, 37kDa). Interestingly, the protein resulting from the exon 4 deletion was not observed on Western blot, despite the COX18 antibody targeted amino acids encoded by exon 1 . This indicates the exon 4 deleted mRNA is not translated or the resulting protein is unstable and rapidly turned over.

COX20 is the first chaperone that interacts with and stabilizes newly synthesized COX2, by facilitating COX2 N-terminus insertion into the mitochondrial inner membrane, and remains associated with COX2 in its maturation. Subsequently, COX18 catalyzes the proper translocation of the second, C-terminus transmembrane of COX2 across the mitochondrial inner membrane. The release of COX18 from the complex probably coincides with the incorporation of SCO1. This protein together with SCO2 and COA6 interact with COX2 to enable copper transfer and complete COX2 maturation [30]. As the mutated COX18 protein lack the entire $5^{\text {th }}$ transmembrane protein and Cterminal end, it is likely that the translocation of COX2 across the mitochondrial inner membrane will be disturbed. Based on these data, the mutations in the COX18 gene likely explain the severe complex IV deficiency observed in muscle in our patient. As the fibroblasts of the patient exhibit normal enzyme activity, we could not perform a complementation study. The reason for the difference between muscle and fibroblasts is not clear, but similar discrepancies have been reported before in patients with complex IV deficiency [31, 32]. Mitochondrial defects tend to affect tissues with a high energy demand such as the nervous system, heart and muscle. The tissue-specific pattern 
probably reflects different demands for the regulation of COX biogenesis. The resulting protein may still be partially functional or the remaining wild-type protein may provide sufficient activity to support cell types with low energy requirements, such as fibroblasts. Further experimental investigation in fibroblasts and muscle are needed to evaluate their true biological effects. Based on the results obtained in this study, it is highly likely that that the COX18 splice mutations identified in our patient caused the complex IV deficiency and phenotypes in our patient. To our knowledge, this is the first case of a patient with COX18 mutations, adding COX18 as a new complex IV disease gene. 


\section{REFERENCES}

1. Capaldi, R.A., Structure and function of cytochrome c oxidase. Annu Rev Biochem, 1990. 59: p. 569-96.

2. Fornuskova, D., et al., Novel insights into the assembly and function of human nuclear-encoded cytochrome c oxidase subunits 4, 5a, 6a, 7a and 7b. Biochem J, 2010. 428(3): p. 363-74.

3. Stiburek, L., et al., Biogenesis of eukaryotic cytochrome c oxidase. Physiol Res, 2006. 55 Suppl 2: p. S2741.

4. Shoubridge, E.A., Cytochrome c oxidase deficiency. Am J Med Genet, 2001. 106(1): p. 46-52.

5. Shteyer, E., et al., Exocrine pancreatic insufficiency, dyserythropoeitic anemia, and calvarial hyperostosis are caused by a mutation in the COX412 gene. Am J Hum Genet, 2009. 84(3): p. 412-7.

6. Tamiya, G., et al., A mutation of COX6A1 causes a recessive axonal or mixed form of Charcot-MarieTooth disease. Am J Hum Genet, 2014. 95(3): p. 294-300.

7. Massa, V., et al., Severe infantile encephalomyopathy caused by a mutation in COX6B1, a nucleusencoded subunit of cytochrome c oxidase. Am J Hum Genet, 2008. 82(6): p. 1281-9.

8. Indrieri, A., et al., Mutations in $C O X 7 B$ cause microphthalmia with linear skin lesions, an unconventional mitochondrial disease. Am J Hum Genet, 2012. 91(5): p. 942-9.

9. Hallmann, K., et al., Loss of the smallest subunit of cytochrome c oxidase, COX8A, causes Leigh-like syndrome and epilepsy. Brain, 2016. 139(Pt 2): p. 338-45.

10. Pitceathly, R.D., et al., NDUFA4 mutations underlie dysfunction of a cytochrome c oxidase subunit linked to human neurological disease. Cell Rep, 2013. 3(6): p. 1795-805.

11. Antonicka, H., et al., Mutations in COX10 result in a defect in mitochondrial heme A biosynthesis and account for multiple, early-onset clinical phenotypes associated with isolated COX deficiency. Hum Mol Genet, 2003. 12(20): p. 2693-702.

12. Valnot, I., et al., Mutations of the SCO1 gene in mitochondrial cytochrome c oxidase deficiency with neonatal-onset hepatic failure and encephalopathy. Am J Hum Genet, 2000. 67(5): p. 1104-9.

13. Papadopoulou, L.C., et al., Fatal infantile cardioencephalomyopathy with COX deficiency and mutations in SCO2, a COX assembly gene. Nat Genet, 1999. 23(3): p. 333-7.

14. Ghezzi, D., et al., FASTKD2 nonsense mutation in an infantile mitochondrial encephalomyopathy associated with cytochrome c oxidase deficiency. Am J Hum Genet, 2008. 83(3): p. 415-23.

15. Weraarpachai, W., et al., Mutations in C12orf62, a factor that couples COX I synthesis with cytochrome c oxidase assembly, cause fatal neonatal lactic acidosis. Am J Hum Genet, 2012. 90(1): p. 142-51.

16. Szklarczyk, R., et al., A mutation in the FAM36A gene, the human ortholog of COX20, impairs cytochrome c oxidase assembly and is associated with ataxia and muscle hypotonia. Hum Mol Genet, 2013. 22(4): p. 656-67.

17. Melchionda, L., et al., Mutations in APOPT1, encoding a mitochondrial protein, cause cavitating leukoencephalopathy with cytochrome c oxidase deficiency. Am J Hum Genet, 2014. 95(3): p. 315-25.

18. Ostergaard, E., et al., Mutations in COA3 cause isolated complex IV deficiency associated with neuropathy, exercise intolerance, obesity, and short stature. J Med Genet, 2015. 52(3): p. 203-7.

19. Antonicka, H., et al., Mutations in COX15 produce a defect in the mitochondrial heme biosynthetic pathway, causing early-onset fatal hypertrophic cardiomyopathy. Am J Hum Genet, 2003. 72(1): p. 10114.

20. Huigsloot, M., et al., A mutation in C2orf64 causes impaired cytochrome c oxidase assembly and mitochondrial cardiomyopathy. Am J Hum Genet, 2011. 88(4): p. 488-93.

21. Baertling, F., et al., Mutations in COA6 cause cytochrome c oxidase deficiency and neonatal hypertrophic cardiomyopathy. Hum Mutat, 2015. 36(1): p. 34-8.

22. Zhu, Z., et al., SURF1, encoding a factor involved in the biogenesis of cytochrome c oxidase, is mutated in Leigh syndrome. Nat Genet, 1998. 20(4): p. 337-43.

23. Weraarpachai, W., et al., Mutation in TACO1, encoding a translational activator of COXI, results in cytochrome c oxidase deficiency and late-onset Leigh syndrome. Nat Genet, 2009. 41(7): p. 833-7. 
24. Olahova, M., et al., LRPPRC mutations cause early-onset multisystem mitochondrial disease outside of the French-Canadian population. Brain, 2015. 138(Pt 12): p. 3503-19.

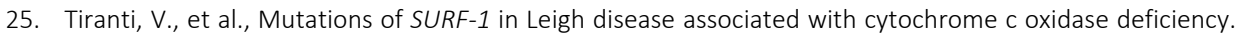
Am J Hum Genet, 1998. 63(6): p. 1609-21.

26. Jaksch, M., et al., Mutations in $\mathrm{SCO} 2$ are associated with a distinct form of hypertrophic cardiomyopathy and cytochrome c oxidase deficiency. Hum Mol Genet, 2000. 9(5): p. 795-801.

27. Valnot, I., et al., A mutation in the human heme A:farnesyltransferase gene (COX10) causes cytochrome c oxidase deficiency. Hum Mol Genet, 2000. 9(8): p. 1245-9.

28. Nguyen, M., et al., Pathogenic CWF19L1 variants as a novel cause of autosomal recessive cerebellar ataxia and atrophy. Eur J Hum Genet, 2016. 24(4): p. 619-22.

29. Lindeboom, R.G., F. Supek, and B. Lehner, The rules and impact of nonsense-mediated mRNA decay in human cancers. Nat Genet, 2016. 48(10): p. 1112-8.

30. Bourens, M. and A. Barrientos, Human mitochondrial cytochrome c oxidase assembly factor COX18 acts transiently as a membrane insertase within the subunit 2 maturation module. J Biol Chem, 2017. 292(19): p. 7774-7783.

31. Stiburek, L., et al., Tissue-specific cytochrome c oxidase assembly defects due to mutations in SCO2 and SURF1. Biochem J, 2005. 392(Pt 3): p. 625-32.

32. Stroud, D.A., et al., COA6 is a mitochondrial complex IV assembly factor critical for biogenesis of mtDNAencoded COX2. Hum Mol Genet, 2015. 24(19): p. 5404-15. 


\section{Chapter}

\section{Whole exome sequencing reveals HKDC1 and MED20 as candidate genes in a child with a neurodegenerative disorder and congenital \\ cataract}

Minh Nguyen, Jo Vanoevelen, Joost Nicolai, Ivo Eijkenboom, Rick Kamps, Constance T.R.M. Stumpel, Rinske F. Bos, Helene Pendeville, Hubert JM Smeets, Debby MEI Hellebrekers* and Mike Gerards*

* These authors contributed equally to this work.

Manuscript in preparation 


\section{ABSTRACT}

Neurodegenerative diseases are characterized by a progressive loss of structure or function of neurons. A large number of neurodegenerative diseases have been described, many of which have a prenatal, neonatal or early-infantile onset. Due to the loss of neurons, the brain volume is decreased often resulting in microcephaly. In a child of consanguineous parents of Turkish descent, presenting with cataract, severe psychomotor regression with spasticity, swallowing problems and epilepsy and secondary microcephaly, we performed homozygosity mapping combined with whole exome sequencing. The patient was homozygous for two variants in two different candidate genes. In the HKDC1 gene, a novel homozygous nonsense mutation was identified (c.1441 C>T, p.R481X). No mutations have been reported before in this gene. Additionally, a homozygous missense mutation, with a predicted damaging effect on the protein, was identified in MED20 (c.233 T>C, p. L78P). Homozygosity for a MED20 variant (p.Gly114Ala) has been reported once in 2 affected siblings with infantile basal ganglia degeneration leading to dystonia and brain atrophy. The oldest of these two girls showed secondary microcephaly and cataract. A definite conclusion on the involvement of MED2O could not be drawn, as the findings were gathered from one family and based on prediction models only. As the clinical symptoms of our patients were different, we investigated also the effect of HKDC1 mutation in further detail. Knockdown of the $H K D C 1$ gene in zebrafish with morpholinos led to neurodevelopmental defects including small heads and small eyes. Based on this data, both genes might be involved in the clinical symptoms of the patient, but a definite conclusion cannot be drawn and requires the identification of mutations in either or both genes in other patients with the same phenotype. 


\section{INTRODUCTION}

Neurodegenerative diseases are characterized by a progressive loss of structure or function of neurons. A large number of neurodegenerative diseases have been described, many of which have a prenatal, neonatal or early-infantile onset. Due to loss of neurons, the brain volume is decreased, often resulting in microcephaly. In some metabolic disorders, however, loss of neurons leads to an increased brain volume (megencephaly), resulting in macrocephaly. For practical reasons, primary and secondary microcephaly are distinguished. Primary microcephaly is defined by a skull circumference below -3 SD beneath the mean from birth and is often related to genetic disorders, but can also be due to infections early in pregnancy. Secondary microcephaly implicates that the skull circumference was still normal at birth and microcephaly has presented later in life. Secondary microcephaly can be caused by acquired disorders like meningitis, stroke or post-asphyxia encephalopathy or genetic or metabolic disorders [1].

Additional clinical information can help to establish an etiological diagnosis in neurodegenerative disease. Especially, ophthalmological evaluation can be helpful as the presence of cataract, corneal clouding, glaucoma, cherry red spot, retinal or macular degeneration can reveal a diagnosis or pathophysiological mechanism. Many children with neurodegenerative disorders still remain genetically undiagnosed. Novel genes and gene defects linked to neurodegenerative disorders are continuously being identified, mostly due to whole exome sequencing (WES). WES has become an integral part of medical diagnosis, as it is getting cost-effective and commercial available. WES is genotype driven and allows the detection of disease causing mutations in an unbiased manner independent of the phenotype. This is particularly important to establish genetic diagnoses in individuals with rare or complex phenotypes and in case of genetic heterogeneous disorders or phenotypes.

Here, we describe a deceased child, who presented with bilateral cataract, severe psychomotor regression with spasticity, swallowing problems and epilepsy and secondary microcephaly, in which WES revealed possible pathogenic variants in the HKDC1 and MED20 genes.

\section{MATERIALS AND METHODS}

\section{Patient}

The patient was a male child of parents of Turkish descent, who were first cousins. He presented at birth with respiratory problems due to meconium aspiration congenital cataract. At the age of 5-months hypotonia and a developmental delay was noted. Additionally, he developed secondary microcephaly, spasticity, therapy resistant epilepsy 
and feeding and swallowing problems. MRI at the age of 5-months revealed nonspecific abnormalities with severe cerebral and cerebellar atrophy involving loss of both white and gray matter and mild ventriculomegaly due to neuronal loss. There was an evident delay in myelination. He was admitted many times because of respiratory problems due to aspiration. The patient died at the age of 4 years. The postmortem MRI showed severe cerebral and cerebellar atrophy of both extensive white and gray matter volume loss.

While seven siblings of the patient were healthy, six siblings from the mother had deceased at the age of 10 days-18 months from an anamnestically similar phenotype, although no objective records exist. Family history showed that the mother originated from a consanguineous marriage as. Unfortunately, no material and clinical data were preserved from the mother's siblings in Syria.

\section{Homozygosity mapping}

Homozygosity mapping was performed in the index patient and parents, using the Affymetrix GeneChip Human Mapping 250 K Nspl SNP array according to manufacturers' instructions. The data was subsequently analyzed with HomozygosityMapper to identify homozygous regions (http://www.homozygositymapper.org/) [2].

\section{Whole exome sequencing}

Whole exome sequencing was performed as previously described [3]. As the index is the offspring of a consanguineous marriage, exome sequencing variants were first filtered for homozygous variants, either novel or present in the dbSNP and/or ExAc database with a frequency $<0.01$, predicted damaging function (nonsense, missense, and splice variants) and for presence in the homozygous regions which were identified from both homozygosity mapping and stretches of homozygous variants found in the WES data. Candidate disease-causing variants were confirmed using Sanger sequencing. Sequencing reactions were carried out according to the Sanger dideoxy chain termination method using the BigDye ${ }^{\circledR}$ terminator v1.1 cycle sequencing kit (Applied Biosystems). Sequence analyses were carried out with 3730XL DNA Analyser (Applied Biosystems). Segregation was also verified for all family members. The sequences and positions of the primers and the product sizes of the PCR are shown in Table 1. 
Table 1: Sequences of primers with M13 tails used for PCR validation and segregation of variants in MED2O and HKDC1.

\begin{tabular}{llll}
\hline Gene & \multicolumn{2}{l}{ Primer sequence 5'-3' } & $\begin{array}{l}\text { Length } \\
\text { (bp) }\end{array}$ \\
\hline MED20 & F & TGTAAAACGACGGCCAGTCGGGAAGCTGATGTATGTGA & 151 \\
& R & CAGGAAACAGCTATGACCCTTGCTGGCCTTAGCACTCT & \\
HKDC1 & F & TGTAAAACGACGGCCAGTGAGCCTGGCCTTTATGATTG & 474 \\
& R & CAGGAAACAGCTATGACCCTTGCCCTCCATTAGAGACG & \\
\hline
\end{tabular}

HKDC1 knockdown in zebrafish

Two different MOs were designed to target the exon9-intron9 (E9I9) and exon10intron10 (E10I10) splice junction of the HKDC1 gene (Gene Tools, Inc., Philomath, OR). The sequence of the HKDC1 E9I9 morpholino is CATTTGAGGAGAACTGACGTACTGT, and the sequence of the HKDC1 E10I10 morpholino is GGTATTTTCTATTACCTGTCCCATC. Morpholinos at doses ranging from 0.5-6 ng MO/embryo were injected into 1- to 2-cell stage embryos. At 2 days post fertilization, RNA was extracted from embryos injected with the HKDC1 E9I9 MO and the HKDC1 E10I10 MO using TRIzol reagent (Life Technologies, Grand Island, NY) according to the manufacturer's instructions, followed by a cleaned up using the Qiagen RNEasy Mini kit. cDNA was synthesized using qScript cDNA synthesis kit. The efficacy of MOs was tested by PCR using primers (HKDC1 F: AGAATGGCCAAGAAGGGTCT; HKDC1_R: GCCAGTCCGAATCTTAACCA) which span the intron/exon boundary targeted by the MOs. Quantification of expression levels of HKDC1 (HKDC1_F: TTGATAAGGGAGATCTGGTCTG; HKDC1_R: TCAGCATGTCCACAACATCA) was performed by real-time quantitative PCR on the 7900HT Fast Real-Time PCR System (Life Technologies) using a SYBR Green qPCR Kit. All measurements were done in triplicate. ODC1 (ODC1_F: GTGGGCGACTGGCTGCTGTT; ODC1_R: CCGCAGTGGGATGGCACGTT) was used to normalize expression levels.

\section{RESULTS}

Homozygosity mapping and whole exome sequencing analysis

Homozygosity mapping was performed using DNA samples of the patient and parents, which revealed two unique large homozygous regions on chromosome 3 and chromosome 10 and a few smaller homozygous regions on chromosomes 2, 5, 6, 12 and 13, for which the patient was homozygous and his parents heterozygous (Table 2). 
Table 2: Regions of homozygosity >1Mb identified by SNP array and WES data analysis in the patient.

\begin{tabular}{llll}
\hline Chromosome & Start position $(\mathrm{Mb})$ & End position $(\mathrm{Mb})$ & Megabases $(\mathrm{Mb})$ \\
\hline 2 & 105.7 & 108.6 & 3 \\
3 & 64.2 & 74.9 & 11 \\
3 & 192.6 & 195.4 & 3 \\
5 & 104.7 & 111.5 & 7 \\
6 & 36.6 & 42.2 & 6 \\
10 & 15.2 & 16.4 & 1 \\
10 & 71 & 85.6 & 13 \\
10 & 101.8 & 107.8 & 6 \\
12 & 128.4 & 129.5 & 1 \\
13 & 43.8 & 47.2 & 3 \\
\hline
\end{tabular}

Whole exome sequencing revealed a total of 57,267 variants. After filtering for presence in homozygous regions, frequency $<0.01$ in variation databases (dbSNP and ExAc), conservation and pathogenicity prediction, only four variants in four different genes remained, PDZD7 (NM_001195263.1: c.2319_2337del, chr10: 102770309), WDR36 (NM_139281.2: c.635A>C, chr5: 110436345), HKDC1 (NM_025130.3: c.1441 C>T, chr10: 71008355) and MED20 (NM_004275.4: c.233 T>C, chr6: 41877197). Of these, PDZD7 is linked to Usher syndrome and WDR36 is involved in T-cell activation and is highly coregulated with IL12. WDR36 variants were associated with polygenic forms of Glaucoma [4], therefore, it is unlikely that mutations in these two genes could cause the phenotype observed in our patient. The HKDC1 mutation (NM_025130.3; c.1441 C>T) results in a premature stop codon (R481X) leading to the loss of almost half of the protein or nonsense-mediated decay. No RNA of the patient was available to test this. HKDC1 belongs to the hexokinase family and shares approximately $70 \%$ sequence homology at both the nucleotide and amino acid sequence with HK1. Given that mutations in HK1 are associated with neurological phenotypes [5], HKDC1 is a plausible candidate gene. The mutation located in MED20 (NM_004275.4; c.233 T>C) changes the highly conserved leucine at position 78 to proline. The c.233 T>C MED20 mutation is predicted to be damaging by SIFT (score 0.002) and possibly damaging by PolyPhen2 (score 0.606) (Fig.1). Interestingly, a homozygous variant in MED20 has been reported previously in 2 sibs with infantile-onset basal ganglia degeneration and brain atrophy and cataract in one of them [6], making it another good candidate gene for our patient. The mutations in HKDC1 and MED2O were confirmed by Sanger sequencing and segregated with the phenotype in the family (data not shown).

\section{Morpholino-mediated knockdown of HKDC1}

The functional effect of the nonsense HKDC1 mutation was investigated in more detail, using morpholino (MO)-mediated knock down in zebrafish. The zebrafish HKDC1 gene 
contains 18 exons and shares $74.97 \%$ protein identity with the human HKDC1 gene (Fig.2). Since the c.1441 C>T HKDC1 mutation leads to a premature stop codon in exon 10, two different HKDC1 splice MOs were designed to target the exon9-intron9 (E9I9) and exon10-intron10 (E10I10) splice junction to create a similar HKDC1 defect in zebrafish. HKDC1 MOs were injected into oocytes at doses ranging from 0.5-6 ng $\mathrm{MO} / \mathrm{embryo}$ and RNA was harvested at 48- hour post-fertilization

RT-PCR was performed with primers positioned in exons 8 and 11 of HKDC1 (Fig. 3A). Sequencing of the RT-PCR product from control MO embryos confirmed a 711-bp fragment, consistent for normally spliced mRNA. E9I9 results in two aberrant cDNAs, one smaller and one larger compared to the control (Fig. 3B). Sequence analysis of the aberrant cDNAs revealed that the smaller fragment corresponds to a $123 \mathrm{bp}$ deletion, immediately upstream of the correct exon 9 splice donor, causing a 41-amino acid inframe deletion. The larger fragment contained an insertion of 85-bp of intron 9, giving a frameshift and a premature stop codon after 7 amino acids (p.Pro424Valinefs*7) (Fig. 3C). RT-PCR of the CDNA of E10I10 MO injected embryos revealed an approximately 100 bp larger fragment compared to control embryos (Fig. 3B). Sequence analysis of this fragment revealed that the E10I10 MO resulted in the inclusion of 111-bp of intron 10, resulting in an in-frame insertion of 37 amino acids (Fig. 3C).

Quantitative PCR analysis of total mRNA showed an 80\% decrease of HKDC1 mRNA for the embryos injected with the E9I9 MO compared to control MO-injected embryos (Fig.4). This massive decrease in HKDC1 expression was observed at all MO doses $(0.5,1$ or 2 ng E9I9 MO). In contrast, the expression of HKDC1 for the embryos injected with the E10I10 MO was unchanged compared to control MO-injected embryos (Fig.4). At a dose of 0.5ng, E9I9 MO-injected embryos appeared normal at 48hpf and 72hpf (Fig. 5). Injection of $1 \mathrm{ng}$ of E9I9 MO resulted in 92.1\% embryos with smaller heads and eyes and some embryos showed heart edema. Injection of $2 \mathrm{ng}$ of E9I9-MO resulted in more severe phenotypes including smaller heads, smaller eyes and bent tails and $20-30 \%$ embryos without anterior-posterior axis. Heart edema was frequently observed in most embryos (Fig.5 and 6). Interestingly, at doses ranging from $0.5 \mathrm{ng}$ to $2 \mathrm{ng}$, E10l10 MO did not cause any visible phenotypic abnormalities compared to control $\mathrm{MO}$ injected embryos. Survival rate was similar in both E9I9 and E10I10 injected embryos compared to control-MO-injected embryos at MO dose of 1 and $2 \mathrm{ng}$ (Fig. 6). Higher doses of E9I9 $\mathrm{MO}$ (4 ng and 6ng) and E10l10 MO (6ng) produced highly necrotic embryos. These results show that injection of HKDC1 E9I9 MO but not E10I10 MO results in a dosedependent phenotype (Fig.5, 6). 
(A)

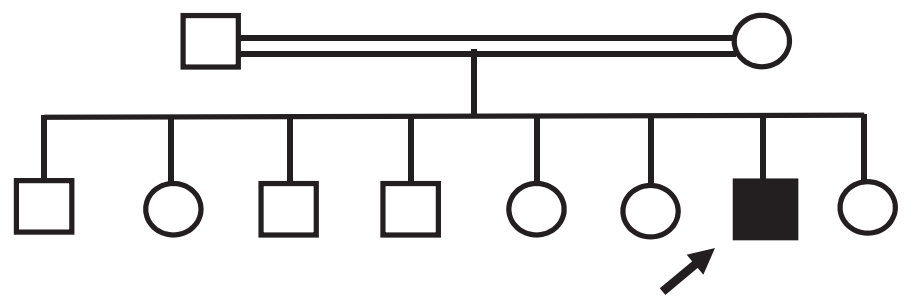

(B)
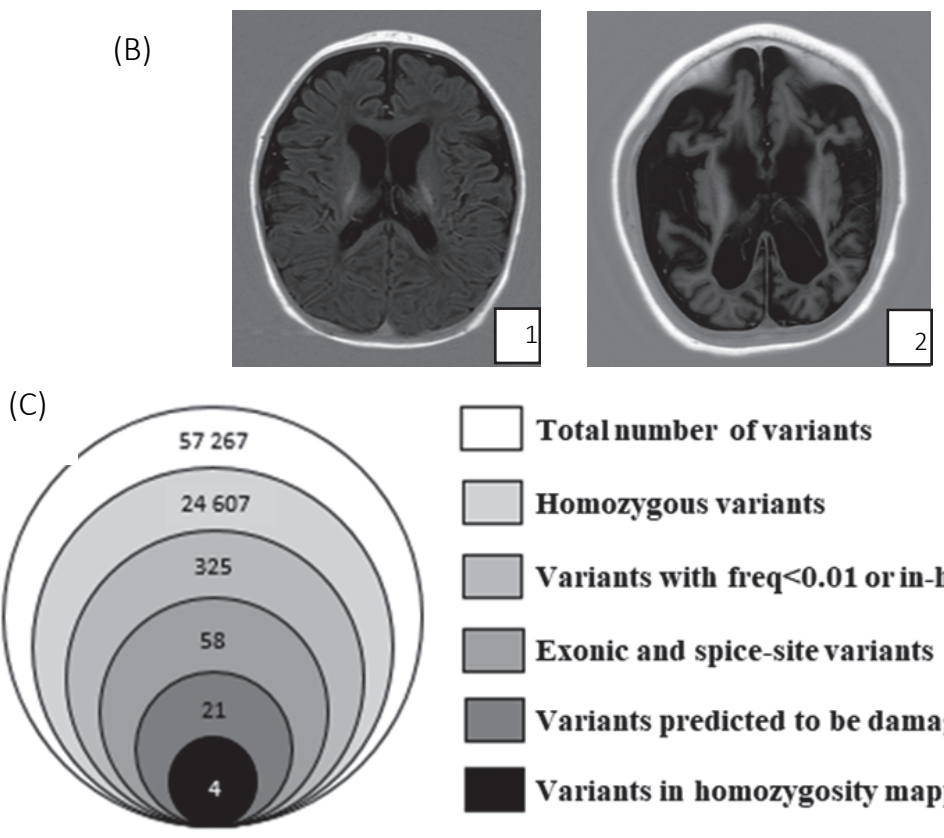

$\square$ Total number of variants

$\square$ Homozygous variants

\section{Variants with freq $<0.01$ or in-house freq $<0.01$}

Exonic and spice-site variants

Variants predicted to be damaging

Variants in homozygosity mapping

PDZD7 (NM_001195263.1: c.2319_2337del, chr10: 102770309)

WDR36 (NM_139281.2: c.635A $>$ C, chr5: 110436345)

HKDC1 (NM_025130.3: c.1441 C>T, chr10: 71008355)

MED20 (NM_004275.4: c.233 T>C, chr6: 41877197)

Fig. 1. MRI and exome data of the index patient. A) Pedigree of a consanguineous family with epilepsy, spasticity and brain atrophy (affected individuals indicated in black). The index patient is indicated with an arrow. B) brain magnetic resonance imaging at age 5 months (B1) and post mortem (B2) showing severe atrophy over time with both white and grey matter loss C) Schematic representation of the exome data, filtered for rare homozygous candidate variants. 


\begin{tabular}{|c|c|c|}
\hline $\begin{array}{l}\text { huran } \\
\text { zebrafish }\end{array}$ & 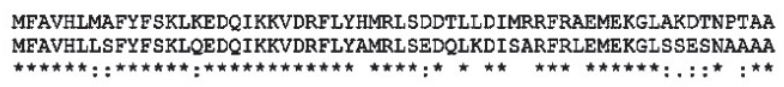 & $\begin{array}{l}60 \\
60\end{array}$ \\
\hline $\begin{array}{l}\text { human } \\
\text { zebrafish }\end{array}$ & 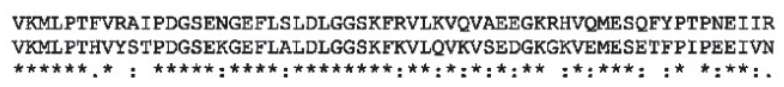 & $\begin{array}{l}120 \\
120\end{array}$ \\
\hline $\begin{array}{l}\text { human } \\
\text { zebrafish }\end{array}$ & 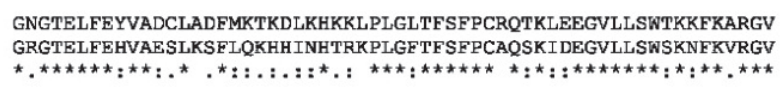 & $\begin{array}{l}180 \\
180\end{array}$ \\
\hline $\begin{array}{l}\text { human } \\
\text { zebrafish }\end{array}$ & 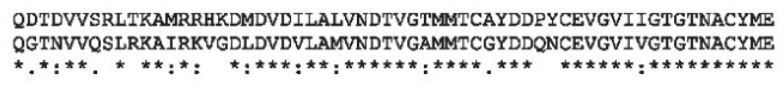 & $\begin{array}{l}240 \\
240\end{array}$ \\
\hline $\begin{array}{l}\text { human } \\
\text { zebrafish }\end{array}$ & 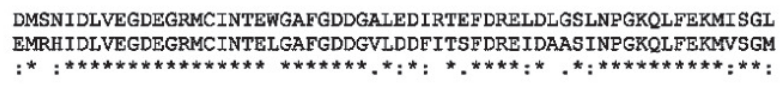 & $\begin{array}{l}300 \\
300\end{array}$ \\
\hline $\begin{array}{l}\text { human } \\
\text { zebrafish }\end{array}$ & 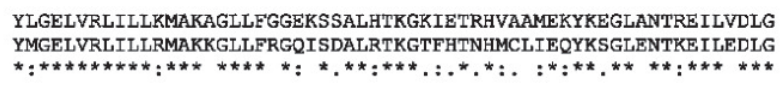 & $\begin{array}{l}360 \\
360\end{array}$ \\
\hline $\begin{array}{l}\text { human } \\
\text { zebrafish }\end{array}$ & 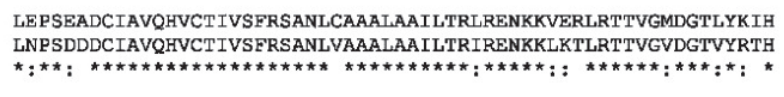 & $\begin{array}{l}420 \\
420\end{array}$ \\
\hline $\begin{array}{l}\text { human } \\
\text { zebrafish }\end{array}$ & 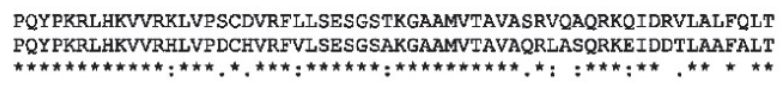 & $\begin{array}{l}480 \\
480\end{array}$ \\
\hline $\begin{array}{l}\text { human } \\
\text { zebrafish }\end{array}$ & 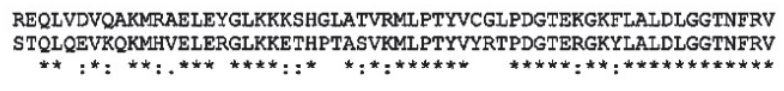 & $\begin{array}{l}540 \\
540\end{array}$ \\
\hline $\begin{array}{l}\text { human } \\
\text { zebrafish }\end{array}$ & 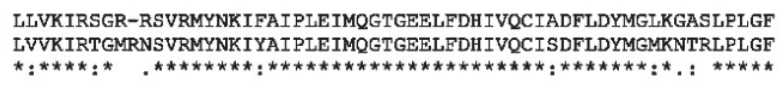 & $\begin{array}{l}599 \\
600\end{array}$ \\
\hline $\begin{array}{l}\text { human } \\
\text { zebrafish }\end{array}$ & 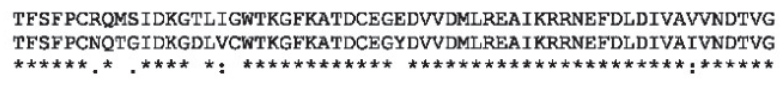 & $\begin{array}{l}659 \\
660\end{array}$ \\
\hline $\begin{array}{l}\text { human } \\
\text { zebrafish }\end{array}$ & 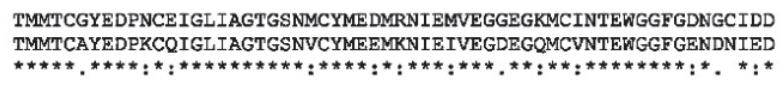 & $\begin{array}{l}719 \\
720\end{array}$ \\
\hline $\begin{array}{l}\text { human } \\
\text { zebrafish }\end{array}$ & 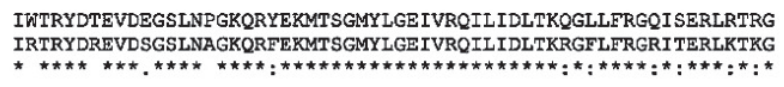 & $\begin{array}{l}779 \\
780\end{array}$ \\
\hline $\begin{array}{l}\text { human } \\
\text { zebrafish }\end{array}$ & 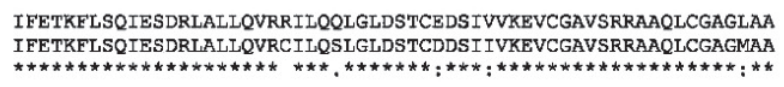 & $\begin{array}{l}839 \\
840\end{array}$ \\
\hline $\begin{array}{l}\text { human } \\
\text { zebrafish }\end{array}$ & 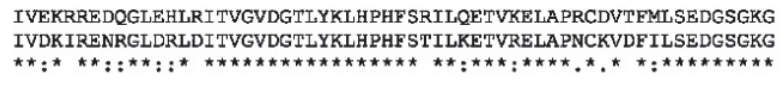 & $\begin{array}{l}899 \\
900\end{array}$ \\
\hline $\begin{array}{l}\text { human } \\
\text { zebrafish }\end{array}$ & $\begin{array}{l}\text { AALITAVAKRLQQAQKEN- } 917 \\
\text { AALITAVARQHHVQKQEQN } 919 \\
\star \star \star \star \star \star \star:: \quad::^{\star}:\end{array}$ & \\
\hline
\end{tabular}

Fig. 2: Alignment of the amino acid sequences of HKDC1 between human and zebrafish. Asterisk shows identical residues for $\mathrm{HKDC1}$; colon similar alternate residues; dot dissimilar alternate residues. Amino acid identity between the zebrafish and human HKDC1 is $74.97 \%$. 


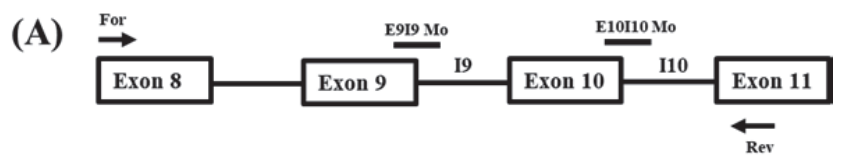

(B)

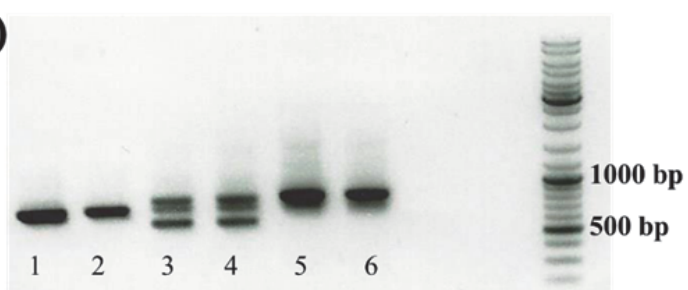

1: Control Morpholino $0.5 \mathrm{ng}$

2: Control Morpholino $1 \mathrm{ng}$

3: E9I9 Morpholino $0.5 \mathrm{ng}$

4: E9I9 Morpholino 1 ng

5: E10I10 Morpholino 0.5 ng

6: E10I10 Morpholino $1 \mathrm{ng}$

(C)

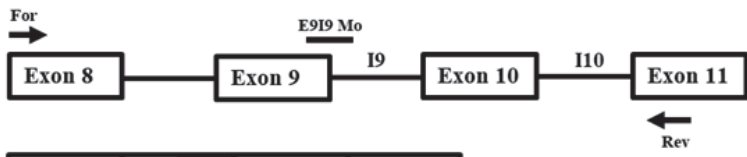

\begin{tabular}{|c|c|c|c|}
\hline Exon 8 & Exon 9 & Exon 10 & Exon 11 \\
\hline \\
$\begin{array}{c}123 \text { bp } \\
\text { deletion }\end{array}$
\end{tabular}

\begin{tabular}{|l|l|c|c|c|c|}
\hline Exon 8 & Exon 9 & 19 & Exon 10 & Exon 11 & Variant 2 \\
\cline { 3 - 4 } & 85 bp & Ex
\end{tabular}

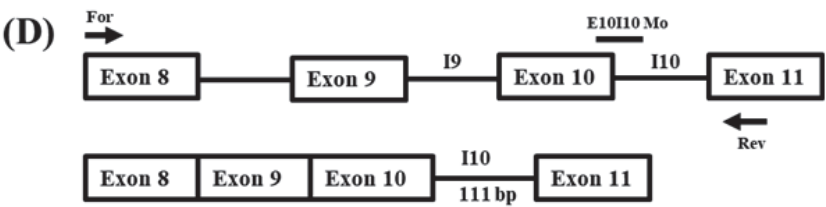

Fig. 3: Morpholinos efficiently block normal splicing of HKDC1 (A) The position of primers used to amplify spliced mRNA product (arrows) and morpholinos against exon-intron boundary (bold lines). (B) RT-PCR analysis of HKDC1 covering exon8-11 of mRNA extracted from embryos injected with control, E9I9 and E10I10 MOs demonstrated that the E9I9 and E10110 MOs lead to aberrant transcripts. (C, D) Schematic representation of the transcripts resulting from splicing defects due to E9I9 and E10I10 MOs. 


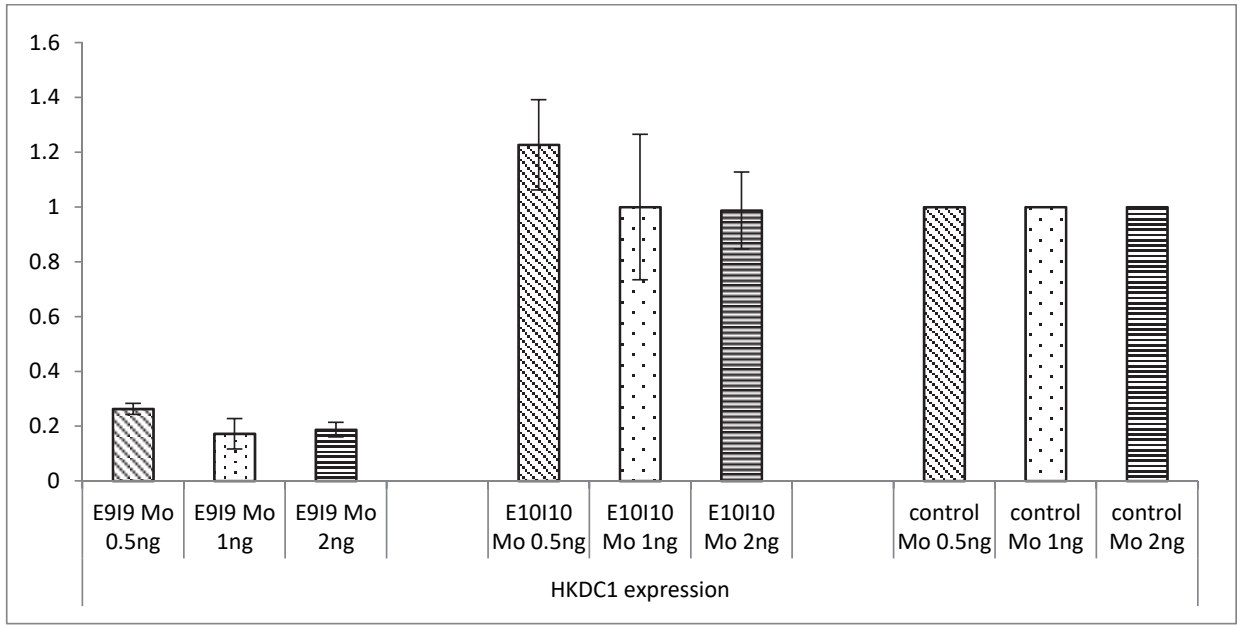

Fig. 4: HKDC1 expression in control and HKDC1 knockdown embryos. Quantitative RT-PCR for HKDC1 mRNA in morpholino injected embryos at 2 days post fertilization shows efficient knockdown of HKDC1 expression for the E9I9 MO but not the E10I10 MO. The Y axis shows for HKDC1 expression normalized first to ODC1 and then to Control Mo sample, valued as 1. Error bars are +/- SD
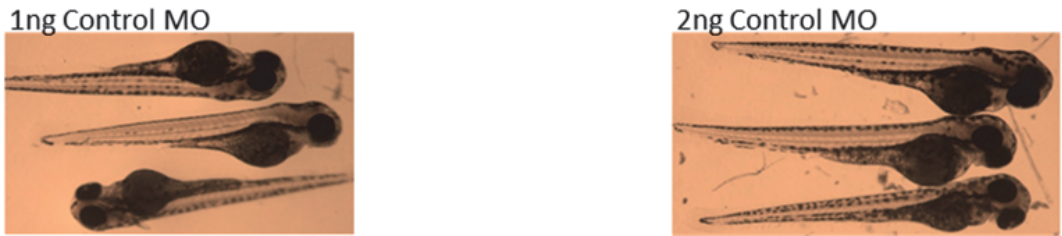

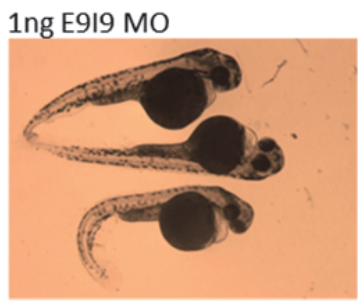

1ng E10I10 MO

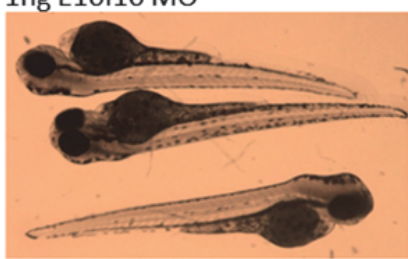

\section{2ng E9I9 MO}
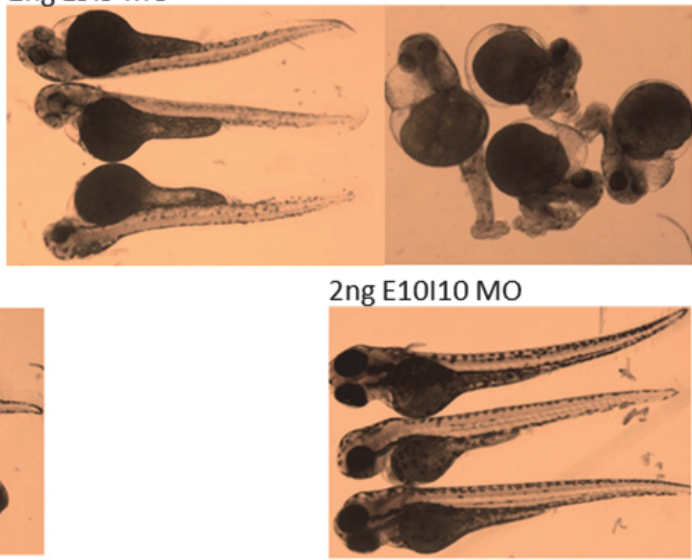

Fig. 5: Representative images of $72 \mathrm{hpf}$ embryos injected with control MO, E9I9 and E10I10 HKDC1 MOs. E9I9 HKDC1 morphants show severe phenotypes including reduced head, eyes size (1ng) and bent tails (2ng) whereas E10I10 HKDC1 morphants are similar to control injected embryos. 

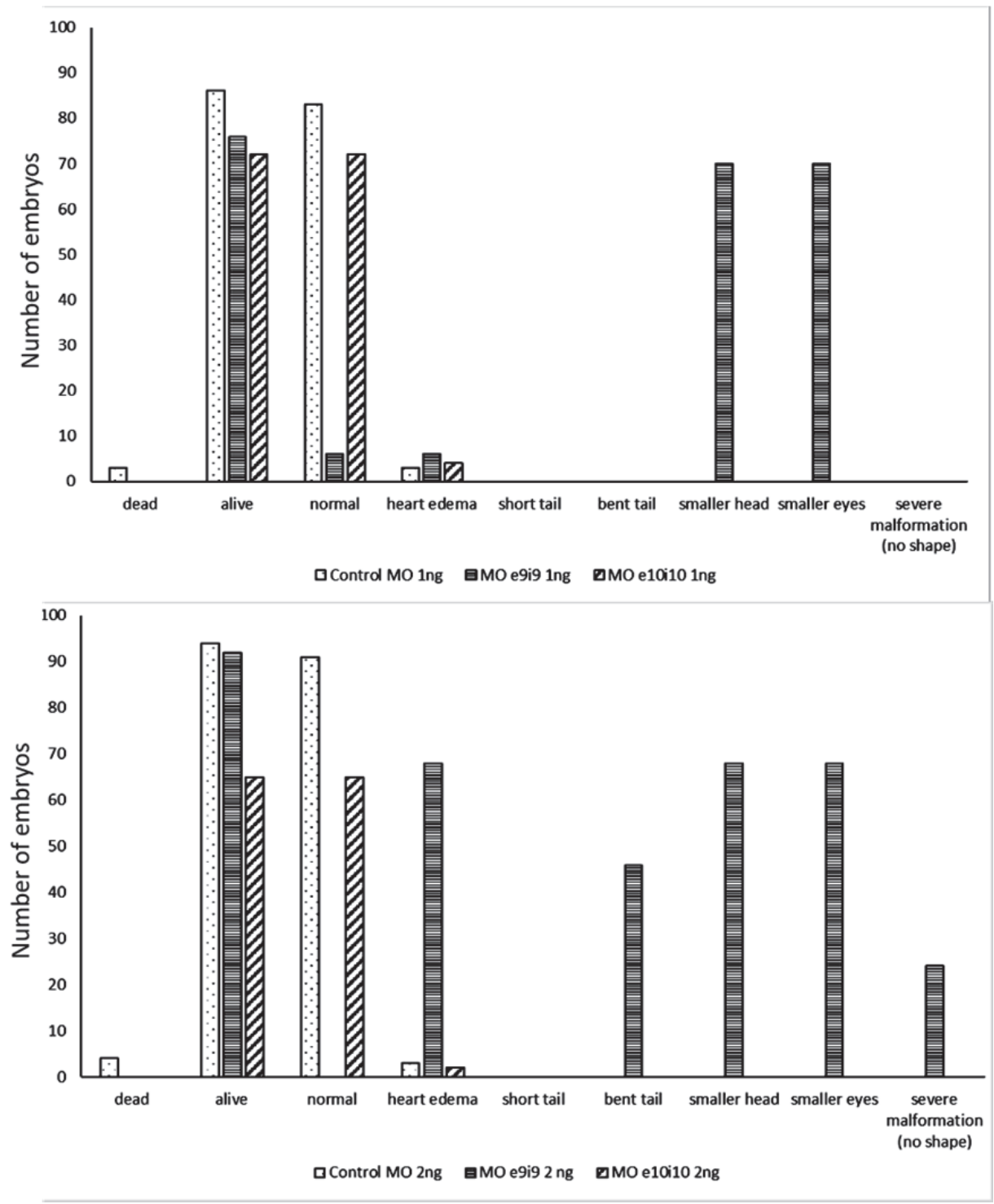

Fig. 6: Effect of HKDC1 knockdown in zebrafish embryos. Number of embryos showing dead, alive, normal, heart edema, short tail, bent tail, small head, small eye, and deformed phenotypes observed at $48 \mathrm{hpf}$ and 72 hpf at different doses of control MO, E9I9 and E10I10 HKDC1 MOs.

\section{DISCUSSION}

In a child of consanguineous parents with congenital cataract, severe psychomotor regression, epilepsy and secondary microcephaly, whole Exome Sequencing (WES) was 
performed combined with homozygosity mapping leading to the identification of homozygous mutations in MED2O and HKDC1, which were predicted to be damaging to the protein. MED20 encodes one of 25 subunits of the Mediator complex, a coactivator of RNA polymerase II transcribed genes, that interacts with DNA-bound transcriptional activators and general initiation factors. The mediator complex consists of 4 modules namely the head, middle, tail and kinase module. MED20 is one of 7 subunits that constitute the head module. The head module is organized into three domains, and interacts with RNA polymerase II and other transcription factors [7]. Mutations in the mediator complex have been associated with infantile cerebral and cerebellar atrophy (MED17) [8], Lujan-Fryns syndrome [9], Ohdo syndrome, X-linked [10], and OpitzKaveggia syndrome [11] (MED12), intellectual disability (MED23) [12], Charcot-MarieTooth Disease Type 2B2 (MED25) [13]. For MED20, only one homozygous, potentially damaging variant has been reported in two Australian siblings with infantile basal ganglia degeneration and brain atrophy [6]. One of these girls also showed cataract at the age of 11-years. The conclusions from this study are limited as the findings are gathered from only one family and based on prediction models only. As the age at onset and clinical symptoms of our patients are more severe, we investigated also a potential role of the HKDC1 mutation in the clinical manifestations.

$H K D C 1$ belongs to the hexokinase family and is broadly expressed with a high expression in brain [14]. Hexokinases are an evolutionarily conserved family of kinases which catalyze the phosphorylation of glucose during glycolysis [15]. HKDC1 shares approximately $70 \%$ sequence homology at both the nucleotide and amino acid sequence with HK1. The differential localization of HK1 and HKDC1 expression suggests that they have both shared and distinct roles in the brain [14]. Given that mutations in HK1 are associated with neurological phenotypes [5], HKDC1 is a highly plausible candidate gene in this study. Human HKDC1 shows $75 \%$ protein identity with zebrafish HKDC1. As the mutation in HKDC1 was a nonsense mutation, probably leading to nonsense-mediated decay, the phenotypic effect of defective HKDC1 was investigated using MO-mediated knock down in zebrafish. Morpholinos are an effective way of transiently knocking down zebrafish gene function [16]. HKDC1 consists of 18 exons in both humans and zebrafish. As the nonsense mutation (R481X) in HKDC1 resulted in a premature stop codon in exon 10, two morpholinos were created to target the exon9-intron9 and exon10-intron10 boundary in zebrafish. Injection of the E9I9 MO resulted in two aberrant transcripts creating a premature stop codon or in-frame deletion while the E10I10 MO led to the retention of intron 10. Quantitative PCR analysis demonstrated that the embryos injected with E9I9 MO barely had any HKDC1 mRNA, most likely due to nonsense-mediated decay (NMD). Phenotypically, more than 90\% of E9I9- HKDC1 morphants displayed small heads and eyes. In contrast, E10I10-injected embryos exhibited the wild-type phenotype indicating that a transcript with the insertion of 111-bp of intron 10 was translated and did not cause visible pathology. These results provide evidence that the phenotype caused by E9I9 MO is specific to the loss of HKDC1 func- 
tion. This indicate $H K D C 1$ could be involved in neurological development and/or brain functions and implicate HKDC1 mutations in the patient phenotypes. The results are supported by the previous publications characterizing zebrafish models of neurological diseases. Zebrafish displayed neurodegenerative phenotypes including small retina, small heads and curved bodies which correlated with CLN2 disease, a paediatric neurodegenerative disease with widespread brain atrophy, and profound neuron loss within the retina and brain [17]. In another zebrafish modeling Pontocerebellar hypoplasia type 7 (PCH7), neurodegeneration and ambiguous genitalia, zebrafish had midbrain and hindbrain degeneration resembling PCH7 disease [18].

Our findings showed WES as a powerful tool for identifying gene defects that were implicated in this patient and in neurodegenerative disorders in general. WES has been used the last couple of years with success to identify known and novel gene defects in individuals with neurodegenerative diseases $[6,19,20]$. The sensitivity, speed and cost make WES the preferred method. It is increasingly being used, even for large-scale population studies. However, WES also has its limitations, as exemplified by cases in which the genetic causes could not be detected. This could be due to incomplete or low coverage of certain exons or even whole genes, extreme GC-rich regions, mutations within intronic regions, and interactions between two or more genes that may cause disease. WES enabled us to identify two homozygous mutations. Of those, HKDC1 was a novel candidate gene. Based on our data we cannot draw a definite conclusion on the role of MED2O and HKDC1 in the pathology of our patient. For different reasons, both of them are good candidates, and even a synergistic role for both genes is a possibility. Follow-up investigations in the family or in the region from which the family originated, might provide the solution. While seven siblings of the patient were healthy, six siblings from the mother had deceased at the age of 10 days-18 months having anamnestically a similar phenotype, although no objective records exist. Family history showed that the mother originated from a consanguineous marriage. Unfortunately, no material and clinical data were available from the mother's siblings in Syria, but studies in that region might reveal other patients with the same phenotype and could identify one of the mutations or both as founder mutations. Additional functional studies could also help define the exact role of both genes in the phenotype and to elucidate the pathophysiological mechanism. This would require a model which would have the same phenotypic manifestation as the patient. In conclusion, we demonstrated that the importance to perform a complete exome analysis to identify the underlying genetic cause of disease. Although we cannot draw a definite conclusion in our patient yet, one should not stop when a possible candidate gene defect has been identified as this might lead to missing other candidates, which could be the real cause. 


\section{REFERENCES}

1. Woods, C.G. and A. Parker, Investigating microcephaly. Arch Dis Child, 2013. 98(9): p. 707-13.

2. Gerards, M., et al., Exome sequencing reveals a novel Moroccan founder mutation in SLC19A3 as a new cause of early-childhood fatal Leigh syndrome. Brain, 2013. 136(Pt 3): p. 882-90.

3. Nguyen, M., et al., Pathogenic CWF19L1 variants as a novel cause of autosomal recessive cerebellar ataxia and atrophy. Eur J Hum Genet, 2016. 24(4): p. 619-22.

4. Skarie, J.M. and B.A. Link, The primary open-angle glaucoma gene WDR36 functions in ribosomal RNA processing and interacts with the p53 stress-response pathway. Hum Mol Genet, 2008. 17(16): p. 247485.

5. Sevilla, T., et al., Genetics of the Charcot-Marie-Tooth disease in the Spanish Gypsy population: the hereditary motor and sensory neuropathy-Russe in depth. Clin Genet, 2013. 83(6): p. 565-70.

6. Vodopiutz, J., et al., MED20 mutation associated with infantile basal ganglia degeneration and brain atrophy. Eur J Pediatr, 2015. 174(1): p. 113-8.

7. Imasaki, T., et al., Architecture of the Mediator head module. Nature, 2011. 475(7355): p. 240-3.

8. Kaufmann, R., et al., Infantile cerebral and cerebellar atrophy is associated with a mutation in the MED17 subunit of the transcription preinitiation mediator complex. Am J Hum Genet, 2010. 87(5): p. 667-70.

9. Schwartz, C.E., et al., The original Lujan syndrome family has a novel missense mutation (p.N1007S) in the MED12 gene. J Med Genet, 2007. 44(7): p. 472-7.

10. Vulto-van Silfhout, A.T., et al., Mutations in MED12 cause X-linked Ohdo syndrome. Am J Hum Genet, 2013. 92(3): p. 401-6.

11. Risheg, $H_{\text {., }}$ et al., A recurrent mutation in MED12 leading to R961W causes Opitz-Kaveggia syndrome. Nat Genet, 2007. 39(4): p. 451-3.

12. Hashimoto, S., et al., MED23 mutation links intellectual disability to dysregulation of immediate early gene expression. Science, 2011. 333(6046): p. 1161-3.

13. Leal, A., et al., Identification of the variant Ala335Val of MED25 as responsible for CMT2B2: molecular data, functional studies of the SH3 recognition motif and correlation between wild-type MED25 and PMP22 RNA levels in CMT1A animal models. Neurogenetics, 2009. 10(4): p. 275-87.

14. Ludvik, A.E., et al., HKDC1 Is a Novel Hexokinase Involved in Whole-Body Glucose Use. Endocrinology, 2016. 157(9): p. 3452-61.

15. Wilson, J.E., Isozymes of mammalian hexokinase: structure, subcellular localization and metabolic function. J Exp Biol, 2003. 206(Pt 12): p. 2049-57.

16. Nasevicius, A. and S.C. Ekker, Effective targeted gene 'knockdown' in zebrafish. Nat Genet, 2000. 26(2): p. 216-20.

17. Mahmood, F., et al., A zebrafish model of CLN2 disease is deficient in tripeptidyl peptidase 1 and displays progressive neurodegeneration accompanied by a reduction in proliferation. Brain, 2013. 136(Pt 5): p. 1488-507.

18. Lardelli, R.M., et al., Biallelic mutations in the 3' exonuclease TOE1 cause pontocerebellar hypoplasia and uncover a role in snRNA processing. Nat Genet, 2017. 49(3): p. 457-464.

19. O'Brien, A., et al., Severe neurodegeneration, progressive cerebral volume loss and diffuse hypomyelination associated with a homozygous frameshift mutation in CSTB. Eur J Hum Genet, 2017. 25(6): p. 775-778.

20. Megahed, H., et al., Utility of whole exome sequencing for the early diagnosis of pediatric-onset cerebellar atrophy associated with developmental delay in an inbred population. Orphanet J Rare Dis, 2016. 11(1): p. 57 



\section{Chapter}

\section{Whole exome sequencing is the preferred, first strategy to identify known and novel causes of mitochondrial disease}

Tom E.J. Theunissen, Minh Nguyen, Rick Kamps, Alexandra T. Hendrickx, Suzanne C.E.H. Sallevelt, Ralph W.H. Gottschalk, Chantal M. Calis, Alphons P.M. Stassen, Bart de Koning, Elvira N. M. Mulder-Den Hartog, Kees Schoonderwoerd, Jo Vanoevelen, Radek Szklarczyk, Mike Gerards, Irenaeus F.M. de Coo*, Debby M.E.I. Hellebrekers*, Hubert J.M. Smeets*

* These authors contributed equally to this work.

Manuscript submitted 


\section{ABSTRACT}

Mitochondrial diseases are clinically and genetically heterogeneous and can be caused by both mitochondrial DNA (mtDNA) and nuclear genetic defects. We have performed a two-step next-generation sequencing-based approach in a cohort of 118 patients, mostly children, with either a high suspicion of a mitochondrial disorder or with a disease phenotype in which mitochondrial defects are part of the differential diagnosis. Patients were screened for mtDNA defects and, if excluded, analysed by whole exome sequencing (WES). We filtered for autosomal recessive and $\mathrm{X}$-linked recessive mutations in families with multiple affected siblings and/or consanguineous parents. Nonconsanguineous families with a single patient were additionally screened for autosomal and X-linked dominant mutations in a predefined gene set. We detected and quantified mtDNA point-mutations and indels in $20 \%$ of the patient-cohort, and identified diseasecausing exonic mutations in $49 \%$ of the patients, implying an overall diagnostic yield of $69 \%$. We show that $32 \%$ of the disease-causing genes in mitochondrial WES-patients were not in the MitoCarta database at the time of discovery, and therefore would have been missed with targeted sequencing approaches. WES is a comprehensive and unbiased approach to establish a genetic diagnosis in mitochondrial disease and able to fully resolve heterogeneous and multi-genic disease manifestations. 


\section{INTRODUCTION}

Mitochondrial disorders are most often characterized by deficiencies in oxidative phosphorylation (OXPHOS) and ATP production, and manifest with a broad spectrum of clinical symptoms. A single gene defect can demonstrate a broad and complex variety of symptoms (clinical heterogeneity) and many different gene defects can result in a similar phenotype (genetic heterogeneity). Characterization of the genetic basis of mitochondrial disease is further complicated due to the involvement of both the nuclear and mitochondrial genomes[1, 2]. For pathogenic mutations in the multi-copy mtDNA, which contains 37 genes and is exclusively maternally inherited, the percentage of socalled heteroplasmic mutations also affects the clinical presentation[3, 4]. The estimated number of nuclear genes involved in mitochondrial function is around $1500[5,6]$, of which only $>250$ genes have been shown to be involved in mitochondrial disease[7]. In primary mitochondrial disorders (MD) the OXPHOS deficiencies are often due to genetic defects in the OXPHOS complexes (subunits and assembly factors), or, more indirectly, in processes such as mitochondrial protein translation and degradation, mtDNA maintenance, fusion and fission, substrate transport or phospholipid metabolism. Still, mitochondrial dysfunction can also be a more secondary defect in genetic syndromes or neuromuscular disease[8]. Finally, for a broad variety of clinical entities, a mitochondrial gene defect can explain a minority of cases. These were analyzed as well by WES and referred to as differential diagnosis (DD).

Genetic diagnosis of mtDNA-disorders requires screening of all 37 mtDNA genes, determining heteroplasmy levels of variants and characterizing large mtDNA deletions and mtDNA copy number. mtDNA analysis in diagnostic setting commonly started with screening for a few relatively common point mutations using mutation-specific restriction enzymes or qPCR based methods[9, 10]. In case common mutations were not detected, the entire mitochondrial genome was analysed by Sanger sequencing or chipbased methods[11, 12]. However, these methods are non-quantitative, requiring a second molecular test to determine the mutation load. Besides, chip-based methods have difficulties in detecting small indels[13-15]. For the large mtDNA deletions, it was time-consuming to determine the exact breakpoints, which could be important for prognosis[16, 17]. The application of next-generation sequencing has greatly increased the possibilities for detecting and quantifying point mutations and indels across the complete mitochondrial genome and for characterizing large mtDNA deletions with one single technology[18-20]. Although, accurate determination of the heteroplasmy levels of large mtDNA deletions and mtDNA copy number still require quantitative PCR analysis. If no mtDNA mutations are present, then moving into the analysis of nuclear genes traditionally relied on (stepwise) Sanger sequencing of nuclear candidate genes which were selected based on clinical and biochemical features or linkage/homozygosity mapping. Development of next-generation sequencing methods, such as WES, enable the detection of the majority of the genetic variations in the coding part of the genome[21]. 
An unbiased and complete genetic analysis is important, especially in heterogeneous mitochondrial disease, where genotype-phenotype relations can be indistinct and novel genes involved.

We have performed a complete next-generation sequencing strategy, analysing the mtDNA and exome in a cohort of 85 patients with primary mitochondrial disease and 33 patients where a mitochondrial cause is part of the differential diagnosis. The results were obtained over a 4-year period from 2012 to 2016. Patients were tested for mtDNA mutations by next-generation sequencing and, if negative, further analysed by WES. WES-variants were filtered according to the presumed genetic model of disease inheritance, allele frequencies and the effect and conservation of the variant. With this approach, we identified in about $70 \%$ of our patients a causative mutation in new and known disease genes, which were either inherited or de novo.

\section{MATERIAL AND METHODS}

\subsection{Patients}

118 consanguineous and non-consanguineous patients in this study were under treatment at Erasmus MC, Maastricht UMC+, Leiden UMC, UMC Utrecht, AMC Amsterdam (The Netherlands), and informed consent was obtained for WES analysis. Patients were classified as having a "mitochondrial disorder" (MD) if their profile met clinical criteria and/or biochemical/genetic criteria. Patients not meeting these criteria were part of the differential diagnostic (DD) procedure. MD is based on:

- Clinical criteria: Mitochondrial syndromes (LHON, CPEO/KSS, MERRF, MIDD/MELAS, Leigh syndrome, Miller- and Barth-syndrome). Patients were considered mitochondrial, if symptom profiles were scored as "probably" (5-7 points) or "definite" (8-12 points) mitochondrial disease, according to the "Nijmegen Clinical Criteria for Mitochondrial Disease".

- Biochemical criteria: A decreased mitochondrial respiratory capacity in muscle tissue or fibroblasts, measured as significantly decreased OXPHOS complex activity (isolated or combined OXPHOS deficiencies), reduced oxygen consumption, and/or decreased ATP production.

- Genetic criteria: Presence of mtDNA depletion or multiple mtDNA deletions

\section{2 mtDNA analysis}

Sequencing of the mtDNA was performed using the Illumina MiSeq platform. Enrichment of the entire mtDNA was performed by a single long-range PCR using Phusion Hot Start DNA polymerase II kit (Thermoscientific) and 100 ng of total genomic DNA, according to the manufacturer's instructions. Library preparation was performed by the Illu- 
mina Nextera XT kit according to the manufacturer's instructions. Twelve indexed DNA libraries were equimolarly pooled and sequenced in a single lane of $1 \mathrm{MiSeq}$ flow-cell with a 2x300bp paired-end chemistry. Data demultiplexing was performed with Illumina CASAVA software (v.1.8.4.) and reads were aligned against the revised Cambridge mitochondrial reference sequence (but without the gap at position 3107) by BWA software (v.0.5.9.)[22]. For both variant and small indel identification, Python 2.6.6., Python package pysam 0.7.8. and SAMTools 0.1 .19 software were used[23]. Large deletions were identified by alignment with the Smith and Waterman algorithm and the EMBOSS water program (v.6.5.7.). Annotation and filtering of mtDNA variants and indels were performed using in-house build Perl tools and a MySQL annotation database. Calculation of the heteroplasmy level at any nucleotide position was performed by the read depth of the mutant versus reference nucleotide. We have validated the detection and quantification accuracy of our NGS strategy by analysing DNA samples with varying heteroplasmy levels of different substitution mutations, small indels and large deletions, as previously determined by MitoChip, RFLP and PCR/Southern blotting (data available on request). The entire mtDNA, excluding the highly polymorphic D-loop, was analysed using a $2 \%$ heteroplasmy cut-off for known pathogenic point mutations and a $5 \%$ cut-off for the remaining positions and small indels. Heteroplasmy levels of large mtDNA deletions were determined by qPCR, using SensiMix SYBR (Bioline). Also, to identify mtDNA depletion in muscle biopsies (if available), qPCR quantification was performed, where mtDNA copy-number (based on MT-ND1) was normalized to the nDNA copy-number (based on B2M).

\subsection{Homozygosity Mapping and WES}

Homozygosity mapping and CNV analysis was performed by HumanMapping 250K array (Affymetrix, Santa Clara, California) and Genotyping console 4.0 (Affymetrix). Homozygosity or hemizygosity regions were defined by the "Homozygosity" mapper[24], with a cutoff of $5 \mathrm{MB}$. Exons were captured with SureSelect version 5 exome enrichment kit (Agilent Technologies, Santa Clara, California), including untranslated regions. Sequencing was performed on a HiSeq2000 platform (Illumina, San Diego, California), using a $2 \times$ 100bp paired-end setting. Bcl2fastq 1.8.4 (Illumina) allowed Basecalling and demultiplexing, and Burrows-Wheeler Aligner 0.5.9 (Broad Institute, Cambridge, Massachusetts) was used for read alignment against human reference genome hg19. Duplicate reads were removed by Picard software suite 1.77 (Broad Institute, Cambridge, Massachusetts) and variant calling was performed with GATK 2.1-8 (Broad Institute).

Exome data of consanguineous families or families consisting of $>1$ patient was filtered for recessive homozygous, compound heterozygous and X-linked (XLR) mutations. Variants with allele frequencies $<1 \%$ (dbSNP137, Exome Aggregation Consortium) were evaluated, covering missense mutations, indels, nonsense mutations, and splice variants. Non-annotated variants were maintained, unless allele frequencies exceeded $5 \%$ 
prevalence in our in-house patient database. Pathogenicity of nonsynonymous missense mutation was estimated by Polymorphism Phenotyping-2 (PolyPhen-2; Harvard, Boston, Massachusetts), Sorting Intolerant From Tolerant (J. Craig Venter Institute, Rockville, Maryland), Protein Variation Effect Analyzer (PROVEAN; J. Craig Venter Institute), and MutationTaster (NeuroCure Cluster of Excellence/Berlin Institute of Health, Berlin, Germany). Nonsense, frameshift, and splice-site variations were maintained. WES data of single non-consanguineous patients were additionally filtered for heterozygous mutations in known (OMIM (Online Mendelian Inheritance in Man) disease genes with known dominant and/or de novo mutations. All identified pathogenic mutations were checked for their inclusion in the MitoCarta database for mitochondrial localized proteins.

\section{RESULTS}

\subsection{Diagnostic yield}

A cohort of $118 \mathrm{MD}$ and DD index-patients from unaffected parents, was subject to a two-step next-generation sequencing approach (Figure 1). The age of disease onset in the cohort varied from birth to approximately 50 years old, 78\% (92/118) were pediatric patients ( $<18$ years of age). We solved $20 \%$ of the cohort with a disease-causing mtDNA defect, involving $24 \mathrm{MD}$-patients. Subsequent WES analysis solved an additional 49\% (58 patients) of the cohort, consisting of $38 \mathrm{MD}$-patients and 20 DD-patients. With this strategy, we achieved an overall diagnostic yield of $69 \%$, with comparable yield in the group of inherited disease cases (71\%) compared to the single non-consanguineous patients (68\%). 


\begin{tabular}{|c|c|c|c|}
\hline \multicolumn{4}{|c|}{118 patients } \\
\hline $85 \mathrm{MD}$ patients & \multicolumn{3}{|c|}{33 DD patients } \\
\hline \multicolumn{4}{|c|}{ mtDNA NGS: $20 \%$ solved } \\
\hline $24 \mathrm{MD}$ patients & \multicolumn{3}{|c|}{0 DD patients } \\
\hline \multicolumn{4}{|c|}{ WES: $49 \%$ solved } \\
\hline $38 \mathrm{MD}$ patients & \multicolumn{3}{|c|}{$20 \mathrm{DD}$ patients } \\
\hline \multicolumn{4}{|c|}{$69 \%$ diagnostic yield (82 patients) } \\
\hline family & total & MD & DD \\
\hline Consanguineous, $>1$ patient & $71 \%(32 / 45)$ & $71 \%(22 / 31)$ & $71 \%(10 / 14)$ \\
\hline Non-consanguineous, 1 patient & $68 \%(50 / 73)$ & $74 \%(40 / 54)$ & $53 \%(10 / 19)$ \\
\hline
\end{tabular}

Figure 1: The diagnostic yield of mtDNA and whole exome sequencing in a patient cohort consisting of $118 \mathrm{MD}$ and DD patients. $20 \%$ of the patients were solved with a mtDNA defect and $49 \%$ with a nuclear DNA defect, implying an overall diagnostic yield of $69 \%$.

\section{mtDNA next-generation sequencing}

mtDNA sequencing resulted in the identification of a disease-causing mutation in $20 \%$ $(24 / 118)$ of the patient cohort (Figure 1$)$, solving $4 \%(2 / 45)$ of the inherited disease cases (consanguineous, $>1$ patient) and 30\% (22/73) of the single non-consanguineous patients. As shown in Table 1, all cases with a mtDNA defect were MD-patients and all 24 identified mtDNA defects involved known mtDNA mutations that have been reported in LHON (Leber's hereditary optic neuropathy), MELAS (mitochondrial encephalomyopathy, lactic acidosis, and stroke-like episodes)/MIDD (maternally inherited diabetes mellitus and deafness), MERRF (myoclonic epilepsy with ragged red 
fibers), Leigh syndrome and CPEO (chronic progressive external ophthalmoplegia) / Kearns-Sayre syndrome. 38\% (9/24) of the mtDNA mutations were primary, mostly (8 out of 9) homoplasmic, LHON mutations (m.11778G $>$ A and m.14484T $>$ C), clinically manifesting with optic atrophy. Only one LHON patient carried $80 \%$ heteroplasmy. The m.3243A>G mutation covered 29\% (7/24) of the mtDNA defects, and explained both MELAS (2 patients) and MIDD (5 patients) phenotypes. Other mutations were identified in the ND5 gene (m.13513G>A) and the tRNA-Lysine gene (m.8344A>G), as a cause of Leigh syndrome and MERRF, respectively. 25\% (6/24) of the mtDNA defects were large single deletions, of which the 4977bp deletion (breakpoints 8482:13460) was detected in 4 patients. These patients had CPEO, sometimes in combination with additional symptoms of Kearns-Sayre syndrome (pigmentary retinopathy, cardiac conduction abnormalities). Patients with multiple mtDNA deletions (not included in Table 1) were further analysed by WES, as being suggestive of a underlying nuclear DNA defect in the mtDNA maintanance or replication genes. 
Whole exome sequencing is the preferred, first strategy to identify known and novel causes of mitochondrial disease

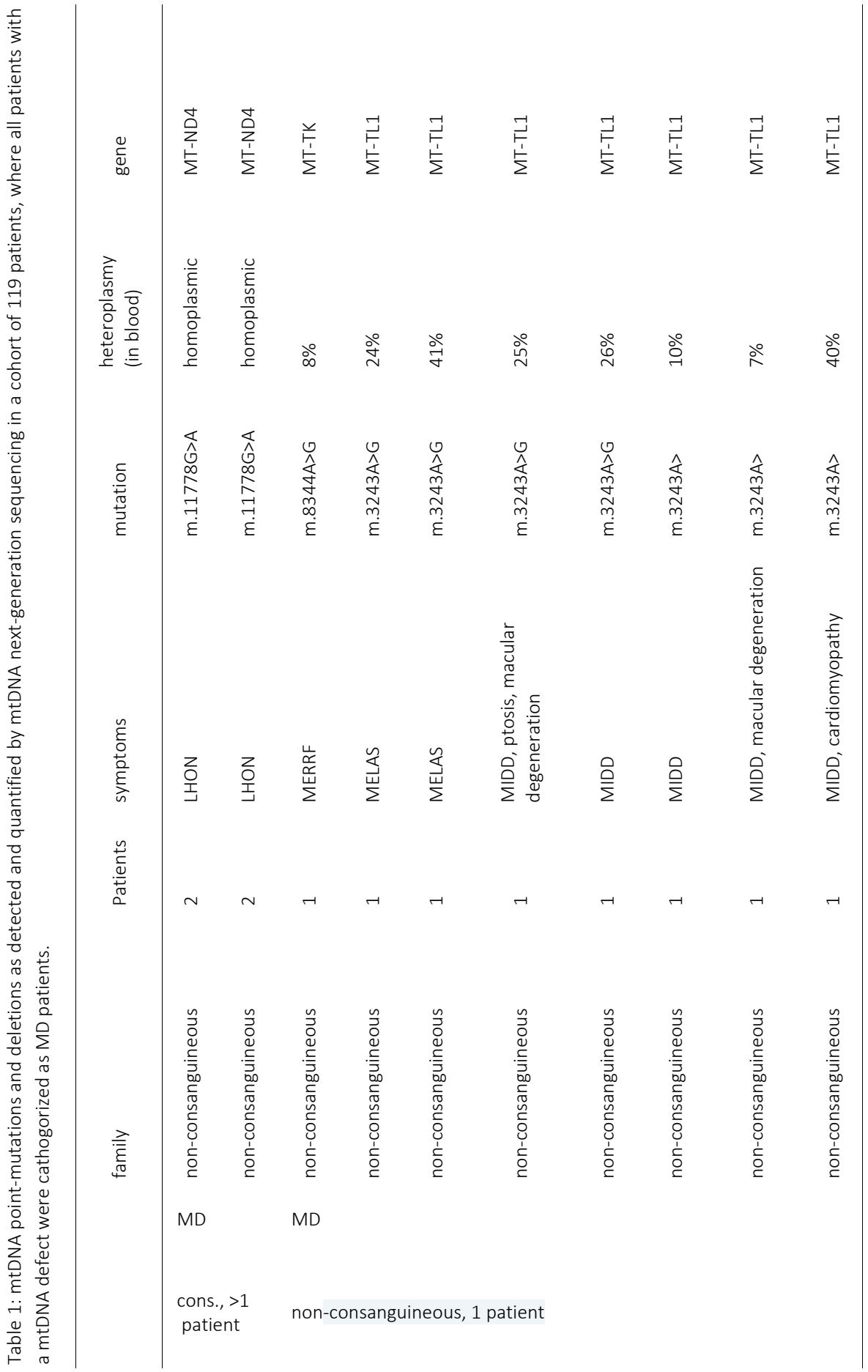




\section{Chapter 6}

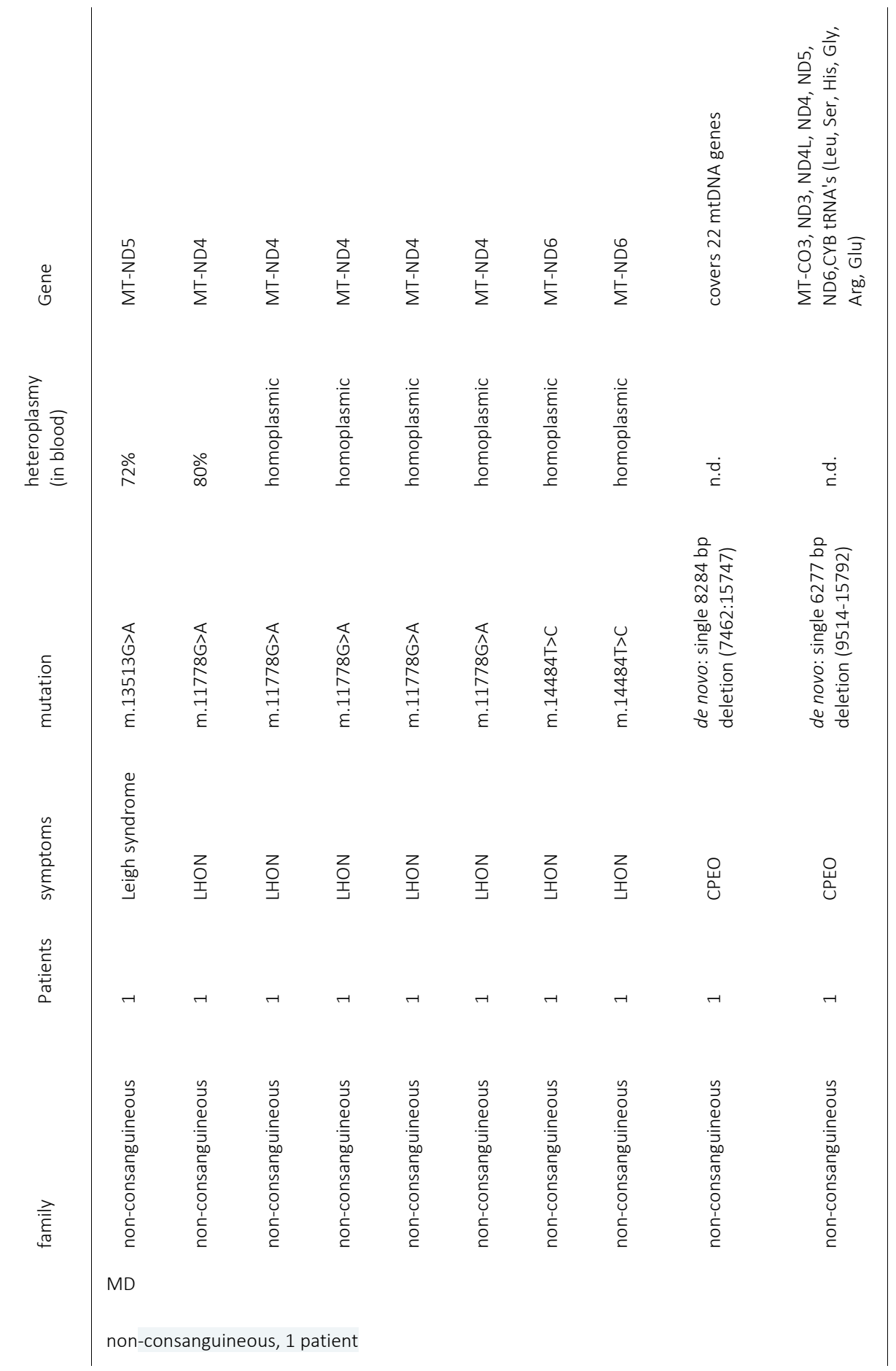


Whole exome sequencing is the preferred, first strategy to identify known and novel causes of mitochondrial disease

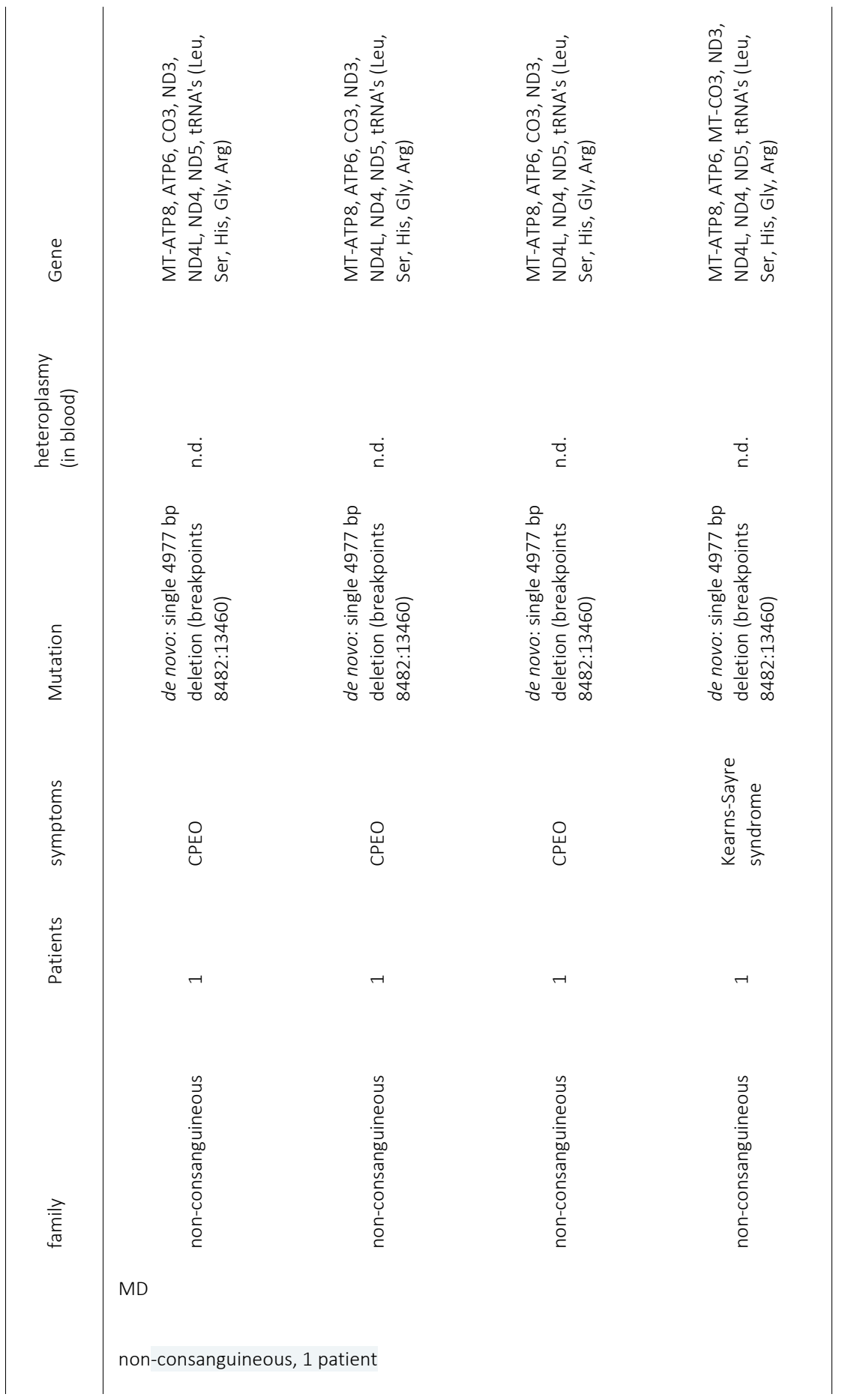




\section{WHOLE EXOME SEQUENCING}

In the remaining $94 \mathrm{MD}$ and DD patients, the presence of pathogenic mtDNA mutations was excluded. Prior to whole exome analysis, these patients were subject to SNP-array analysis, using the Affymetrix GeneChip Human Mapping 250K, to detect copy number variations (CNV) and homozygosity regions. No CNVs were detected. In consanguineous patients, mapping of the homozygous regions provided an additional filtering criterion to select the most promising genetic variants from the exome data. When multiple affected siblings were present, the entire family, if available, was analysed by means of SNP-arrays. This approach significantly increased the efficiency of interpreting WES results. WES identified a disease-causing gene defect in 49\% (58/118) of the complete patient cohort (Figure 1). In an additional $7 \%$ of the cohort, WES analysis identified a genetic variant which could explain the patient's phenotype, but for which definite evidence is currently lacking. Further studies should reveal which of these mutations could be classified as disease-causing.

WES-data filtering was based on the presumed genetic mode of inheritance. We applied a variant selection strategy for autosomal recessive (AR) and recessive $X$-linked $(\mathrm{XLR})$ disorders to the inherited disease cases (consanguinity, $>1$ patient). As shown in Table 2, this group consisted of 43 families, where WES identified of a genetic defect in $70 \%(30 / 43)$ of the cases, covering homozygous, compound heterozygous and X-linked mutations (Table 3). In MD patients, 69\% (20/29) of the cases were solved, and in the DD group a disease causing gene defect was found in $71 \%(10 / 14)$. A broad spectrum of genes with relatively few primary mitochondrial genes $(10 \%(1 / 10))$ was identified among the DD patients compared to the proportion of primary mitochondrial genes in MD patients (73\% (16/22)) (Table 3). In 51 non-consanguineous families with a single patient, we applied a variant selection strategy for autosomal recessive (AR), X-linked (XLR and XLD) and single heterozygous mutations, expected to be dominant de novo mutations. In order to limit the huge amount of heterozygous variants for dominant disease-causing mutations, variant interpretation was restricted to genes that have previously been reported in dominant disease based on the OMIM database. With this approach, we unravelled the genetic cause in 55\% (28/51) of these families (Table 2), $16 \%$ of which were single dominant mutations. Follow-up investigations in the parents revealed that the mutations either occurred de novo in the patients or were present in one of the parent, who displayed subclinical symptoms upon further investigation. The diagnostic yield was $56 \%(18 / 32)$ in the MD patient group and $53 \%(10 / 19)$ in the DD patient group, where respectively, $89 \%(17 / 19)$ and $18 \%(2 / 11)$ of the defects were located in a gene with reported mitochondrial function (Table 3 ). 
Table 2: 94 patients were subject to WES analysis. WES-data was filtered according to the presumed pattern of disease inheritance, where consanguinity or involvement of $>1$ patient were indicative of a recessively inherited disorder and non-consanguineous families with a single patient were likely to cover both, recessively inherited and dominant de novo mutations.

\begin{tabular}{|c|c|c|c|}
\hline WES filtering strategy & $\begin{array}{l}\text { Total } \\
\% \text { solved }\end{array}$ & $\begin{array}{l}\text { MD } \\
\% \text { solved }\end{array}$ & $\begin{array}{l}\text { DD } \\
\% \text { solved }\end{array}$ \\
\hline $\begin{array}{l}\text { consanguineous and/or }>1 \text { patie } \\
-\quad \text { autosomal recessive (AR) } \\
-\quad \text { X-linked (XLR) }\end{array}$ & $70 \%(30 / 43)$ & $69 \%(20 / 29)$ & $71 \%(10 / 14)$ \\
\hline $\begin{array}{ll}\text { non-consanguineous, } 1 \text { patient } \\
-\quad \text { autosomal recessive (AR) } \\
\text { - } \quad \text { X-linked (XLR and XLD) } \\
\text { - } \quad \text { autosomal dominant (AD) }\end{array}$ & $\begin{array}{l}55 \%(28 / 51) \\
\text { of which } 16 \% \text { de novo } \\
(8 / 51)\end{array}$ & $56 \%(18 / 32)$ & $53 \%(10 / 19)$ \\
\hline
\end{tabular}

Overall, we saw that $32 \%$ (13/41) of the disease-causing genes in the MD patients were not included in the MitoCarta database for mitochondrial localized proteins at the time we identified the genetic defect (Table 3). Surprisingly, this included 5 genes with a literature reported function in mitochondrial metabolism (QRSL1, RRM2B, RRM1, c19orf12, TAZ). Furthermore, we identified 5 different genes with, so far, no reported function in mitochondrial metabolism (IER3IP1, SLC19A3 (2x), SLC16A2, IARS, CHRNE) to be involved in mitochondrial disease. Two additional genes that were lacking in the MitoCarta database were most likely not related to the mitochondrial symptoms of the patients, as these non-mitochondrial genes were, together with a primary mitochondrial gene defect, part of a complex multi-genic disease phenotype (ACY1, ANTXR2). 


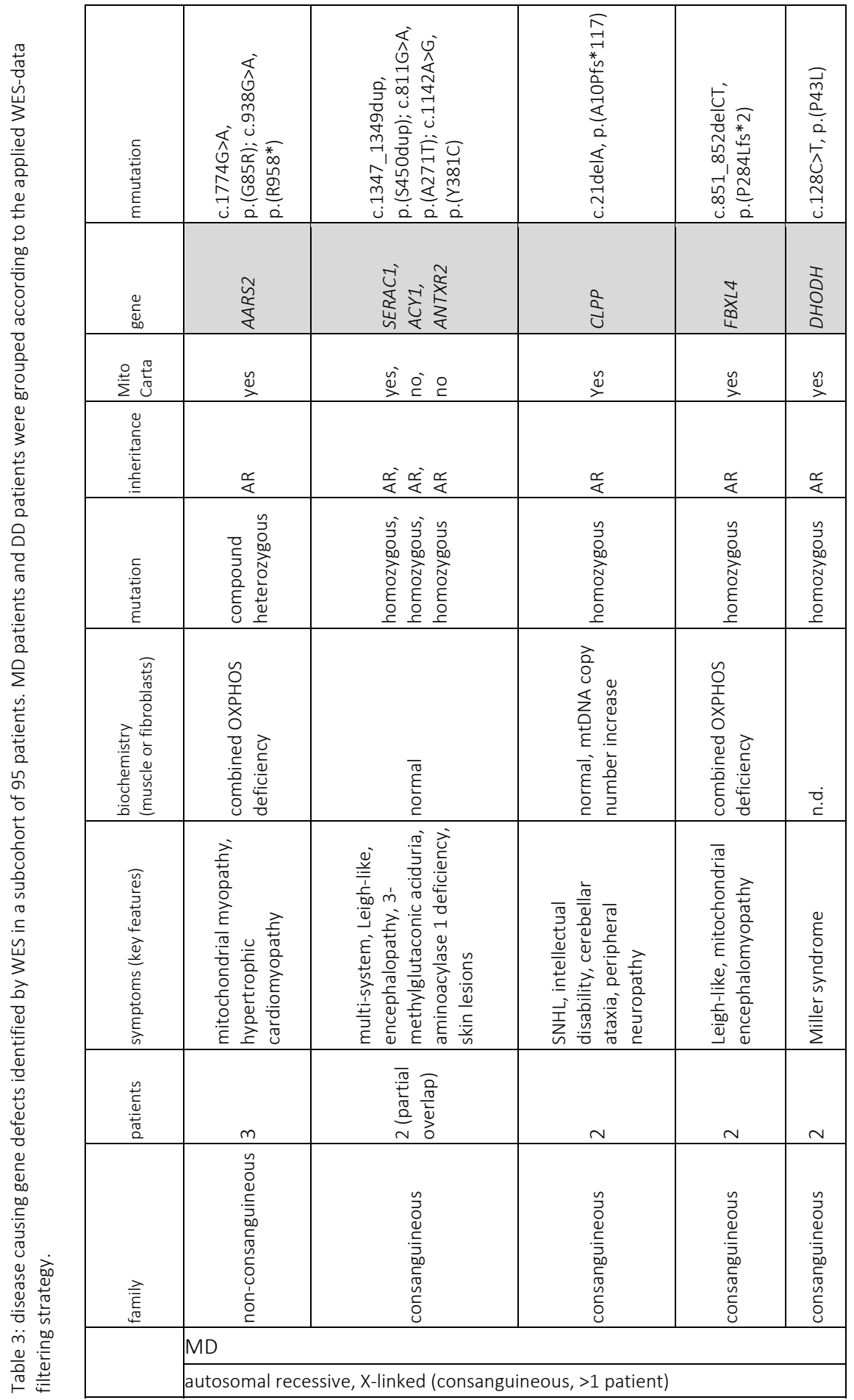




\begin{tabular}{|c|c|c|c|c|c|c|}
\hline 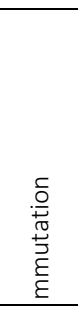 & 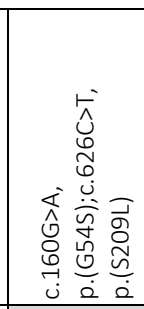 & 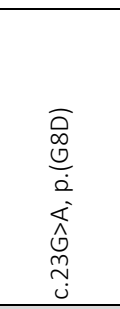 & 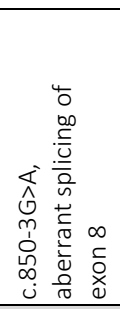 & 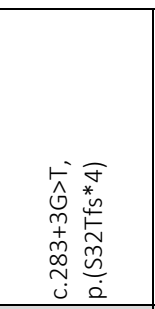 & 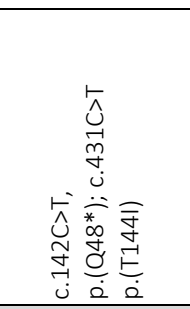 & 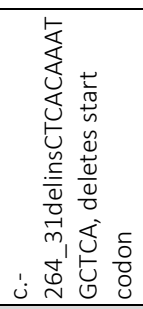 \\
\hline 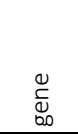 & $\underset{\substack{k \\
\Sigma}}{\Sigma}$ & $\begin{array}{l}\frac{1}{2} \\
\frac{1}{4} \\
\frac{1}{2} \\
2\end{array}$ & $\begin{array}{l}7 \\
\sqrt{1} \\
0 \\
0\end{array}$ & 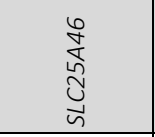 & $\sum_{\substack{\infty \\
\ll}}^{\infty}$ & ָ̃ \\
\hline 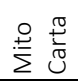 & $\stackrel{\varrho}{\rightleftharpoons}$ & $\stackrel{\Perp}{\Sigma}$ & ○ & $\stackrel{\varrho}{\beth}$ & $\stackrel{\circ}{\complement}$ & 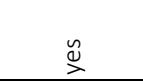 \\
\hline 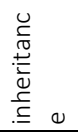 & $\frac{\alpha}{<}$ & 孚 & $\frac{\alpha}{4}$ & $\frac{\alpha}{4}$ & $\frac{⿱}{4}$ & $\frac{\Upsilon}{4}$ \\
\hline 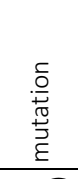 & 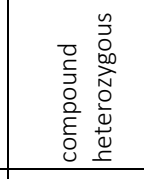 & 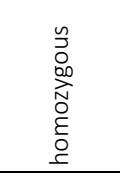 & 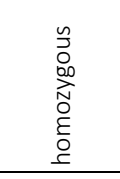 & 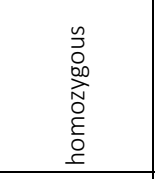 & 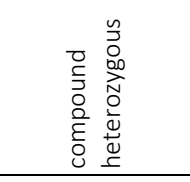 & 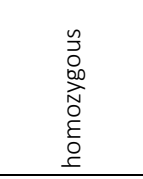 \\
\hline 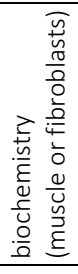 & 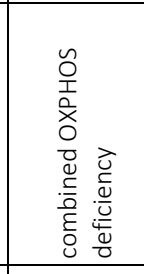 & 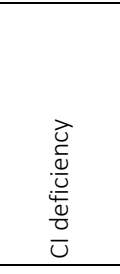 & 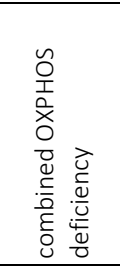 & 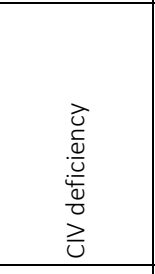 & 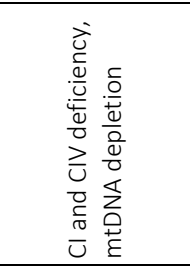 & 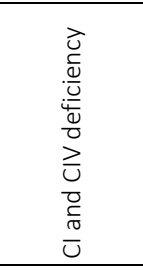 \\
\hline 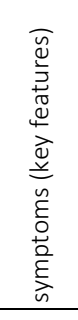 & 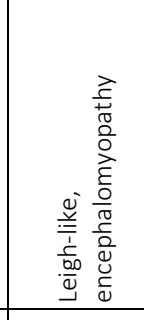 & 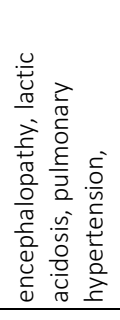 & 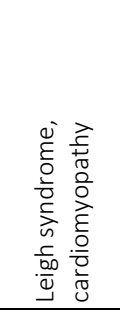 & 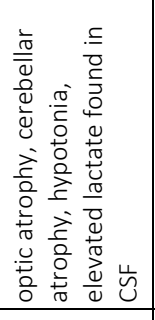 & 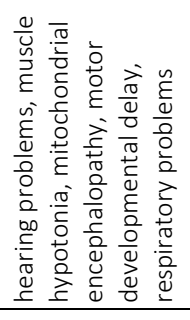 & 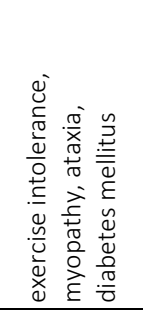 \\
\hline 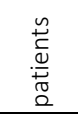 & $m$ & $r$ & $\sim$ & -1 & -1 & $r$ \\
\hline 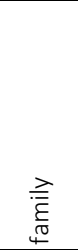 & 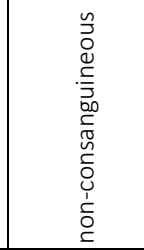 & 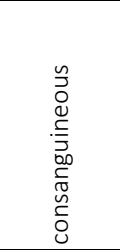 & 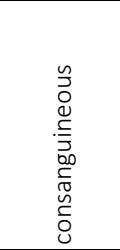 & 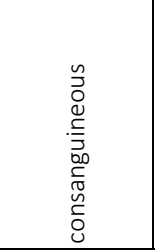 & 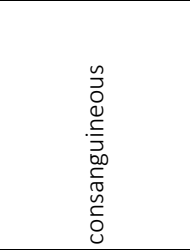 & 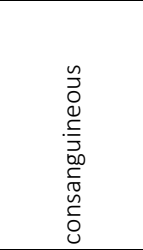 \\
\hline & \multicolumn{6}{|l|}{ MD } \\
\hline & autos & & & & & \\
\hline
\end{tabular}




\begin{tabular}{|c|c|c|c|c|c|c|}
\hline 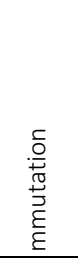 & 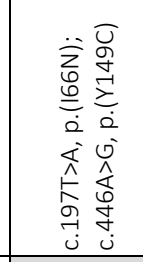 & 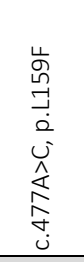 & 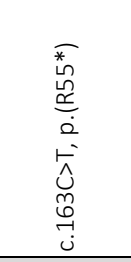 & 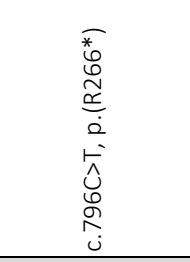 & 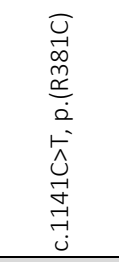 & 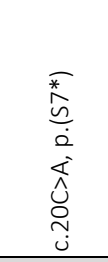 \\
\hline $\begin{array}{l}0 \\
\stackrel{0}{ \pm} \\
\text { D. } \\
\end{array}$ & ठิ & $\begin{array}{l}\frac{n}{4} \\
\frac{1}{3} \\
2 \\
2\end{array}$ & 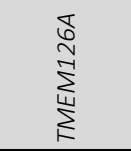 & $\begin{array}{l}\text { î } \\
\frac{0}{0} \\
\end{array}$ & $\sum_{\substack{\alpha \\
\alpha}}^{-1}$ & $\begin{array}{l}I \\
\text { I } \\
0 \\
0\end{array}$ \\
\hline 总 & $\stackrel{\varrho}{\rightleftharpoons}$ & $\stackrel{\dddot{\Perp}}{\rightleftharpoons}$ & $\stackrel{\varrho}{\rightleftharpoons}$ & $\stackrel{\varrho}{\rightleftharpoons}$ & $\stackrel{\circ}{\complement}$ & $\stackrel{\cong}{\beth}$ \\
\hline 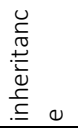 & $\frac{\Upsilon}{4}$ & $\frac{⿱}{4}$ & $\frac{⿱}{<}$ & 爰 & $\frac{9}{4}$ & $\stackrel{\frac{\alpha}{4}}{4}$ \\
\hline 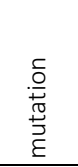 & 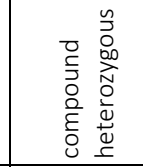 & 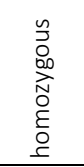 & 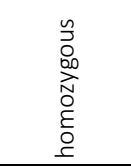 & 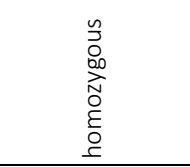 & 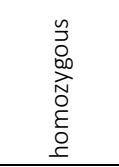 & 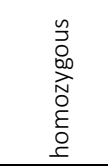 \\
\hline 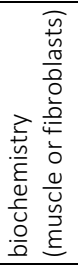 & 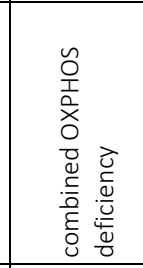 & 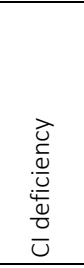 & $\begin{array}{l}\vec{u} \\
\frac{\bar{v}}{u} \\
\frac{u}{\underline{U}} \\
\frac{u}{0} \\
\bar{U}\end{array}$ & 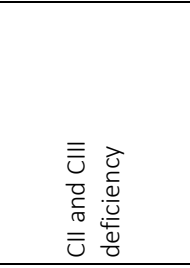 & 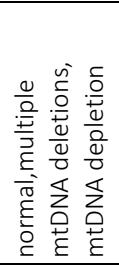 & $\underset{\check{c}}{\check{\Sigma}}$ \\
\hline 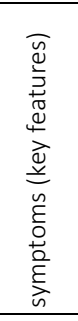 & 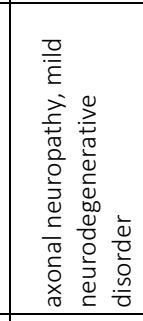 & 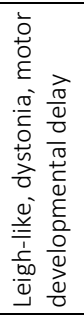 & 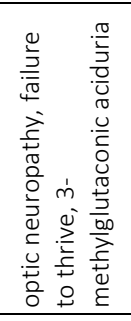 & 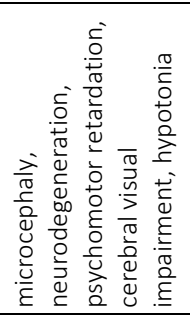 & 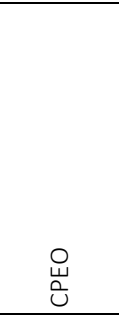 & 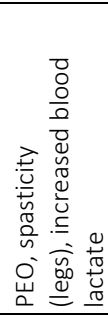 \\
\hline 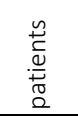 & $\sim$ & 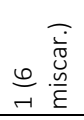 & 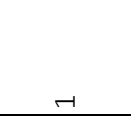 & $m$ & $\sim$ & $\rightarrow$ \\
\hline 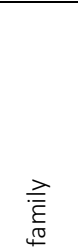 & 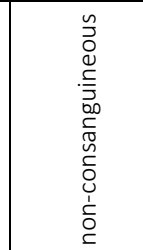 & 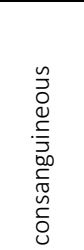 & 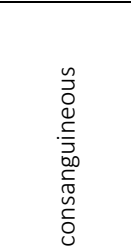 & 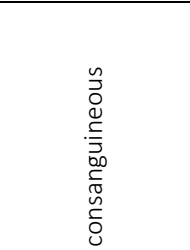 & 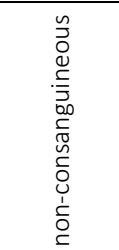 & 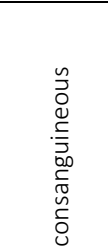 \\
\hline & \multicolumn{6}{|l|}{$M D$} \\
\hline & \multicolumn{6}{|c|}{ autosomal recessive, $X$-linked (consanguineous, $>1$ patient) } \\
\hline
\end{tabular}


Whole exome sequencing is the preferred, first strategy to identify known and novel causes of mitochondrial disease

\begin{tabular}{|c|c|c|c|c|c|c|}
\hline 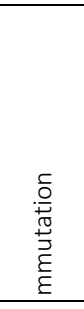 & 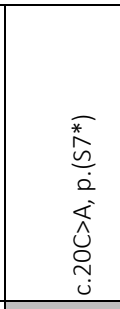 & 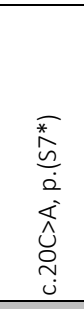 & 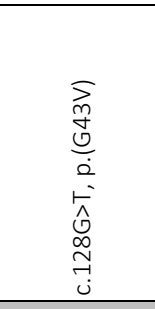 & 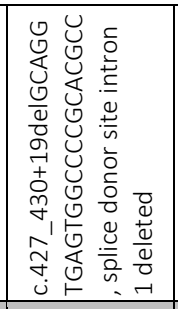 & 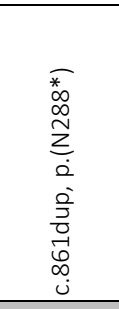 & 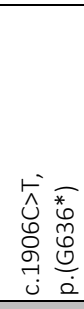 \\
\hline 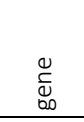 & 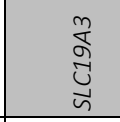 & 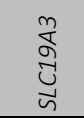 & $\begin{array}{l}\frac{a}{m} \\
\underline{m} \\
\underline{\omega}\end{array}$ & 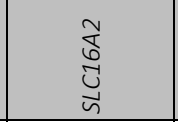 & $\begin{array}{l}\hat{0} \\
\text { जे }\end{array}$ & $\begin{array}{l}\sum_{0}^{n} \\
\vdots \\
j\end{array}$ \\
\hline 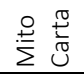 & $\stackrel{\circ}{\complement}$ & $\stackrel{\circ}{\complement}$ & $\stackrel{\circ}{\complement}$ & $\stackrel{\circ}{\complement}$ & 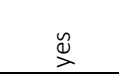 & $\stackrel{\circ}{\complement}$ \\
\hline 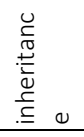 & 妥 & $\frac{⿱}{4}$ & $\frac{\alpha}{4}$ & $\frac{⿱}{<}$ & 产 & $\frac{⿱}{<}$ \\
\hline 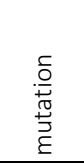 & 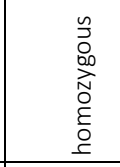 & 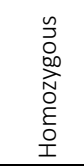 & 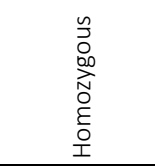 & 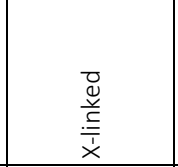 & 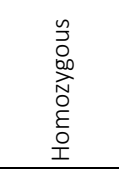 & 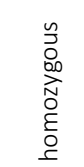 \\
\hline 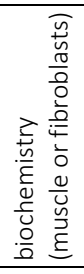 & 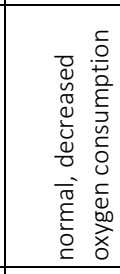 & 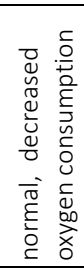 & 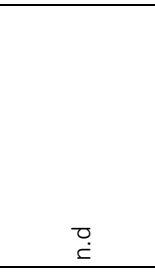 & 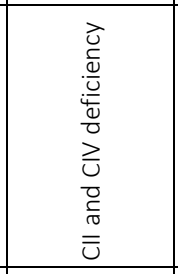 & $\begin{array}{l}\overline{\widetilde{\sigma}} \\
\xi \\
\bar{\Xi}\end{array}$ & வ் \\
\hline 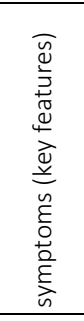 & 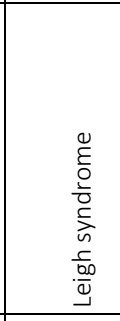 & 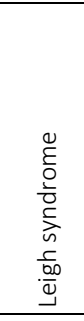 & 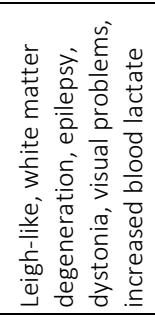 & 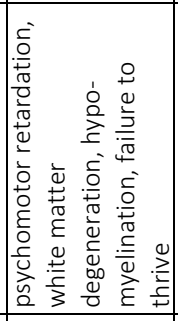 & 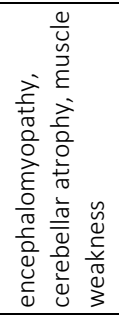 & 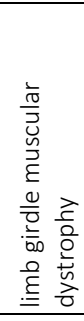 \\
\hline 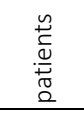 & $m$ & $\sim$ & $\rightarrow$ & $\sim$ & $\sim$ & $\neg$ \\
\hline 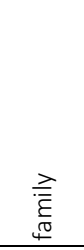 & 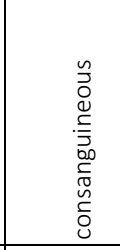 & 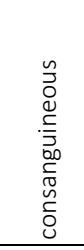 & 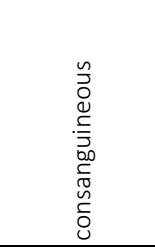 & 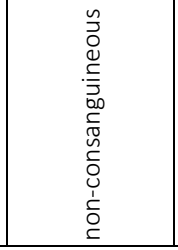 & 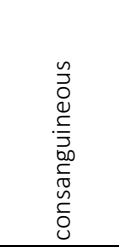 & 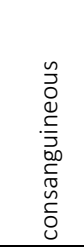 \\
\hline & \multicolumn{6}{|l|}{ MD } \\
\hline & \multicolumn{6}{|c|}{ autosomal recessive, $\mathrm{X}$-linked (consanguineous, $>1$ patient) } \\
\hline
\end{tabular}




\begin{tabular}{|c|c|c|c|c|c|c|c|}
\hline 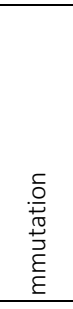 & 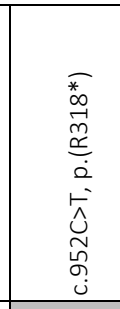 & 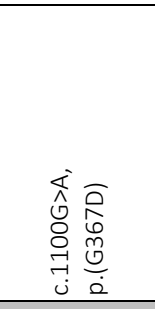 & 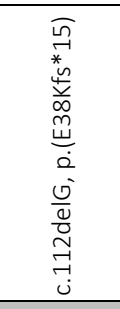 & 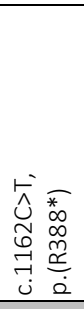 & 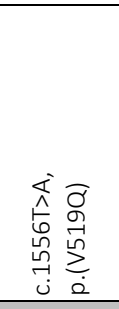 & 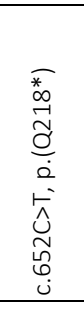 & 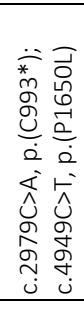 \\
\hline $\begin{array}{l}\stackrel{0}{\tilde{\nu}} \\
\stackrel{\Sigma}{\infty} \\
\end{array}$ & $\begin{array}{l}\sum_{+}^{-1} \\
\frac{+}{4}\end{array}$ & \begin{tabular}{l}
$m$ \\
\multirow{2}{*}{}
\end{tabular} & $\begin{array}{l}m \\
\stackrel{0}{0} \\
\vdots\end{array}$ & $\underset{\vdots}{\vdots}$ & $\frac{\tilde{c}}{z}$ & 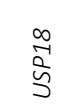 & 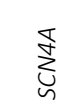 \\
\hline 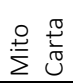 & 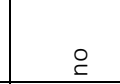 & $\stackrel{\circ}{\complement}$ & 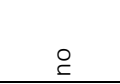 & 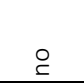 & 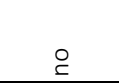 & 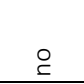 & 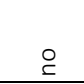 \\
\hline 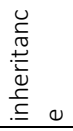 & $\frac{\alpha}{<}$ & $\frac{\Upsilon}{4}$ & $\frac{⿱}{4}$ & $\stackrel{\frac{c}{4}}{4}$ & $\stackrel{\frac{\alpha}{4}}{4}$ & $\frac{⿱}{4}$ & $\frac{9}{4}$ \\
\hline 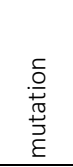 & 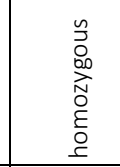 & 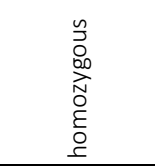 & 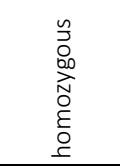 & 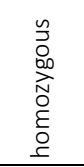 & 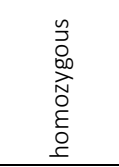 & 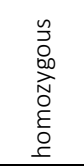 & 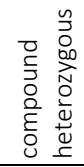 \\
\hline 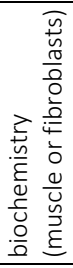 & $\begin{array}{l}\overline{\widetilde{\pi}} \\
\underline{\xi} \\
\stackrel{0}{\complement}\end{array}$ & 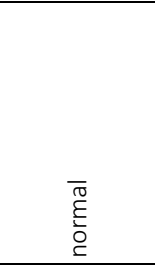 & 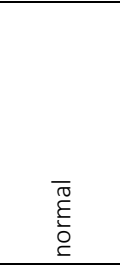 & $\begin{array}{l}\overline{\widetilde{\pi}} \\
\underline{\xi} \\
\stackrel{0}{\complement}\end{array}$ & 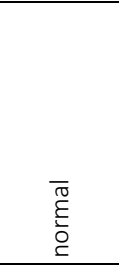 & $\begin{array}{l}\bar{\pi} \\
\underline{\xi} \\
\varnothing\end{array}$ & 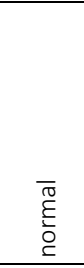 \\
\hline 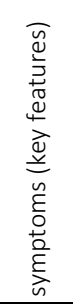 & 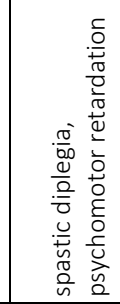 & 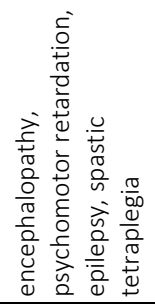 & 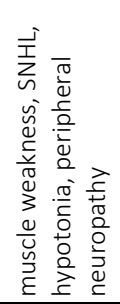 & 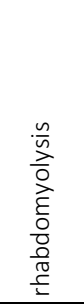 & 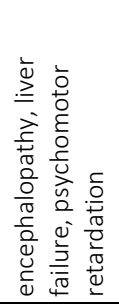 & 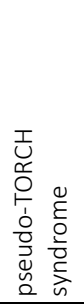 & 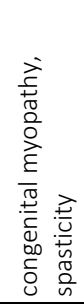 \\
\hline 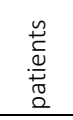 & $r$ & $r$ & 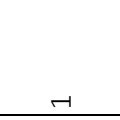 & $\sim$ & $\sim$ & $\sim$ & $\sim$ \\
\hline 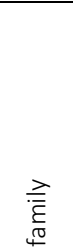 & 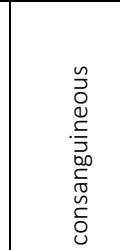 & 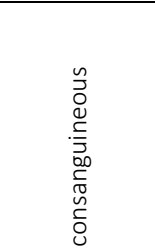 & 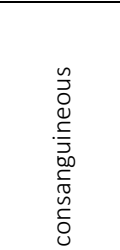 & 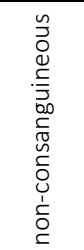 & 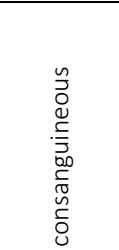 & 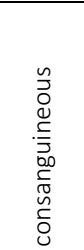 & 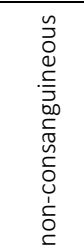 \\
\hline & \multicolumn{7}{|l|}{$M D$} \\
\hline & \multicolumn{7}{|c|}{ autosomal recessive, $X$-linked (consanguineous, $>1$ patient) } \\
\hline
\end{tabular}




\begin{tabular}{|c|c|c|c|c|c|c|}
\hline 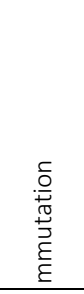 & 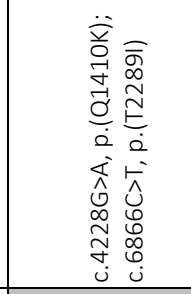 & 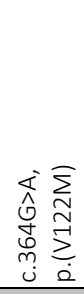 & 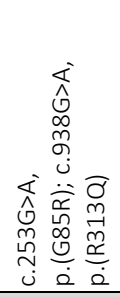 & 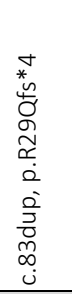 & 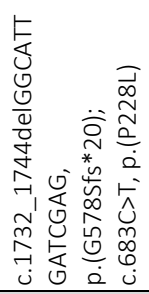 & 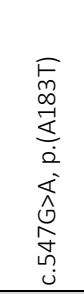 \\
\hline 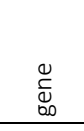 & 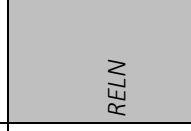 & $\begin{array}{l}\hat{u} \\
\vdots \\
\Sigma \\
z\end{array}$ & $\frac{\stackrel{0}{E}}{\Sigma}$ & $\begin{array}{l}\text { İ } \\
\frac{1}{3} \\
2 \\
2\end{array}$ & $\frac{n}{\frac{2}{2}}$ & $\begin{array}{l}2 \\
\frac{1}{2} \\
\stackrel{2}{2} \\
\end{array}$ \\
\hline$\stackrel{\circ}{\stackrel{0}{\stackrel{5}{*}}}$ & $\stackrel{\circ}{\complement}$ & $\stackrel{\Perp}{\rightleftharpoons}$ & $\stackrel{\varrho}{\rightleftharpoons}$ & $\stackrel{\check{\varpi}}{>}$ & $\stackrel{\check{\Perp}}{\beth}$ & $\stackrel{\varrho}{\supset}$ \\
\hline 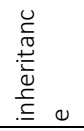 & $\stackrel{\frac{\alpha}{4}}{4}$ & $\frac{⿱}{4}$ & $\frac{⿱}{4}$ & $\stackrel{\Upsilon}{<}$ & $\frac{a}{4}$ & $\frac{a}{4}$ \\
\hline 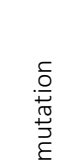 & 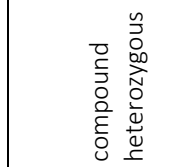 & 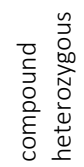 & 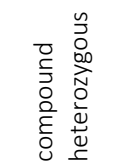 & 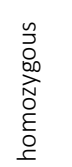 & 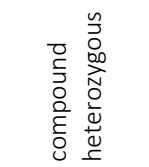 & 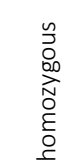 \\
\hline 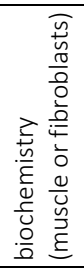 & & 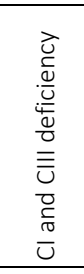 & 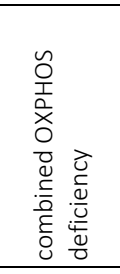 & 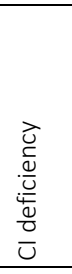 & 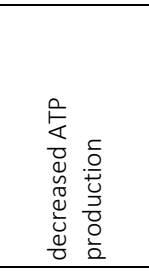 & 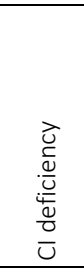 \\
\hline 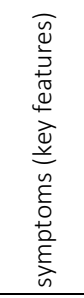 & 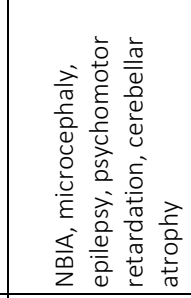 & 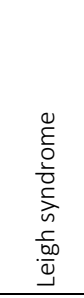 & 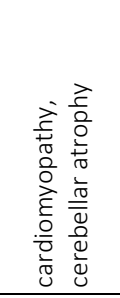 & 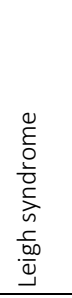 & 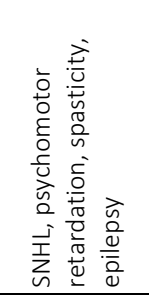 & 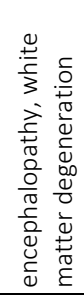 \\
\hline 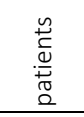 & $r$ & $r$ & -1 & 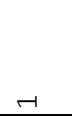 & -1 & $-r$ \\
\hline 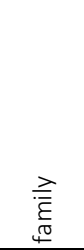 & 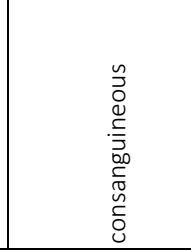 & 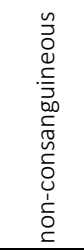 & 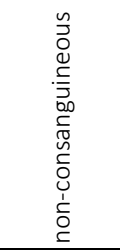 & 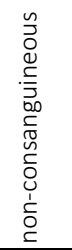 & 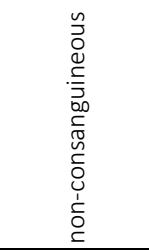 & 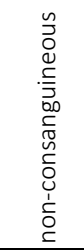 \\
\hline & \multicolumn{6}{|l|}{$M D$} \\
\hline & \multicolumn{6}{|c|}{ autosomal recessive, $\mathrm{X}$-linked (consanguineous, $>1$ patient) } \\
\hline
\end{tabular}




\begin{tabular}{|c|c|c|c|c|c|c|}
\hline 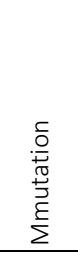 & 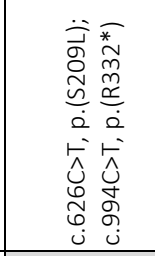 & 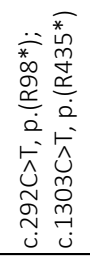 & 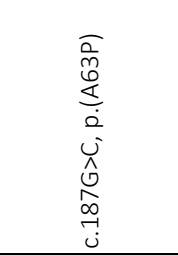 & 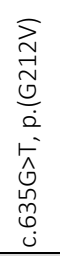 & 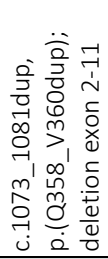 & 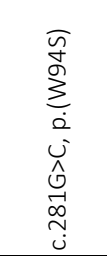 \\
\hline 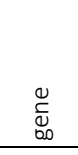 & 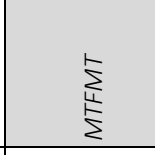 & $\begin{array}{l}\vec{x} \\
\mathbb{x} \\
\end{array}$ & $\begin{array}{l}\text { के } \\
\text { ธे } \\
\text { ปे }\end{array}$ & 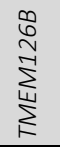 & $\sum_{\substack{K \\
\Sigma}}^{2}$ & $\begin{array}{l}\frac{N}{4} \\
\frac{1}{k} \\
\end{array}$ \\
\hline 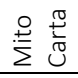 & $\stackrel{\varrho}{\rightleftharpoons}$ & $\stackrel{\varrho}{\rightleftharpoons}$ & $\stackrel{\circ}{\complement}$ & $\stackrel{\varrho}{\rightleftharpoons}$ & $\stackrel{\varrho}{\rightleftharpoons}$ & $\stackrel{\varrho}{\rightleftharpoons}$ \\
\hline 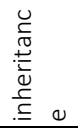 & $\frac{9}{4}$ & $\stackrel{\frac{c}{4}}{4}$ & $\frac{⿱}{4}$ & $\stackrel{\Upsilon}{<}$ & $\frac{⿱}{4}$ & $\frac{a}{4}$ \\
\hline 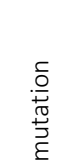 & 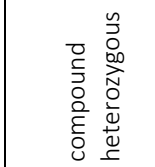 & 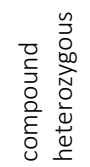 & 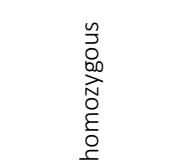 & 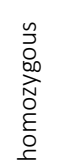 & 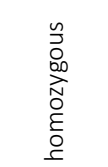 & 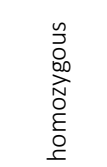 \\
\hline 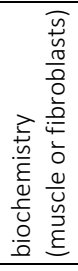 & 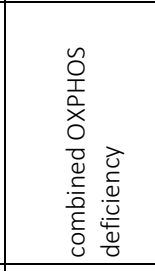 & $\underset{\check{c}}{\check{c}}$ & 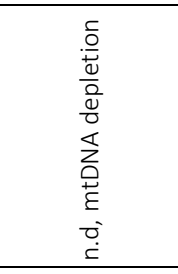 & 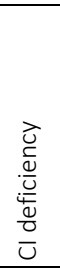 & 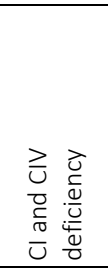 & 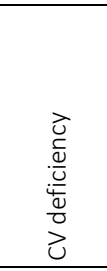 \\
\hline 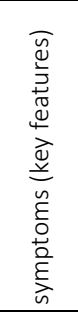 & 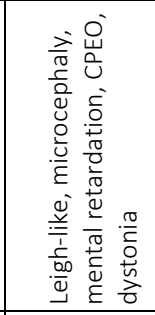 & 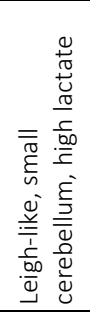 & 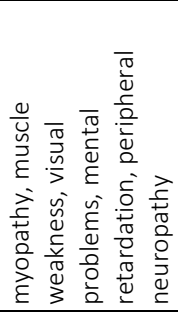 & 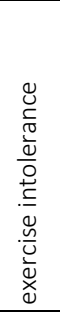 & 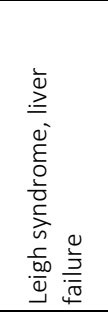 & 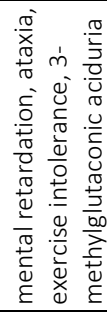 \\
\hline 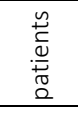 & - & -1 & - & $r$ & - & 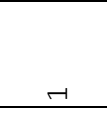 \\
\hline 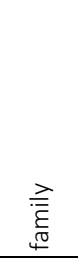 & 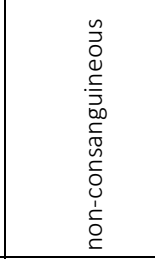 & 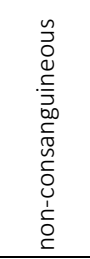 & 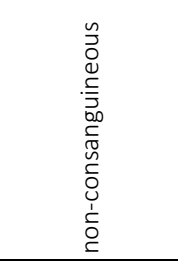 & 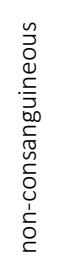 & 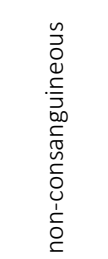 & 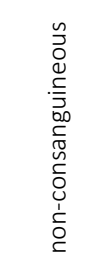 \\
\hline & \multicolumn{6}{|l|}{$M D$} \\
\hline & \multicolumn{6}{|c|}{ autosomal recessive, $X$-linked (consanguineous, $>1$ patient) } \\
\hline
\end{tabular}


Whole exome sequencing is the preferred, first strategy to identify known and novel causes of mitochondrial disease

\begin{tabular}{|c|c|c|c|c|c|c|}
\hline 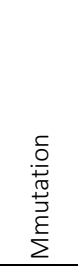 & 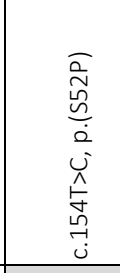 & 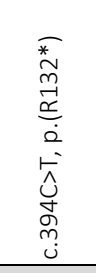 & 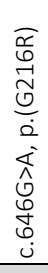 & 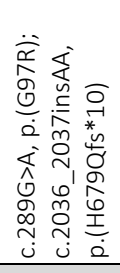 & 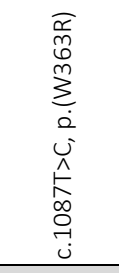 & 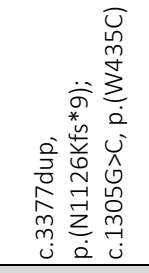 \\
\hline 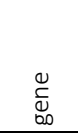 & 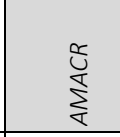 & 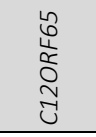 & E & 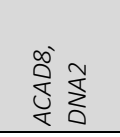 & 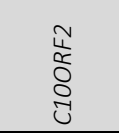 & $\begin{array}{l}\text { L̃ } \\
\underline{5}\end{array}$ \\
\hline 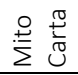 & $\stackrel{\varrho}{\rightleftharpoons}$ & $\stackrel{\varrho}{\rightleftharpoons}$ & $\cong$ & 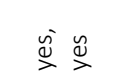 & $\stackrel{\varrho}{\supset}$ & 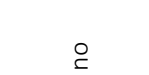 \\
\hline 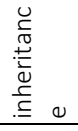 & $\frac{⿱}{<}$ & 孚 & 产 & 产是 & 安 & 妥 \\
\hline 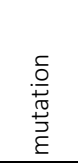 & 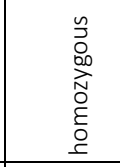 & 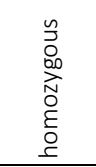 & 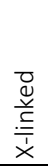 & 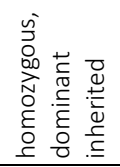 & $\begin{array}{l}\text { \# } \\
\sum_{0} \\
0 \\
\frac{5}{5} \\
5 \\
\end{array}$ & 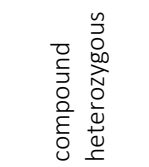 \\
\hline 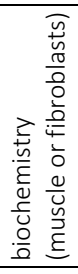 & ذْ & 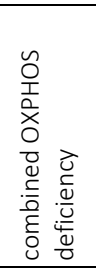 & வ் & ذ্் & 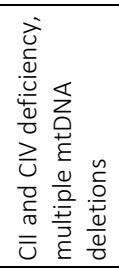 & 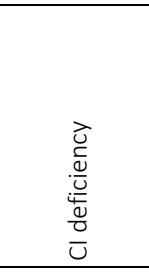 \\
\hline 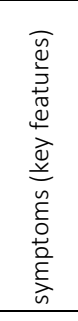 & 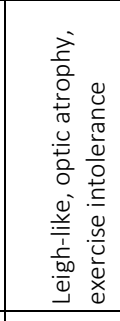 & 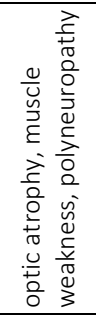 & 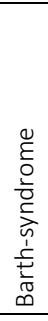 & 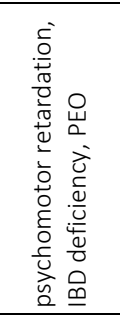 & 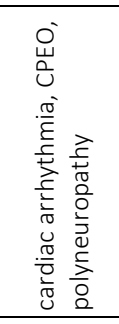 & 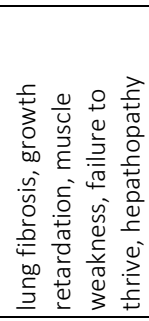 \\
\hline 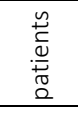 & -1 & -1 & -1 & $-r$ & $-r$ & -1 \\
\hline 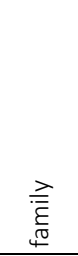 & 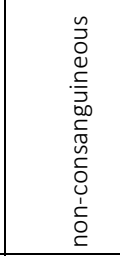 & 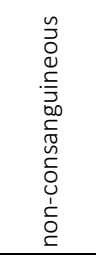 & 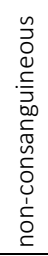 & 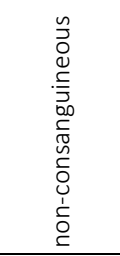 & 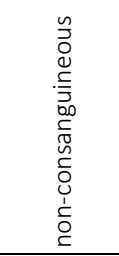 & 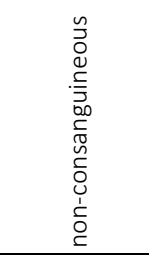 \\
\hline & \multicolumn{6}{|l|}{ MD } \\
\hline & \multicolumn{6}{|c|}{ autosomal recessive, $X$-linked (consanguineous, $>1$ patient) } \\
\hline
\end{tabular}




\begin{tabular}{|c|c|c|c|c|c|c|}
\hline 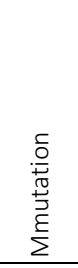 & 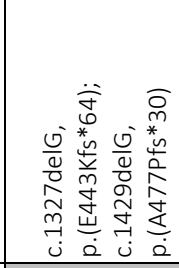 & 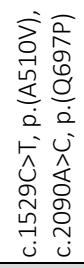 & 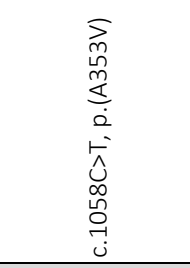 & 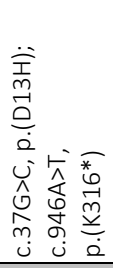 & 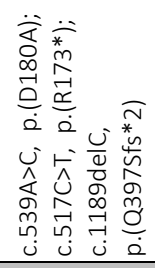 & $\begin{array}{l}\widehat{y} \\
0 \\
0 \\
0 \\
\dot{0} \\
i \\
0 \\
0 \\
0 \\
ن \\
ن\end{array}$ \\
\hline $\begin{array}{l}0 \\
\stackrel{్}{ \pm} \\
\stackrel{0}{0} \\
\end{array}$ & $\begin{array}{l}\text { 崖 } \\
\frac{⿱ 乛}{ \pm} \\
\end{array}$ & $\begin{array}{l}\text { जे } \\
\text { जे }\end{array}$ & $\sum_{\sum}^{N}$ & 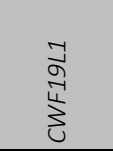 & 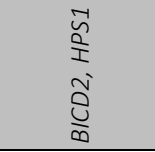 & $\underset{E}{\mathbb{E}}$ \\
\hline 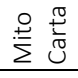 & 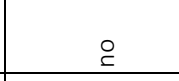 & $\stackrel{\varrho}{\rightleftharpoons}$ & $\stackrel{\varrho}{\rightleftharpoons}$ & $\stackrel{\circ}{\complement}$ & ¿̇ & $\stackrel{\circ}{\complement}$ \\
\hline 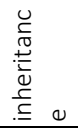 & 孚 & $\frac{\alpha}{4}$ & 安 & $\frac{⿱}{<}$ & $\begin{array}{l}\frac{r}{4} \\
0 \\
<\end{array}$ & 是 \\
\hline 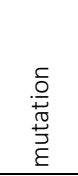 & 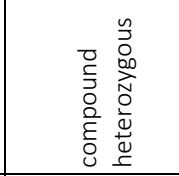 & 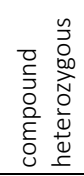 & $\begin{array}{l}0 \\
0 \\
c \\
\frac{0}{0}\end{array}$ & 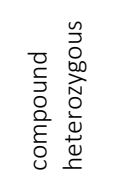 & 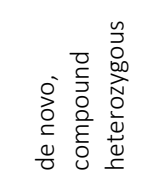 & $\begin{array}{l}0 \\
\text { o } \\
\frac{1}{0} \\
\frac{0}{0}\end{array}$ \\
\hline 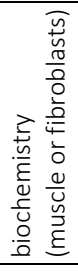 & 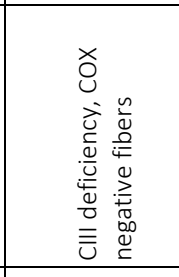 & $\begin{array}{l}\overline{\widetilde{O}} \\
\underline{\xi} \\
\mathrm{C}\end{array}$ & வ் & 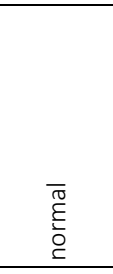 & 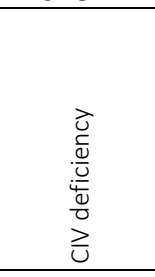 & $\dot{\ddot{c}}$ \\
\hline 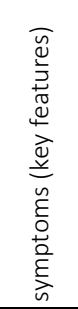 & 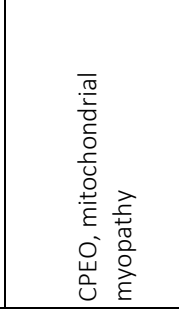 & 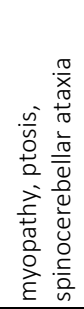 & 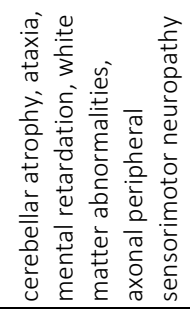 & 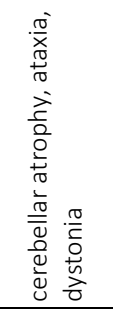 & 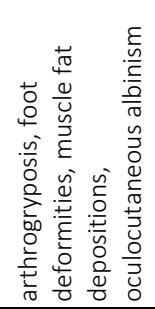 & 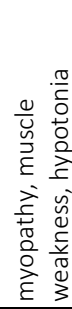 \\
\hline 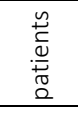 & 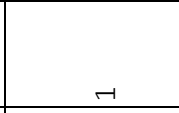 & $\neg$ & 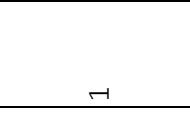 & 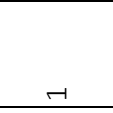 & - & 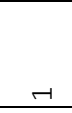 \\
\hline 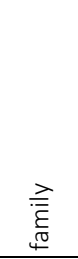 & 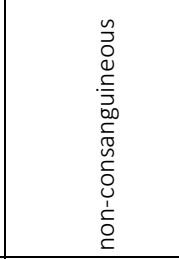 & 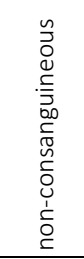 & 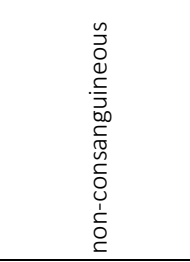 & 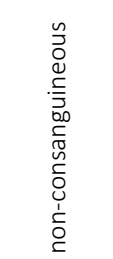 & 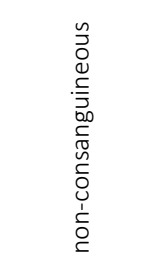 & 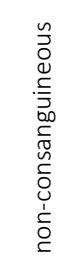 \\
\hline & \multicolumn{6}{|l|}{$M D$} \\
\hline & \multicolumn{6}{|c|}{ autosomal recessive, $\mathrm{X}$-linked (consanguineous, $>1$ patient) } \\
\hline
\end{tabular}




\begin{tabular}{|c|c|c|c|c|c|}
\hline 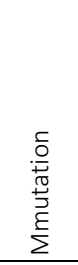 & 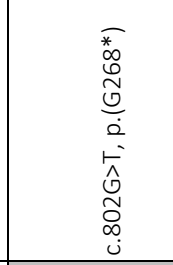 & 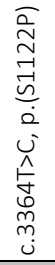 & 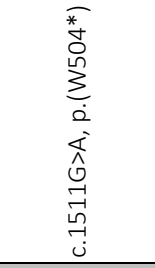 & 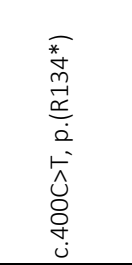 & 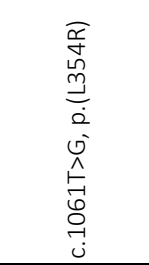 \\
\hline 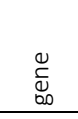 & 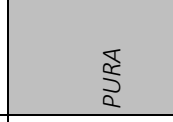 & 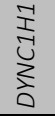 & $\sum_{\leq}^{\infty}$ & $\begin{array}{l}\text { n } \\
\text { st } \\
3 \\
3\end{array}$ & ভ্য \\
\hline 올 & $\stackrel{\circ}{\complement}$ & $\stackrel{1}{\complement}$ & $\stackrel{\circ}{\complement}$ & $\stackrel{\circ}{\complement}$ & $\stackrel{9}{\complement}$ \\
\hline 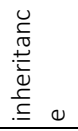 & 是 & 是 & 是 & 口 & ○ \\
\hline 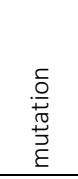 & $\begin{array}{l}0 \\
0 \\
c \\
0 \\
0 \\
\end{array}$ & $\begin{array}{l}0 \\
0 \\
c \\
\frac{0}{0} \\
\end{array}$ & $\begin{array}{l}0 \\
0 \\
c \\
\text { d } \\
\end{array}$ & 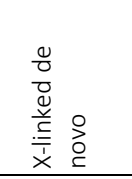 & 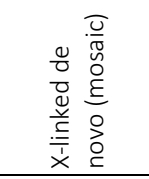 \\
\hline 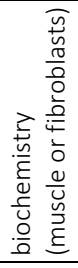 & 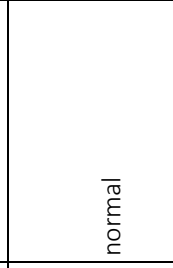 & $\begin{array}{l}\overline{\widetilde{\sigma}} \\
\underline{\Xi} \\
\check{\varrho}\end{array}$ & $\begin{array}{l}\bar{\pi} \\
\underline{\xi} \\
\check{\complement}\end{array}$ & $\begin{array}{l}\overline{\widetilde{\pi}} \\
\underline{\xi} \\
\check{\complement}\end{array}$ & $\begin{array}{l}\bar{\pi} \\
\underline{\xi} \\
\bar{\complement}\end{array}$ \\
\hline 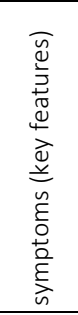 & 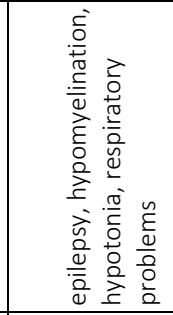 & 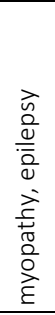 & 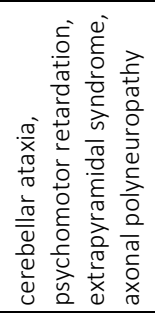 & 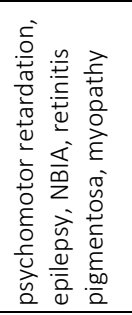 & 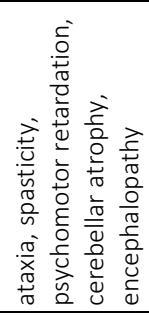 \\
\hline 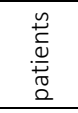 & $r$ & 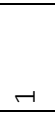 & $\rightarrow$ & $r$ & -1 \\
\hline 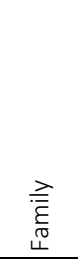 & 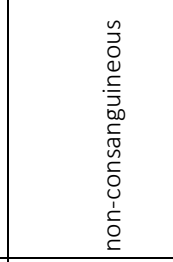 & 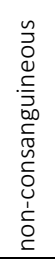 & 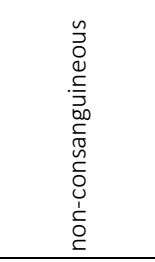 & 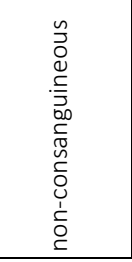 & 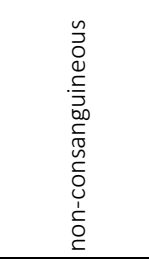 \\
\hline & \multicolumn{5}{|l|}{ MD } \\
\hline & \multicolumn{5}{|c|}{ autosomal recessive, $\mathrm{X}$-linked (consanguineous, $>1$ patient) } \\
\hline
\end{tabular}

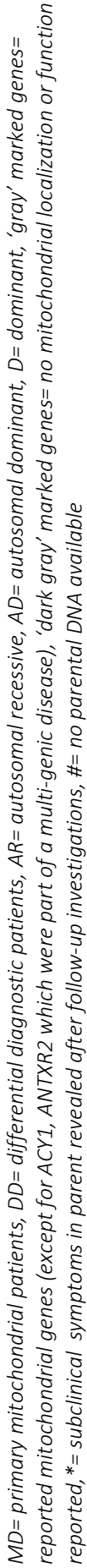


Genes identified in MD patients were clustered according to their function (Table 4). Most genetic defects were detected in genes related to mitochondrial protein metabolism (protein translation and degradation) and OXPHOS function (ETC subunits, assembly factors and cofactors). The first group mainly consisted of mitochondrial tRNA synthethases, transferases and modification enzymes, where the resulting defects in mitochondrial protein synthesis (AARS2, MTFMT, MTO1, QRSL1, TRMU, C12ORF65) especially manifested with combined OXPHOS deficiencies (not measured for KARS). Among the group of OXPHOS-associated genes, defects in complex I subunits and assembly factors (NDUFA12, NDUFS7, NDUFV2, TMEM126A, TMEM126B, NDUFAF4, NDUFAF5) were most prevalent, all resulting in a significant complex I deficiency at the biochemical level. NDUFS7 resulted in an additional complex III deficiency. Defects in NDUFS7, NDUFA12 and NDUFAF5 caused Leigh-syndrome or a Leigh-like-phenotype. The third largest group represented genes involved in mtDNA metabolism, required for mtDNA maintenance, replication and nucleotide metabolism. Interacting gene $R R M 2 B$ and $R R M 1$ both resulted in mtDNA depletion, where the RRM1 defect additionally caused multiple mtDNA deletions. On the other hand, mutations in the RMM2B interacting protein PYCR2 did not reveal any mtDNA abnormalities. Furthermore, mutations in c10orf2 (twinkle) and DNA2 displayed multiple mtDNA deletions in muscle. Interestingly, we found the mitochondrial localized, but functionally uncharacterized, c19orf12 gene to be associated with mtDNA depletion. Among the MD patients, we identified mutations in 5 genes without a reported mitochondrial function or localization, which most likely underlie the OXPHOS deficiency and/or classical mitochondrial disease symptoms in the patient. This included the functionally uncharacterized IER3IP1 gene and 4 genes functional in different cellular processes, including the transporter proteins SLC19A3 (thiamine) and SLC16A2 (thyroid hormones), tRNA-synthetase IARS, and acetylcholine receptor subunit CHRNE. As indicated in Table 5, the DD patient group included only 2 mitochondrial gene defects, SPG7 as part of hereditary spastic paraplegia's and MFN2 underlying a Charcot-Marie-Tooth disease phenotype. 
Table 4: Disease causing nuclear genes, identified in patients with a primary mitochondrial disorder (MD patients), clustered according to their function in mitochondrial metabolism.

\begin{tabular}{|c|c|c|}
\hline gene & function & reference \\
\hline \multicolumn{3}{|c|}{ mitochondrial substrate import } \\
\hline$S L C 25 A 32$ & mitochondrial folate transporter & Haitina et al., 2006 \\
\hline \multicolumn{3}{|c|}{ mitochondrial substrate metabolism } \\
\hline ACAD8 & Isobutyryl-CoA dehydrogenase & Nguyen et al., 2002 \\
\hline$A M A C R$ & Alpha-methylacyl-CoA racemase & Mubiru et al., 2004 \\
\hline$O G D H$ & alpha-ketoglutarate dehydrogenase & Szabo et al., 1994 \\
\hline \multicolumn{3}{|c|}{ mitochondrial protein metabolism; translation and degradation } \\
\hline AARS2 & alanyl-tRNA synthetase & Gotz et al., 2011 \\
\hline MTFMT $(2 x)$ & mitochondrial methionyl-tRNA formyltransferase & Takeuchi et al., 1998 \\
\hline MTO1 & tRNA modification and protein synthesis & Ghezzi et al., 2012 \\
\hline KARS & lysyl-tRNA synthetase & Targoff et al., 1993 \\
\hline QRSL1 & glu-tRNA synthetase & Nagao et al., 2009 \\
\hline TRMU & mitochondrial tRNA modification & Yan et al., 2005 \\
\hline C12ORF65 & release proteins from ribosomes & Antonicka et al., 2010 \\
\hline$C L P P$ & mitochondrial proteolytic complex & Jenkinson et al., 2013 \\
\hline \multicolumn{3}{|c|}{ mitochondrial phospholipid metabolism } \\
\hline SERAC1 & phosphatidylglycerol remodeling & Wortmann et al., 2012 \\
\hline$T A Z$ & cardiolipin remodeling & Acehan et al., 2011 \\
\hline \multicolumn{3}{|c|}{ ETC subunits, assembly factors, cofactors } \\
\hline NDUFA12 & complex I subunit & Triepels et al., 2000 \\
\hline NDUFS7 & complex I subunit & Visch et al., 2004 \\
\hline NDUFV2 & complex I subunit & de Coo et al., 1995 \\
\hline COQ7 & coenzyme $Q$ biosynthesis & Freyer et al., 2015 \\
\hline TMEM126B & complex I assembly factor & Andrews et al., 2013 \\
\hline ATPAF2 & complex $V$ assembly factor & Wang et al. (2001) \\
\hline NDUFAF5 & complex I assembly factor & Sugiana et al., 2008 \\
\hline NDUFAF4 & complex I assembly factor & Karp et al., 2004 \\
\hline TMEM126A & complex I assembly factor & Wessels et al., 2013 \\
\hline \multicolumn{3}{|c|}{ mtDNA maintanance, replication and nucleotide metabolism } \\
\hline$R R M 2 B$ & ribonucleotide reductase & Bourdon et al., 2007 \\
\hline$F B X L 4(2 x)$ & phosphorylation-dependent ubiquitination & Bonnen et al., 2013 \\
\hline DNA2 & mtDNA replication, mtDNA base-excision repair & Ronchi et al., 2013 \\
\hline PYCR2 & Pyrroline-5-carboxylate reductase & Mei-Ling Kuo et al., 2016 \\
\hline$R R M 1$ & ribonucleotide reductase & Pavloff et al., 1992 \\
\hline DHODH & pyrimidine biosynthesis & JingXian Fang et al., 2013 \\
\hline C10ORF2 & mtDNA helicase & Spelbrink et al., 2001 \\
\hline \multicolumn{3}{|c|}{ mitochondrial fusion and fision } \\
\hline SLC25A46 & interacts with mitochondrial fusion machinery & Janer et al., 2016 \\
\hline \multicolumn{3}{|c|}{ mitochondrial localization } \\
\hline C19orf12 & function unclear & Landoure et al., 2013 \\
\hline
\end{tabular}


no mitochondrial localization or function reported

\begin{tabular}{lll} 
SLC19A3 $(2 x)$ & transmembrane thiamine transporter & Vernau et al., 2015 \\
IER3IP1 & function unclear & Yiu et al., 2004 \\
SLC16A2 & transporter of thyroid hormones & Wrutniak-Cabello et al., 2001 \\
CHRNE & acetylcholine receptor subunit & Witzemann et al., 1996 \\
IARS & Isoleucyl-tRNA synthetase & Kopajtich et al., 2016 \\
\hline
\end{tabular}

*ACY1 and ANTXR2 were excluded

As indicated in Table 5, the DD patient group included only 2 mitochondrial gene defects, SPG7 as part of hereditary spastic paraplegia's and MFN2 underlying a CharcotMarie-Tooth disease phenotype.

Table 5: disease causing nuclear genes, identified in DD patients, classified according to their function in mitochondrial metabolism.

\begin{tabular}{ll}
\hline gene $\quad$ function & Reference \\
\hline $\begin{array}{l}\text { mitochondrial protein metabolism; degradation } \\
\text { SPG7 part of the m-AAA metalloproteinase complex }\end{array}$ & Warnecke et al, 2007 \\
mitochondrial fusion and fision & \\
MFN2 $\quad$ mitochondrial fusion & Santel et al., 2001 \\
\hline
\end{tabular}

\section{DISCUSSION}

We used a next-generation sequencing strategy in a cohort of 85 patients with primary mitochondrial disease and 33 patients, where a mitochondrial cause is part of the differential diagnosis. In $69 \%$ of the patients we identified a disease causing genetic defect, of which $20 \%$ was solved by mtDNA sequencing and $49 \%$ by subsequent WES analysis. Compared to conventional Sanger sequencing methods, which only solved $11 \%[25]$, primarily due to limitations on the number of genes being sequenced, this is a major step forward. Selective sequencing of targeted gene panels in two studies, including 1598 and 1034 mitochondrial disease genes, resulted in respectively 22\% (102 suspected mitochondrial patients)[26] and 24\% (42 mitochondrial patients)[27] diagnostic yield. This is also much less than in our study, which can be explained by the incompleteness of the panels (more details below). In a previous next-generation sequencingbased study, which included 113 paediatric patients with suspected mitochondrial disease, screening both the mtDNA and the exome, resulted in a diagnostic yield of 59\%[28]. Another study performing WES in 109 paediatric and young adult patients with suspected mitochondrial disease, established a genetic diagnosis in $39 \%$ of the patient cohort (after exclusion of mtDNA mutations)[21]. Our paediatric patient group consisted of 92 patients, for which we could establish a genetic diagnosis in 67\% (10 mtDNA and 52 nuclear gene mutations). Our higher yield is most likely caused by a complete analysis of both the mtDNA and the exome, a strong selection for genetic 
cases and the follow-up investigations in case of an unknown gene. In conclusion, our data shows that mtDNA sequencing followed by a complete whole exome analysis is the preferred strategy to identify the genetic basis of heterogeneous mitochondrial disease.

As the mtDNA genome is relatively small, NGS allows cost effective and efficient testing of many patient samples within a single run, with sufficient sensitivity on blood DNA. Where traditionally several complementary methods had to be used for detection and quantification of mtDNA mutations (e.g. RFLP, ARMS-qPCR, sanger sequencing, Mito-CHIP, Southern blot analysis), NGS can be used as a single method for identifying point mutations and indels, large deletion breakpoints and quantifying heteroplasmy levels of point mutations and small indels with high sensitivity and specificity[14]. Only to accurately measure heteroplasmy levels of large mtDNA deletions and mtDNA depletion, additional qPCR based quantification is required. NGS of the mtDNA solved $20 \%$ of our patient cohort (all MD patients), mainly, but not only, consisting of patients with early disease onset (77\% paediatric patients). In the pure paediatric subgroup, primary mtDNA mutations accounted for $11 \%(10 / 92)$ of the genetic defects, which is in line with earlier reports that estimated involvement of a mtDNA defect in $<20 \%$ of the paediatric mitochondrial patients[29]. In our adult subgroup, mtDNA defects accounted for $54 \%(14 / 26)$, which is lower than the previously estimated $70-75 \%$, possibly as a consequence of the relatively small number of adult patients in our cohort[29, 30]. As shown in Table 1, most mtDNA patients carried LHON and MIDD/MELAS causing mutations, which was also likely based on the clinical presentation. Both MIDD and MELAS symptoms were caused by the m.3243A>G mutation, in line with what has been reported before[31]. Yet, as indicated by the heteroplasmy levels (24-41\% in MELAS and $7-40 \%$ in MIDD), variation in symptom manifestations among these patients could not solely be explained from the differences in heteroplasmy level, making prognostic predictions challenging.

Using WES, we identified a disease causing variant in an additional $49 \%$ of the patient cohort, where we applied a variant selection strategy fitting the presumed genetic mode of disease inheritance. In a group with likely inherited disease (consanguineous and/or multiple patients), filtering for recessive mutations solved $70 \%$ of the patients. A group of single non-consanguineous patients was also screened for heterozygous mutations in known (OMIM) AD disease genes, which resulted in an overall genetic diagnosis in $55 \%$ of the patients, of which $16 \%$ of the identified variants were de novo. To reduce the huge amount of potential dominant mutations, we have restricted our heterozygous variant selection to OMIM-reported dominant disease genes, and therefore might have missed some de novo cases. This could be overcome by WES trio-analysis, including also the parents of the patient. Additionally, the higher likelihood of an inherited genetic cause, the availability of homozygosity mapping data and of exome data of affected siblings might have contributed to a higher diagnostic yield in the first group. Our strategy did not result in large differences in diagnostic yield between primary mitochondrial patients (MD) and patients from the differential diagnostic procedure (DD). 
In an additional $7 \%$ of the WES cohort we found a genetic defect with a clear lead to the patient's phenotype (data not shown). For these cases, further laboratory testing or patient screening should reveal which genes can be classified as disease causing.

In $84 \%$ of the MD patients, symptoms were caused by a gene with a reported mitochondrial function or localization. As expected, this was significantly lower in the DD patient group (15\%). Our results showed that in patients with primary mitochondrial disease, most genetic defects were associated with mitochondrial protein metabolism and OXPHOS function, where a majority of the OXPHOS defects were found in complex I genes. In line with previously reported complex I deficient patients, we found that defects in NDUFS7, NDUFA12 and NDUFAF5 caused Leigh-syndrome or a Leigh-likephenotype[32]. Although NDUFV2 defects have also been reported in association with Leigh syndrome[33], our patient exclusively expressed symptoms of white matter degeneration (encephalopathy). Also, we identified TMEM126A as an underlying cause of isolated complex I deficiency, therewith supporting co-migration studies that characterized TMEM126A as an early assembly factor of complex I[34]. Patients with mtDNA abnormalities carried mutations in RRM1, RRM2B, c10orf2 and DNA2, which were all functionally related to mtDNA maintenance, and presented as the third largest group. Interestingly, we also found the functionally uncharacterized c19orf12 gene associated with mtDNA depletion.

In $5 \mathrm{MD}$ patients, we identified a causal gene for which no direct mitochondrial function or localization has been reported. This included the functionally uncharacterized IER3IP1 gene and 4 genes involved in different cellular processes (SLC19A3, SLC16A2, IARS, CHRNE). In two families with classical Leigh-syndrome we identified a defect in the thiamine transporter SLC19A3, where despite a decrease in mitochondrial oxygen consumption, complex activities seemed normal in muscle and skin tissue[35]. Thiaminediphosphate, the active form of thiamine, has been reported as an essential cofactor for several mitochondrial enzymes, including the pyruvate dehydrogenase complex[36]. A second transmembrane transporter defect was found in the thyroid hormone (T3/T4) transporter SLC16A2, in a patient with Allan-Herndon Dudley syndrome, which resulted in a $\mathrm{CII}$ and $\mathrm{CIV}$ deficiency. Also here mitochondrial dysfunction seems to be a secondary effect of a transport deficiency, where triiodothyronine (T3) has been suggested as an important regulator of mitochondrial activity[37]. Furthermore, we found a mutation in the cytoplasmic located aminoacyl-tRNA synthetase IARS to cause complex I deficiency in skin and muscle tissue of a patient with a multi-system disorder, and a defect in the acetylcholine receptor subunit epsilon CHRNE associated with CII deficiency, COX negative muscle fibres and abnormally shaped muscle mitochondria in CPEO with myopathy. The genetic defects in these patients are therefore important to further characterize the underlying processes and clarify their role in mitochondrial disease.

$32 \%$ of the disease-causing genes identified in our MD-patients were not included in the MitoCarta database at the moment of identification, and could therefore be missed with panel-based sequencing approaches. In addition to the 5 genes described above, 
this involved several genes with a reported function in mitochondrial metabolism, indicating that it can be difficult to keep databases or panels up to date with recent discoveries. Also, the contribution of non-mitochondrial disease genes to complex multi-genic mitochondrial disease might be missed when applying targeted sequencing methods, as illustrated by two of the mitochondrial patient cases, in which defects in more than a single gene were involved. Our data shows that WES is the most comprehensive and unbiased approach to establish a genetic diagnosis in mitochondrial disease, able to fully resolve complex multi-genic disease manifestations. To avoid incidental findings in a diagnostic setting one might consider pre-screening WES data with virtual gene panels; however, in heterogeneous disorders, this can easily result in inefficient and sequential use of different virtual gene panels, and still miss the complete picture in multigenic disorders. Obviously, a disadvantage of WES in diagnostics settings will be the identification of novel, non-reported gene defects that often require extensive experimental setups to validate a possible role in mitochondrial disease. Embedding within or contact with a specialized research group should therefore be preferable. Identification of the genetic defect is not only important for diagnostics, but also for therapeutic interventions. Patients with SLC25A32, SLC19A3 and TMEM126B defects showed improvement upon treatment with respectively riboflavin, biotin/thiamine and high fatdiet[35]. Yet, despite the significant improvements in the genetic diagnosis of mitochondrial disorders, the development of novel therapies is lagging behind. 


\section{REFERENCES}

1. Rotig, A. and A. Munnich, Genetic features of mitochondrial respiratory chain disorders. J Am Soc Nephrol, 2003. 14(12): p. 2995-3007.

2. McFarland, R., R.W. Taylor, and D.M. Turnbull, A neurological perspective on mitochondrial disease. Lancet Neurol, 2010. 9(8): p. 829-40.

3. Hellebrekers, D.M., et al., PGD and heteroplasmic mitochondrial DNA point mutations: a systematic review estimating the chance of healthy offspring. Hum Reprod Update, 2012. 18(4): p. 341-9.

4. Thorburn, D.R. and H.H. Dahl, Mitochondrial disorders: genetics, counseling, prenatal diagnosis and reproductive options. Am J Med Genet, 2001. 106(1): p. 102-14.

5. Calvo, S.E. and V.K. Mootha, The mitochondrial proteome and human disease. Annu Rev Genomics Hum Genet, 2010. 11: p. 25-44.

6. Prokisch, H. and U. Ahting, MitoP2, an integrated database for mitochondrial proteins. Methods Mol Biol, 2007. 372: p. 573-86.

7. Alston, C.L., et al., The genetics and pathology of mitochondrial disease. J Pathol, 2017. 241(2): p. 236250.

8. Hui, J., et al., Decreased activities of mitochondrial respiratory chain complexes in non-mitochondrial respiratory chain diseases. Dev Med Child Neurol, 2006. 48(2): p. 132-6.

9. Fan, H., et al., Detection of common disease-causing mutations in mitochondrial DNA (mitochondrial encephalomyopathy, lactic acidosis with stroke-like episodes MTTL1 3243 A>G and myoclonic epilepsy associated with ragged-red fibers MTTK $8344 \mathrm{~A}>\mathrm{G}$ ) by real-time polymerase chain reaction. J Mol Diagn, 2006. 8(2): p. 277-81.

10. Wang, J., et al., Analysis of mitochondrial DNA point mutation heteroplasmy by ARMS quantitative PCR. Curr Protoc Hum Genet, 2011. Chapter 19: p. Unit 196.

11. Finsterer, J., et al., EFNS guidelines on the molecular diagnosis of mitochondrial disorders. Eur J Neurol, 2009. 16(12): p. 1255-64.

12. van Eijsden, R.G., et al., Chip-based mtDNA mutation screening enables fast and reliable genetic diagnosis of OXPHOS patients. Genet Med, 2006. 8(10): p. 620-7.

13. McCormick, E., E. Place, and M.J. Falk, Molecular genetic testing for mitochondrial disease: from one generation to the next. Neurotherapeutics, 2013. 10(2): p. 251-61.

14. Tang, S. and T. Huang, Characterization of mitochondrial DNA heteroplasmy using a parallel sequencing system. Biotechniques, 2010. 48(4): p. 287-96.

15. Xie, H.M., et al., Mitochondrial genome sequence analysis: a custom bioinformatics pipeline substantially improves Affymetrix MitoChip v2.0 call rate and accuracy. BMC Bioinformatics, 2011. 12: p. 402.

16. Wong, L.J., et al., Compensatory amplification of mtDNA in a patient with a novel deletion/duplication and high mutant load. J Med Genet, 2003. 40(11): p. e125.

17. He, L., et al., Detection and quantification of mitochondrial DNA deletions in individual cells by real-time PCR. Nucleic Acids Res, 2002. 30(14): p. e68.

18. Cui, H., et al., Comprehensive next-generation sequence analyses of the entire mitochondrial genome reveal new insights into the molecular diagnosis of mitochondrial DNA disorders. Genet Med, 2013. 15(5): p. 388-94.

19. Huang, T., Next generation sequencing to characterize mitochondrial genomic DNA heteroplasmy. Curr Protoc Hum Genet, 2011. Chapter 19: p. Unit19 8.

20. Li, M., et al., Detecting heteroplasmy from high-throughput sequencing of complete human mitochondrial DNA genomes. Am J Hum Genet, 2010. 87(2): p. 237-49.

21. Wortmann, S.B., et al., Whole exome sequencing of suspected mitochondrial patients in clinical practice. J Inherit Metab Dis, 2015. 38(3): p. 437-43.

22. Choi, M., et al., Genetic diagnosis by whole exome capture and massively parallel DNA sequencing. Proc Natl Acad Sci U S A, 2009. 106(45): p. 19096-101. 
23. Li, H., et al., The Sequence Alignment/Map format and SAMtools. Bioinformatics, 2009. 25(16): p. 20789.

24. Seelow, D., et al., HomozygosityMapper--an interactive approach to homozygosity mapping. Nucleic Acids Res, 2009. 37(Web Server issue): p. W593-9.

25. Neveling, K., et al., A post-hoc comparison of the utility of sanger sequencing and exome sequencing for the diagnosis of heterogeneous diseases. Hum Mutat, 2013. 34(12): p. 1721-6.

26. Lieber, D.S., et al., Targeted exome sequencing of suspected mitochondrial disorders. Neurology, 2013. 80(19): p. 1762-70.

27. Calvo, S.E., et al., Molecular diagnosis of infantile mitochondrial disease with targeted next-generation sequencing. Sci Transl Med, 2012. 4(118): p. 118ra10.

28. Pronicka, E., et al., New perspective in diagnostics of mitochondrial disorders: two years' experience with whole-exome sequencing at a national paediatric centre. J Transl Med, 2016. 14(1): p. 174.

29. Schaefer, A.M., et al., Prevalence of mitochondrial DNA disease in adults. Ann Neurol, 2008. 63(1): p. 359.

30. Gorman, G.S., et al., Prevalence of nuclear and mitochondrial DNA mutations related to adult mitochondrial disease. Ann Neurol, 2015. 77(5): p. 753-9.

31. de Wit, H.M., et al., MIDD or MELAS : that's not the question MIDD evolving into MELAS : a severe phenotype of the m.3243A>G mutation due to paternal co-inheritance of type 2 diabetes and a high heteroplasmy level. Neth J Med, 2012. 70(10): p. 460-2.

32. Rahman, S. and D. Thorburn, Nuclear Gene-Encoded Leigh Syndrome Overview, in GeneReviews(R), R.A. Pagon, et al., Editors. 1993, University of Washington, Seattle. GeneReviews is a registered trademark of the University of Washington, Seattle: Seattle WA.

33. Cameron, J.M., et al., Exome sequencing identifies complex I NDUFV2 mutations as a novel cause of Leigh syndrome. Eur J Paediatr Neurol, 2015. 19(5): p. 525-32.

34. Wessels, H.J., et al., Analysis of 953 human proteins from a mitochondrial HEK293 fraction by complexome profiling. PloS one, 2013. 8(7): p. e68340.

35. Gerards, M., et al., Exome sequencing reveals a novel Moroccan founder mutation in SLC19A3 as a new cause of early-childhood fatal Leigh syndrome. Brain, 2013. 136(Pt 3): p. 882-90.

36. Ortigoza-Escobar, J.D., et al., Thiamine transporter-2 deficiency: outcome and treatment monitoring. Orphanet J Rare Dis, 2014. 9: p. 92.

37. Wrutniak-Cabello, C., F. Casas, and G. Cabello, Thyroid hormone action in mitochondria. J Mol Endocrinol, 2001. 26(1): p. 67-77. 

Chapter

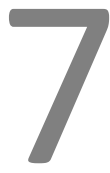

General discussion 

The development of next generation sequencing (NGS) technologies has revolutionized the field of genetics and characterization of the genetic basis of many genetic and complex diseases. Huge progress has been made towards understanding the cause of neurological diseases in particular. In this discussion, I summarize the major contributions of NGS to the study of neurological diseases with a particular focus on mitochondrial diseases. I will not only highlight the possibilities, but also the particular pitfalls in NGS technology and I will discuss both clinical and research applications of these sequencing platforms for neurological disorders. Tremendous advances in disease gene identification emphasizes the need for model systems that provide experimental evidence for the underlying mechanism of pathogenesis or for the way specific variants damage proteins and lead to disease. Therefore, the second part discusses several model systems, in vitro and in vivo, which are utilized in studying genetic neurological disorders. The advantages, limitations and relevant recapitulation of the essential features of the human neurological diseases will be discussed for each model.

\subsection{CLINICAL AND GENETIC HETEROGENEITY OF NEUROLOGICAL AND MITOCHONDRIAL DISEASES}

The estimated number of rare genetic diseases is 7000 , affecting at least 1 in 50 individuals [1]. Half of these diseases display dysfunction of the central or peripheral nervous system $[2,3]$. Clinical symptoms include, among others, brain malformation, neuropathies, encephalopathies, myopathies and muscular dystrophies, movement disorders, and dementias. The genetically heterogeneous nature of neurological and mitochondrial diseases makes identifying the disease-causing mutations highly challenging and until recently only possible in a minority of cases. For example, hereditary spastic paraplegia is caused by $>50$ disease genes, and $>300$ disease genes have been associated for ataxia [2]. Mutations in more than 60 genes, both nuclear and mitochondrial DNA encoded, have been reported to cause one mitochondrial disease, Leigh syndrome, only, still explaining only half of all cases [4]. The total number of genes potentially involved in mitochondrial disease adds up to over 1500.

Mitochondrial diseases are unique that the genetic basis involves 2 genomes, as both the mtDNA and the nuclear genome can be involved. mtDNA mutations can manifest as a number of well-described syndromes (Leigh syndrome [LS], myoclonic epilepsy with ragged-red fibers [MERRF], mitochondrial encephalomyopathy with lactic acidosis and stroke-like episodes [MELAS], Kearns-Sayre syndrome [KSS], chronic progressive external ophthalmoplegia [CPEO] or neurogenic weakness with ataxia and retinitis pigmentosa [NARP]) [5], but patients can also present with other more common and lessspecific symptoms, like cardiomyopathy, optic atrophy, pigmentary retinopathy, external ophthalmoplegia, ptosis, proximal myopathy and exercise intolerance, deafness and diabetes mellitus [5]. Moreover, the clinical features are further complicated by re- 
duced penetrance, varying age-of-onset, variable expressivity and atypical presentations.

Mitochondrial patients with mutations in nuclear genes also display a broad variety of clinical and biochemical manifestations and new mutations in known and unknown genes are frequently reported. The identification of novel gene defects and genes is not only essential for diagnostic purposes, but also to elucidate disease mechanisms and possible therapeutic targets, and to obtain a complete picture of the genes and pathways underlying neurological and mitochondrial disorders. The development of next generation sequencing (NGS) technology has enabled novel strategies, like sequencing large gene panels, whole exome sequencing (WES) or even whole genome sequencing (WGS), to achieve this. NGS has led to a paradigm shift in the diagnostics of genetically heterogeneous disorders, which was phenotype-based and is becoming genotypebased.

\subsection{CANDIDATE GENE APPROACH USING GENE PANELS}

Gene panels offer mutation screening of large number of genes associated with a specific phenotype in a single reaction [6]. This is particularly useful in genetic disorders, in which a limited number of genes explains the vast majority of cases or as a prescreening for genetically more heterogeneous diseases. Such disease categories include inherited neuropathy, myopathy, neuromuscular junction diseases, and mitochondrial diseases [7-10]. Gene panels have several benefits over WES or WGS. Sequencing with molecular inversion probes (MIPs) enable the gene panels to be used as an initial relatively costeffective approach [11]. Depth of sequencing is typically higher for gene panels and the design is such that every exon is present at comparable coverage, implying that this approach is less likely to miss a mutation in the genes tested. Moreover, as smaller gene panels require less sequence capacity, more samples can be pooled in a single sequence run making gene panels even cheaper and faster than WES and WGS. Given that panels contain disease specific genes, they are unlikely to generate unsolicited findings. Also, the number of variants of unknown significance (VUS) detected by a panel approach is considerably lower than WES or WGS [12]. Gene panels yield a diagnostic rate similar to that of WES for diagnosing hereditary polyneuropathy and epilepsy [8, 13, 14]. Specifically, among patients with inherited neuropathy and young age at onset ( $<40$ years), the diagnostic rates are approximately $30 \%$ for both WES and gene panels, despite the fact that WES sequences more than 20,000 genes while the neuropathy panel only includes 192 genes $[8,13]$. This implies that the 192 genes cover all causes of monogenic neuropathy at young age, but that in $70 \%$ of the cases, the cause might not be a severe, rare pathogenic mutation in the exome, which is the filtering strategy applied. Environmental causes could be involved as well, probably interacting with more common ge- 
netic variants, which would require another approach, which might not yet be feasible at the level of the individual patient.

The main limitation for the use of gene panels instead of WES and WGS is that no new genes will be identified. Despite tremendous advances in the genetic field, sequence variations in known genes explain only a part of the genetic causes of neurological disease [15]. A recent study using gene panel mutation screening in patients with hereditary spastic paraparesis detected the genetic cause in $25 \%$ of patients who were negative for mutations in the most common gene associated with spastic paraparesis [16]. A diagnosis was established for $22 \%$ of 102 patients with suspected mitochondrial disorders by sequencing the mitochondrial genome (mtDNA) and the exons of 1,598 nuclear-encoded genes implicated in mitochondrial biology, mitochondrial disease, or monogenic disorders with phenotypic overlap [17]. A similar diagnostic rate of $24 \%$ was reported for 42 patients with clinical and biochemical evidence of mitochondrial disease by performing "MitoExome" sequencing of the mitochondrial DNA (mtDNA) and exons of 1000 nuclear genes encoding mitochondrial proteins [18]. In another study, a gene panel of 447 nuclear genes encoding proteins involved in OXPHOS complexes, genes inducing secondary mitochondrial dysfunction and genes that cause diseases which mimic mitochondrial disorders were tested in 148 patients. Variants already known to cause disease in 12 genes were detected 13 patients (8\%) and rare or novel variants of unknown significance were identified in 45 additional genes for various metabolic, genetic or neurogenetic disorders in 67 patients (45\%) [19]. A diagnostic yield of $\sim 24 \%$ using a panel-based approach is much less than that of WES in a cohort of patients with suspected mitochondrial diseases in our study (50\%) and other studies [20, 21]. There are several explanations for the lower yield. First, not all mitochondrial genes are listed in MitoCarta or other databases, on which gene panels are based. Our study showed that $31 \%$ of the disease-causing genes in mitochondrial WES-patients were not included in the MitoCarta database at the time of discovery and as mentioned before these novel genes could not be detected by a panel based approach. A phenotype may also be caused by multiple genetic defects and a panel may not cover all of them. Finally, a subset patients may not actually have primary mitochondrial disease, despite clinical and biochemical features used in the clinical diagnostic algorithms.

Panels also have the risk of becoming quickly out of date as new genes are being identified or as atypical features of other disorders may overlap with a panel's particular indication. As a result, the causal gene defect might not be identified hampering possibility of treatment and genetic counseling. An alternative is using a bioinformatics panel, based on whole exome data, allowing 2-step-procedure on a single exome data set. First only candidate genes are being analyzed (panel) and, if negative, secondly all genes within the exome by WES. When clinical presentations are nonspecific and involve multisystem symptoms, a situation in which it is difficult to choose a specific panel, WES or WGS should be employed immediately. 


\subsection{WHOLE EXOME SEQUENCING}

WES is being increasingly applied as a first test to establish a genetic diagnosis in patients with neurological and mitochondrial disorders. WES interrogates nearly all protein-coding and flanking intronic regions in the human genome which although representing less than $2 \%$ of DNA, contains the majority of known disease-related variants [22]. WES has allowed for rapid and accurate detection of mtDNA mutations, and more recently copy number variations $[23,24]$. In addition to targeted sequencing of the mtDNA, the mtDNA sequence can also be extracted from exome sequencing data, be it with a lower coverage [25]. Given the relative abundance of mtDNA, the mtDNA sequence is one of the most common detected aspecific sequencing data such as exome, whole genome and RNAseq [26]. A significant number of reads will align to the mitochondrial genome (around 1-5\%), even when the mitochondrial genome is not the intended sequencing target [26].

WES also has its limitations mainly due to incomplete or low coverage of certain exons or even whole genes due to capture probe design. Designs with higher density seem to be more efficient, providing better uniformity and more complete coverage. However, this approach appears inefficient when trying to fully capture all known genes including those not yet associated with disease. Problems occur in extreme GC-rich regions which are prone to low coverage due non-specific binding during capture and poor amplification [27-30]. It is estimated that, as a result of these technical limitations, $\sim 10 \%$ of the exome is poorly covered [7]. Other reasons for not reaching a genetic diagnosis are poor annotations, and shortcomings of variant calling tools with respect to structural variation and insertions/deletions, inaccurate phenotyping, non-genetic disease or sample errors. Furthermore, WES does generally not cover untranslated regions, enhancers, and long-noncoding RNAs that may be of functional relevance, although these could be added to the capture kits. Regulatory mutations that reside outside of coding regions will escape detection by WES. The success yield of WES in a clinical setting will increase, in the near future, as all the relevant genetic regions will be interrogated at sufficient coverage.

As WES identifies a huge number of variants, filtering approaches are needed to reduce the number of genes for further investigation. On average, more than 50, 000 variants are detected per individual through WES. Filter strategies in severe genetic disease are usually based on searching for rare, highly harmful variants that fit the genetic model. After excluding common variants deposited in public and in-house databases, UTR variants, non- splice site related intronic variants, synonymous variants, usually only 200 variants are left. The damaging-effect of the variants on the protein are based on prediction tools e.g SIFT and Polyphen2. However, these predictions are not always consistent between these tools. The variants should also fit with the presumed mode of inheritance, generally autosomal recessive or de novo disease for mitochondrial neurological disease. In consanguineous patients, selecting genes from re- 
gions of homozygosity has contributed to reducing the number of variants and a higher diagnostic yield. For single non- consanguineous patients who are not homozygous or compound heterozygous for a pathogenic mutation, WES for both parents and the child should be performed to detect de novo mutations.

Over the past 2-3 years, several studies have described WES as a diagnostic tool in clinical genetic laboratories [31-33]. The Baylor College of Medical Genetics Laboratory reported on a $25 \%$ molecular diagnostic rate for 250 probands with a range of phenotypes, in which $80 \%$ were children with neurological phenotypes [31]. A similar diagnostic rate of $22-31 \%$ was reported for over 814 patients by Hane Lee et al in another clinical laboratory at the University of California, Los Angeles, Clinical Genomics Center [33]. A diagnostic yield of 59\% was found in pediatric patients with a mitochondrial disorder, screening both the mtDNA and the exome [21]. Another WES study was performed in 109 pediatric and young adult patients with mitochondrial disease, establishing a genetic diagnosis in 39\% of the patient cohort (after exclusion of mtDNA mutations) [20]. In our study, we used a next-generation sequencing strategy in a cohort of 86 patients with primary mitochondrial disease and 33 patients, where a mitochondrial cause is part of the differential diagnosis. In $70 \%$ of the patients, we identified a disease causing genetic defect, of which $20 \%$ was solved by mtDNA sequencing and $50 \%$ by subsequent WES analysis. Our strategy did not result in large differences in diagnostic yield between primary mitochondrial patients and patients from the differential diagnostic procedure, most likely because a genetic basis was based on consanguinity or on multiple sibs in a family. WES has been highly successful, by increasing the diagnostic yield, from $20 \%$ to $70 \%$ in a well-characterized group of patients. The differences in diagnostic yield of WES probably reflects a number of factors including analysis strategy (singleton or trio), type of disorder, age of presentation, onset of symptoms, clinical presentation, and the likelihood that the disease is genetic, caused by a single gene or multiple genes. A broad range of phenotypic presentations and a large number of possible disease genes increase the complexity of interpreting genetic data. Also, the quality of the data and data analysis likely contributes to this. Our results show that WES should be implemented as diagnostic test for mitochondrial disorders, without the necessity of prior gene panel analysis.

\subsection{RNA-SEQ AND WHOLE GENOME SEQUENCING}

While WES has many advantages over WGS, including the lower cost, a yield of up to $70 \%$ by screening less than $2 \%$ of the genome, and an easier analysis of exonic variants, it is also clear that WES will miss disease-causing variants, including variants in the noncoding regions and introns, in regulatory regions such as promoters and enhancers, and structural variants. In the Human Gene Mutation Database [34], at least 1.4\% of the disease-causing variants are in regulatory sequences and about $7.5 \%$ are structural 
variants. Neurodevelopmental and psychiatric disorders represent two particularly large groups of diseases often linked to structural genome changes [35]. However, there is also a growing list of other neurological disorders including Parkinson, Alzheimer, Hereditary Leukodystrophies, Spinal Muscular Atrophy, Charcot-Marie-Tooth Disease, Neurofibromatosis Type 1 [35-37] which are caused by chromosomal microdeletions, microduplication, or other genomic rearrangements. Higher average coverage is required for WES compared to WGS, because of the variable success of capturing different regions and because of "allelic imbalance" where one allele is preferentially captured over the other.

Whole-genome sequencing (WGS) has the potential to identify nearly all forms of genetic variation [38]. Several studies have demonstrated the advantages of WGS for mutation detection [39-42]. Moreover, WGS covers the whole genome at more consistent coverage than WES. As a result, it is possible that WGS identifies mutations in coding regions which were not detected using WES. Whole genome sequencing has been successfully applied to various neurological diseases including in a family with a rare form of autosomal recessive Charcot-Marie-Tooth disease [39], early-onset epilepsy [43] and sensory and motor neuropathy with microcephaly [44]. WGS analyses of paediatric populations has shown identification of clinically relevant variants in $\sim 60 \%$ of patients with intellectual disability [45]. In the future, the results of WES or WGS can be re-assessed when additional genes are linked to diseases or new pathways are found.

The fact that the majority of pathogenic mutations are found in coding regions, as well as the substantial extra cost and bioinformatic challenges faced with handling the larger WGS data have so far prohibited a broad use of WGS. However, with decreasing cost of sequencing and the advancement in computing, we expect that WGS will eventually replace WES for a comprehensive evaluation of undiagnosed patients with neurological and mitochondrial diseases. And in the not so far away future, WGS might be carried out on population basis. In the US the genome of $1,000,000$ citizens is sequenced as a first step to achieve genome-based precision medicine [46].

In addition to WES and WGS, which generate a large number of variants required to prioritize the candidate disease genes, transcriptome using NGS technology (RNA-Seq) is becoming increasingly popular. This approach involves direct sequencing of transcripts in a high-throughput and quantitative manner at low cost [47]. RNA-Seq has considerable advantages for the detection of mutations with a direct negative effect on the RNA (splice defect, allelic imbalance, RNA degradation), wherever they are in the genome [48]. RNA-Seq enabled a diagnosis in patients with mitochondrial and neurological disorders where WES had no answer. According to a study led by researchers in Munich, RNA-Seq provided diagnoses for $10 \%$ of patients who had undergone WES but did not receive a genetic diagnosis [49]. In another study, Cummings et al used RNA-Seq as a complementary diagnostic tool in a cohort of 50 patients with undiagnosed muscle disorders. With this approach, they were able to provide a diagnosis for $35 \%$ of the 
patients [50]. We expect that RNA-Seq will become an essential companion of genome sequencing to address undiagnosed cases of neurological and mitochondrial disorders.

\subsection{IMPACT OF NEXT GENERATION SEQUENCING ON PREVENTION AND TREATMENT}

The introduction of WES and WGS in the clinic has led to the identification of many novel disease genes, which in a number of cases has enabled novel treatments or revealed possible therapeutic targets [51]. Although treatments are not possible for the majority of patients with neurological disorders, patients benefit from early and accurate diagnosis as this, in some cases, enables treatment managing and/or relieving symptoms and preventing further complications. Also, transmission to subsequent generations can be prevented by prenatal or preimplantation genetic diagnosis. In future, whole genome sequencing of fetal cell-free DNA in maternal plasma or serum [52], would allow non-invasively sequencing of the entire fetal genome which may ultimately facilitate the diagnosis of all inherited and de novo genetic disease during early pregnancy $[53,54]$.

A successful treatment based on the gene defect, has been developed for a few gene defects, like the specific form of the infantile-onset, Leigh-like disease caused by mutations in the SLC19A3 gene. Immediate treatment with a high-dose of thiamine and, possibly, biotin, prevents fatal Leigh syndrome [55]. Mutations in ACAD9 are associated with exercise intolerance [56], with infantile hypertrophic cardiomyopathy, encephalopathy and lactic acidosis $[57,58]$. A reduction of complex I enzymatic activity was observed in all patients. Most ACAD9 mutant cells and patients respond to riboflavin treatment, with partial correction of complex I deficiency and clinical improvement [56, 57]. Coenzyme Q10, or CoQ10, supplementation has been widely used for treatment due to its well documented safety. Adverse effects are rare even at a high concentration [59]. CoQ10 therapy has shown efficacy not only in patients with primary CoQ10 biosynthesis deficiencies, but also in several other mitochondrial disorders although to a lesser degree [60]. Some Leigh patients with primary CoQ10 deficiencies have almost complete recovery or marked improvement of neurologic deficits when treated with CoQ10 [4].

Several other studies in non-mitochondrial disease reported novel treatments based on novel genes identified by WES. In one of these studies, WES uncovered 10 probands ( $8 \%$ of the cohort) with mutations in known disease genes that led to a change in the diagnosis of the patients and in some cases resulted in new treatments [61]. This included a family with four patients presenting early- onset intellectual disability and mild dysmorphic features [61]. By WES a homozygous mutation was detected in the MAN2B1 gene, establishing the diagnosis of alpha-mannosidosis, a rare lysosomal storage disorder, instead of the initial diagnosis of recessive intellectual disability. Alpha - 
mannosidosis can be cured with transplanting hematopoietic stem cell which had not been considered prior to the new genetic diagnosis [61]. Without treatment, alphamannosidosis follows a degenerative course including skeletal malformation, developmental delay, hearing loss and recurrent otitis or other infections [62], which can be prevented by early intervention before these signs occur [63]. Therefore, early diagnosis is crucial in order to prevent irreversible cognitive and skeletal impairment. In a different study, including 12 individuals with unexplained and apparent genetic conditions, WES revealed a likely genetic diagnosis in 6 patients. For two individuals, the identification of the genetic cause, led to new treatment and/ or surveillance that would not have been proposed before. Without finding the genetic cause, improper medications could potentially even worsen clinical symptoms and increase the severity of the disease [64]. These studies highlight the importance of early genetic diagnosis not only for having the correct diagnosis but also successful therapeutic intervention.

\subsection{FUNCTIONAL MODELS}

Model systems, such as rodents, especially mice as one of the most commonly used models, and different species, including zebrafish (Danio rerio), Drosophila, Caenorhabditis elegans, but also cellular and in silico models have provided valuable tools for experimental investigation. They have been essential in understanding the pathogenicity of a particular variant, disease-associated genes, phenotype-genotype correlations, fundamental mechanisms underlying human neurological and mitochondrial disorders. Consequently, models contribute to improved diagnosis and treatment. Most in vivo and in vitro models are easy to manipulate and provide large enough groups to achieve statistical power. Additionally, animal models offer the opportunity to investigate the developing embryo and all stages of disease pathology in a whole organism, which can provide fundamental insight in the underlying pathophysiological mechanism. This is often not possible in patients as access to tissues or organs is limited during life and postmortem samples might reflect the end-stage of the disease or could reveal secondary pathology $[65,66]$. Recently, there have been new and exciting developments in the field of functional models for human disorders with the introduction of iPSC technology to generate pluripotent cells. These iPSCs (induced pluripotent stem cells) can be differentiated into many different cell types or tissue, which allows studying the genetic defect in the cells or tissues where the disease manifests. This will allow more accurate characterization of the pathophysiological mechanism and the possibility to test personalized therapeutic interventions [67]. Also, computational models/simulations are getting more common and important as these enable in silico experiments that would be difficult or impossible to conduct in cells or model organisms. During the past decade, Computational Neurogenetics models [68] have been used to study interactions between genes and their effects on neuronal functions and the brain activity as a 
whole. In a series of studies, this approach was used to investigate the effect of a specific gene knockout on gene regulatory network and therefore the dynamics of a neuronal network [68]. Computational models has been applied to neurological disorders including Parkinson's disease and movement disorders, Huntington's disease, Alzheimer's disease [69].

Functional models for genetic diseases can be created by mutating the gene of interest. This can be done in several ways, either a-specifically using chemical or insertional mutagens. A number of different methods have been developed for knocking out genes to characterize genes with a specific function as part of a genome-wide screening approach, or directed at specific genes, by in vitro mutagenesis [70], morpholinos [71], TILLING [72], zinc-finger nucleases [73], TAL-effector endonucleases [74]. Most recently, the CRISPR-Cas9 system, which has become the preferred and most efficient strategy to create or correct specific mutations in genes [75]. Whatever approach is being used and which model is being preferred, largely depends on the question to be answered. A general rule of thumb is to select the simplest model. For example, to demonstrate the pathogenicity of a mutation in a known or novel gene in mitochondrial disease, where a biochemical defect is responsible for the clinical manifestations, usually a complementation assay in fibroblasts suffices. Fibroblasts with characterized mutations are invaluable tools for studying the pathomechanism of mitochondrial disorders and deciphering new biochemical pathways and interactions. Patient's fibroblasts provided a unique tool for examining, for example, of various aspects of the human mitochondrial translation system and mtDNA content [76]. The functional role of mitochondrial ribosomal proteins in the structure and assembly of the large (MRPL44) and the small subunits (MRPS22, MRPS16) of the human mitochondrial ribosome was shown by studying patients' fibroblasts $[77,78]$. Mitochondrial membrane organization and structure, cardiolipin and phospholipid remodeling were demonstrated to be important largely based on fibroblasts from patients with Barth, MEGDEL and Senger syndromes caused by mutations in TAZ, SERAC1 and AGK genes, respectively [79-81].

However, to explain the clinical manifestations in patients the chosen model can vary and for example for developmental defects the zebrafish model is preferred, whereas for adult-onset neurological disease and cognitive decline, mice seem more appropriate. As the same genetic defect might manifest differently among patients, cheap outbred models with varying genetic background can be chosen to study the impact of the genetic background or environment on disease manifestation. Each model has its own advantages and limitations and these will be discussed, for the most commonly used models in neurological and mitochondrial disease in more detail in this section. I will focus on the possibility of each model to classify mutations and genes- and on the similarities with humans to be able to explain the pathology. 


\subsection{MOUSE MODEL}

One of the best-known animal models used over time are mice (Mus musculus), which were first used for the study of respiration in the $17^{\text {th }}$ century. By 1909, the first inbred strain, DBA, was produced which marked the era of modern mouse genetics. This mouse strain evolved into a genetic tool that is important for mapping and identifying genes with strong implications for human biology and disease. Since then, hundreds of inbred strains have been developed. In 1929, CC Little founded the Roscoe B Jackson Memorial Laboratory, a world-wide resource for mouse model research [82]. Today, mice are the most common mammalian model used to study a wide variety of diseases including neurological disorders, metabolism, development and many others [83]. Although the divergence of humans and mice from a common ancestor occurred about 80 million years ago, mice share the majority of their genes with humans, and on average, the protein-coding regions of the human and mouse genomes are approximately $85 \%$ identical (range 60-99\%) [84]. Actually, almost all human genes can functionally replace the corresponding endogenous gene of the mouse. Thus, a genetic defect affecting a protein important for the mouse nervous system is likely to resemble a neurologi$\mathrm{cal} /$ neurodegenerative phenotype in humans.

Mice have several advantages to study mammalian diseases. Firstly, inbred strains were traditionally created by mating brother-sister mice over 20 generations, which resulted in offspring that were genetically identical [85]. By using inbred strains, the differences in the genetic background can be controlled. This allows examining the effects of a mutation among different mice, each of them having the same genetic background. A drawback is that the genetic background in inbred mice might diverge from human and the clinical manifestations in the mice might therefore not reflect the clinical manifestations in patients. Often researchers have to perform multiple experiments to find the best matching inbred strain. Still, inbred mice strains have become a powerful genetic tool which plays an important role in characterizing genes and gene defects in human genetic disease, as illustrated in the examples given below. Secondly, an extensive toolbox has been developed to create the preferred transgenic mouse model, including knock-out mice, in which the gene of interest is deleted or a nonsense mutation introduced, and knock-in mice, in which a specific nucleotide is substituted, inserted or deleted to study the causal role of mutations in human pathological conditions. Genome-wide approaches of these technologies have been used to create a library of mutants in which every mouse gene is disrupted [86]. Phenotyping the mutants will reveal novel genotype-phenotype correlation, leading to the first functional map of a mammalian genome. Although mice have given important contributions as a model of human research, there are also some drawbacks for using mice. For example, they are difficult to manipulate embryonically, expensive to maintain, and their considerable costs limits their use in large scale genetic studies and compound screenings. 
A few successful examples of mouse models in neurological and mitochondrial diseases are:

- Amyoptropic lateral sclerosis (ALS). SOD1 mutations in humans are associated with familial forms of amyotropic lateral sclerosis (ALS) [87]. Transgenic mice containing human mutants, G93A, G37R and G85R, in the SOD1 gene demonstrate progressive motor-neuron degeneration similar to ALS patients [88].

- Leigh syndrome. In humans, NDUFS4 mutations are associated with Leigh syndrome, a progressive neurodegenerative disorder [89]. The NDUFS4 knock-out mice developed, like human patients, isolated $\mathrm{Cl}$ deficiency, exhibiting a tissue-specific, with liver and CNS being the most affected tissues [90]. Moreover, these mice developed the typical severe Leigh phenotypes characterised by encephalopathy, ataxia, blindness, elevated serum lactate. However, knock-out mice mature to adulthood and are apparently healthy up to $\sim 5$ wk of age, when ataxic signs started and progressed until death at $\sim$ wk whereas most patients with homozygous NDUFS4 mutations die at infancy. This could suggest some partial function of complex I in knock-out mice or differences in genetic background [90]. Remarkably, a tissue-specific knock-out of NDUFS4, exclusively expressed in neurons and glia, resulted in the same phenotype as the full-body knockouts [91], suggesting also tissue-specific characteristics, despite an expression of the gene defect in all genes. This occurs more often in mitochondrial disease and is still poorly understood. Both the full-body knockout and tissue specific knock out mouse models are essential for understanding the pathophysiological mechanism in Leigh syndrome and for developing therapeutic interventions.

Although mice model has been highly successful to study pathophysiological mechanisms in diseases, there are also cases in which mouse models match human disorders less or not and demonstrate different phenotypes compared to humans. For example, GM2 gangliosidoses are a group of severe neurodegenerative disorders, including TaySachs and Sandhoff disease. These disorders are caused by a deficiency of glycohydrolase which accumulates GM2 ganglioside within lysosomes mainly in neuronal cells. Patients with GM2 gangliosidosis present with progressive neurodegenerative symptoms including motor deficits, progressive weakness and hypotonia, alterations of language, vision deterioration, and seizures. Mouse models of Tay-Sachs and Sandhoff diseases have been generated by targeted disruption of the causative genes in human, being HEXA (Tay-Sachs) and HEXB (Sandhoff's disease) [92-94], causing similar accumulation of GM2 gangliosides in the brain as seen in humans. While the HEXA knock out mice had no obvious neurological or behavioral deficits, the HEXB mice displayed rapid, progressive, and fatal neurodegeneration [92-94] unlike in human patients in which defects in both genes result in neurodegeneration. In this model, still the mechanism of accumulation of gangliosides in the brain can be studied, despite the different clinical outcome. To study the relation with the pathology, it might be necessary to change the genetic background of the mice $[95,96]$ or to investigate the underlying and maybe 
different biochemical pathways between men and mice. These differences might also reveal rescue mechanisms that could lead to novel therapeutic approaches, which demonstrate the value of both matching and non-matching phenotypes between mouse and men.

\subsection{ZEBRAFISH MODEL}

Zebrafish is an attractive vertebrate model, which has a number of advantages compared to mice. Zebrafish have a small size, external fertilization and, compared to other vertebrate models, a rapid life cycle. Moreover, the developing embryos of zebrafish are transparent enabling live monitoring of organ development. The zebrafish genome project was started at the Wellcome Trust Sanger Institute in 2001 and completed in 2013. Comparison between the human and zebrafish genome shows that of the 3,176 human genes which have been associated with disease in humans (listed in the Online Mendelian Inheritance in Man (OMIM) database), 2601 (82\%) have at least one orthologue in zebrafish. A similar comparison revealed that there is at least one zebrafish orthologue for approximately $70 \%$ of human genes [97]. The zebrafish genome consists of 25 paired chromosomes and is more complex than the human genome with more than 26,000 protein-coding genes, the largest gene set of any sequenced vertebrate [97]. Analysis of the zebrafish genome has revealed this was the result of a whole genome duplication in teleost evolution followed by massive gene loss, that did not occur in mammals [98]. Some of these duplicated genes are expressed in different tissues compared to the human orthologue and although one of the duplicates retained the original function, the other might have a new function compared to the ancestral gene [98]. This can be a disadvantage when performing functional studies in zebrafish as a mutation in one of the zebrafish paralogs may show a less severe or no phenotype compared to a mutation in the mammalian orthologue. For example, a mutation in human orthologue may be embryonically lethal, whereas a mutation in one of the zebrafish paralogs may present a less severe phenotype with viable embryo [99]. This has been shown for the PAX6 gene, which encodes an evolutionarily highly conserved transcription factor, with paired- and homeodomain DNA-binding regions. It is required for eye, brain, olfactory system and pancreas development from Drosophila to humans $[100,101]$. In humans, PAX6 mutations are usually embryonic lethal, or some cases have survived with no or small eyes and central nervous system disorders [102, 103]. Zebrafish have two Pax6 genes, Pax6a and Pax6b. Zebrafish Pax6b missense mutations in the homeobox presented milder eyes defects and can be grown into adulthood with full fertility, suggesting the other co-orthologue Pax6a jointly fulfill the function [104].

Various experimental techniques have been developed for knockdown [105] or transgenesis [106] as well as protein overexpression which is used to study protein 
localization and function in zebrafish at the early stages of development [107]. One of the most commonly used knockdown technique in zebrafish is the antisense morpholino-mediated approach. Morpholino oligonucleotides (MOs) are nonionic DNA analogs of typically 25 morpholine bases that bind to the complementary RNA of interest [108]. There are two types of MOs, splice blocking and translation blocking. The splice blocking MOs bind and inhibit pre-mRNA processing to the mature mRNA whereas the translational blocking MOs block protein translation of the mRNA at the translational start site [105]. The biggest advantage of MOs is that phenotypes can be rapidly observed in zebrafish, though being restricted to early development. MOs have at least two significant drawbacks. First, some MOs can elicit undesirable off-target effects, producing nonspecific phenotypes. Appropriate controls with control-MOs are necessary [109], and p53 inhibition could potentially suppress off-targets effects [110]. Secondly, although morpholinos provide a stable, specific activity, their efficacy reduces over time as the MOs are diluted as the zebrafish embryo develops. The knockdown efficiency is effective in the first 5 days post fertilization [111, 112], which means they can be used during the entire period of zebrafish embryonic development but not beyond.

Morpholinos only cause a knockdown of the gene of investigation. To test the specific effect of a mutation, alternative approaches need to be considered. This can be transient by the injection of zebrafish or human RNA carrying the mutation of interest, or constituent by TALENs or CRISPR/Cas9 mutagenesis. mRNA-based overexpression is a fast and valuable tool for functional analysis of gain-of-function variants for a gene of interest. Also, mRNA injection is often used to verify a loss-of-function phenotype by rescue of gene-knockdown or-knockout phenotypes. As with morpholinos, the injected mRNA is only stable during early stages which is limited to characterize early processes of embryogenesis [113]. Overexpression of human mutant SOD1A4V, SOD1G93A or SOD1G37R mRNA resulted in aberrant ventral root projections in zebrafish embryos [114]. Similar results were obtained in mouse models of SOD1 in which motor neuron pathology begins at the distal axon and progresses in a "dying back" pattern. Taken together, these results indicated that early, notable changes occur at the neuromuscular junctions long before the manifestation of the disease, which could also be the case for individuals with ALS. An advantage of the larval zebrafish is that it is particularly amenable to investigate electrophysiology not only at the periphery neuromuscular junctions, but also centrally, within the spinal cord. SOD1 was the first dominant gain-of function model for studying neurodegenerative diseases in zebrafish. Another example concerns epilepsy. The STXBP1 gene is associated childhood epilepsies and other neurodevelopmental disorders. The CRISPR/Cas9-mediated gene correction was employed in zebrafish to investigate the effect of STXBP1 knock-out and the mutant lines recapitulated clinical phenotypes linked to human STXBP1 mutations [115]. The recent advance of CRISPR/Cas9-mediated genome editing hold great promise for replicating specific patient-derived mutations in zebrafish [116]. Given the structural and functional con- 
servation of human epilepsy genes and zebrafish counterpart, editing genes in zebrafish could reveal fundamental insights as well, possibly leading to new treatments.

The transparency of zebrafish embryos makes fusion of fluorescent protein to the disease-causing transgene especially useful for morphological analysis. Fluorescently tagging mitochondrial proteins can be used to monitor for example mitophagy in an intact organism under a variety of conditions. A zebrafish line expressing fluorescently tagged mitochondria specifically in sensory neurons has recently developed [117]. This model allows the researcher to follow mitochondrial flux in single axons using timelapse microscopy. Since the expression of the fluorescent mitochondrial tag is GAL4 dependent, crossing with any appropriate GAL4-driver line enables other tissue- or cell specific expression (see Drosophila section) [118]. The Zebrafish Model Organism Database (ZFIN; http://zfin.org) is the central resource for zebrafish genetic, genomic, phenotypic and developmental data, providing a broad variety of mutant lines for further investigation.

\subsection{DROSOPHILA MODEL}

Drosophila melanogaster is a genetic model organism, already available since 1900 . For some 50 years from 1910 to 1960, the genetic approaches focused on dissecting the principles of inheritance. Important concepts and tools were developed allowing the study of many other biological processes between 1960-2010 such as neurogenesis, neuronal migration and growth cone guidance [119]. The continuous development of tools to manipulate Drosophila has provided extensive information about the fundamental features of nervous system organization and function and how specific genes can cause neurological diseases [119]. While less complicated than the mammalian brain, the Drosophila brain has striking morphological, molecular and functional similarities with the vertebrate brain. Drosophila glia shows substantial overlap in function with each major category of vertebrate glia $[120,121]$ which can be used to gain insights into glial function [122-124].

The first release of the complete genomic sequence of Drosophila was published in 2000. Comparison of the fly genomic sequence with that of humans shows that $75 \%$ of human disease -causing genes have a functional homologue in Drosophila $[125,126]$. Fly nucleotide or protein sequence shows approximately $40 \%$ homologous to human while in conserved functional domains, it can be 80 to $90 \%$ [127]. A combination of well-established genetic and molecular methods and detailed descriptions of their anatomy and development makes Drosophila an ideal animal model to study neuroscience. Drosophila is easy and inexpensive to maintain, breed in the laboratory in large numbers and has a short life cycle of $\sim 10$ days at $25^{\circ} \mathrm{C}$. More importantly, tools to manipulate and control Drosophila genetics are being developed continuously, including lines containing single-gene disruptions, RNAi-mediated tissue specific knockdown, and 
transgenes to manipulate neuronal activity $[128,129]$. Gene manipulation in Drosophila is relatively simple and fast. An experiment with fruit flies as a model can have results in three weeks whereas a comparable experiment in a mouse could take a year.

For example, the GAL4-UAS system [130] is an elegant system, which enables gene specific knockdown in virtually any tissue or cell type. In this system, a line of flies expressing GAL4 in tissue-specific patterns is crossed with another line carrying the gene (or RNAi) of interest downstream of a transcription activation sequence (UAS) to which GAL4 binds to activate transcription. In the resulting progeny of this cross, the gene (or RNAi) of interest will only be expressed in cells where GAL4 is expressed. In this way, a tissue or cell specific knockdown/overexpression can be established. This approach is widely used to study gene function and the consequence of human disease genes. For example, mutations in human SURF1, encoding a protein localized in the inner mitochondrial membrane involved in the assembly of cytochrome oxidase (COX), cause Leigh syndrome [131]. RNAi- mediated knockdown lines for SURF1 in Drosophila showed severely altered mitochondria, behavioral and electrophysiological abnormalities including reduced photo-responsiveness, reduced locomotor speed, impaired optomotor response as well as abnormal electroretinograms [132], which largely phenocopies the human disease. The model represents the biochemical hallmark of the human disease and a neurological phenotype, which helps clarifying the pathogenesis and of Leigh syndrome and identifying clues for therapeutic interventions. The versatile genetic toolbox of Drosophila is continuously expanding, which facilitates manipulation of the Drosophila genome. FlyBase (www.flybase.org)-the leading database and web portal of Drosophila genes and genomes-curates published literature on phenotypes, gene expression, genetic and physical interactions, and many other datasets associated Drosophila genetics [133], which provides researchers with high-quality and comprehensive information.

One obvious disadvantage of using Drosophila is the fact this is a non-vertebrate model organism. This model may not be suitable to study pathophysiological mechanisms which are specific to vertebrates. Some brain disorders such as brain infarcts and brain hemorrhage can also not be analyzed in Drosophila because they lack vessels and blood cells are mainly restricted to primitive hemocytes. Additionally, in some cases the phenotypes of the fly line do not fully recapitulate all human disease manifestations. For example, despite overexpression wild type and mutant human $\alpha$-synuclein in Drosophila recapitulated many characteristics of Parkinson disease (PD) including age-dependent selective DA neuron loss, $\alpha$-synuclein accumulation in Lewy bodies, Lewy neurites [134], Drosophila are imperfect models of PD. They do not express $\alpha$ synuclein and fail to exhibit the cardinal clinical features of PD including bradykinesia, rest tremor, rigidity and postural instability [135]. But even though it was not possible to fully mimic the key neuropathologic and clinical features neurological disorders in humans, Drosophila exhibit sufficient key features of the disease to provide novel insights into the pathogenesis. 


\subsection{YEAST MODEL}

The entire nucleotide sequence of the Saccharomyces cerevisiae genome was completed in 1996, making it the first sequenced eukaryotic genome. Although being a simple and unicellular organism, yeast cells still possess strong similarities to human cells, making yeast a pivotal model for human diseases. Around $60 \%$ of the yeast genes show sequence homology to a human orthologue [136], and up to $30 \%$ of the human diseaserelated genes have yeast homologs [137]. Importantly, yeast and humans share fundamental aspects of eukaryotic cell biology, including cell cycle [138], programmed cell death [139, 140], protein folding, quality control and degradation [141] and mitochondrial function [142]. A short generation time of 1.5 to 3 hours allows fast and easy scale up, which is valuable for high- throughput genetic and small molecule screens [143]. Furthermore, the availability of a diverse and well-adapted toolbox facilitates the introduction of thousands of mutations in practically every gene of the genome and their effect on specific phenotypes can be assessed.

Yeast has been widely used to study mitochondrial function and dysfunction at the level of one-cellular organism, because:

- S. cerevisiae is a facultative anaerobe devoid of mitochondrial alternative oxidase; this means that any mutation affecting OXPHOS components are not lethal which can be maintained in a strict aerobe and analyzed on fermentable medium (usually glucose) [144].

- The OXPHOS system of yeast is highly similar to the human system except that complex I is absent and replaced by a nuclear-encoded NADH dehydrogenase [145].

- Yeast is the only organism in which mitochondria can be transformed. Mutations can be created by reverse genetics in vitro, introduced to yeast, and followed by their integration into wild-type mtDNA by homologous DNA recombination [145].

- In contrast to other organisms, yeast is homoplasmic. Thus, the phenotype is independent of any threshold effect, providing strong evidence for presumed pathological mutations in humans [145].

Yeast has helped to resolve the most common cause of Leigh syndrome associated with COX deficiency [146]. One candidate gene, SURF1 is the human homolog of yeast SHY1 which encodes a mitochondrial protein required for complex IV expression and respiration [147]. Numerous Leigh patients with COX deficiency were subsequently shown to carry SURF1 mutations. Defect in inner mitochondrial membrane fusion have been implicated in the autosomal dominant optic atrophy type 1 (OPA1) that features exhibit progressive loss of vision and degeneration of the optic nerve and retinal ganglion cells [148]. Sequencing the chromosomal region to which OPA1 causing mutations map revealed homology to a yeast gene encoding a dynamin related protein necessary for mtDNA maintenance [149]. The structure of OPA1 gene was constructed on the basis of this homology and OPA1 mutations have been identified that segregated with the dis- 
ease, demonstrating a role for mitochondria in retinal ganglion cell pathophysiology [149]. Yeast is also a good model for disorders resulting from mutations in the mtDNA as in the case of MELAS which is caused by mutations in the human mt-tRNA(Leu)(UUR) based on the high similarity between the human and yeast mt-tRNA(Leu)(UUR) [150]. The yeast cells carrying the homologous mutation were not able to grow on respiratory substrate and a rapid loss of mtDNA, a typical trait of severe defects in mitochondrial protein synthesis, showing a correlation between the severity of the phenotypes of human and yeast tRNA(Leu)(UUR) mutations in MELAS [150].

Although yeast has played an important role to understand human genes with pathological mitochondrial mutations, there are several limitations to the utilization of this organism as a model system of mitochondrial disease. Not all human mt-tRNAs share strong homology with yeast mt-tRNAs which means that not all tRNA mutations can be analyzed. Also, yeast is inherently homoplasmic, which does not allow to study the threshold effect $[151,152]$. Finally, yeast $S$. cerevisiae lacks complex I and needs to be replaced by yeast Yarrowia lipolytica having a vital proton-pumping NADH: ubiquinone oxidoreductase, for structural and functional analyses of mitochondrial complex I [153]. Being a unicellular organism, yeast is not used for studying tissue-specific like neurons, nor are they functionally linked to other cells. Yeast lack neuron-specific morphological structures, such as dendrites, axons and synapses. As a result, the underlying neuron-specific molecular mechanisms cannot be studied [154].

\subsection{IPSC MODEL}

Despite the use of animal models in research has been indispensable, they often do not fully recapitulate all the characteristics of human systems and pathology which as indicated might also differ among humans themselves. As a result, many drug candidates that pass animal tests, failed in clinical trials in humans. For example, one of the most devastating failures in translational stroke research, NXY-059, failed after two large phase 3 randomised clinical trials despite substantial reported benefit in a range of animal studies [155]. Also, in 2007, a drug to treat Parkinson's disease known as CEP1347 was ineffective in clinical trial in humans after favorable outcomes in animal models [156]. Further examples include Cogane, a trial drug which demonstrated neuroprotective effects against Parkinson's disease in animals but failed in human trials in 2013 [157]. A recent US study concluded that only one of every eight drugs entering clinical trials, will be approved, with $80 \%$ of new drugs being abandoned by drugs companies [158], again indicating that there is a limitation in using animal models to determine the efficacy of novel drugs. As an alternatively, human cell lines can be used. However, these cell lines often do not represent the affected tissue and the affected genes/pathways are not always expressed or lead to a phenotype in these cell lines. These problems have been pertly overcome by the development of the induced plu- 
ripotent stem cells (iPSCs) technology, which was first introduced by Takahashi and Yamanaka in 2006 [159] by reprogramming fibroblast cells from mouse and humans to a pluripotent stage [160]. iPSCs provide an unlimited cell source which overcomes the main obstacle of the limited proliferation potential of traditional cell lines [161]. Moreover, iPSCs can be differentiated into virtually any cell type to be used as model development and disease, providing cell replacement therapies.

To date, patient- specific iPSC lines have been established from a broad variety of patients and covering multiple tissue-specific cell lines, as models of cardiovascular diseases [162, 163], chromosomal disorders [164, 165], progeroid syndromes [166, 167], renal disease [168], hematologic diseases [169-171], metabolic diseases [172, 173], psychiatric disorder [174], neurological degenerative diseases [175-177], muscular disorder [178], and mitochondrial diseases [179, 180].

It is important to differentiate the iPSCs to the exact cell type, in which the genetic defect manifest, and differentiation has been successful to generate different neuronal cell lines including astrocytes, oligodendroglia and other neural stem cells from a heterogeneous population of iPSC-derived neural cells. For example, iPSCs have been successfully differentiated into motor neurons, which have been used as models for ALS studies $[175,181]$. Moreover, this methodology has been applied to study several other neurological disorders including Alzheimer's disease (AD) [182-184], Parkinson's disease (PD) [185] and Huntington disease [186]. Of these, neuronal cultures derived from spinal muscular atrophy (SMA)-iPSC lines exhibited symptom-relevant cell types such as a reduced capacity to form motor neurons and an abnormality in neurite outgrowth, resembling SMA patients [176]. Peripheral neurons from human iPSCs of patients suffering from familial dysautonomia (FD) revealed defects in neurogenic differentiation and migration of neuronal precursors which is compatible with the pathological observations in FD patients [187].

iPSCs are not only promising for therapeutic approaches but also for personalized medicine [188]. They are genetically identical to the donor, preventing the occurrence of an immune rejection in autologous transplantations, allowing the development of patient-specific cell therapy [189]. In addition, patients with the same genetic defect might have different clinical manifestations and pathology, and patient- specific iPSCs do not only contain the mutated gene, but also all the genetic modifiers that possibly play a role in the pathophysiological mechanism. This will enable the identification of modifiers, but also personalized treatment trials on these iPSC-derived cells. Unlike embryonic stem cells, iPSCs do not require use of human oocytes or embryos and, therefore, they are not associated with bioethical problems.

Although iPSC technology for disease modeling and research is progressing rapidly, several issues still remain to be resolved [190]. One challenge in generating and manipulating iPSCs is to produce the required differentiated and functional cell types and tissues. Currently, the differentiation protocols try to mimic embryonic specification and patterning. This approach, however, generally produces a heterogeneous cell popula- 
tion, presenting a possible challenge to phenotype identification linked to specific cell types [191]. Gene expression and biochemical analyses cannot be undertaken without proper normalization for the cell type and their proportion present in these mixed population. Recently, new techniques have been developed to produce reporter cells lines in which genes are fluorescently tagged $[192,193]$. This allows the identification and/or isolation of specific cell types. Moreover, insertion of multiple fluorescent reporters in the same line enables monitoring cell differentiation, maturation and function in real time. Several studies reported the application of genome editing technologies to insert fluorescent proteins into loci in the undifferentiated state of hiPSCs [194]. For example, to overcome the difficulties of cell type heterogeneity during the differentiation process from iPSCs to astroglial cells (iPS astrocytes), ZFN-mediated methods have been employed to generate GFAP-GFP astrocyte reporter lines from two ALS patient hiPSC lines. Subsequent analysis of engraftment of GFP-iPS astrocytes into rat spinal cord grey matter demonstrate their survival and functional maturity, thereby paving the way for future cell-based therapies to treat ALS [195]. A similar approach was employed to generate a fluorescent reporter line to mark MYF5+ cells, an early myogenic specification gene, to allow prospective identification and purification of myogenic progenitors from human iPSCs [196].

A disadvantage related to the cell culture system is the inability of the iPSCs model to replicate the pathophysiological processes at the tissue or system levels. For example, the iPS platform may not replicate a defined anatomical localization and cell-cell interactions in Parkinson disease, although iPSC derived subtypes of neurons (e.g. dopaminergic neurons in Parkinson's disease) allows the study of molecular pathways prior to or during development of disease onset. Sebler et al. [197] described the study of iPSCs derived from three Parkinson disease patients carrying mutations in PINK1, a gene encoding a mitochondrial kinase implicated in the regulation of mitochondrial degradation. These iPSCs were differentiated into dopaminergic neurons. The study reported that dopaminergic neurons from the Parkinson disease patients had decreased mitochondrial recruitment of lentivirally expressed Parkin, increased mitochondrial copy number, and upregulation of PGC-1 $\alpha$, a key regulator of mitochondrial biogenesis. This phenotype was rescued by lentiviral expression of wild-type PINK1 [197].

The recent availability of stem cell derived organoid by 3D cultures provides a new generation of biological models, which mimics the heterogeneous cell types present in the organ, the processes of self-organization of the tissue, and physiological functions generated by tissue-specific cells [198]. 3D culture has advantages for modeling neurological disorders by providing a local environment comparable to in vivo environments and by accelerating neuronal differentiation and neural network formation [199-204]. Besides morphology, neurons grown in a 3D environment are able to express genes such as neuronal markers more accurately, as opposed to 2D monolayer culture [205]. For instance, 3D culture conditions elevated 4-repeat adult tau (4R tau) isoforms, which was essential for recapitulating tau pathology in the model of $A D$ [206]. Although there 
are many potential uses for organoids, current technology has still limitations. The spontaneous nature of their self- organization results in variability in morphology between various areas of the tissue [207, 208] and between different batches [209], making it difficult to perform high-throughput screening with a robust and reliable readout. In time, organoids will bridge the gap between conventional cell culture and in vivo physiology resulting in more detailed knowledge on pathophysiological mechanisms which is essential for the development of therapeutic strategies.

\subsection{ONE MODEL DOESN'T FIT ALL}

Many basic biological, physiological and neurological properties are well conserved from yeast to humans. Yeasts have a short life span, are easy to manipulate genetically which allows to study many of the basic cellular mechanisms at a single cell level involved in neurological diseases, such as mitochondrial defects, protein misfolding, and protein trafficking/degradation impairments [210]. In order of increasing complexity, Drosophila have been used as a model system to describe the disease phenotypes and progression at the cellular and molecular levels [211]. These models provide the insights into many genetic pathways that subsequently have been implicated in human disease. In addition to the genetic approaches, Drosophila can be used to screen chemical compounds, which can be easily achieved by raising flies with drug-treated food to which a defined concentration of the compound has been added [212]. However, being invertebrate, they lack organ systems and many structures that are involved in human disease pathogenesis, and their role in modelling human disease will therefore be limited. Zebrafish as a vertebrate model system has many advantages for understanding molecular mechanisms in a physiological and/or pathophysiological context [213, 214]. Zebrafish have been particularly useful in investigating developmental processes because of their large transparent embryos that develop outside the mother. In addition, zebrafish biology allows high-throughput screening for small molecule chemical compounds of disease pathogenesis. Reverse genetic approaches using knockouts and transgenes are a potentially important tool for studying human genes by first testing their function in zebrafish. Despite these advantages, zebrafish are not mammals and they have numerous genes duplicated [215]. Mice are genetically and physiologically most close to humans but experiments with mice are labor intensive, costly and not particularly well suited for high-throughput screening [216]. Although animal models have been an important source of in vivo studies, they are limited in their ability to mimic the extremely complex process of human physiology and progression. iPSCs allow studying disease mechanisms in any cell type and with the patient's own unique genetic constellation. The potential of iPSCs to differentiate into unlimited source of any cell types would enable the investigation of disease mechanisms in diseases on a large scale without the need for invasive surgical techniques $[217,218]$. However, iPSCs are heter- 
ogeneous both with respect to cell population and differentiation potential. Therefore, iPSCs need to be carefully selected and thoroughly characterized for their pluripotency before clinical application. iPS platform may not be suitable to study cell-cell interactions in a cellular context that mimics human physiology and development.

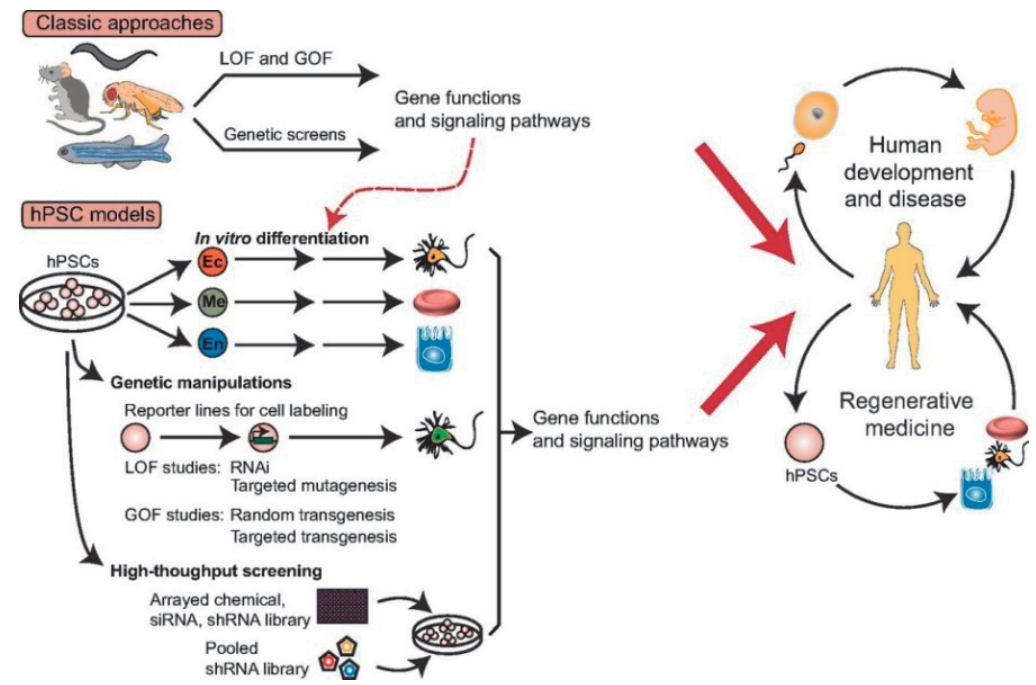

Fig 1. A schematic diagram illustrating animal models and the generation of disease-specific induced pluripotent stem cells (iPSCs) and their applications in modeling disease phenotypes [219].

Neurological diseases represent an enormous burden for society. Significant advancements have been made to determine the genetic causes of many neurological diseases through the utilization of NGS technology. However, the inaccessibility of the relevant tissues and cell types in the central nervous system (CNS) and the complex multifactorial nature of most neurological disorders have hampered research progress and therapy development. Several model systems are currently being utilized to provide such evidence in functional analyses. While every system exhibits advantages and disadvantages which does not sufficiently reflect disease in humans, a combination of models will make it possible to gain insights into pathogenic mechanisms of neurological diseases and reveal novel targets for therapeutic interventions. 


\section{REFERENCES}

1. Boycott, K.M., et al., Rare-disease genetics in the era of next-generation sequencing: discovery to translation. Nat Rev Genet, 2013. 14(10): p. 681-91.

2. McKusick, V.A., Mendelian Inheritance in Man and its online version, OMIM. Am J Hum Genet, 2007. 80(4): p. 588-604.

3. Federico, A., Rare neurological diseases: a Pandora's box for neurology (an European and Italian perspective). Rev Neurol (Paris), 2013. 169 Suppl 1: p. S12-7.

4. Gerards, M., S.C. Sallevelt, and H.J. Smeets, Leigh syndrome: Resolving the clinical and genetic heterogeneity paves the way for treatment options. Mol Genet Metab, 2016. 117(3): p. 300-12.

5. Chinnery, P.F., Mitochondrial Disorders Overview, in GeneReviews(R), R.A. Pagon, et al., Editors. 1993: Seattle (WA).

6. Xue, Y., et al., Solving the molecular diagnostic testing conundrum for Mendelian disorders in the era of next-generation sequencing: single-gene, gene panel, or exome/genome sequencing. Genet Med, 2015. 17(6): p. 444-51.

7. Ankala, A., et al., A comprehensive genomic approach for neuromuscular diseases gives a high diagnostic yield. Ann Neurol, 2015. 77(2): p. 206-14.

8. Wang, W., et al., Target-enrichment sequencing and copy number evaluation in inherited polyneuropathy. Neurology, 2016. 86(19): p. 1762-71.

9. Nemeth, A.H., et al., Next generation sequencing for molecular diagnosis of neurological disorders using ataxias as a model. Brain, 2013. 136(Pt 10): p. 3106-18.

10. Platt, J., R. Cox, and G.M. Enns, Points to consider in the clinical use of NGS panels for mitochondrial disease: an analysis of gene inclusion and consent forms. J Genet Couns, 2014. 23(4): p. 594-603.

11. Niedzicka, M., et al., Molecular Inversion Probes for targeted resequencing in non-model organisms. Sci Rep, 2016. 6: p. 24051.

12. Newman, W.G. and G.C. Black, Delivery of a clinical genomics service. Genes (Basel), 2014. 5(4): p. 100117.

13. Klein, C.J., et al., Application of whole exome sequencing in undiagnosed inherited polyneuropathies. J Neurol Neurosurg Psychiatry, 2014. 85(11): p. 1265-72.

14. Wang, J., et al., Diagnostic yield of clinical next-generation sequencing panels for epilepsy. JAMA Neurol, 2014. 71(5): p. 650-1.

15. Rossor, A.M., M.R. Evans, and M.M. Reilly, A practical approach to the genetic neuropathies. Pract Neurol, 2015. 15(3): p. 187-98.

16. Kumar, K.R., et al., Targeted next generation sequencing in SPAST-negative hereditary spastic paraplegia. J Neurol, 2013. 260(10): p. 2516-22.

17. Lieber, D.S., et al., Targeted exome sequencing of suspected mitochondrial disorders. Neurology, 2013. 80(19): p. 1762-70.

18. Calvo, S.E., et al., Molecular diagnosis of infantile mitochondrial disease with targeted next-generation sequencing. Sci Transl Med, 2012. 4(118): p. 118ra10.

19. DaRe, J.T., et al., Targeted exome sequencing for mitochondrial disorders reveals high genetic heterogeneity. BMC Med Genet, 2013. 14: p. 118.

20. Wortmann, S.B., et al., Whole exome sequencing of suspected mitochondrial patients in clinical practice. J Inherit Metab Dis, 2015. 38(3): p. 437-43.

21. Pronicka, E., et al., New perspective in diagnostics of mitochondrial disorders: two years' experience with whole-exome sequencing at a national paediatric centre. J Transl Med, 2016. 14(1): p. 174.

22. Choi, M., et al., Genetic diagnosis by whole exome capture and massively parallel DNA sequencing. Proc Natl Acad Sci U S A, 2009. 106(45): p. 19096-101.

23. Picardi, E. and G. Pesole, Mitochondrial genomes gleaned from human whole-exome sequencing. Nat Methods, 2012. 9(6): p. 523-4. 
24. Castle, J.C., et al., DNA copy number, including telomeres and mitochondria, assayed using nextgeneration sequencing. BMC Genomics, 2010. 11: p. 244.

25. Larman, T.C., et al., Spectrum of somatic mitochondrial mutations in five cancers. Proc Natl Acad Sci U S A, 2012. 109(35): p. 14087-91.

26. Samuels, D.C., et al., Finding the lost treasures in exome sequencing data. Trends Genet, 2013. 29(10): p. 593-9.

27. van Dijk, E.L., Y. Jaszczyszyn, and C. Thermes, Library preparation methods for next-generation sequencing: tone down the bias. Exp Cell Res, 2014. 322(1): p. 12-20.

28. Green, B., et al., Insertion site preference of Mu, Tn5, and Tn7 transposons. Mob DNA, 2012. 3(1): p. 3.

29. Lelieveld, S.H., et al., Comparison of Exome and Genome Sequencing Technologies for the Complete Capture of Protein-Coding Regions. Hum Mutat, 2015. 36(8): p. 815-22.

30. Aird, D., et al., Analyzing and minimizing PCR amplification bias in Illumina sequencing libraries. Genome Biol, 2011. 12(2): p. R18.

31. Yang, Y., et al., Clinical whole-exome sequencing for the diagnosis of mendelian disorders. N Engl J Med, 2013. 369(16): p. 1502-11.

32. Yang, Y., et al., Molecular findings among patients referred for clinical whole-exome sequencing. JAMA, 2014. 312(18): p. 1870-9.

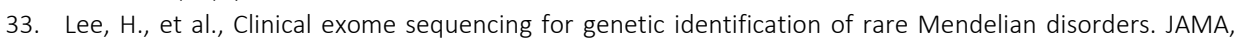
2014. 312(18): p. 1880-7.

34. Database, H.G.M.

35. Lee, J.A. and J.R. Lupski, Genomic rearrangements and gene copy-number alterations as a cause of nervous system disorders. Neuron, 2006. 52(1): p. 103-21.

36. Redon, R., et al., Global variation in copy number in the human genome. Nature, 2006. 444(7118): $p$. 444-54.

37. Lee, J.A., C.M. Carvalho, and J.R. Lupski, A DNA replication mechanism for generating nonrecurrent rearrangements associated with genomic disorders. Cell, 2007. 131(7): p. 1235-47.

38. Pang, A.W., et al., Performance of high-throughput sequencing for the discovery of genetic variation across the complete size spectrum. G3 (Bethesda), 2014. 4(1): p. 63-5.

39. Lupski, J.R., et al., Whole-genome sequencing in a patient with Charcot-Marie-Tooth neuropathy. N Engl J Med, 2010. 362(13): p. 1181-91.

40. Herdewyn, S., et al., Whole-genome sequencing reveals a coding non-pathogenic variant tagging a noncoding pathogenic hexanucleotide repeat expansion in C9orf72 as cause of amyotrophic lateral sclerosis. Hum Mol Genet, 2012. 21(11): p. 2412-9.

41. Bae, B.I., et al., Evolutionarily dynamic alternative splicing of GPR56 regulates regional cerebral cortical patterning. Science, 2014. 343(6172): p. 764-8.

42. Weedon, M.N., et al., Recessive mutations in a distal PTF1A enhancer cause isolated pancreatic agenesis. Nat Genet, 2014. 46(1): p. 61-64.

43. Martin, H.C., et al., Clinical whole-genome sequencing in severe early-onset epilepsy reveals new genes and improves molecular diagnosis. Hum Mol Genet, 2014. 23(12): p. 3200-11.

44. Gonzaga-Jauregui, C., et al., Mutations in VRK1 associated with complex motor and sensory axonal neuropathy plus microcephaly. JAMA Neurol, 2013. 70(12): p. 1491-8.

45. Gilissen, C., et al., Genome sequencing identifies major causes of severe intellectual disability. Nature, 2014. 511(7509): p. 344-7.

46. Available from: http://www.sciencemag.org/news/2016/02/nih-s-1-million-volunteer-precisionmedicine-study-announces-first-pilot-projects.

47. Wang, Z., M. Gerstein, and M. Snyder, RNA-Seq: a revolutionary tool for transcriptomics. Nat Rev Genet, 2009. 10(1): p. 57-63.

48. Morozova, O., M. Hirst, and M.A. Marra, Applications of new sequencing technologies for transcriptome analysis. Annu Rev Genomics Hum Genet, 2009. 10: p. 135-51.

49. Kremer, L.S., et al., Genetic diagnosis of Mendelian disorders via RNA sequencing. Nat Commun, 2017. 8: p. 15824. 
50. Cummings, B.B., et al., Improving genetic diagnosis in Mendelian disease with transcriptome sequencing. Sci Transl Med, 2017. 9(386).

51. Cordero, P. and E.A. Ashley, Whole-genome sequencing in personalized therapeutics. Clin Pharmacol Ther, 2012. 91(6): p. 1001-9.

52. Lo, Y.M., et al., Presence of fetal DNA in maternal plasma and serum. Lancet, 1997. 350(9076): p. 485-7.

53. Fan, H.C., et al., Non-invasive prenatal measurement of the fetal genome. Nature, 2012. 487(7407): p. 320-4.

54. Kitzman, J.O., et al., Noninvasive whole-genome sequencing of a human fetus. Sci Transl Med, 2012. 4(137): p. $137 \mathrm{ra} 76$.

55. Gerards, M., et al., Exome sequencing reveals a novel Moroccan founder mutation in SLC19A3 as a new cause of early-childhood fatal Leigh syndrome. Brain, 2013. 136(Pt 3): p. 882-90.

56. Gerards, M., et al., Riboflavin-responsive oxidative phosphorylation complex I deficiency caused by defective ACAD9: new function for an old gene. Brain, 2011. 134(Pt 1): p. 210-9.

57. Haack, T.B., et al., Exome sequencing identifies ACAD9 mutations as a cause of complex I deficiency. Nat Genet, 2010. 42(12): p. 1131-4.

58. Nouws, J., et al., Acyl-CoA dehydrogenase 9 is required for the biogenesis of oxidative phosphorylation complex I. Cell Metab, 2010. 12(3): p. 283-94.

59. Bonakdar, R.A. and E. Guarneri, Coenzyme Q10. Am Fam Physician, 2005. 72(6): p. 1065-70.

60. Scarpelli, M., et al., Strategies for treating mitochondrial disorders: an update. Mol Genet Metab, 2014. 113(4): p. 253-60.

61. Dixon-Salazar, T.J., et al., Exome sequencing can improve diagnosis and alter patient management. Sci Transl Med, 2012. 4(138): p. 138ra78.

62. Mynarek, M., et al., Allogeneic hematopoietic SCT for alpha-mannosidosis: an analysis of 17 patients. Bone Marrow Transplant, 2012. 47(3): p. 352-9.

63. van Karnebeek, C.D., et al., The metabolic evaluation of the child with an intellectual developmental disorder: diagnostic algorithm for identification of treatable causes and new digital resource. Mol Genet Metab, 2014. 111(4): p. 428-38.

64. Need, A.C., et al., Clinical application of exome sequencing in undiagnosed genetic conditions. J Med Genet, 2012. 49(6): p. 353-61.

65. Janus, C. and D. Westaway, Transgenic mouse models of Alzheimer's disease. Physiol Behav, 2001. 73(5): p. 873-86.

66. Menalled, L.B. and M.F. Chesselet, Mouse models of Huntington's disease. Trends Pharmacol Sci, 2002. 23(1): p. 32-9.

67. Takahashi, K., et al., Induction of pluripotent stem cells from fibroblast cultures. Nat Protoc, 2007. 2(12): p. 3081-9.

68. Benuskova, L. and N. Kasabov, Modeling brain dynamics using computational neurogenetic approach. Cogn Neurodyn, 2008. 2(4): p. 319-34.

69. Rubin, J.E., et al., Basal ganglia activity patterns in parkinsonism and computational modeling of their downstream effects. Eur J Neurosci, 2012. 36(2): p. 2213-28.

70. In Vitro Mutagenesis. Methods and Protocols. Anticancer Res, 2017. 37(3): p. 1542.

71. Subbotina, E., et al., Morpholino-driven gene editing: A new horizon for disease treatment and prevention. Clin Pharmacol Ther, 2016. 99(1): p. 21-5.

72. Wienholds, E., et al., Efficient target-selected mutagenesis in zebrafish. Genome Res, 2003. 13(12): p. 2700-7.

73. Doyon, Y., et al., Heritable targeted gene disruption in zebrafish using designed zinc-finger nucleases. Nat Biotechnol, 2008. 26(6): p. 702-8.

74. Huang, P., et al., Heritable gene targeting in zebrafish using customized TALENs. Nat Biotechnol, 2011. 29(8): p. 699-700.

75. Hwang, W.Y., et al., Efficient genome editing in zebrafish using a CRISPR-Cas system. Nat Biotechnol, 2013. 31(3): p. 227-9. 
76. Smits, P., J. Smeitink, and L. van den Heuvel, Mitochondrial translation and beyond: processes implicated in combined oxidative phosphorylation deficiencies. J Biomed Biotechnol, 2010. 2010: p. 737385.

77. Carroll, C.J., et al., Whole-exome sequencing identifies a mutation in the mitochondrial ribosome protein MRPL44 to underlie mitochondrial infantile cardiomyopathy. J Med Genet, 2013. 50(3): p. 151-9.

78. Emdadul Haque, M., et al., The effect of mutated mitochondrial ribosomal proteins S16 and S22 on the assembly of the small and large ribosomal subunits in human mitochondria. Mitochondrion, 2008. 8(3): p. 254-61.

79. Schlame, M., Cardiolipin remodeling and the function of tafazzin. Biochim Biophys Acta, 2013. 1831(3): p. 582-8.

80. Wortmann, S.B., et al., Mutations in the phospholipid remodeling gene SERAC1 impair mitochondrial function and intracellular cholesterol trafficking and cause dystonia and deafness. Nat Genet, 2012. 44(7): p. 797-802.

81. Mayr, J.A., et al., Lack of the mitochondrial protein acylglycerol kinase causes Sengers syndrome. Am J Hum Genet, 2012. 90(2): p. 314-20.

82. Dutta, S. and P. Sengupta, Men and mice: Relating their ages. Life Sci, 2016. 152: p. 244-8.

83. Phifer-Rixey, M. and M.W. Nachman, Insights into mammalian biology from the wild house mouse Mus musculus. Elife, 2015. 4.

84. Uhl, E.W. and N.J. Warner, Mouse Models as Predictors of Human Responses: Evolutionary Medicine. Curr Pathobiol Rep, 2015. 3(3): p. 219-223.

85. Beck, J.A., et al., Genealogies of mouse inbred strains. Nat Genet, 2000. 24(1): p. 23-5.

86. Hrabe de Angelis, M.H., et al., Genome-wide, large-scale production of mutant mice by ENU mutagenesis. Nat Genet, 2000. 25(4): p. 444-7.

87. Saccon, R.A., et al., Is SOD1 loss of function involved in amyotrophic lateral sclerosis? Brain, 2013. 136(Pt 8): p. 2342-58.

88. Nicholson, S.J., et al., Mice, the motor system, and human motor neuron pathology. Mamm Genome, 2000. 11(12): p. 1041-52.

89. Anderson, S.L., et al., A novel mutation in NDUFS4 causes Leigh syndrome in an Ashkenazi Jewish family. J Inherit Metab Dis, 2008. 31 Suppl 2: p. S461-7.

90. Kruse, S.E., et al., Mice with mitochondrial complex I deficiency develop a fatal encephalomyopathy. Cell Metab, 2008. 7(4): p. 312-20.

91. Quintana, A., et al., Complex I deficiency due to loss of Ndufs4 in the brain results in progressive encephalopathy resembling Leigh syndrome. Proc Natl Acad Sci U S A, 2010. 107(24): p. 10996-1001.

92. Cohen-Tannoudji, M., et al., Disruption of murine Hexa gene leads to enzymatic deficiency and to neuronal lysosomal storage, similar to that observed in Tay-Sachs disease. Mamm Genome, 1995. 6(12): p. 844-9.

93. Phaneuf, D., et al., Dramatically different phenotypes in mouse models of human Tay-Sachs and Sandhoff diseases. Hum Mol Genet, 1996. 5(1): p. 1-14.

94. Sango, K., et al., Mouse models of Tay-Sachs and Sandhoff diseases differ in neurologic phenotype and ganglioside metabolism. Nat Genet, 1995. 11(2): p. 170-6.

95. Lebovitz, R.M., et al., Neurodegeneration, myocardial injury, and perinatal death in mitochondrial superoxide dismutase-deficient mice. Proc Natl Acad Sci U S A, 1996. 93(18): p. 9782-7.

96. Agostino, A., et al., Constitutive knockout of Surf1 is associated with high embryonic lethality, mitochondrial disease and cytochrome c oxidase deficiency in mice. Hum Mol Genet, 2003. 12(4): p. 399-413.

97. Howe, K., et al., The zebrafish reference genome sequence and its relationship to the human genome. Nature, 2013. 496(7446): p. 498-503.

98. Force, A., et al., Preservation of duplicate genes by complementary, degenerative mutations. Genetics, 1999. 151(4): p. 1531-45.

99. Spitsbergen, J.M. and M.L. Kent, The state of the art of the zebrafish model for toxicology and toxicologic pathology research--advantages and current limitations. Toxicol Pathol, 2003. 31 Suppl: p. 62-87.

100. Callaerts, P., et al., Isolation and expression of a Pax-6 gene in the regenerating and intact Planarian Dugesia(G)tigrina. Proc Natl Acad Sci U S A, 1999. 96(2): p. 558-63. 
101. van Heyningen, V. and K.A. Williamson, PAX6 in sensory development. Hum Mol Genet, 2002. 11(10): p. 1161-7.

102. Glaser, T., et al., PAX6 gene dosage effect in a family with congenital cataracts, aniridia, anophthalmia and central nervous system defects. Nat Genet, 1994. 7(4): p. 463-71.

103. Solomon, B.D., et al., Compound heterozygosity for mutations in PAX6 in a patient with complex brain anomaly, neonatal diabetes mellitus, and microophthalmia. Am J Med Genet A, 2009. 149A(11): p. 25436.

104. Kleinjan, D.A., et al., Subfunctionalization of duplicated zebrafish pax6 genes by cis-regulatory divergence. PLoS Genet, 2008. 4(2): p. e29.

105. Nasevicius, A. and S.C. Ekker, Effective targeted gene 'knockdown' in zebrafish. Nat Genet, 2000. 26(2): p. 216-20.

106. Jessen, J.R., et al., Modification of bacterial artificial chromosomes through chi-stimulated homologous recombination and its application in zebrafish transgenesis. Proc Natl Acad Sci U S A, 1998. 95(9): p. 5121-6.

107. Holder, N. and Q. Xu, Microinjection of DNA, RNA, and protein into the fertilized zebrafish egg for analysis of gene function. Methods Mol Biol, 1999. 97: p. 487-90.

108. Moulton, J.D. and Y.L. Yan, Using Morpholinos to control gene expression. Curr Protoc Mol Biol, 2008. Chapter 26: p. Unit 268.

109. Eisen, J.S. and J.C. Smith, Controlling morpholino experiments: don't stop making antisense. Development, 2008. 135(10): p. 1735-43.

110. Robu, M.E., et al., p53 activation by knockdown technologies. PLoS Genet, 2007. 3(5): p. e78.

111. Timme-Laragy, A.R., et al., Antioxidant responses and NRF2 in synergistic developmental toxicity of PAHs in zebrafish. Toxicol Sci, 2009. 109(2): p. 217-27.

112. Bill, B.R., et al., A primer for morpholino use in zebrafish. Zebrafish, 2009. 6(1): p. 69-77.

113. Kabashi, E., et al., Zebrafish models for the functional genomics of neurogenetic disorders. Biochim Biophys Acta, 2011. 1812(3): p. 335-45.

114. Lemmens, R., et al., Overexpression of mutant superoxide dismutase 1 causes a motor axonopathy in the zebrafish. Hum Mol Genet, 2007. 16(19): p. 2359-65.

115. Grone, B.P., et al., Epilepsy, Behavioral Abnormalities, and Physiological Comorbidities in SyntaxinBinding Protein 1 (STXBP1) Mutant Zebrafish. PLoS One, 2016. 11(3): p. e0151148.

116. Irion, U., J. Krauss, and C. Nusslein-Volhard, Precise and efficient genome editing in zebrafish using the CRISPR/Cas9 system. Development, 2014. 141(24): p. 4827-30.

117. Plucinska, G., et al., In vivo imaging of disease-related mitochondrial dynamics in a vertebrate model system. J Neurosci, 2012. 32(46): p. 16203-12.

118. Halpern, M.E., et al., Gal4/UAS transgenic tools and their application to zebrafish. Zebrafish, 2008. 5(2): p. 97-110.

119. Bellen, H.J., C. Tong, and H. Tsuda, 100 years of Drosophila research and its impact on vertebrate neuroscience: a history lesson for the future. Nat Rev Neurosci, 2010. 11(7): p. 514-22.

120. Edwards, T.N. and I.A. Meinertzhagen, The functional organisation of glia in the adult brain of Drosophila and other insects. Prog Neurobiol, 2010. 90(4): p. 471-97.

121. Freeman, M.R. and J. Doherty, Glial cell biology in Drosophila and vertebrates. Trends Neurosci, 2006. 29(2): p. 82-90.

122. Allen, N.J. and B.A. Barres, Neuroscience: Glia - more than just brain glue. Nature, 2009. 457(7230): p. 675-7.

123. Banerjee, S. and M.A. Bhat, Neuron-glial interactions in blood-brain barrier formation. Annu Rev Neurosci, 2007. 30: p. 235-58.

124. Jacobs, J.R. and C.S. Goodman, Embryonic development of axon pathways in the Drosophila CNS. II. Behavior of pioneer growth cones. J Neurosci, 1989. 9(7): p. 2412-22.

125. Reiter, L.T., et al., A systematic analysis of human disease-associated gene sequences in Drosophila melanogaster. Genome Res, 2001. 11(6): p. 1114-25. 
126. Lloyd, T.E. and J.P. Taylor, Flightless flies: Drosophila models of neuromuscular disease. Ann N Y Acad Sci, 2010. 1184: p. e1-20.

127. Rubin, G.M., et al., Comparative genomics of the eukaryotes. Science, 2000. 287(5461): p. 2204-15.

128. Kaun, K.R., A.V. Devineni, and U. Heberlein, Drosophila melanogaster as a model to study drug addiction. Hum Genet, 2012. 131(6): p. 959-75.

129. Venken, K.J., J.H. Simpson, and H.J. Bellen, Genetic manipulation of genes and cells in the nervous system of the fruit fly. Neuron, 2011. 72(2): p. 202-30.

130. Brand, A.H. and N. Perrimon, Targeted gene expression as a means of altering cell fates and generating dominant phenotypes. Development, 1993. 118(2): p. 401-15.

131. Horvath, R., et al., Leigh syndrome caused by mutations in the flavoprotein (Fp) subunit of succinate dehydrogenase (SDHA). J Neurol Neurosurg Psychiatry, 2006. 77(1): p. 74-6.

132. Zordan, M.A., et al., Post-transcriptional silencing and functional characterization of the Drosophila melanogaster homolog of human Surf1. Genetics, 2006. 172(1): p. 229-41.

133. St Pierre, S.E., et al., FlyBase 102--advanced approaches to interrogating FlyBase. Nucleic Acids Res, 2014. 42(Database issue): p. D780-8.

134. Feany, M.B. and W.W. Bender, A Drosophila model of Parkinson's disease. Nature, 2000. 404(6776): p. 394-8.

135. Dawson, T.M., H.S. Ko, and V.L. Dawson, Genetic animal models of Parkinson's disease. Neuron, 2010. 66(5): p. 646-61.

136. Liu, W., et al., From Saccharomyces cerevisiae to human: The important gene co-expression modules. Biomed Rep, 2017. 7(2): p. 153-158.

137. Bassett, D.E., Jr., M.S. Boguski, and P. Hieter, Yeast genes and human disease. Nature, 1996. 379(6566): p. 589-90.

138. Hartwell, L.H., Nobel Lecture. Yeast and cancer. Biosci Rep, 2002. 22(3-4): p. 373-94.

139. Munoz, A.J., et al., Systems biology of yeast cell death. FEMS Yeast Res, 2012. 12(2): p. 249-65.

140. Carmona-Gutierrez, D., et al., Cell death in yeast: growing applications of a dying buddy. Cell Death Differ, 2010. 17(5): p. 733-4.

141. Brodsky, J.L. and W.R. Skach, Protein folding and quality control in the endoplasmic reticulum: Recent lessons from yeast and mammalian cell systems. Curr Opin Cell Biol, 2011. 23(4): p. 464-75.

142. Qian, Y., et al., Yeast cells expressing the human mitochondrial DNA polymerase reveal correlations between polymerase fidelity and human disease progression. J Biol Chem, 2014. 289(9): p. 5970-85.

143. Khurana, V. and S. Lindquist, Modelling neurodegeneration in Saccharomyces cerevisiae: why cook with baker's yeast? Nat Rev Neurosci, 2010. 11(6): p. 436-49.

144. Barrientos, A., Yeast models of human mitochondrial diseases. IUBMB Life, 2003. 55(2): p. 83-95.

145. Lasserre, J.P., et al., Yeast as a system for modeling mitochondrial disease mechanisms and discovering therapies. Dis Model Mech, 2015. 8(6): p. 509-26.

146. Zhu, Z., et al., SURF1, encoding a factor involved in the biogenesis of cytochrome c oxidase, is mutated in Leigh syndrome. Nat Genet, 1998. 20(4): p. 337-43.

147. Barrientos, A., D. Korr, and A. Tzagoloff, Shy1p is necessary for full expression of mitochondrial COX1 in the yeast model of Leigh's syndrome. EMBO J, 2002. 21(1-2): p. 43-52.

148. Votruba, M., A.T. Moore, and S.S. Bhattacharya, Clinical features, molecular genetics, and pathophysiology of dominant optic atrophy. J Med Genet, 1998. 35(10): p. 793-800.

149. Alexander, C., et al., OPA1, encoding a dynamin-related GTPase, is mutated in autosomal dominant optic atrophy linked to chromosome 3q28. Nat Genet, 2000. 26(2): p. 211-5.

150. Feuermann, M., et al., The yeast counterparts of human 'MELAS' mutations cause mitochondrial dysfunction that can be rescued by overexpression of the mitochondrial translation factor EF-Tu. EMBO Rep, 2003. 4(1): p. 53-8.

151. De Luca, C., et al., Mutations in yeast mt tRNAs: specific and general suppression by nuclear encoded tRNA interactors. Gene, 2006. 377: p. 169-76.

152. Montanari, A., et al., Yeast as a model of human mitochondrial tRNA base substitutions: investigation of the molecular basis of respiratory defects. RNA, 2008. 14(2): p. 275-83. 
153. Kerscher, S., et al., Application of the yeast Yarrowia lipolytica as a model to analyse human pathogenic mutations in mitochondrial complex I (NADH:ubiquinone oxidoreductase). Biochim Biophys Acta, 2004. 1659(2-3): p. 197-205.

154. Braun, R.J., et al., Nervous yeast: modeling neurotoxic cell death. Trends Biochem Sci, 2010. 35(3): p. 135-44.

155. Neuroprotection: the end of an era? Lancet, 2006. 368(9547): p. 1548.

156. Parkinson Study Group, P.I., Mixed lineage kinase inhibitor CEP-1347 fails to delay disability in early Parkinson disease. Neurology, 2007. 69(15): p. 1480-90.

157. Stocchi, F., Therapy for Parkinson's disease: what is in the pipeline? Neurotherapeutics, 2014. 11(1): p. 24-33.

158. Avorn J, The \$2.6 billion pill--methodologic and policy considerations. N Engl J Med. 2015. 372(20): p.1877-9.

159. Takahashi, K. and S. Yamanaka, Induction of pluripotent stem cells from mouse embryonic and adult fibroblast cultures by defined factors. Cell, 2006. 126(4): p. 663-76.

160. Takahashi, K., et al., Induction of pluripotent stem cells from adult human fibroblasts by defined factors. Cell, 2007. 131(5): p. 861-72.

161. Chun, Y.S., P. Chaudhari, and Y.Y. Jang, Applications of patient-specific induced pluripotent stem cells; focused on disease modeling, drug screening and therapeutic potentials for liver disease. Int J Biol Sci, 2010. 6(7): p. 796-805.

162. Ma, D., et al., Generation of patient-specific induced pluripotent stem cell-derived cardiomyocytes as a cellular model of arrhythmogenic right ventricular cardiomyopathy. Eur Heart J, 2013. 34(15): p. 112233.

163. Caspi, O., et al., Modeling of arrhythmogenic right ventricular cardiomyopathy with human induced pluripotent stem cells. Circ Cardiovasc Genet, 2013. 6(6): p. 557-68.

164. Shi, Y., et al., A human stem cell model of early Alzheimer's disease pathology in Down syndrome. Sci Transl Med, 2012. 4(124): p. 124ra29.

165. Jiang, J., et al., Translating dosage compensation to trisomy 21. Nature, 2013. 500(7462): p. 296-300.

166. Zhang, J., et al., A human iPSC model of Hutchinson Gilford Progeria reveals vascular smooth muscle and mesenchymal stem cell defects. Cell Stem Cell, 2011. 8(1): p. 31-45.

167. Liu, G.H., et al., Recapitulation of premature ageing with iPSCs from Hutchinson-Gilford progeria syndrome. Nature, 2011. 472(7342): p. 221-5.

168. Freedman, B.S., et al., Reduced ciliary polycystin-2 in induced pluripotent stem cells from polycystic kidney disease patients with PKD1 mutations. J Am Soc Nephrol, 2013. 24(10): p. 1571-86.

169. Raya, A., et al., Disease-corrected haematopoietic progenitors from Fanconi anaemia induced pluripotent stem cells. Nature, 2009. 460(7251): p. 53-9.

170. Pessach, I.M., et al., Induced pluripotent stem cells: a novel frontier in the study of human primary immunodeficiencies. J Allergy Clin Immunol, 2011. 127(6): p. 1400-7 e4.

171. Kumano, K., et al., Generation of induced pluripotent stem cells from primary chronic myelogenous leukemia patient samples. Blood, 2012. 119(26): p. 6234-42.

172. Maehr, R., et al., Generation of pluripotent stem cells from patients with type 1 diabetes. Proc Natl Acad Sci U S A, 2009. 106(37): p. 15768-73.

173. Rashid, S.T., et al., Modeling inherited metabolic disorders of the liver using human induced pluripotent stem cells. J Clin Invest, 2010. 120(9): p. 3127-36.

174. Brennand, K.J., et al., Modelling schizophrenia using human induced pluripotent stem cells. Nature, 2011. 473(7346): p. 221-5.

175. Dimos, J.T., et al., Induced pluripotent stem cells generated from patients with ALS can be differentiated into motor neurons. Science, 2008. 321(5893): p. 1218-21.

176. Ebert, A.D., et al., Induced pluripotent stem cells from a spinal muscular atrophy patient. Nature, 2009. 457(7227): p. 277-80.

177. Lee, G., et al., Modelling pathogenesis and treatment of familial dysautonomia using patient-specific iPSCs. Nature, 2009. 461(7262): p. 402-6. 
178. Luo, Y., et al., Modeling induced pluripotent stem cells from fibroblasts of Duchenne muscular dystrophy patients. Int J Neurosci, 2014. 124(1): p. 12-21.

179. Fujikura, J., et al., Induced pluripotent stem cells generated from diabetic patients with mitochondrial DNA A3243G mutation. Diabetologia, 2012. 55(6): p. 1689-98.

180. Kodaira, M., et al., Impaired respiratory function in MELAS-induced pluripotent stem cells with high heteroplasmy levels. FEBS Open Bio, 2015. 5: p. 219-25.

181. Park, I.H., et al., Disease-specific induced pluripotent stem cells. Cell, 2008. 134(5): p. 877-86.

182. Yagi, T., et al., Modeling familial Alzheimer's disease with induced pluripotent stem cells. Hum Mol Genet, 2011. 20(23): p. 4530-9.

183. Kondo, T., et al., Modeling Alzheimer's disease with iPSCs reveals stress phenotypes associated with intracellular Abeta and differential drug responsiveness. Cell Stem Cell, 2013. 12(4): p. 487-96.

184. Israel, M.A., et al., Probing sporadic and familial Alzheimer's disease using induced pluripotent stem cells. Nature, 2012. 482(7384): p. 216-20.

185. Byers, B., et al., SNCA triplication Parkinson's patient's iPSC-derived DA neurons accumulate alphasynuclein and are susceptible to oxidative stress. PLoS One, 2011. 6(11): p. e26159.

186. Zhang, N., et al., Characterization of Human Huntington's Disease Cell Model from Induced Pluripotent Stem Cells. PLoS Curr, 2010. 2: p. RRN1193.

187. Lee, G. and L. Studer, Modelling familial dysautonomia in human induced pluripotent stem cells. Philos Trans R Soc Lond B Biol Sci, 2011. 366(1575): p. 2286-96.

188. Picanco-Castro, V., et al., Can pluripotent stem cells be used in cell-based therapy? Cell Reprogram, 2014. 16(2): p. 98-107.

189. Liras, A., Future research and therapeutic applications of human stem cells: general, regulatory, and bioethical aspects. J Transl Med, 2010. 8: p. 131

190. Shi, Y., et al., Induced pluripotent stem cell technology: a decade of progress. Nat Rev Drug Discov, 2017. 16(2): p. 115-130.

191. Belinsky, G.S., et al., Patch-clamp recordings and calcium imaging followed by single-cell PCR reveal the developmental profile of 13 genes in iPSC-derived human neurons. Stem Cell Res, 2014. 12(1): p. 101-18.

192. Placantonakis, D.G., et al., BAC transgenesis in human embryonic stem cells as a novel tool to define the human neural lineage. Stem Cells, 2009. 27(3): p. 521-32.

193. Hockemeyer, D., et al., Efficient targeting of expressed and silent genes in human ESCs and iPSCs using zinc-finger nucleases. Nat Biotechnol, 2009. 27(9): p. 851-7.

194. Brookhouser, N., et al., May I Cut in? Gene Editing Approaches in Human Induced Pluripotent Stem Cells. Cells, 2017. 6(1).

195. Zhang, P.W., et al., Generation of GFAP::GFP astrocyte reporter lines from human adult fibroblastderived iPS cells using zinc-finger nuclease technology. Glia, 2016. 64(1): p. 63-75.

196. Wu, J., et al., Generation and Characterization of a MYF5 Reporter Human iPS Cell Line Using CRISPR/Cas9 Mediated Homologous Recombination. Sci Rep, 2016. 6: p. 18759.

197. Seibler, P., et al., Mitochondrial Parkin recruitment is impaired in neurons derived from mutant PINK1 induced pluripotent stem cells. J Neurosci, 2011. 31(16): p. 5970-6.

198. Lancaster, M.A. and J.A. Knoblich, Organogenesis in a dish: modeling development and disease using organoid technologies. Science, 2014. 345(6194): p. 1247125.

199. Liedmann, A., A. Rolfs, and M.J. Frech, Cultivation of human neural progenitor cells in a 3-dimensional self-assembling peptide hydrogel. J Vis Exp, 2012(59): p. e3830.

200. Li, H., A. Wijekoon, and N.D. Leipzig, 3D differentiation of neural stem cells in macroporous photopolymerizable hydrogel scaffolds. PLoS One, 2012. 7(11): p. e48824.

201. Ortinau, S., et al., Effect of 3D-scaffold formation on differentiation and survival in human neural progenitor cells. Biomed Eng Online, 2010. 9: p. 70.

202. Suga, H., et al., Self-formation of functional adenohypophysis in three-dimensional culture. Nature, 2011. 480(7375): p. 57-62.

203. Liedmann, A., et al., Differentiation of human neural progenitor cells in functionalized hydrogel matrices. Biores Open Access, 2012. 1(1): p. 16-24. 
204. Tang-Schomer, M.D., et al., Bioengineered functional brain-like cortical tissue. Proc Natl Acad Sci U S A, 2014. 111(38): p. 13811-6.

205. Seidel, D., et al., Induced tauopathy in a novel 3D-culture model mediates neurodegenerative processes: a real-time study on biochips. PLoS One, 2012. 7(11): p. e49150.

206. D'Avanzo, C., et al., Alzheimer's in 3D culture: challenges and perspectives. Bioessays, 2015. 37(10): p. 1139-48.

207. Eiraku, M., et al., Self-organizing optic-cup morphogenesis in three-dimensional culture. Nature, 2011. 472(7341): p. 51-6.

208. Lancaster, M.A., et al., Cerebral organoids model human brain development and microcephaly. Nature, 2013. 501(7467): p. 373-9.

209. Kelava, I. and M.A. Lancaster, Stem Cell Models of Human Brain Development. Cell Stem Cell, 2016. 18(6): p. 736-48.

210. Clerc, P., S. Lipnick, and C. Willett, A look into the future of ALS research. Drug Discov Today, 2016. 21(6): p. 939-49.

211. Pandey, U.B. and C.D. Nichols, Human disease models in Drosophila melanogaster and the role of the fly in therapeutic drug discovery. Pharmacol Rev, 2011. 63(2): p. 411-36.

212. Hirth, F., Drosophila melanogaster in the study of human neurodegeneration. CNS Neurol Disord Drug Targets, 2010. 9(4): p. 504-23.

213. Bandmann, O. and E.A. Burton, Genetic zebrafish models of neurodegenerative diseases. Neurobiol Dis, 2010. 40(1): p. 58-65.

214. Newman, M., et al., Zebrafish as a tool in Alzheimer's disease research. Biochim Biophys Acta, 2011. 1812(3): p. 346-52.

215. Goldsmith, J.R. and C. Jobin, Think small: zebrafish as a model system of human pathology. J Biomed Biotechnol, 2012. 2012: p. 817341.

216. Hafezparast, M., et al., Mouse models for neurological disease. Lancet Neurol, 2002. 1(4): p. 215-24.

217. Cao, L., et al., Induced Pluripotent Stem Cells for Disease Modeling and Drug Discovery in Neurodegenerative Diseases. Mol Neurobiol, 2015. 52(1): p. 244-55.

218. Haggarty, S.J. and R.H. Perlis, Translation: screening for novel therapeutics with disease-relevant cell types derived from human stem cell models. Biol Psychiatry, 2014. 75(12): p. 952-60.

219. Zengrong Zhu and Danwei Huangfu, Development. Human pluripotent stem cells: an emerging model in developmental biology. 2013 Feb 15; 140(4): 705-717 
Summary 

Neurological disorders primarily affect and impair the functioning of the brain and/or neurological system. Structural, electrical or metabolic abnormalities in the brain or neurological system can result in a range of clinical symptoms, including brain malformation, neuropathies, encephalopathies, myopathies and muscular dystrophies, movement disorders, and dementias. Neurological disorders affect people of all ages with devastating consequences and leading to major morbidity and mortality. Neurological disorders can have many different causes, including genetic defects, infections, trauma, brain injury, but they can also develop as a result of an unhealthy environment, for example malnutrition. The main objective of this thesis is to discover and functionally the genetic cause in genetic neurological disorders, in which mitochondria are expected to play a pathological role. The genetically heterogeneous nature of neurological and mitochondrial diseases makes identifying the disease-causing mutations highly challenging. Mitochondrial diseases are even more complex, as both the mtDNA and the nuclear genome can be involved. We used next-generation sequencing to analyze the mtDNA and the exome in patients with suspected mitochondrial disease as a comprehensive and unbiased approach to identify the genetic cause. Finding the genetic cause is important for diagnosis, prognosis and reproductive options, but understanding the underlying disease mechanisms could reveal novel targets for therapeutic interventions.

Chapter 1 provides an overview of neurological disorders, primarily focusing on those in which defective mitochondria are playing a causative role. These diseases are clinically and genetically heterogeneous, providing considerable limitations to phenotype driven approaches to identify the genetic cause. As mitochondria are under dual genetic control, both the mtDNA and the nuclear genome encode the many hundreds of mitochondrial proteins, each of which can be a candidate in case of mitochondrial diseases. To date, hundreds of nuclear DNA mutations have been identified as cause of mitochondrial diseases and new cases are being resolved on a daily basis, mainly due to the progress in sequencing technology. The impact of next generation sequencing and whole exome or whole genome analysis as effective methods to detect the genetic basis of neurological diseases are being introduced. As these approaches yield huge numbers of variants, accurate filtering strategies and functional models are essential to confirm the role of novel genes and/or variants.

In chapter 2 to 5 , I present the results of whole exome sequencing to identify the genetic defects in 4 families with presumably autosomal recessive, neurological and possibly mitochondrial diseases, in which the mtDNA was excluded. Novel genes and/or novel potentially pathogenic variants were further functionally investigated in patientderived cell lines and in zebrafish models. Chapter 2 reports on a patient from nonconsanguineous Dutch parents, presenting with cerebellar ataxia and atrophy. Exome sequencing revealed that the patient was compound heterozygous for pathogenic variants (c.37G>C; p.D13H and c.946A>T; p.K316*) in the CWF19L1 gene. Variant p.D13H changed the highly conserved, negatively charged aspartic acid into the positively charged histidine and was predicted to affect protein stability and function. The other 
variant led to a premature stop codon p.K316* which resulted in nonsense-mediated mRNA decay. Although the exact function of the C19L1 is still unknown, the nuclear localization in combination with a metallophosphatase domain, which is found in RNA lariat debranching enzymes, suggested a role in mRNA processing. Muscle biopsies showed ragged-red fibers due to accumulation of abnormal mitochondria, which indicated that CWF19L1 could play a role in mitochondrial structure and function. An additional 27 patients with autosomal recessive cerebellar ataxia (ARCA) were tested for pathogenic variants in the CWF19L gene. However, no pathogenic variants were identified in these patients. Our results indicated CWF19L1 mutations as a novel but rare cause of autosomal recessive cerebellar ataxia. Chapter 3 describes WES in a Moroccan girl of consanguineous parents with optic atrophy and cerebellar atrophy. A novel homozygous mutation was detected in the SLC25A46 gene (c.283+3G $>T$ ), which led to alternative splicing, a frameshift and a premature stop codon. SLC25A46 mRNA expression showed there is hardly any wild-type transcript present in the patient. Patients fibroblasts showed a fragmented mitochondrial network, confirming a role for SLC25A46 in mitochondrial dynamics. An additional 10 patients with optic atrophy and cerebellar atrophy, who did not carry mtDNA and OPA1mutations, were tested for pathogenic mutations in the SLC25A46 gene. However, no additional pathogenic variants were identified. Our findings confirmed SLC25A46 as a novel mitochondrial cause for optic atrophy spectrum disorder. In Chapter 4 a patient from non-consanguineous Dutch parents is described, presenting with slowly progressive neurodegeneration, cerebellar ataxia, chorea dystonic movements, polyneuropathy, type 1 diabetes and OXPHOS complex IV deficiency in muscle. Compound heterozygous splice site mutations were identified by WES in the COX18 gene (c.720 G>A and c.828+3 G>C). COX18 catalyzes the proper translocation of the $\mathrm{C}$-terminus of COX2 across the mitochondrial inner membrane, which makes essential for proper COX assembly. Both mutations led to abnormal splicing and a premature stop codon, explaining the severe complex IV deficiency observed in muscle of the patient. Our results indicated COX18 mutations as a novel cause of isolated COX deficiency. Chapter 5 reports on a Turkish patient from a consanguineous family with a severe neurodegenerative condition, including cerebellar atrophy, spasticity, feeding problems and epilepsy. WES combined with homozygosity mapping identified possible pathogenic mutations in 2 candidate genes, HKDC1 and MED20. MED20 mutations have been associated in one case of infantile basal ganglia degeneration and brain atrophy. Our patient presented with more severe neurological symptoms. Therefore, the role of HKDC1 in the phenotype was investigated by performing morpholino (MO) mediated knockdown experiments in zebrafish. Treated zebrafish displayed microcephaly and small eyes, which nicely resembled the human microcephaly phenotype. Our data indicated that defects in HKDC1 as well as MED20 could explain the severe cerebellar atrophy, neuronal degenerative condition, spasticity, feeding problems and epilepsy observed in our patient. It is unclear so far which of them defines the disease manifestation or that it is combined effect of the two. 
In Chapter 6 we described a summary of all gene and gene defects identified in a cohort of 119 patients, mostly children, with either a high suspicion of a mitochondrial disorder or with a disease phenotype in which mitochondrial defects are part of the differential diagnosis. In this cohort, a next generation sequencing based approach was used in two steps. Firstly, the mtDNA was screened for mutations, and secondly, if negative for pathogenic mtDNA mutations, Whole Exome Sequencing (WES) was performed. mtDNA point-mutations and INDELs were detected in $20 \%$ of the patientcohort, and a disease-causing nuclear gene mutation in $50 \%$ of the patients, implying an overall diagnostic yield of $70 \%$. In an additional $7 \%$ of the WES-cohort, a variant with a possible lead to the patient's phenotype was found and further laboratory validation could confirm a role in disease. As 31\% of the disease-causing genes were at the date of genetic diagnosis not present in the MitoCarta database and in some cases more than 1 genetic cause was present, this disqualifies gene panel based approaches and is a plead for a complete analysis of the entire exome, providing a comprehensive overview of all relevant variants. Our results demonstrate that WES is the preferred approach to establish a genetic diagnosis in genetically heterogeneous neurological and/or mitochondrial disease.

The general discussion (Chapter 7) discusses first the importance of next generation sequencing, WES and WGS to identify the genetic cause in neurological diseases with a potential involvement of mitochondria. These developments have resulted in a paradigm shift in the diagnostics of genetically heterogenous disorders, which was phenotype-based and is now largely genotype-based. Impressive progress has been made towards understanding the basis of many neurological diseases. Particular advantages and limitations in NGS are highlighted and the clinical and research applications of these sequencing platforms for neurological disorders are discussed. The advances in finding genetic variants emphasized the need for model systems that give experimental evidence for the role of these variants in disease and characterize the underlying pathophysiological mechanisms. The second part of chapter 7 discusses a variety of model systems, in vitro, in vivo and in silico, which are utilized in neurological disorders. The advantages and limitations of each of these models in studying human neurological diseases based on the extent to which aspects of human phenotypes are recapitulated, are being discussed. 

Valorisation 



\section{SOCIAL AND CLINICAL RELEVANCE}

Until a couple of years ago, a patient with a possible genetic disease was offered genetic testing of known disease genes based on the phenotype. Single gene testing was performed using Sanger sequencing, which was the technical 'gold standard' in clinical genetic testing for the past decade [1]. Candidate genes were mostly sequenced sequentially, which was time-consuming and expensive. As shown in my thesis, advances in new sequencing technologies, referred to as next-generation sequencing (NGS), enabled sequencing of many genes, large candidate regions, the whole exome and even the whole genome in a relative short period of time in an increasingly cost-effective manner. In parallel, rapid developments in bioinformatics have made analysis and interpretation of variants in both known and novel genes more effective and efficient.

The work described in this thesis, shows that neurological patients with likely or possible mitochondrial involvement are particularly suited for NGS sequencing, especially when, which is commonly the case, no evident candidate gene is available. We used a 2step approach beginning with NGS screening for mtDNA defects followed by WES analysis of the unsolved cases. The NGS approach identified an mtDNA defect in $20 \%$ of our patients having characteristic mitochondrial disorders. The sensitivity and specificity were sufficiently high to test blood on patients. In a total of $50 \%$ of the patients a genetic cause was identified using WES, which is a major step forward, compared to conventional Sanger sequencing method, which only solved $11 \%$. Interestingly, $32 \%$ of the disease-causing genes in these patients were at the date of genetic diagnosis not present in the MitoCarta database and therefore would have been missed with targeted sequencing approaches. Based on the success of our work and that of others we can conclude that WES is currently the best approach to unravel the cause in clinically and genetically heterogeneous disease, justifying a rapid introduction into molecular diagnostics, as has happened in the past 2 years. As our work has been performed on a very well-characterized cohort, both with respect to clinical manifestation and more importantly a highly likely genetic cause, it is obvious that in routine patient-care the diagnostic yield will be less. But reassuring is that in case there would be genetic cause the chances of identifying it are high.

Our work shows also that for the group of patients we have been investigating, a targeted gene-panel approach will fall short, as in about $1 / 3$ of the patients, novel genes were involved, not present in the MitoCarta database and/or clinical exome at the date of discovery. WES allows simultaneous analysis of known genes while identifying novel genes linked to the patient phenotype at the same time. In literature, more than 180 novel rare-disease-causing genes have been discovered through WES and more than 130 were reported in 2012 only [2]. We have reported CWF19L1, SLC25A46 as novel disease-causing genes, verified other reports which supported to the candidacy of the genes, and COX18 as a new candidate gene in patients with isolated COX deficiency (chapter $2,3,4$ ). Our work demonstrates that the most optimal diagnostic strategy to 
identify the genetic cause in patients with neurological disorders and a possible mitochondrial involvement is an unbiased, complete exome analysis from the start. Although in diagnostics, mostly a step-wise procedure of a targeted panel followed by an open exome is being performed, this causes considerable delays in achieving a diagnosis for at least $1 / 3$ of the patients.

Another group benefitting from WES analysis are patients with multiple genetic diseases. The presence of two or more separate diseases which may or may not conflate to appear as one adds to the complexity to the diagnosis, as illustrated by the two patients with a broad variety of clinical features in chapter 6 . Three gene defects (ACY1, SERAC1, ANTXR2) in the first patient explained all symptoms, but manifestations were overlapping (blended phenotype). Two gene defects (HPS1, BICD2) in the second patient explained non-overlapping symptoms (composite phenotype) [3]. This is especially an issue in case of consanguinity among the parents. Also, the patient with mutations in HKDC1 and MED2O illustrates that is dangerous to stop when the first genetic defect, which could explain the disease, has been identified with a targeted analysis, as other, equally likely causes might be missed. Based on this data, again, we support an immediate start with WES, being a comprehensive and unbiased approach.

A genetic diagnosis allows a more accurate prognosis, follow-up investigations in families and the possibility to prevent further disease transmission by prenatal diagnosis or preimplantation genetic diagnosis. We have demonstrated that WES is also quick enough, enabling a genetic diagnosis in an affected boy, while his mother was pregnant again. In this case, the mitochondrial encephalomyopathy was caused by FBXL4 mutations, which were tested prenatally for the unborn child [4]. For consanguineous couples in particular, but in future possibly for every couple with a child-wish, WES-based preconception carrier screening (PCS) will be available to detect couples at risk of transmitting recessive genetic disorders they were not aware of. PCS has been applied for centuries in populations with high carrier frequencies for certain diseases and significantly reduced the disease incidence. Currently, carrier screening is becoming available for all individuals, if desired, regardless of ancestry or geographic origin. [5].

While increasing the diagnostic yield, costs of WES are markedly lower than the average total traditional diagnostic costs. The clinical utility of whole-exome sequencing (WES) was investigated for complex pediatric neurology in terms of diagnostic yield and costs [6]. In a parallel study, all patients received both the standard diagnostic workup (e.g., cerebral imaging, muscle biopsies or lumbar punctures, and sequential gene-bygene-based testing) and WES simultaneously. WES yielded significantly more conclusive diagnoses $(29.3 \%)$ than the standard care pathway $(7.3 \%)$ without incurring higher costs. Exploratory analysis of WES as a first-tier diagnostic test indicates that WES may even be cost-saving, depending on the extent of other tests being omitted [6]. This is in line with our studies. For 58 of 118 patients, WES led to a conclusive diagnosis, with no need for additional tests. 
Identification of the genetic defect is not only important for diagnostics, but, unfortunately still in limited cases, also for therapeutic interventions. Patients with SLC25A32, SLC19A3 and TMEM126B defects showed improvement upon treatment with respectively riboflavin, biotin/thiamine and high fat-diet. A successful treatment based on the gene defect which is identified by WES may guide treatment of other patients with the same gene defect. For example, immediate treatment with a high-dose of thiamine and, possibly, biotin for patients with SLC19A3 mutations prevents fatal Leigh syndrome. Despite the significant improvements in the genetic diagnosis of mitochondrial disorders, the development of novel therapies has is lagging behind (chapter 6)

Our study and those of many others demonstrated the diagnostic utility of WES in patients with highly likely genetic, neurological and/or mitochondrial disorders. WES should be offered as the first diagnostic test to those patients. The early use of WES increases the diagnostic yield while reducing the time to diagnosis, the finance and burdens associated with prolonged investigations. 


\section{REFERENCES}

1. Bakker E., Is the DNA sequence the gold standard in genetic testing? Quality of molecular genetic tests assessed. Clin Chem. 2006 Apr; 52(4):557-8.

2. Boycott KM, Vanstone MR, Bulman DE, MacKenzie AE. Rare-disease genetics in the era of nextgeneration sequencing: discovery to translation. Nat Rev Genet. 2013 Oct;14(10):681-91.

3. Theunissen TE, Sallevelt SC, Hellebrekers DM et al., Rapid resolution of blended or composite multigenic disease in infants by whole exome sequencing. J Pediatr. 2017 Mar;182:371-374.e2.

4. Maartje C. van Rij, Fenna A. R. Jansen, Debby M. E. I. Hellebrekers et al., Polyhydramnios and cerebellar atrophy: a prenatal presentation of mitochondrial encephalomyopathy caused by mutations in the FBXL4 gene. Clin Case Rep. 2016 Mar 16;4(4):425-8.

5. Sallevelt SCEH, de Koning B, Szklarczyk R et al,. A comprehensive strategy for exome-based preconception carrier screening. Genet Med. 2017 May;19(5):583-592.

6. Vissers LELM, van Nimwegen KJM, Schieving JH A clinical utility study of exome sequencing versus conventional genetic testing in pediatric neurology. Genet Med. 2017 Sep;19(9):1055-1063. 
Acknowledgements 

Being given the opportunity to do a $\mathrm{PhD}$ at Maastricht University was absolutely the best opportunity of my scientific career. It was a long journey and I felt lost now and then. Finally, I reached the finish line. I am very grateful to my family, my dear colleagues and friends from the Netherlands, Germany, Indonesia and Vietnam for your guidance, inspiration and support during all years of my PhD research.

I would like to express my first appreciation to my promotor, Bert, for giving me the opportunity to do my PhD on this interesting project. You have been a great mentor for me and I am fortunate to be part of your research group. Your guidance helped me in the most difficult times of research and writing this thesis. Without your support, consideration and motivation, my PhD would have been impossible. Your great advice and support on both research and on my future career have been invaluable.

Mike, my co-promotor, thank you so much for your support and supervision. I am amazed by your broad knowledge and skills. I learned from you critical thinking, data analysis and writing manuscripts. Your constructive and useful comments helped to make the project much easier for me. Many thanks again for your time, patience of reading my manuscripts, providing me with useful feedback and immense help.

I am very grateful to Rene, my co-promotor, for the excellent opportunity to improve my neurology knowledge. It was a pleasure to work with you and many thanks for your kind collaboration.

A special acknowledgement goes to Rosy. I know you to be warm-hearted, caring and thoughtful. You welcomed and helped me with all complicated paperwork on the first days I arrived in Maastricht. Thank you for arranging such a wonderful language course in Spa for me. I appreciate the cardigan which you so kindly knitted for me. Every time wearing it, I feel wrapped in love. It is a treasure to me now and forever.

I am thankful to you, Florence. You are incredible in many ways. I was continually amazed by your willingness to help me. You have been helpful in providing me with valuable advice many times on research and social life. I know I always can count on you. Your sincerity, consideration and dedication have inspired me. Everywhere I go, I wish to meet another Florence there. Thank you very much for all the favors you have done for me.

An, you have been so helpful when I first arrived in Maastricht. Spending Carnival time with you is one of the most enjoyable things I have done here. You talked with me what people rarely talked. That really opened my mind and made me feel a part of our group. I am truly grateful for your kindness.

Rita, you are my primary source for getting questions in general answered. I benefit from your great wisdom. You are a wonderful and generous colleague. It seemed that you understood my problems or needs and offered your assistance even before I asked you. I'll also not forget your help with furnishing my place. Many thanks for the time and help that you provided.

Jo Vanoevelen, thank you so much for your enthusiasm, willingness to share your expert knowledge on zebrafish with me and your invaluable help of constructive com- 
ments and suggestions throughout the experiments. I very much appreciate Radek of your ideas, helpful discussion and contributions to make my research productive. You are friendly, always call me when you see me on the roads. It was also very pleasurable talking with you.

My special thanks to Debby and Bianca, for providing me their significant contribution, cooperative, advice and guidance whenever I required. Debby, thank you so much for all your help and prompt replies to any of my questions and your enthusiasm for our project.

Rick, I know that I could always ask you for advice and opinions on lab related issues. You always give constructive suggestions and help with instrumentation and general lab questions. Besides, you are very knowledgeable about everything related to next generation sequencing. I very much appreciate your support and good advice for my future career.

My sincere appreciation will go to Bieke and Erika, my dear paranymphs, for organizing the reception for me! Bieke, talking with you after work always was a nice way to finish a day. You, a person with a big heart, have been a great, great help to me. Thank you so much for being available for all the times when I need you. I will never forget your efforts to contact people and drive me on rainy days for my benefits. I am touched by your concern about me. Erika, I consider you as the most friendly and cheerful person in our group. Whenever I needed something for my work, be it materials or advice, you were always there for me. Thank you so much.

My special thanks to Ivo. Although you spend a lot of time in zebrafish facility, you always brighten our room when you are there. You are an incredibly warm person who is passionate about helping other people. No wonder everyone likes you so much. Thank you very much for your insights, guidance, and assistance on zebrafish experiments. You are always extremely friendly, comforting me when I am down. I really appreciate every time you sat by me translating what people were talking, encouraging me to blend in. I will miss our "sitcoms", the time we walked together, or you rode me on my small bike.

I also thank Jo Beerens sincerely. You are very humorous and easy to talk to. You always provided immediate support for any computer problems I encountered. Many thanks for struggling with my computer and keep it alive.

Eveline and Wanwisa have been genuinely nice and extraordinary friendly to me. I want to especially thank Eveline for teaching me primary experiments in a gentle, supportive and enthusiastic way when I first got to Clinical Genetics. Wanwisa, I enjoyed every moment when I was with you. Thank you for your kind attention.

Furthermore, other past and present group members that I have had the pleasure to work with. I very much appreciated for a fruitful collaboration, sharing their research ideas, mutual help, nice conversations during social activities Iris, Bob, Auke, Romy, Tom, Ruby, Leo, Ellen, Barbie, Evelien, Janine, Markos, Michiel. Thank you for your companionship and help throughout my study. I also thank Els for being so helpful and friendly with 
administrative work. Miranda, Karen, Jieyi, Yilin, thank you for being incredibly kind and friendly to me.

Also outside the university, there are people that I would like to thank for making my stay in the Netherlands a pleasant and social one. My very sincere thanks to Gerry and Cyril for your hospitality, your parent-like treat, generous care and the home feeling whenever I was in need during my stay in Maastricht. Celebrating Christmas and New Year at your home is one of my most memorable festive time. I will miss you both very much!

I am grateful for friendships grown during my time at Maastricht which made my life more enjoyable.

Fitri, we have so much in common. I was lucky to find someone understands me like you. Thank you very much for the time with laughter, mutual encouragement, your care and love. Although you are now far away, one day we'll meet again and relive our beautiful days.

Christine, we moved to our student house at the same time. Love makes a house home and you are that love. Your welcome notes on my door, inspiration messages, big hugs, delicious cakes helped me a lot during difficult times. My gratitude also to you for your care, spiritual support and precious friendship during my stay at the Dorpstraat. I hope our friendship will continue to be strong in the future. And, I am looking forward to your visit me in Vietnam!

It is my pleasure to thank all my Vietnamese friends for all funny moments, unforgettable time we had together during many trips. Chit-chats with you brought peace to me during tough times. I am also thankful for your kind, emotional support.

Bố mẹ và gia đình yêu thương của con!

Vậy là con đã hoàn tất chặng đường vui nhưng cũng không ít khó khăn, dài hơn dự định. Trên hành trình đó, bố mẹ và gia đình luôn luôn bên cạnh con. Con cám ơn bố mẹ đã sinh ra con, nuôi nấng và dạy dỗ với tình yêu thương vô bờ bến để con có được như ngày hôm nay. Cuộc sống xa nhà với nhiều khó khăn bỡ ngỡ nhưng con vẫn vượt qua nhẹ nhàng. Bởi vì mỗi khi con gặp khó khăn nào, bố mẹ và gia đình luôn đưa ra những lời khuyên, đường hướng giải quyết tốt nhất, kịp thời tiếp thêm sức mạnh cho con. Con luôn cảm thấy may mắn được sống trong tình yêu thương ngập tràn của bố mẹ và gia đình. Con hiểu sâu sắc hơn những câu ca dao và câu thơ được học từ những ngày thơ ấu:

"Công cha như núi Thái Sơn

Nghĩa mẹ như nước trong nguồn chảy ra"

"Nước biển mênh mông không đong đầy tình mẹ

Mây trời lồng lộng không phủ kín công cha"

Con yêu và cám ơn bố mẹ, gia đình nhiều lắm. Con muốn kính dâng kết quả học tập của con như món quà tặng bố mẹ và gia đình. 
Lời cám ơn cuối cùng nhưng không kém phần quan trọng, cho phép tôi gửi lời cảm ơn chân thành nhất đến ban lãnh đạo và các đồng nghiệp tại Đại học Y Dược Tp. Hồ Chí Minh đã ủng hộ, giúp đỡ, tạo điều kiện để tôi hoàn thành tốt chương trình tiến sỹ tại Đại học Maastricht. 


\section{Curriculum Vitae}

Minh Nguyen was born on the 29th of December 1978, in Hanoi, Vietnam. In 2001, she received her Bachelor's degree in Biology at the University of Natural Sciences in HoChiMinh City, Vietnam. After graduation, she worked at the University of Medicine and Pharmacy in HoChiMinh city, Vietnam. She continued her studies at Chonnam National University, Korea in 2006 and graduated from Master in Molecular Medicine in 2008. On her return to Vietnam, she resumed her work at the University of Medicine and Pharmacy in HoChiMinh city, Vietnam. In September 2011, Minh started her PhD project under supervision of Prof. Dr. Smeets HJM, Dr. Gerards M, Dr. de Coo IFM at the department of Genetics and Cell Biology at Maastricht University, accomplishing this thesis. 



\section{List of publications}

Minh Nguyen, Iris Boesten, Debby M.E.I. Hellebrekers, Jo Vanoevelen, Rick Kamps, Bart de Koning, Irenaeus F.M. de Coo, Mike Gerards and Hubert J.M. Smeets. 'Pathogenic CWF19L1 variants as a novel cause of autosomal recessive cerebellar ataxia and atrophy.' Eur J Hum Genet. 2016 Apr;24(4):619-22.

Nguyen M, Boesten I, Hellebrekers DMEI, Mulder-den Hartog NM, de Coo IFM, Smeets HJM, Gerards M. 'Novel pathogenic SLC25A46 splice-site mutation causes an optic atrophy spectrum disorder.' Clin Genet. 2017 Jan;91(1):121-125.

Minh Nguyen, Irenaeus FM de Coo, Debby MEI Hellebrekers, Kees Schoonderwoerd, J. de Rijk- van Andel, Elvira Mulder, Rick Kamps, Bart de Koning, Mike Gerards and Hubert J M Smeets. 'Mutations in COX18 cause isolated complex IV deficiency, associated with neurodegeneration, chorea dystonic movement and type 1 diabetes.' Manuscript submitted.

Minh Nguyen, Jo Vanoevelen, Joost Nicolai, Ivo Eijkenboom, Rick Kamps, Constance T.R.M. Stumpel, Rinske F. Bos, Helene Pendeville, Hubert JM Smeets, Debby MEI Hellebrekers and Mike Gerards 'Whole exome sequencing reveals HKDC1 and MED2O as candidate genes in a child with a neurodegenerative disorder and congenital cataract.' Manuscript in preparation.

Tom E.J. Theunissen, Minh Nguyen, Rick Kamps, Alexandra T. Hendrickx, Suzanne C.E.H. Sallevelt, Ralph W.H. Gottschalk, Chantal M. Calis, Alphons P.M. Stassen, Bart de Koning, Elvira N. M. Mulder-Den Hartog, Kees Schoonderwoerd, Jo Vanoevelen, Radek Szklarczyk, Mike Gerards, Irenaeus F.M. de Coo, Debby M.E.I. Hellebrekers, Hubert J.M. Smeets 'Whole exome sequencing is the preferred, first strategy to identify known and novel causes of mitochondrial disease' Manuscript submitted. 
